OAK RIDGE
NATIONAL LABORATORY

MANAGED BY UT-BATTELLE

FOR THE DEPARTMENT OF ENERGY
ORNL/TM-2006/019

\section{Data Compilation for AGR-1 Baseline Coated Particle Composite LEU01-46T}

\author{
Revision 1
}

\author{
John D. Hunn and Richard A. Lowden
}

April 2006

This report was prepared as an account of work sponsored by an agency of the United States Government. Neither the United States Government nor any agency thereof, or any of their employees, makes any warranty, expressed or implied, or assumes any legal liability or responsibility for any third party's use, or the results of such use, of any information, apparatus, product or process disclosed in this report, or represents that its use by such third party would not infringe privately owned rights. 


\section{DOCUMENT AVAILABILITY}

Reports produced after January 1, 1996, are generally available free via the U.S. Department of Energy (DOE) Information Bridge.

Web site http://www.osti.gov/bridge

Reports produced before January 1, 1996, may be purchased by members of the public from the following source.

National Technical Information Service
5285 Port Royal Road
Springfield, VA 22161
Telephone 703-605-6000 (1-800-553-6847)
TDD 703-487-4639
Fax 703-605-6900
E-mail info @ntis.fedworld.gov
Web site http://www.ntis.gov/support/ordernowabout.htm

Reports are available to DOE employees, DOE contractors, Energy Technology Data Exchange (ETDE) representatives, and International Nuclear Information System (INIS)

representatives from the following source.

Office of Scientific and Technical Information

P.O. Box 62

Oak Ridge, TN 37831

Telephone 865-576-8401

Fax 865-576-5728

E-mail reports@adonis.osti.gov

Web site http://www.osti.gov/contact.html

This report was prepared as an account of work sponsored by an agency of the United States Government. Neither the United States Government nor any agency thereof, nor any of their employees, makes any warranty, express or implied, or assumes any legal liability or responsibility for the accuracy, completeness, or usefulness of any information, apparatus, product, or process disclosed, or represents that its use would not infringe privately owned rights. Reference herein to any specific commercial product, process, or service by trade name, trademark, manufacturer, or otherwise, does not necessarily constitute or imply its endorsement, recommendation, or favoring by the United States Government or any agency thereof. The views and opinions of authors expressed herein do not necessarily state or reflect those of the United States Government or any agency thereof. 


\title{
Data Compilation for AGR-1 Baseline Coated Particle Composite LEU01-46T
}

\author{
John D. Hunn and Richard A. Lowden \\ Oak Ridge National Laboratory
}

This document is a compilation of characterization data for the AGR-1 baseline coated particle composite LEU01-46T, a composite of four batches of TRISO-coated $350 \mu \mathrm{m} \mathrm{19.7 \%} \mathrm{low}$ enrichment uranium oxide/uranium carbide kernels (LEUCO). The AGR-1 TRISO-coated particles consist of a spherical kernel coated with a 50\% dense carbon buffer layer (100 $\mu \mathrm{m}$ nominal thickness) followed by a dense inner pyrocarbon layer (40 $\mu \mathrm{m}$ nominal thickness) followed by a $\mathrm{SiC}$ layer ( $35 \mu \mathrm{m}$ nominal thickness) followed by another dense outer pyrocarbon layer (40 $\mu \mathrm{m}$ nominal thickness). The coated particles were produced by ORNL for the Advanced Gas Reactor Fuel Development and Qualification (AGR) program to be put into compacts for insertion in the first irradiation test capsule, AGR-1. The kernels were obtained from BWXT and identified as composite G73D-20-69302. The BWXT kernel lot G73D-2069302 was riffled into sublots for characterization and coating by ORNL and identified as LEU01-\#\# (where \#\# is a series of integers beginning with 01).

Additional particle batches were coated with only buffer or buffer plus inner pyrocarbon (IPyC) layers using similar process conditions as used for the full TRISO batches comprising the LEU01-46T composite. These batches were fabricated in order to qualify that the process conditions used for buffer and IPyC would produce acceptable densities, as described in sections 8 and 9. These qualifying batches used $350 \mu \mathrm{m}$ natural uranium oxide/uranium carbide kernels (NUCO). The kernels were obtained from BWXT and identified as composite G73B-NU-69300. The use of NUCO surrogate kernels is not expected to significantly effect the densities of the buffer and IPyC coatings. Confirmatory batches using LEUCO kernels from G73D-20-69302 were coated and characterized to verify this assumption.

The AGR-1 Fuel Product Specification and Characterization Guidance (INL EDF-4380, Rev. 6) provides the requirements necessary for acceptance of the fuel manufactured for the AGR-1 irradiation test. Sections 5.2 and 5.3 of EDF-4380 provide the property requirements for the coated particle batches and coated particle composite. The Statistical Sampling Plan for AGR Fuel Materials (INL EDF-4542, Rev. 6) provides additional guidance regarding statistical methods for product acceptance and recommended sample sizes. The procedures for characterizing and qualifying the particles are outlined in ORNL product inspection plans: AGRCHAR-PIP-01, AGR-CHAR-PIP-02, AGR-CHAR-PIP-03, and AGR-CHAR-PIP-04. The inspection report forms generated by these product inspection plans document the product acceptance for the property requirements listed in sections 5.2 and 5.3 of EDF-4380.

Note that, as the characterization procedures evolved and improved during the characterization of this composite, several important properties were remeasured (e.g., average kernel weight, buffer density, and coating thicknesses for the particle batches). This is discussed in sections 6-8 and sections 11-14, and the superceded data is included in section 16. 


\section{$\underline{\text { Table of Contents }}$}

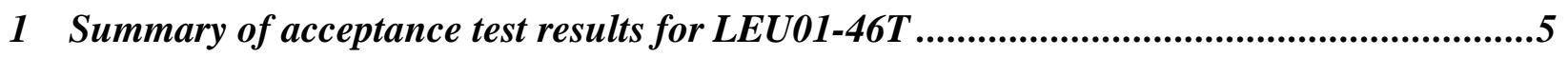

2 Product ID's associated with LEU01-46T ................................................................15

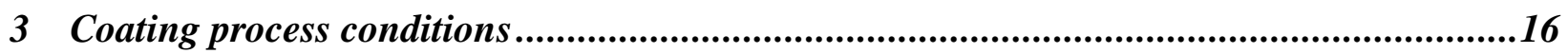

4 Classification of coated particles ..............................................................................32

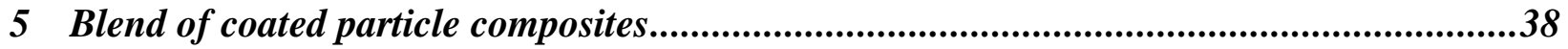

6 Characterization of LEUCO kernel composite .........................................................39

7 Characterization of NUCO kernel composite ..........................................................46

8 Characterization of buffer on interrupted batches ........................................................53

9 Characterization of IPyC on interrupted batches .........................................................67

10 Characterization of IPyC on additional interrupted batches .......................................91

11 Characterization of first batch of TRISO-coated particles ...........................................106

12 Characterization of second batch of TRISO-coated particles ....................................121

13 Characterization of third batch of TRISO-coated particles ........................................136

14 Characterization of fourth batch of TRISO-coated particles.....................................151

15 Characterization of TRISO-coated particle composite .............................................166

16 Superceded data ..........................................................................................205 


\section{Summary of acceptance test results for LEU01-46T}

This section contains inspection report forms (IRF's) associated with the coated particle composite LEU01-46T. These inspection report forms summarize the acceptance testing performed according to the product inspection plans: AGR-CHAR-PIP-02, AGR-CHAR-PIP-03, and AGR-CHAR-PIP-04. The information in these forms covers all the property specifications listed in sections 5.2 and 5.3 of the AGR-1 Fuel Product Specification and Characterization Guidance document INL EDF-4380, Rev. 6. The coated particle composite, LEU01-46T, was found to meet all the requirements in these two sections of EDF-4380, Rev. 6.

These inspection report forms also appear in later sections of this compilation, accompanied by the associated data report forms showing the results of each individual measurement.

Table 1-1 is provided for quick reference. It gives the mean values of key variable properties of the coated particle composite, LEU01-46T. For standard deviations of the distribution of the measured values see the appropriate IRF. For discussions on the uncertainty in these values, see the associated data acquisition methods and data report forms. The kernel diameter and density values are from ORNL measurements made for information only. The buffer and IPyC densities in the table are averages of the means for the individual interrupted batches (including confirmation batches on LEUCO), rather than direct measurements on the composite. The OPyC density in the table is an average of the mean OPyC density for each batch weighted by the fraction of each batch in the composite.

Table 1-1: Quick reference table for key variable properties of LEU01-46T.

\begin{tabular}{|l|c|}
\hline \multicolumn{1}{|c|}{ Property } & Mean \\
\hline Average kernel diameter $(\mu \mathrm{m})$ & 349.7 \\
\hline Kernel envelope density $\left(\mathrm{Mg} / \mathrm{m}^{3}\right)$ & 10.924 \\
\hline Average buffer thickness $(\mu \mathrm{m})$ & 103.5 \\
\hline Average IPyC thickness $(\mu \mathrm{m})$ & 39.4 \\
\hline Average SiC thickness $(\mu \mathrm{m})$ & 35.3 \\
\hline Average OPyC thickness $(\mu \mathrm{m})$ & 41.0 \\
\hline Buffer envelope density $\left(\mathrm{Mg} / \mathrm{m}^{3}\right)$ (interrupted batches) & 1.10 \\
\hline IPyC sink/float density $\left(\mathrm{Mg} / \mathrm{m}^{3}\right)$ (interrupted batches) & 1.904 \\
\hline SiC sink/float density $\left(\mathrm{Mg} / \mathrm{m}^{3}\right)$ & 3.2075 \\
\hline OPyC sink/float density $\left(\mathrm{Mg} / \mathrm{m}^{3}\right)($ weighted average) & 1.907 \\
\hline IPyC anisotropy $($ BAFo equivalent) & 1.022 \\
\hline OPyC anisotropy $(B A F o ~ e q u i v a l e n t)$ & 1.019 \\
\hline
\end{tabular}


Table 1-2 is also provided for quick reference. It gives the upper limit of the $95 \%$ confidence interval of the defect fraction for key attribute properties of the coated particle composite, LEU01-46T. In other words, these values are the lowest tolerance limits for which the composite would be deemed acceptable at $95 \%$ confidence based on the particular sample that was measured. For the actual number of trials and number of failures observed, see the inspection report form for the coated particle composite.

Table 1-2: Quick reference table for key attribute properties of LEU01-46T.

\begin{tabular}{|l|c|}
\hline \multicolumn{1}{|c|}{ Property } & Defect Fraction \\
\hline Particles with SiC gold spot defects & $\leq 1.0 \times 10^{-3}$ \\
\hline Particle aspect ratio & $\leq 3.9 \times 10^{-3}$ \\
\hline Particles with burn-leach defects & $\leq 2.5 \times 10^{-5}$ \\
\hline Particles with missing OPyC & $\leq 9.6 \times 10^{-5}$ \\
\hline
\end{tabular}




\begin{tabular}{|c|c|c|c|c|c|c|c|c|c|}
\hline Procedure: & \multicolumn{9}{|c|}{ AGR-CHAR-PIP-02 Rev. 4} \\
\hline Batch 1 ID: & \multicolumn{9}{|c|}{ NUCO $350-25 B$} \\
\hline Batch 1 description: & \multicolumn{9}{|c|}{ Buffer-coated BWXT kernel composite 69300} \\
\hline Batch 2 ID: & \multicolumn{9}{|c|}{ NUCO $350-368$} \\
\hline Batch 2 description: & \multicolumn{9}{|c|}{ Buffer-coated BWXT kernel composite 69300} \\
\hline Batch 3 ID: & \multicolumn{9}{|c|}{ NUCO350-54B } \\
\hline Batch 3 description: & \multicolumn{9}{|c|}{ Buffer-coated BWXT kernel composite 69300} \\
\hline \multirow[b]{2}{*}{ Property } & \multicolumn{4}{|c|}{ Measured Data } & Specification & \multirow[b]{2}{*}{ Acceptance Criteria } & \multirow{2}{*}{$\begin{array}{l}\text { Acceptance } \\
\text { Test Value }\end{array}$} & \multirow{2}{*}{$\begin{array}{c}\text { Pass } \\
\text { or } \\
\text { fail }\end{array}$} & \multirow{2}{*}{$\begin{array}{c}\text { Data } \\
\text { Records }\end{array}$} \\
\hline & $\begin{array}{c}\text { Mean } \\
(x)\end{array}$ & $\begin{array}{c}\text { Std. Dev. } \\
\text { (s) }\end{array}$ & $\begin{array}{c}\text { \# measured } \\
\text { (n) }\end{array}$ & $\begin{array}{c}t \text { value } \\
\text { (t) }\end{array}$ & $\begin{array}{c}\text { INL EDF }-4380 \\
\text { Rev. } 6\end{array}$ & & & & \\
\hline \multirow{2}{*}{ Batch 1: Buffer envelope density $\left(\mathrm{Mg} / \mathrm{m}^{3}\right)$} & \multirow{2}{*}{1.08} & \multirow{2}{*}{0.04} & \multirow{2}{*}{5} & \multirow{2}{*}{2.132} & \multirow{2}{*}{$\begin{array}{c}\text { mean } \\
1.03 \pm 0.15 \\
\end{array}$} & $A=x-t s / \sqrt{ } n \geq 0.88$ & 1.04 & pass & \multirow{2}{*}{$\begin{array}{l}\text { DRF-16 } \\
\text { DRF-22 }\end{array}$} \\
\hline & & & & & & $B=x+t s / \sqrt{n} \leq 1.18$ & 1.12 & pass & \\
\hline \multirow{2}{*}{ Batch 2: Buffer envelope density $\left(\mathrm{Mg} / \mathrm{m}^{3}\right)$} & \multirow{2}{*}{1.11} & \multirow[t]{2}{*}{0.05} & \multirow[t]{2}{*}{5} & \multirow[t]{2}{*}{2.132} & \multirow{2}{*}{$\begin{array}{c}\text { mean } \\
1.03 \neq 0.15\end{array}$} & $A=x-t s / \sqrt{n} \geq 0.88$ & 1.06 & pass & \multirow{2}{*}{$\begin{array}{l}\text { DRF-16 } \\
\text { DRF-22 }\end{array}$} \\
\hline & & & & & & $B=x+t s / \sqrt{n} \leq 1.18$ & 1.16 & pass & \\
\hline \multirow{2}{*}{ Batch 3: Buffer envelope density $\left(\mathrm{Mg} / \mathrm{m}^{3}\right)$} & \multirow[t]{2}{*}{1.11} & \multirow[t]{2}{*}{0.04} & \multirow[t]{2}{*}{5} & \multirow[t]{2}{*}{2.132} & \multirow{2}{*}{$\begin{array}{c}\text { mean } \\
1.03 \pm 0.15\end{array}$} & $A=x-t s / \sqrt{n} \geq 0.88$ & 1.07 & pass & \multirow{2}{*}{$\begin{array}{l}\text { DRF-16 } \\
\text { DRF-22 }\end{array}$} \\
\hline & & & & & & $B=x+t s / \sqrt{n} \leq 1.18$ & 1.15 & pass & \\
\hline
\end{tabular}

\section{Comments}

Standard deviations are $\sqrt{5}$ times the uncertainties in buffer density (standard errors) reported on DRF-16.

Average thickness of buffer was $108 \mu \mathrm{m}$ based on average envelope volume of $9.25 \mathrm{E}-5 \mathrm{cc}$ (effective diameter of $561 \mu \mathrm{m}$ ) and average kernel diameter of $345 \mu \mathrm{m}$.

Average thickness of buffer was $108 \mu \mathrm{m}$ based on average outer diameter of $561 \mu \mathrm{m}$ obtained per DAM-10 and average kernel diameter of $345 \mu \mathrm{m}$.

Confirmatory batch on LEUCO kernels, LEU01-16E: mean buffer density $=1.10 \mathrm{~g} / \mathrm{cc}$.

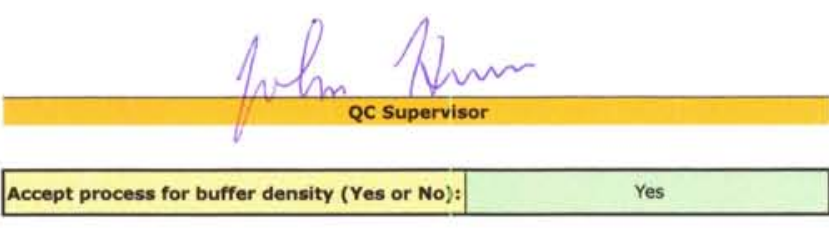

QA Revieiver
$3-10-06$ Date 


\section{Inspection Report form IRF-02B: Interrupted Coating Batches - IPvC Density}

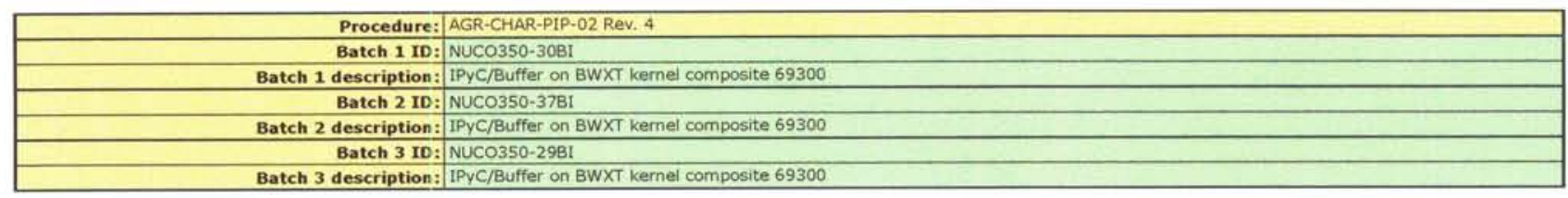

\begin{tabular}{|c|c|c|c|c|c|c|c|c|c|}
\hline \multirow[b]{2}{*}{ Property } & \multicolumn{4}{|c|}{ Measured Data } & \multirow{2}{*}{$\begin{array}{c}\text { Specification } \\
\text { INL. EDF- } 4380 \\
\text { Rev, } 6\end{array}$} & \multirow[b]{2}{*}{ Acceptance Criteria } & \multirow{2}{*}{$\begin{array}{l}\text { Acceptance } \\
\text { Test Value }\end{array}$} & \multirow{2}{*}{$\begin{array}{l}\text { Pass } \\
\text { or } \\
\text { fail }\end{array}$} & \multirow{2}{*}{$\begin{array}{c}\text { Data } \\
\text { Records }\end{array}$} \\
\hline & $\begin{array}{l}\text { Mean } \\
(x)\end{array}$ & \begin{tabular}{|c|} 
Std. Dev. \\
(s)
\end{tabular} & $\begin{array}{c}* \text { measured } \\
(\mathrm{n})\end{array}$ & $\begin{array}{l}k \text { or } t \\
\text { value }\end{array}$ & & & & & \\
\hline \multirow{4}{*}{ Batch 1: IPyC sink/float density $\left(\mathrm{Mg} / \mathrm{m}^{3}\right)$} & \multirow{4}{*}{1.8917} & \multirow{4}{*}{0.0113} & \multirow{4}{*}{25} & \multirow{2}{*}{1,711} & \multirow{2}{*}{$\begin{array}{c}\text { mean } \\
1.90 \pm 0.05\end{array}$} & $A=x-t s / \sqrt{ } n \geq 1.85$ & 1.888 & pass & \multirow{4}{*}{ DRF-03 } \\
\hline & & & & & & $\mathrm{B}=\mathrm{x}+\mathrm{ts} / \sqrt{ } \mathrm{n} \leq 1.95$ & 1.896 & pass & \\
\hline & & & & \multirow{2}{*}{3.158} & \multirow{2}{*}{$\begin{array}{c}\text { dispersion } \\
\leq 0.01 \leq 1.80 \\
\leq 0.01 \geq 2.00 \\
\end{array}$} & $C=x-k s>1.80$ & 1.856 & pass & \\
\hline & & & & & & $D=x+k s<2.00$ & 1.927 & pass & \\
\hline \multirow{3}{*}{ Batch 2: IPyC sink/float density $\left(\mathrm{Mg} / \mathrm{m}^{3}\right)$} & \multirow{3}{*}{1.9038} & \multirow{3}{*}{0.0153} & \multirow{3}{*}{21} & 1.725 & $\begin{array}{c}\text { mean } \\
1.90 \pm 0.05\end{array}$ & $A=x-t s / \sqrt{ } n \geq 1.85$ & 1.898 & pass & \multirow{3}{*}{ DRF-03 } \\
\hline & & & & \multirow{2}{*}{3.262} & \multirow{2}{*}{$\begin{array}{c}\text { dispersion } \\
\leq 0.01 \leq 1.80 \\
\leq 0.01 \geq 2.00 \\
\end{array}$} & $C=x-k s>1.80$ & 1.854 & pass & \\
\hline & & & & & & $D=x+k s<2.00$ & 1.954 & pass & \\
\hline \multirow{4}{*}{ Batch 3: IPyC sink/float density $\left(\mathrm{Mg} / \mathrm{m}^{3}\right)$} & \multirow{4}{*}{1.9112} & \multirow{4}{*}{0.0142} & \multirow{4}{*}{20} & \multirow{2}{*}{1.729} & \multirow{2}{*}{$\begin{array}{c}\text { mean } \\
1.90 \pm 0.05\end{array}$} & $A=x-t s / \sqrt{ } n \geq 1.85$ & 1.906 & pass & \multirow{4}{*}{ DRF-03 } \\
\hline & & & & & & $B=x+t s / V n \leq 1.95$ & 1.917 & pass & \\
\hline & & & & \multirow{2}{*}{3.295} & \multirow{2}{*}{$\begin{array}{c}\text { dispersion } \\
\leq 0.01 \leq 1.80 \\
\leq 0.01 \geq 2.00\end{array}$} & $C=x-k s>1.80$ & 1.864 & pass & \\
\hline & & & & & & $D=x+k s<2.00$ & 1.958 & pass & \\
\hline
\end{tabular}

\section{Comments}

$95 \%$ confidence interval for Buffer thickness in composite $=(104 \mu \mathrm{m}, 106 \mu \mathrm{m})$ with $<1 \% \leq 55 \mu \mathrm{m}$.

$95 \%$ confidence interval for IPyC thickness in composite $=(34.2 \mu \mathrm{m}, 34.9 \mu \mathrm{m})$ with $>1 \% \leq 30 \mu \mathrm{m}$ and $<1 \% \geq 56 \mu \mathrm{m}$.

Confirmatory batch on LEUCO kernels, LFU01-15I: mean IPyC density $=1.9074 \mathrm{~g} / \mathrm{cc}$.

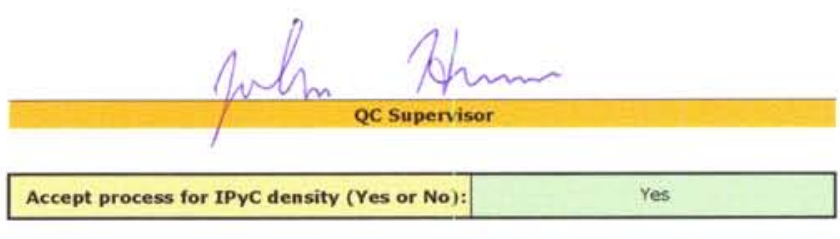

$$
3-10-06
$$

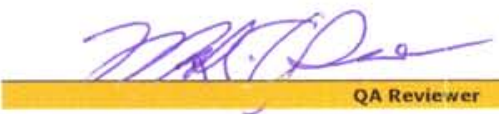

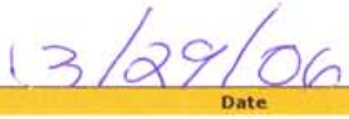


ORNL/TM-2006/019, Rev. 1

Inspection Report Form IRF-03: Coated Particle Batches

Procedure: AGR-CHAR-PIP-03 Rev. 2

Coated particle batch ID: LEU01-21T

Coated particle batch description: TRISO on BWXT kernel composite 69302

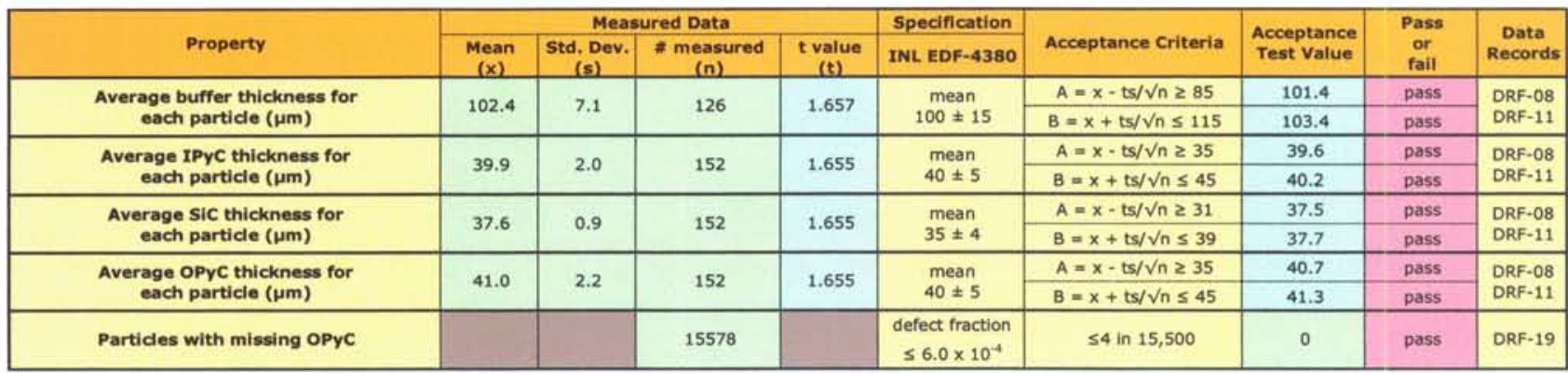

Comments

Goldspot analysis performed on missing OPyC sample for information only. 10 out of 15578 gold spots observed.

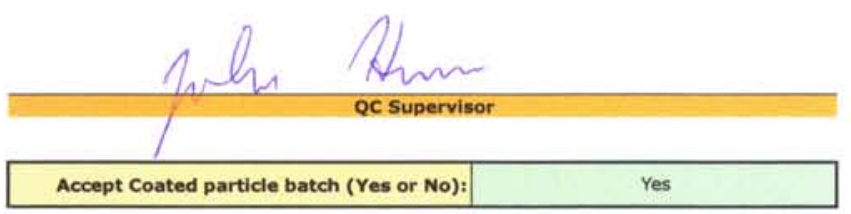

Dareviewer
$3-23-06$

Date

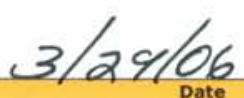


ORNL/TM-2006/019, Rev. 1

Inspection Report Form IRF-03: Coated Particle Batches

Procedure: AGR-CHAR-PIP-03 Rev. 2

Coated particle batch ID: LEUO1-35T

Coated particle batch description: TRISO on BWXT kernel composite 69302

\begin{tabular}{|c|c|c|c|c|c|c|c|c|c|}
\hline \multirow[b]{2}{*}{ Property } & \multicolumn{4}{|c|}{ Measured Data } & Specification & \multirow[b]{2}{*}{ Acceptance Criteria } & \multirow{2}{*}{$\begin{array}{l}\text { Acceptance } \\
\text { Test Value }\end{array}$} & \multirow{2}{*}{$\begin{array}{l}\text { Pass } \\
\text { or } \\
\text { fail }\end{array}$} & \multirow{2}{*}{$\begin{array}{c}\text { Data } \\
\text { Records }\end{array}$} \\
\hline & $\begin{array}{c}\text { Mean } \\
(x)\end{array}$ & $\begin{array}{c}\text { Std. Dev. } \\
(\mathrm{s})\end{array}$ & $\begin{array}{l}\text { \# measured } \\
\text { (n) }\end{array}$ & $\begin{array}{c}t \text { value } \\
\text { (t) }\end{array}$ & INL EDF-4380 & & & & \\
\hline \multirow{2}{*}{$\begin{array}{l}\text { Average buffer thickness for } \\
\text { each particle }(\mu \mathrm{m})\end{array}$} & \multirow{2}{*}{103.4} & \multirow{2}{*}{8.2} & \multirow{2}{*}{163} & \multirow{2}{*}{1.654} & \multirow{2}{*}{$\begin{array}{c}\text { mean } \\
100 \pm 15\end{array}$} & $A=x-t s / \sqrt{n} \geq 85$ & 102.3 & pass & \multirow{2}{*}{$\begin{array}{l}\text { DRF-08 } \\
\text { DRF-11 }\end{array}$} \\
\hline & & & & & & $B=x+t s / \sqrt{n} \leq 115$ & 104.5 & pass & \\
\hline \multirow{2}{*}{$\begin{array}{l}\text { Average IPyC thickness for } \\
\text { each particle }(\mu \mathrm{m})\end{array}$} & \multirow{2}{*}{39.7} & \multirow{2}{*}{2.2} & \multirow{2}{*}{214} & \multirow{2}{*}{1.652} & \multirow{2}{*}{$\begin{array}{l}\text { mean } \\
40 \pm 5\end{array}$} & $A=x-t s / \sqrt{n} \geq 35$ & 39.5 & pass & \multirow{2}{*}{$\begin{array}{l}\text { DRF-08 } \\
\text { DRF-11 }\end{array}$} \\
\hline & & & & & & $B=x+t s / \sqrt{n} \leq 45$ & 39.9 & pass & \\
\hline \multirow{2}{*}{$\begin{array}{l}\text { Average SiC thickness for } \\
\text { each particle }(\mu \mathrm{m})\end{array}$} & \multirow{2}{*}{36.3} & \multirow{2}{*}{1.0} & \multirow{2}{*}{214} & \multirow{2}{*}{1.652} & \multirow{2}{*}{$\begin{array}{l}\text { mean } \\
35 \pm 4\end{array}$} & $A=x-t s / \sqrt{n} \geq 31$ & 36.2 & pass & \multirow{2}{*}{$\begin{array}{l}\text { DRF-08 } \\
\text { DRF-11 }\end{array}$} \\
\hline & & & & & & $B=x+t s / \sqrt{n} \leq 39$ & 36.4 & pass & \\
\hline $\begin{array}{l}\text { Average OPyC thickness for } \\
\text { each particle }(\mu \mathrm{m})\end{array}$ & 40.2 & 1.9 & 214 & 1.652 & $\begin{array}{l}\text { mean } \\
40 \pm 5\end{array}$ & $\mathrm{~B}=\mathrm{x}+\mathrm{ts} / \sqrt{ } \mathrm{n} \leq 45$ & 40.4 & pass & $\begin{array}{l}\text { DRF-08 } \\
\text { DRF-11 }\end{array}$ \\
\hline Particles with missing OPyC & & & 15622 & & $\begin{array}{l}\text { defect fraction } \\
\leq 6.0 \times 10^{-4}\end{array}$ & $\leq 4$ in 15,500 & 0 & pass & DRF-19 \\
\hline
\end{tabular}

\section{Comments}

Goldspot analysis performed on missing OPyC sample for information only. 17 out of 15622 gold spots observed.

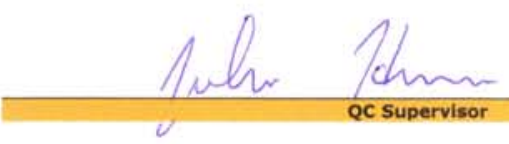

Accept Coated particle batch (Yes or No):

Yes

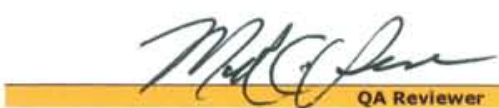

$3-23-06$

Date 
ORNL/TM-2006/019, Rev. 1

\section{Inspection Report Form IRF-03: Coated Particle Batches}

Procedure: AGR-CHAR-PIP-03 Rev. 2

Coated particle batch ID: LEU01-27T

Coated particle batch description: TRISO on BWXT kernel composite 69302

\begin{tabular}{|c|c|c|c|c|c|c|c|c|c|}
\hline \multirow[b]{2}{*}{ Property } & \multicolumn{4}{|c|}{ Measured Data } & \multirow{2}{*}{$\begin{array}{l}\text { Specification } \\
\text { INL EDF-4380 }\end{array}$} & \multirow[b]{2}{*}{ Acceptance Criteria } & \multirow{2}{*}{$\begin{array}{l}\text { Acceptance } \\
\text { Test Value }\end{array}$} & \multirow{2}{*}{$\begin{array}{l}\text { Pass } \\
\text { or } \\
\text { fail } \\
\end{array}$} & \multirow{2}{*}{$\begin{array}{l}\text { Data } \\
\text { Records }\end{array}$} \\
\hline & $\begin{array}{c}\text { Mean } \\
(x)\end{array}$ & $\begin{array}{c}\text { Std. Dev. } \\
\text { (s) }\end{array}$ & $\begin{array}{c}\text { \# measured } \\
\text { (n) }\end{array}$ & $\begin{array}{l}t \text { value } \\
\text { (t) }\end{array}$ & & & & & \\
\hline \multirow{2}{*}{$\begin{array}{l}\text { Average buffer thickness for } \\
\text { each particle }(\mu \mathrm{m})\end{array}$} & \multirow{2}{*}{104.9} & \multirow{2}{*}{8.2} & \multirow{2}{*}{188} & \multirow{2}{*}{1.653} & \multirow{2}{*}{$\begin{array}{c}\text { mean } \\
100 \pm 15\end{array}$} & $A=x-t s / \sqrt{n} \geq 85$ & 103.9 & pass & \multirow{2}{*}{$\begin{array}{l}\text { DRF-08 } \\
\text { DRF-11 }\end{array}$} \\
\hline & & & & & & $B=x+t s / \sqrt{n} \leq 115$ & 105.9 & pass & \\
\hline \multirow{2}{*}{$\begin{array}{l}\text { Average IPyC thickness for } \\
\text { each particle }(\mu \mathrm{m})\end{array}$} & \multirow{2}{*}{39.0} & \multirow{2}{*}{2.2} & \multirow{2}{*}{249} & \multirow{2}{*}{1.651} & \multirow{2}{*}{$\begin{array}{l}\text { mean } \\
40 \pm 5\end{array}$} & $A=x-t s / \sqrt{n} \geq 35$ & 38.8 & pass & \multirow{2}{*}{$\begin{array}{l}\text { DRF-08 } \\
\text { DRF-11 }\end{array}$} \\
\hline & & & & & & $B=x+t s / \sqrt{n} \leq 45$ & 39.2 & pass & \\
\hline \multirow{2}{*}{$\begin{array}{l}\text { Average SiC thickness for } \\
\text { each particle }(\mu \mathrm{m})\end{array}$} & \multirow{2}{*}{34.6} & \multirow{2}{*}{1.2} & \multirow{2}{*}{248} & \multirow{2}{*}{1.651} & \multirow{2}{*}{$\begin{array}{l}\text { mean } \\
35 \pm 4\end{array}$} & $A=x-t s / \sqrt{n} \geq 31$ & 34.5 & pass & \multirow{2}{*}{$\begin{array}{l}\text { DRF-08 } \\
\text { DRF-11 }\end{array}$} \\
\hline & & & & & & $B=x+t s / \sqrt{n} \leq 39$ & 34.7 & pass & \\
\hline $\begin{array}{l}\text { Average OPyC thickness for } \\
\text { each particle }(\mu \mathrm{m})\end{array}$ & 41.7 & 2.0 & 248 & 1.651 & $\begin{array}{l}\text { mean } \\
40 \pm 5\end{array}$ & $B=x+t s / \sqrt{n} \leq 45$ & 41.9 & pass & $\begin{array}{l}\text { DRF-08 } \\
\text { DRF-11 }\end{array}$ \\
\hline Particles with missing OPyC & & & 15680 & & $\begin{array}{l}\text { defect fraction } \\
\leq 6.0 \times 10^{-4}\end{array}$ & $\leq 4$ in 15,500 & 0 & pass & DRF-19 \\
\hline
\end{tabular}

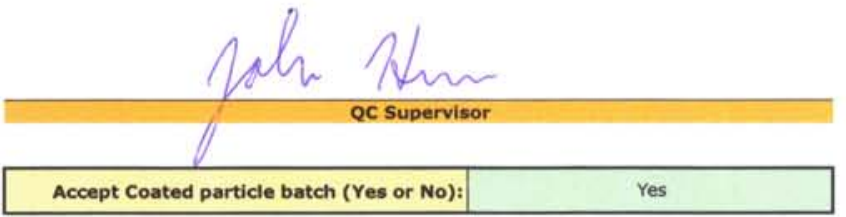

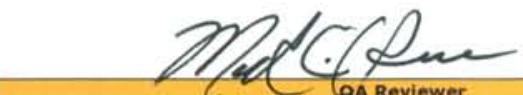

$3-23-06$

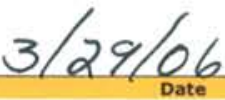


ORNL/TM-2006/019, Rev. 1

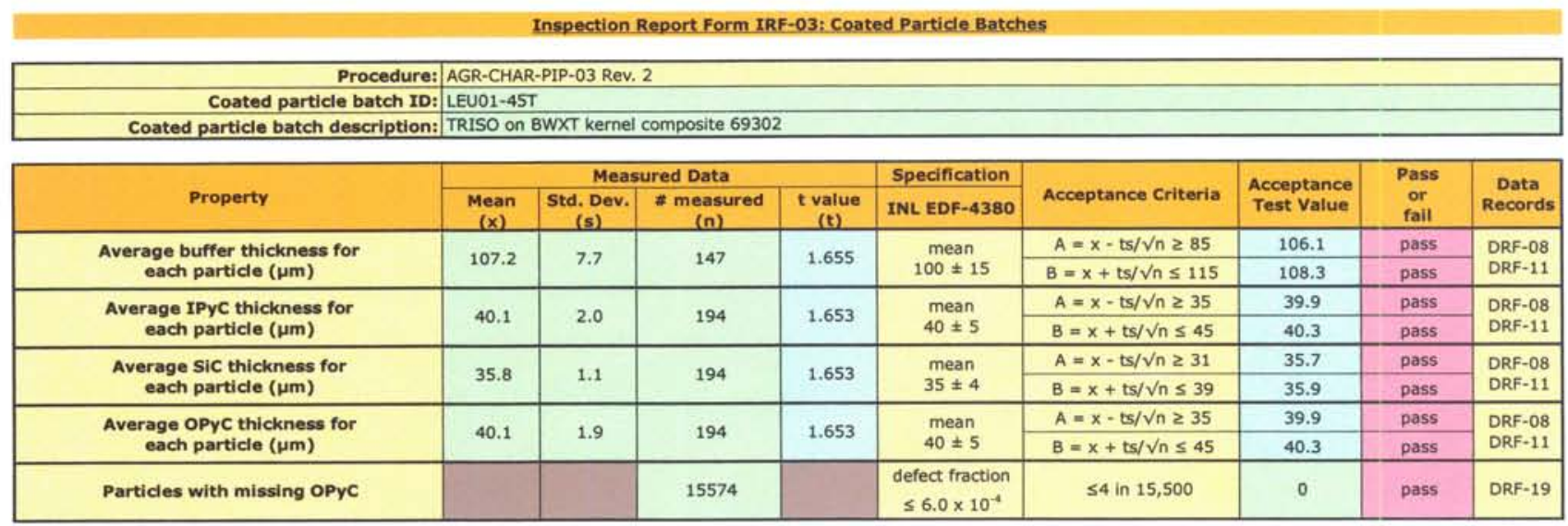

\begin{aligned} Goldspot analysis performed on missing OPYC sample for information only. 3 out of 15574 gold spots \\ \hline\end{aligned}

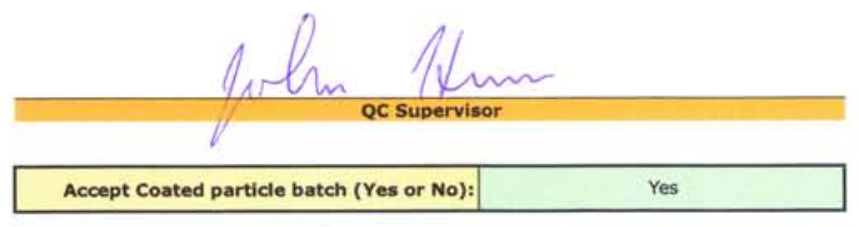

$3-23-06$

arectioner

$3 / 29 / 06$

12 


\section{Inspection Report form IRF-04A: Coated Particle Composites}

\begin{tabular}{|r|rl}
\hline & Procedure: & AGR-CHAR-PIP-04 Rev. 2 \\
\hline
\end{tabular}

$\begin{aligned} \text { Procedure: } & \text { AGR-CHAR-PIP-C } \\ \text { Coated particle composite ID: } & \text { LEU01-46T }\end{aligned}$

Coated particle composite description: Baseline Composite: TRISO on BWXT kernel composite 69302

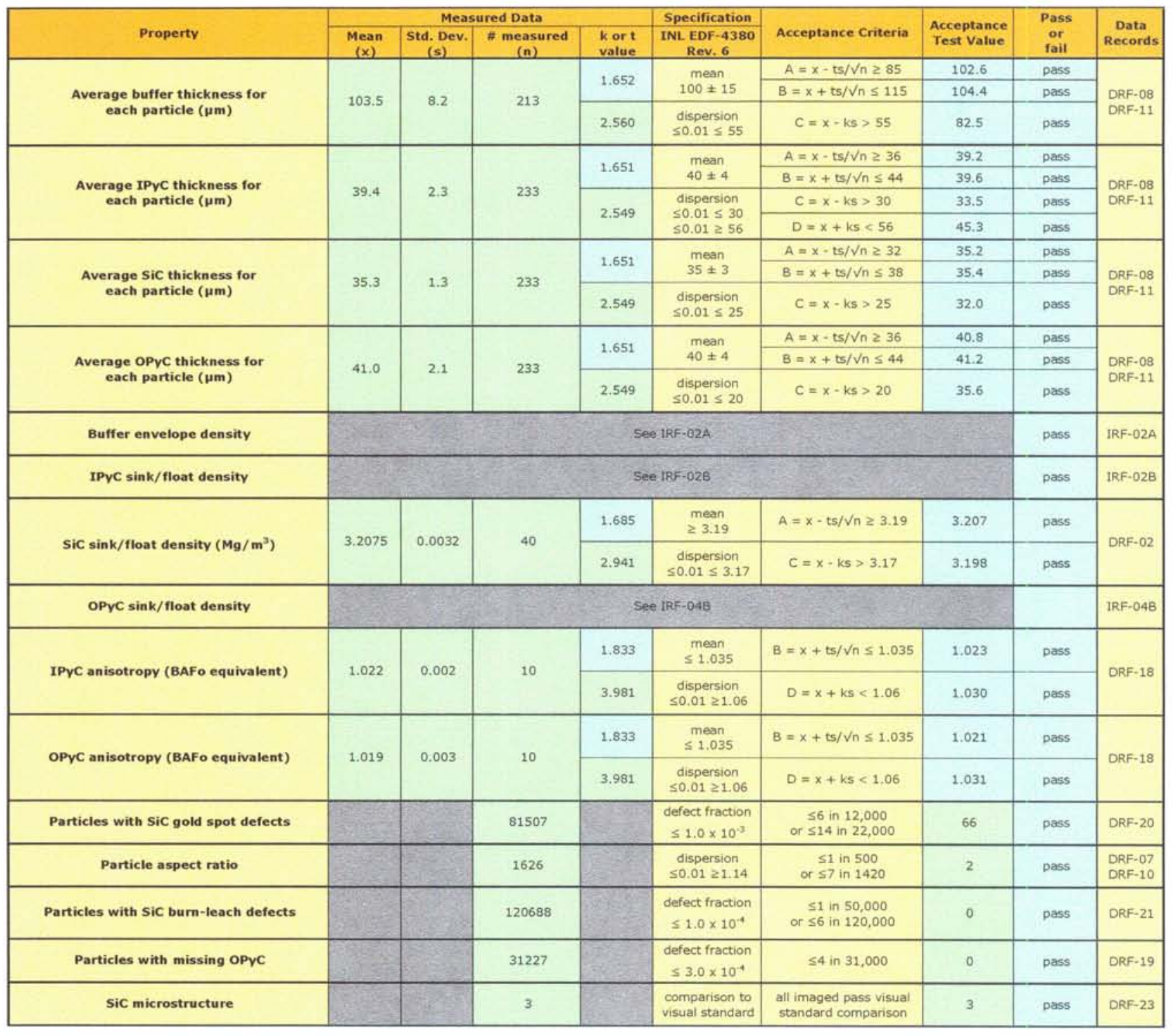

Comments

66 out of 81507 gold spot defects passes the acceptance criterion of $\leq 66$ in 81100 indicating $51 \mathrm{E}-3$ defects with $95 \%$ confidence.

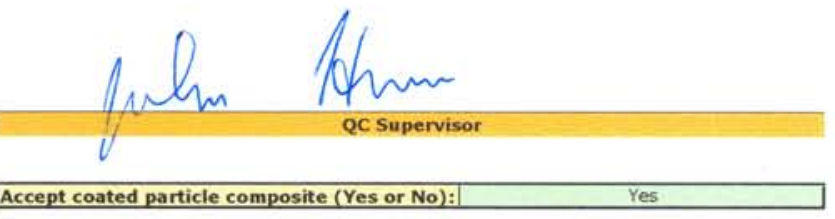

$$
3-21-06
$$


ORNL/TM-2006/019, Rev. 1

Inspection Report Form IRF-04B: Outer Pyrocarbon Density

\section{Inlm $1 / 4$ min \\ QC Supervisor}

\section{$3-21-06$}

\section{Accept composite for OPVC density (Yes or No):}

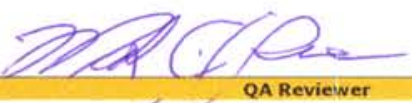

Yes

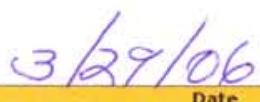




\section{Product ID's associated with LEU01-46T}

$\underline{\text { Kernels }}$

LEU01-\#\# (from BWXT G73D-20-69302)

NUCO350-\#\# (from BWXT G73B-NU-69300)

Buffer-only particles

NUCO350-25B

NUCO350-36B

NUCO350-54B

NUCO350-58B (composite of $25 \mathrm{~B}+36 \mathrm{~B}+54 \mathrm{~B}$, for information only)

LEU01-16B (confirmation on LEUCO kernels, for information only)

$\underline{\text { IPyC/Buffer-only particles }}$

NUCO350-30BI

NUCO350-37BI

NUCO350-29BI

NUCO350-66BI (composite of 30BI+37BI+29BI, for information only)

LEU01-15I (confirmation on LEUCO kernels, for information only)

LEU01-23I (confirmation on LEUCO kernels, for information only)

LEU01-26I (confirmation on LEUCO kernels, for information only)

LEU01-41I (confirmation on LEUCO kernels, for information only)

$\underline{\text { TRISO-coated particles }}$

LEU01-21T

LEU01-35T

LEU01-27T

LEU01-45T

LEU01-46T (composite of 21T+35T+27T+45T) 


\section{Coating process conditions}

The following pages contain coating process conditions for all coated particle batches associated with the LEU01-46T coated particle composite. These particles were coated within the baseline process conditions listed in section 3.1 of the AGR-1 Fuel Product Specification and Characterization Guidance document INL EDF-4380, Rev. 6.

$\underline{\text { Buffer-only interrupted batches }}$

NUCO350-25B

NUCO350-36B

NUCO350-54B

LEU01-16B

$\underline{\text { IPyC/Buffer-only interrupted batches }}$

NUCO350-30BI

NUCO350-37BI

NUCO350-29BI

LEU01-15I

LEU01-23I

LEU01-26I

LEU01-41I

TRISO-coated batches

LEU01-21T

LEU01-35T

LEU01-27T

LEU01-45T 


\section{Summary for Baseline Buffer Qualification Run - NUCO350-25B}

\begin{tabular}{|c|c|c|c|}
\hline Coating Run No. & & & NUCO350-25B \\
\hline Description: & \multicolumn{3}{|c|}{ Baseline processing conditions for Buffer layer } \\
\hline Procedure: & \multicolumn{3}{|c|}{ AGR-COAT-SOP-01, Rev. 1} \\
\hline Kernel Lot No. & \multicolumn{3}{|c|}{ NUCO350-25 } \\
\hline Operator: & \multicolumn{3}{|r|}{ R. A. Lowden } \\
\hline Date: & \multicolumn{3}{|c|}{$06 / 17 / 2005$} \\
\hline Data Location: & \multicolumn{3}{|c|}{ B002249, Coating Log, Volume 1, pp. $202-212$} \\
\hline Kernel Batch Wt. & \multicolumn{3}{|c|}{$62.11 \mathrm{~g}$} \\
\hline \multirow{2}{*}{ Coated Particle Batch Wt. } & \multicolumn{3}{|r|}{$81.33 \mathrm{~g}$} \\
\hline & AGR-1 Parameter & & As-Processed \\
\hline \multicolumn{4}{|l|}{ Buffer } \\
\hline Coating gases & \multicolumn{2}{|l|}{$\mathrm{C}_{2} \mathrm{H}_{2}+\mathrm{Ar}$} & $\mathrm{C}_{2} \mathrm{H}_{2}+\mathrm{Ar}$ \\
\hline TGF & \multirow{2}{*}{\multicolumn{2}{|c|}{$0.60 \pm 0.10$}} & $8530 \mathrm{sccm}$ \\
\hline CGF & & & 0.61 \\
\hline Temperature & \multicolumn{2}{|l|}{$1450 \pm 25^{\circ} \mathrm{C}$} & $1450^{\circ} \mathrm{C}$ \\
\hline Time & \multicolumn{2}{|l|}{$1450 \pm 25^{\circ} \mathrm{C}$} & $5 \mathrm{~min}$ \\
\hline \multicolumn{4}{|l|}{ IPyC } \\
\hline Coating gases & $\mathrm{Ar}+\mathrm{C}_{2} \mathrm{H}_{2}+\mathrm{C}_{3} \mathrm{H}_{6}$ & & \\
\hline TGF & & & \\
\hline CGF & $0.30 \pm 0.03$ & & \\
\hline CGR & $0.85 \pm 0.085$ & & \\
\hline Temperature & $1265 \pm 25^{\circ} \mathrm{C}$ & & \\
\hline Time & & & \\
\hline $\mathrm{SiC}$ & & & \\
\hline Coating gases & $\mathrm{H}_{2}+\mathrm{MTS}$ & & \\
\hline TGF & & & \\
\hline CGF & $0.015 \pm 0.005$ & & \\
\hline Temperature & $1500 \pm 25^{\circ} \mathrm{C}$ & & \\
\hline Time & & & \\
\hline OPyC & & & \\
\hline Coating gases & $\mathrm{Ar}+\mathrm{C}_{2} \mathrm{H}_{2}+\mathrm{C}_{3} \mathrm{H}_{6}$ & & \\
\hline TGF & & & \\
\hline CGF & $0.30 \pm 0.03$ & & \\
\hline CGR & $0.85 \pm 0.085$ & & \\
\hline Temperature & $1290 \pm 40^{\circ} \mathrm{C}$ & & \\
\hline Time & & & \\
\hline Comments/Notes: & & & \\
\hline Operator: & & Date: & 6 \\
\hline QAS: & & Date: & $3 / 30 / 06$ \\
\hline
\end{tabular}




\section{Summary for Baseline Buffer Qualification Run - NUCO350-36B}

\begin{tabular}{|c|c|c|c|}
\hline Coating Run No. & & & NUCO350-36B \\
\hline Description: & \multicolumn{3}{|c|}{ Baseline processing conditions for Buffer layer } \\
\hline Procedure: & \multicolumn{3}{|c|}{ AGR-COAT-SOP-01, Rev. 1} \\
\hline Kernel Lot No. & \multicolumn{3}{|c|}{ NUCO350-36 } \\
\hline Operator: & \multicolumn{3}{|c|}{ R. A. Lowden } \\
\hline Date: & \multicolumn{3}{|r|}{$06 / 17 / 2005$} \\
\hline Data Location: & \multicolumn{3}{|c|}{ B002249, Coating Log, Volume 1, pp. $214-223$} \\
\hline Kernel Batch Wt. & \multicolumn{3}{|c|}{$61.97 \mathrm{~g}$} \\
\hline \multirow[t]{2}{*}{ Coated Particle Batch Wt. } & \multicolumn{3}{|r|}{$81.50 \mathrm{~g}$} \\
\hline & AGR-1 Parameter & & As-Processed \\
\hline \multicolumn{4}{|l|}{ Buffer } \\
\hline Coating gases & \multicolumn{2}{|l|}{$\mathrm{C}_{2} \mathrm{H}_{2}+\mathrm{Ar}$} & $\mathrm{C}_{2} \mathrm{H}_{2}+\mathrm{Ar}$ \\
\hline TGF & \multirow{2}{*}{\multicolumn{2}{|c|}{$0.60 \pm 0.10$}} & $8530 \mathrm{sccm}$ \\
\hline CGF & & & 0.61 \\
\hline Temperature & \multicolumn{2}{|l|}{$1450 \pm 25^{\circ} \mathrm{C}$} & $1450^{\circ} \mathrm{C}$ \\
\hline Time & & $5 \mathrm{~min}$ \\
\hline \multicolumn{4}{|l|}{ IPyC } \\
\hline Coating gases & $\mathrm{Ar}+\mathrm{C}_{2} \mathrm{H}_{2}+\mathrm{C}_{3} \mathrm{H}_{6}$ & & \\
\hline TGF & & & \\
\hline CGF & $0.30 \pm 0.03$ & & \\
\hline CGR & $0.85 \pm 0.085$ & & \\
\hline Temperature & $1265 \pm 25^{\circ} \mathrm{C}$ & & \\
\hline Time & & & \\
\hline $\mathrm{SiC}$ & & & \\
\hline Coating gases & $\mathrm{H}_{2}+\mathrm{MTS}$ & & \\
\hline TGF & & & \\
\hline CGF & $0.015 \pm 0.005$ & & \\
\hline Temperature & $1500 \pm 25^{\circ} \mathrm{C}$ & & \\
\hline Time & & & \\
\hline OPyC & & & \\
\hline Coating gases & $\mathrm{Ar}+\mathrm{C}_{2} \mathrm{H}_{2}+\mathrm{C}_{3} \mathrm{H}_{6}$ & & \\
\hline TGF & & & \\
\hline CGF & $0.30 \pm 0.03$ & & \\
\hline CGR & $0.85 \pm 0.085$ & & \\
\hline Temperature & $1290 \pm 40^{\circ} \mathrm{C}$ & & \\
\hline Time & & & \\
\hline Comments/Notes: & & & \\
\hline Operator: & ـ & Date: & \\
\hline QAS: & & Date: & $3 / 30 / 06$ \\
\hline
\end{tabular}




\section{Summary for Baseline Buffer Qualification Run - NUCO350-54B}

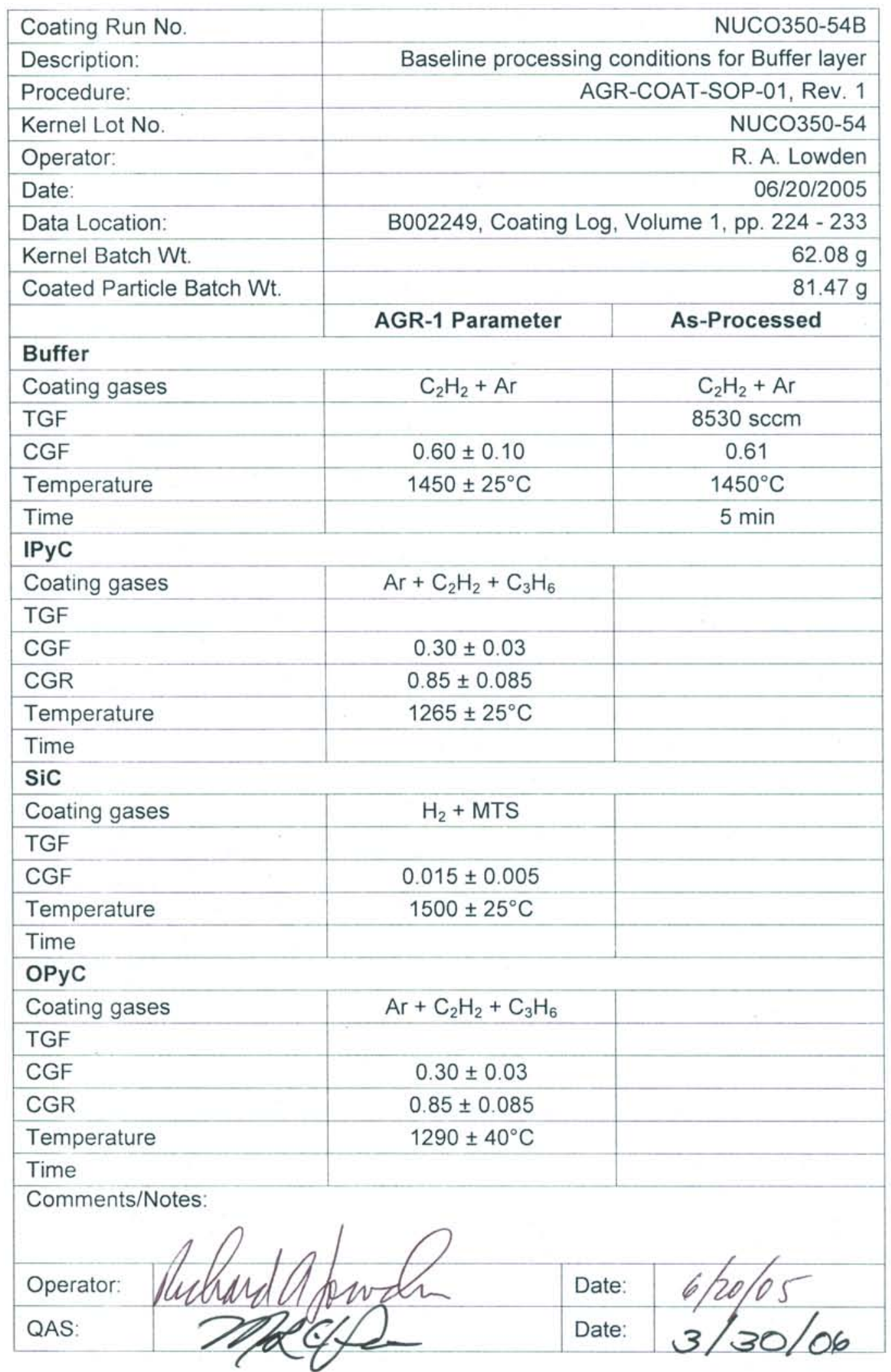




\section{Summary for Baseline Buffer Qualification Run - LEU01-16B}

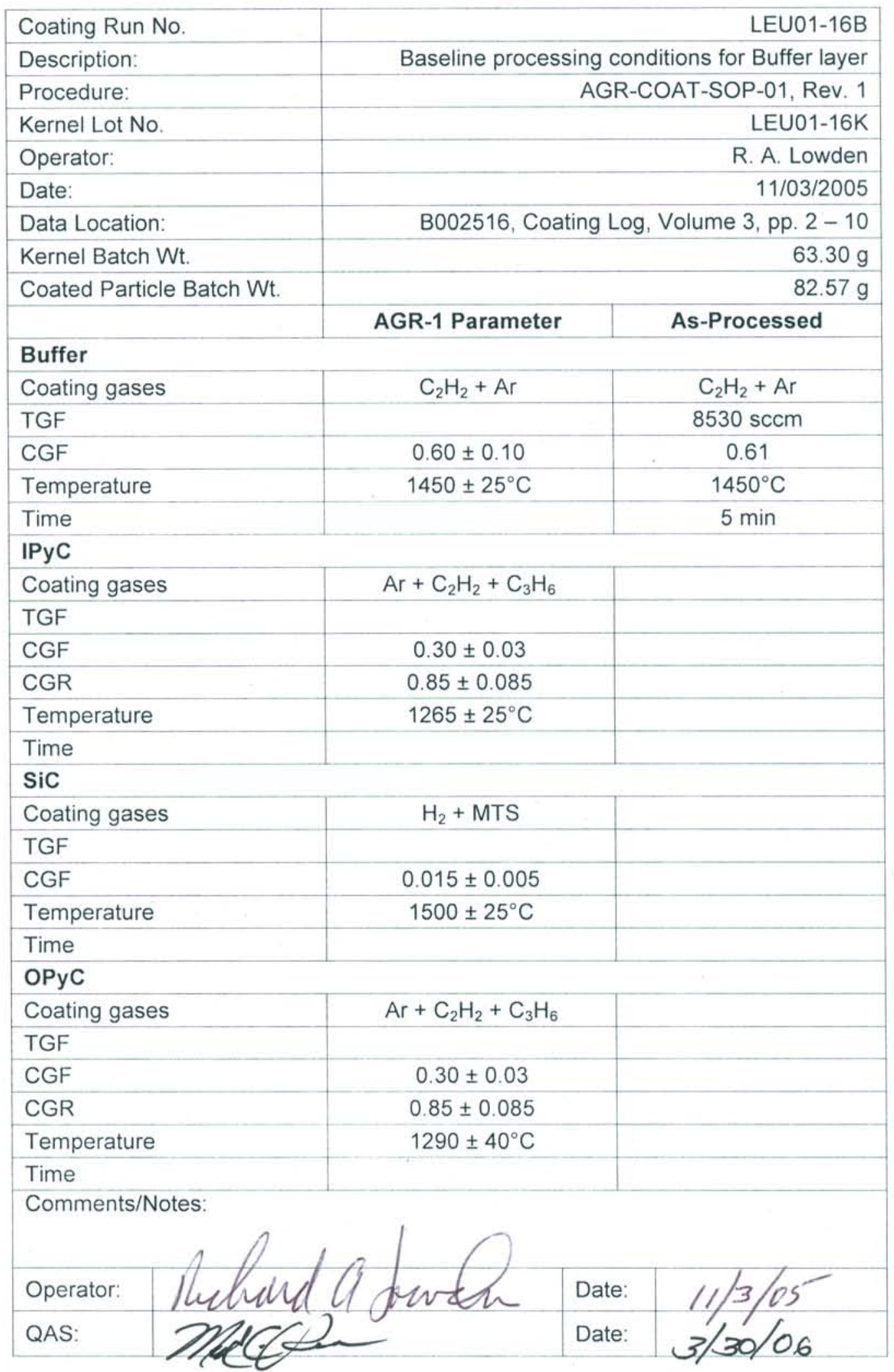


Summary for Baseline IPyC Qualification Run - NUCO350-30BI

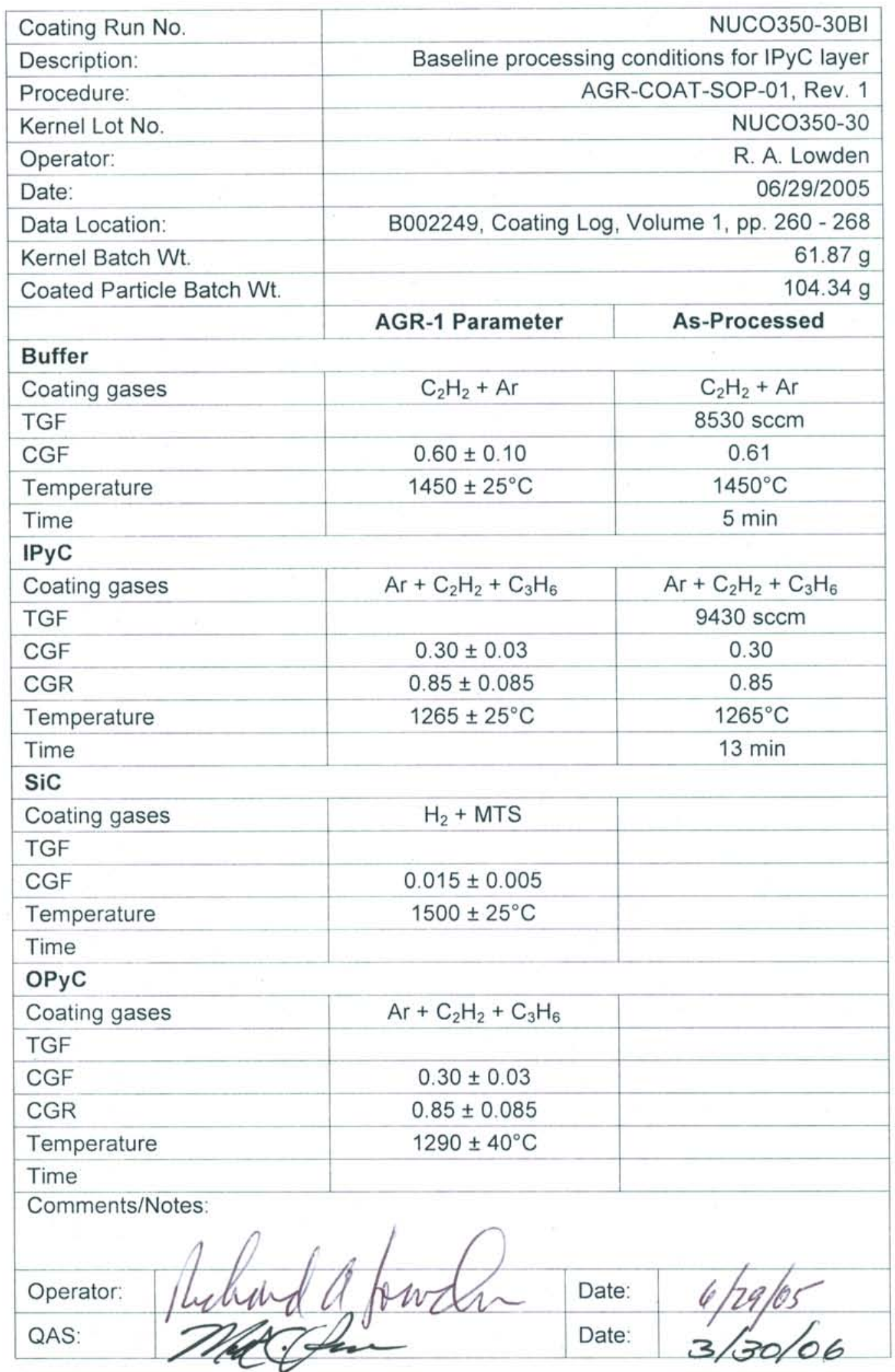




\section{Summary for Baseline IPyC Qualification Run - NUCO350-37BI}

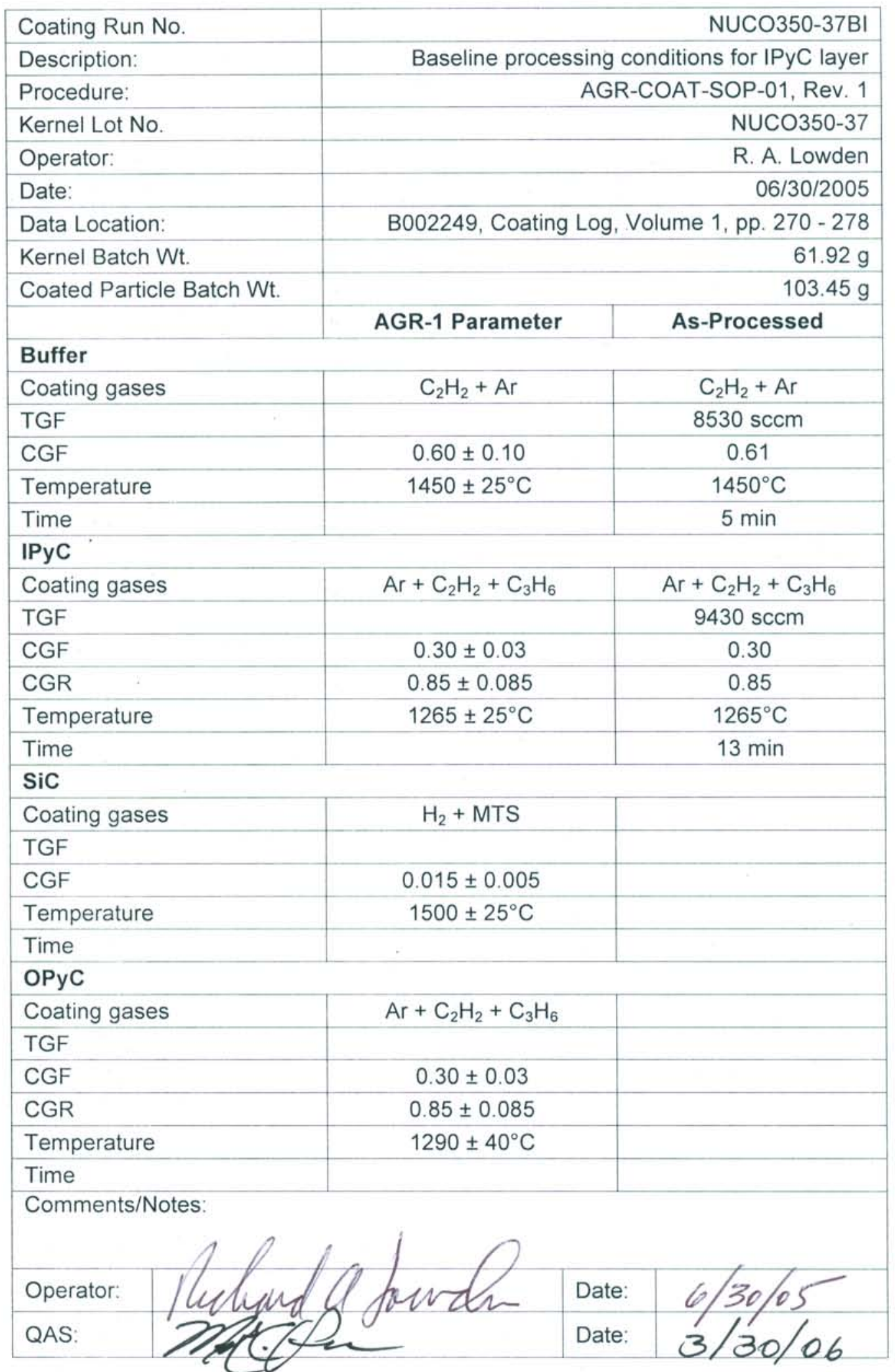




\section{Summary for Baseline IPyC Qualification Run - NUCO350-29BI}

\begin{tabular}{|c|c|c|c|}
\hline Coating Run No. & & & NUCO350-29BI \\
\hline Description: & \multicolumn{3}{|c|}{ Baseline processing conditions for IPyC layer } \\
\hline Procedure: & \multicolumn{3}{|c|}{ AGR-COAT-SOP-01, Rev. 1} \\
\hline Kernel Lot No. & \multicolumn{3}{|c|}{ NUCO350-29 } \\
\hline Operator: & \multicolumn{3}{|r|}{ R. A. Lowden } \\
\hline Date: & \multicolumn{3}{|r|}{$07 / 01 / 2005$} \\
\hline Data Location: & \multicolumn{3}{|c|}{ B002249, Coating Log, Volume 1, pp. $279-287$} \\
\hline \multirow{3}{*}{ Coated Particle Batch Wt. } & \multicolumn{3}{|c|}{$62.52 \mathrm{~g}$} \\
\hline & \multicolumn{3}{|r|}{$105.56 \mathrm{~g}$} \\
\hline & AGR-1 Parameter & & As-Processed \\
\hline \multicolumn{4}{|l|}{ Buffer } \\
\hline Coating gases & \multicolumn{2}{|l|}{$\mathrm{C}_{2} \mathrm{H}_{2}+\mathrm{Ar}$} & $\mathrm{C}_{2} \mathrm{H}_{2}+\mathrm{Ar}$ \\
\hline TGF & & & $8530 \mathrm{sccm}$ \\
\hline CGF & \multicolumn{2}{|l|}{$0.60 \pm 0.10$} & 0.61 \\
\hline Temperature & \multicolumn{2}{|l|}{$1450 \pm 25^{\circ} \mathrm{C}$} & $1450^{\circ} \mathrm{C}$ \\
\hline Time & & & $5 \mathrm{~min}$ \\
\hline \multicolumn{4}{|l|}{ IPyC } \\
\hline Coating gases & \multicolumn{2}{|l|}{$\mathrm{Ar}+\mathrm{C}_{2} \mathrm{H}_{2}+\mathrm{C}_{3} \mathrm{H}_{6}$} & $\mathrm{Ar}+\mathrm{C}_{2} \mathrm{H}_{2}+\mathrm{C}_{3} \mathrm{H}_{6}$ \\
\hline TGF & & & $9430 \mathrm{sccm}$ \\
\hline CGF & $0.30 \pm 0.03$ & & 0.30 \\
\hline CGR & $0.85 \pm 0.085$ & & 0.85 \\
\hline Temperature & $1265 \pm 25^{\circ} \mathrm{C}$ & & $1265^{\circ} \mathrm{C}$ \\
\hline Time & & & $13 \mathrm{~min}$ \\
\hline $\mathrm{SiC}$ & & & \\
\hline Coating gases & $\mathrm{H}_{2}+\mathrm{MTS}$ & & \\
\hline TGF & & & \\
\hline CGF & $0.015 \pm 0.005$ & & \\
\hline Temperature & $1500 \pm 25^{\circ} \mathrm{C}$ & & \\
\hline Time & & & \\
\hline OPyC & & & \\
\hline Coating gases & $\mathrm{Ar}+\mathrm{C}_{2} \mathrm{H}_{2}+\mathrm{C}_{3} \mathrm{H}_{6}$ & & \\
\hline TGF & & & \\
\hline CGF & $0.30 \pm 0.03$ & & \\
\hline CGR & $0.85 \pm 0.085$ & & \\
\hline Temperature & $1290 \pm 40^{\circ} \mathrm{C}$ & & \\
\hline Time & & & \\
\hline Comments/Notes: & & & \\
\hline Operator: & Un & Date: & $7 / 1 / 105$ \\
\hline QAS: & & Date: & $3 / 30 / 06$ \\
\hline
\end{tabular}




\section{Summary for Baseline IPyC Qualification Run - LEU01-15I}

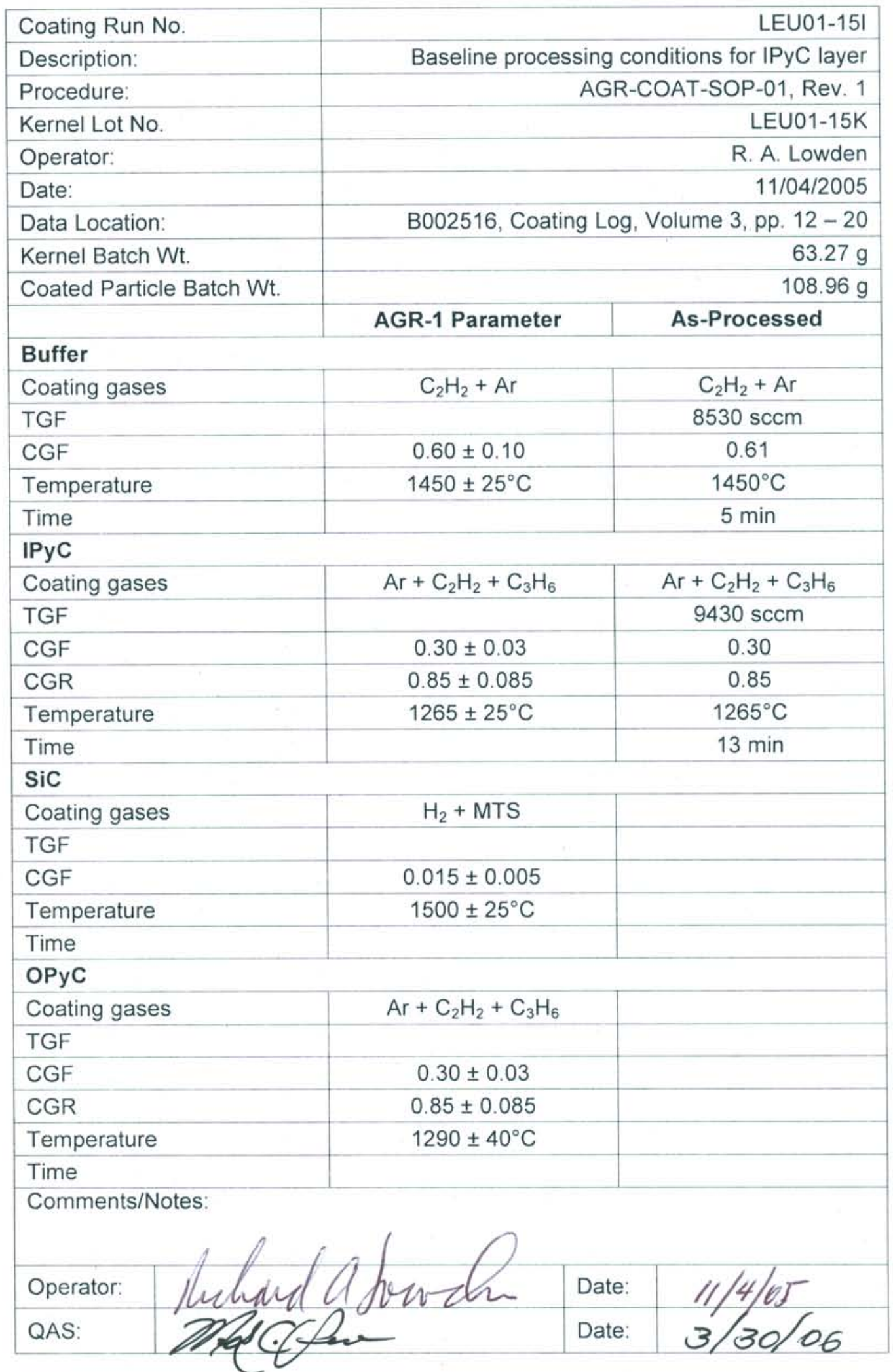




\section{Summary for Variant \#3b Coating Run - LEU01-23I}

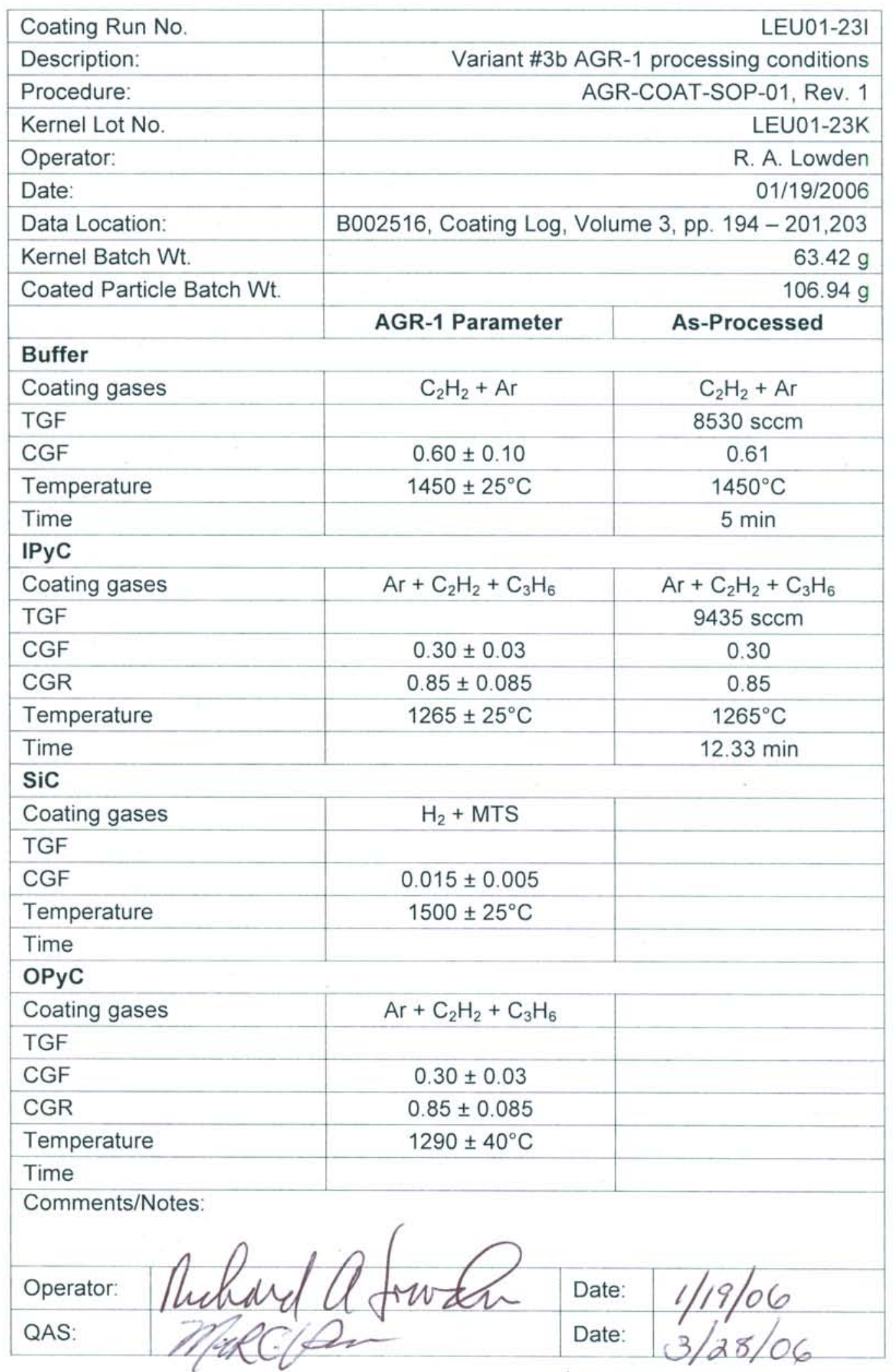




\section{Summary for Variant \#3b Coating Run - LEU01-26I}

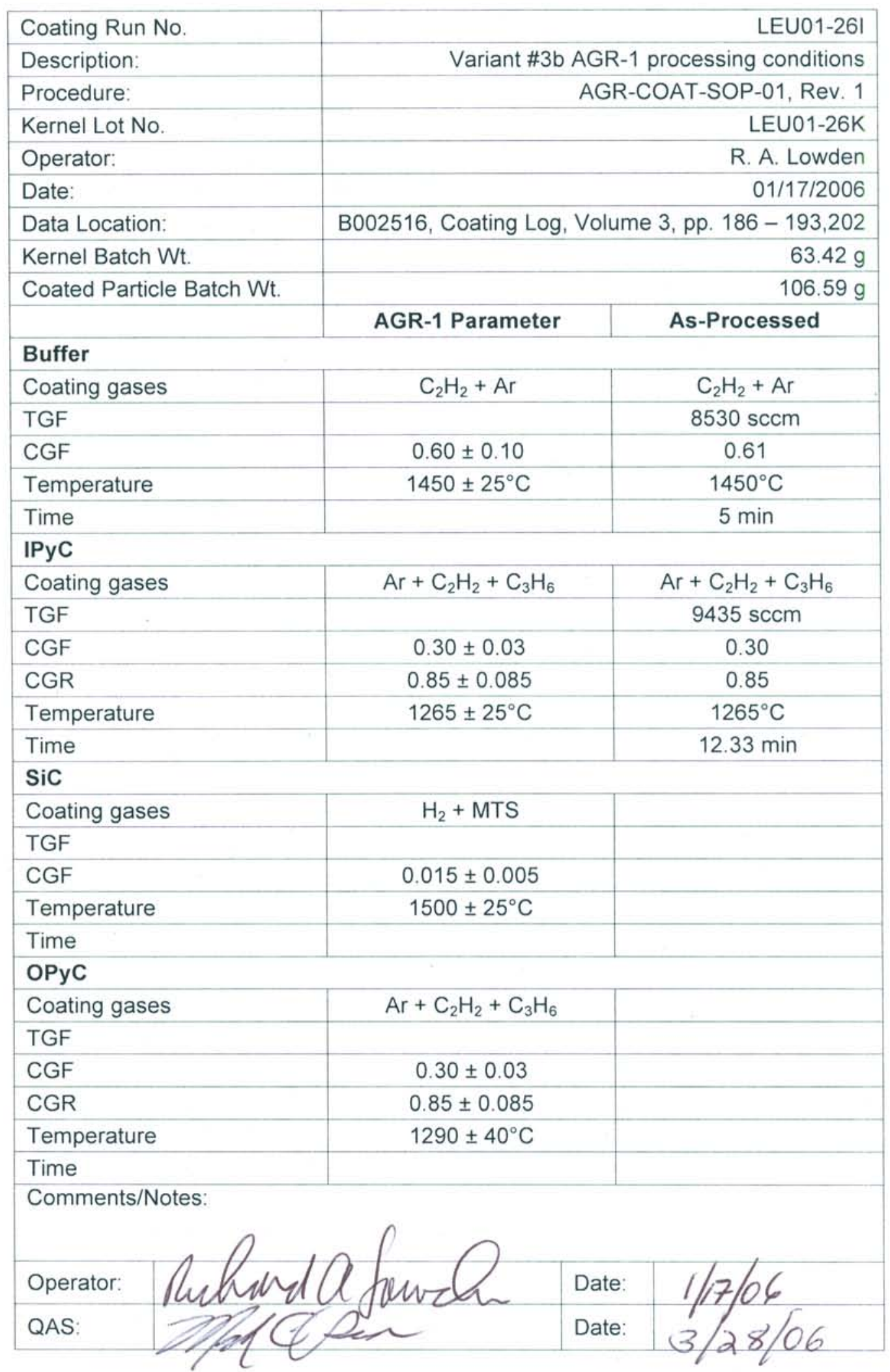




\section{Summary for Variant \#3b Coating Run - LEU01-41I}

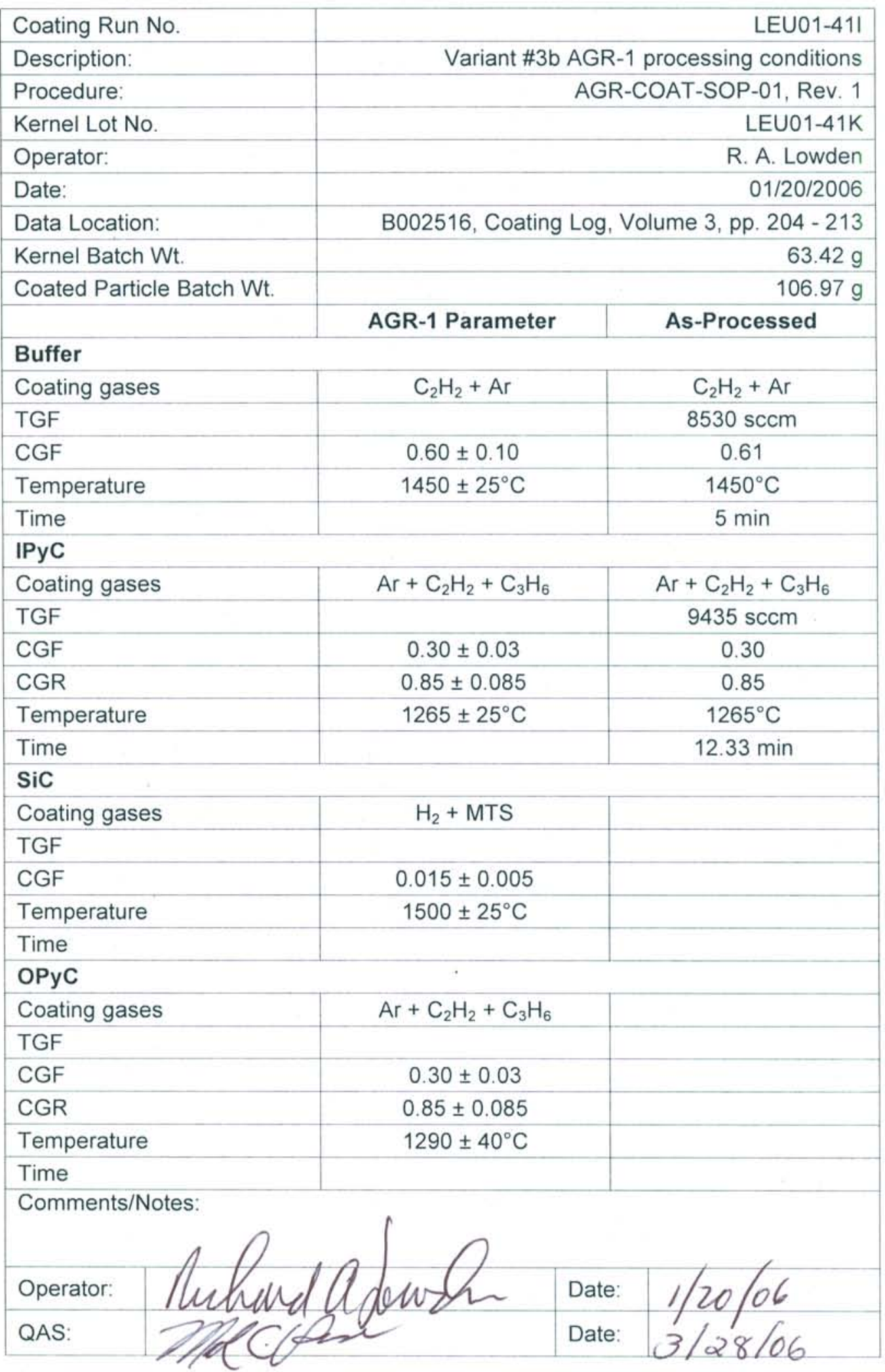




\section{Summary for Baseline Coating Run - LEU01-21T}

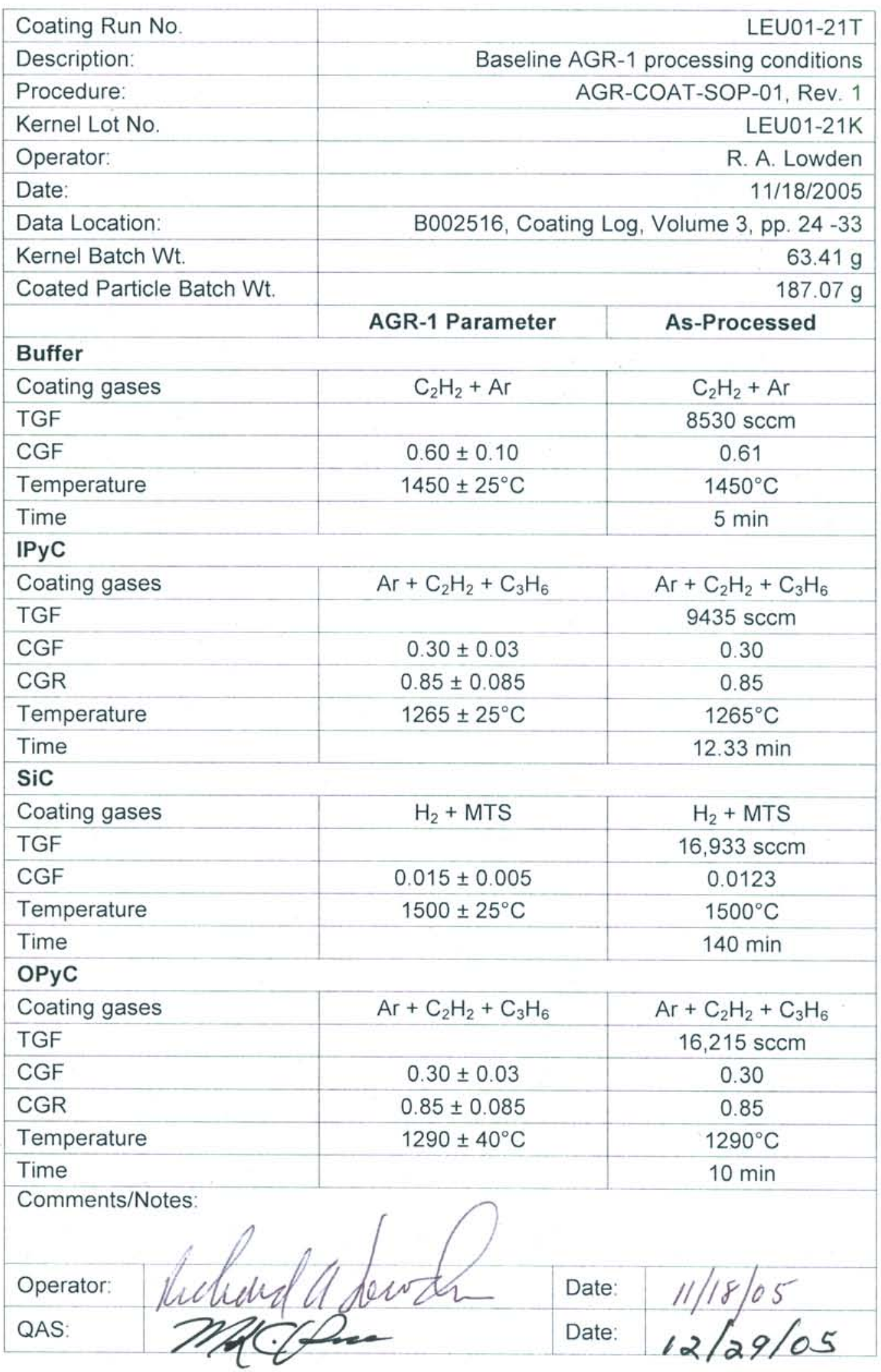




\section{Summary for Baseline Coating Run - LEU01-35T}

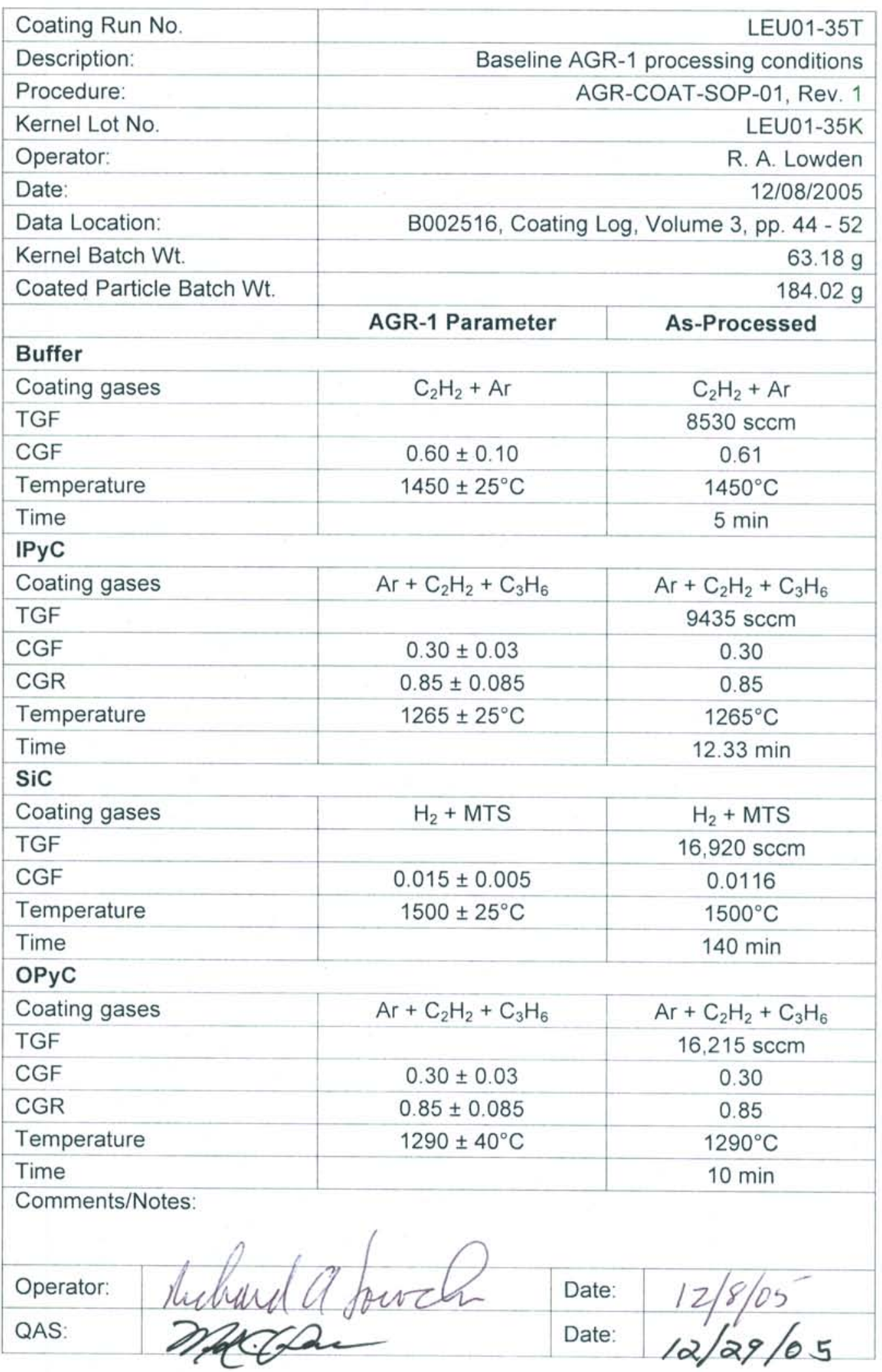




\section{Summary for Baseline Coating Run - LEU01-27T}

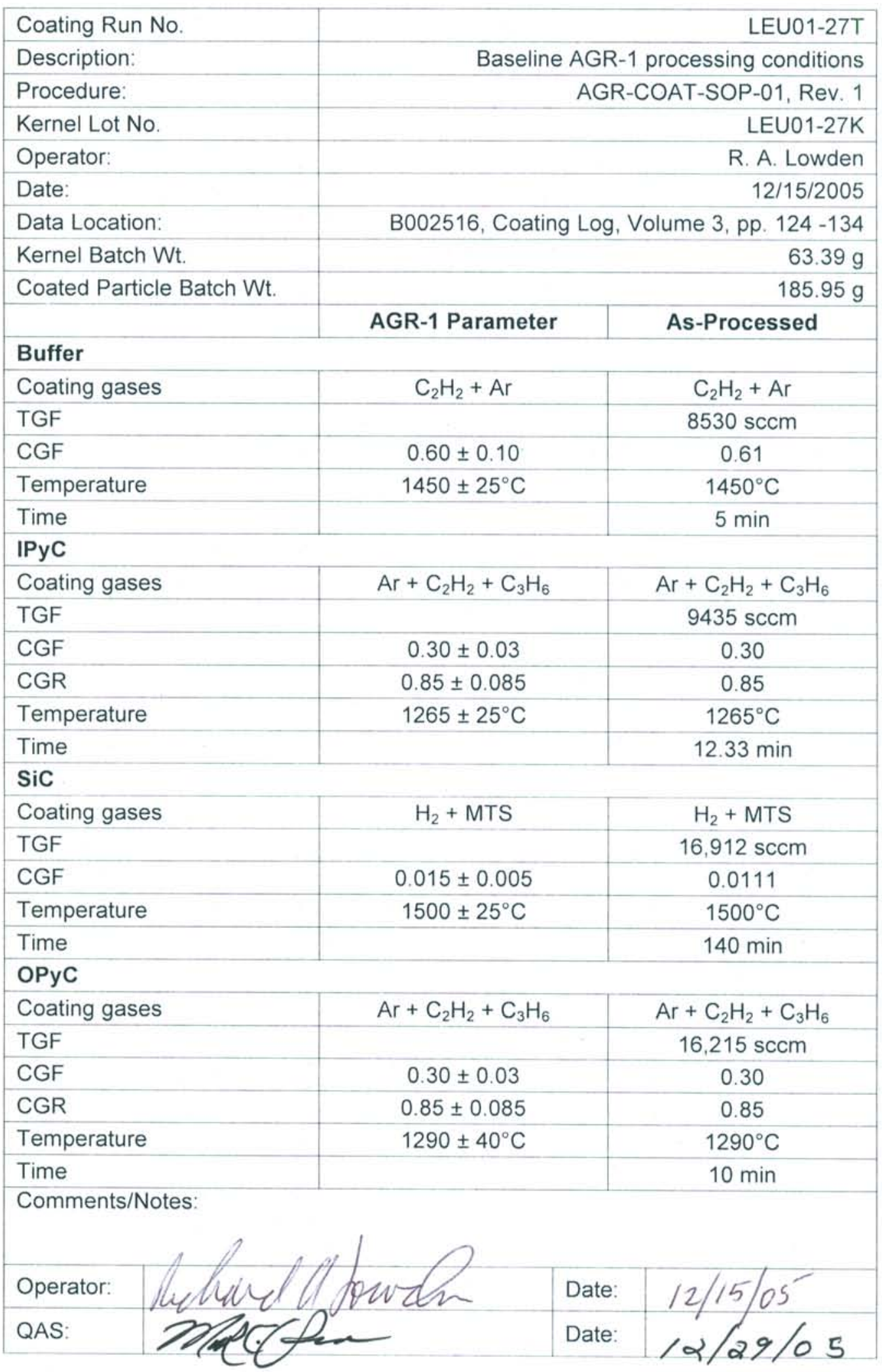




\section{Summary for Baseline Coating Run - LEU01-45T}

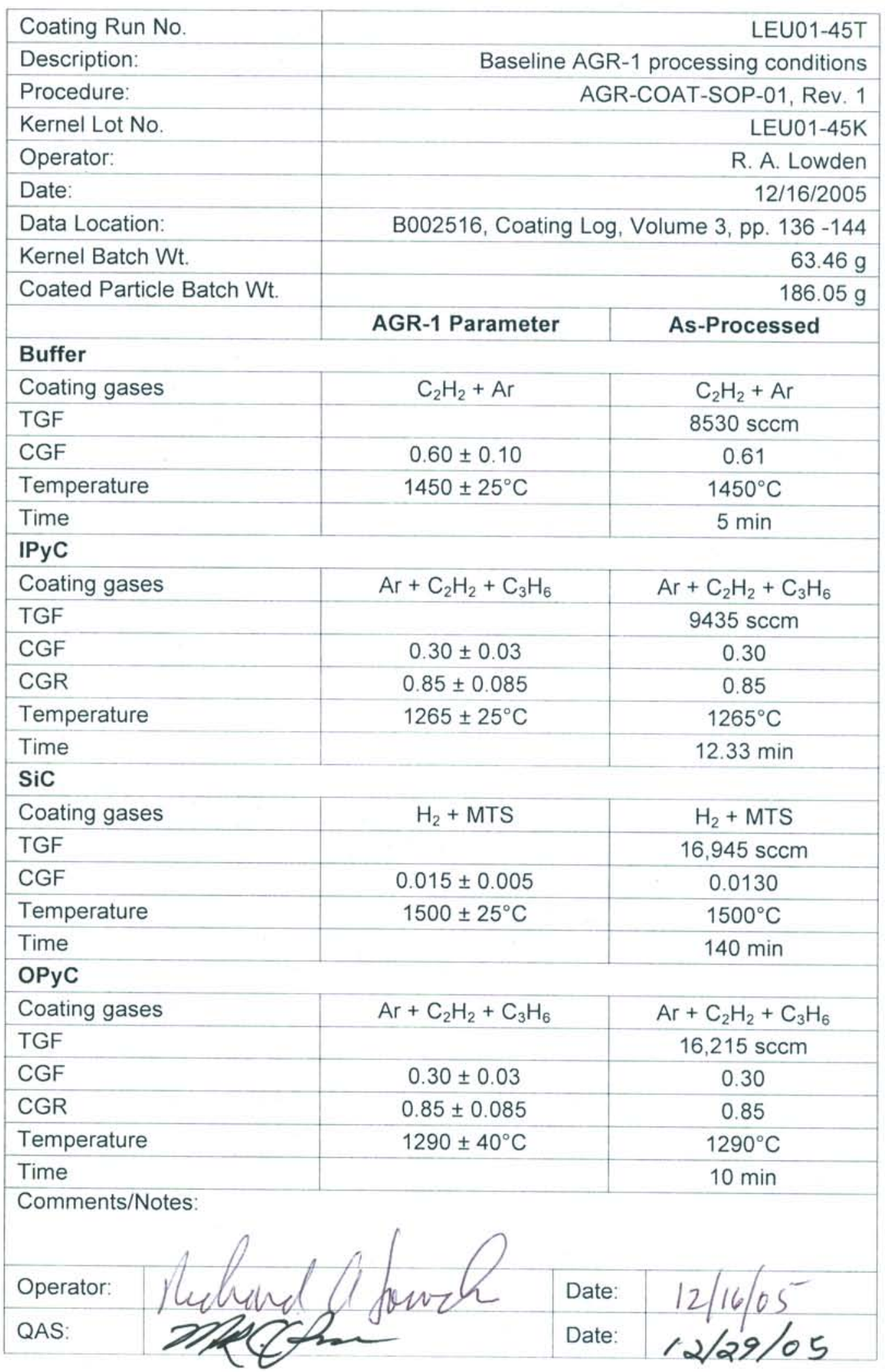




\section{Classification of coated particles}

Fully-coated batches of particles were sorted employing a sizing technique described in AGRROLLER-SOP-1, Rev. 0 and a tabling method described in AGR-TABLER-SOP-1, Rev. 1 as required in section 5.1 of the AGR-1 Fuel Product Specification and Characterization Guidance document INL EDF-4380, Rev. 6. The purpose of this classification is to remove aspherical particles and particles outside a specified diameter range. Details regarding the application of the procedures to the classification of coated particle batches can be found in the logbooks referenced on the summary sheets.

Particles can be sized using a set of sieves, with diameter ranges and limits determined by the selected mesh openings. As an alternative to sieving, the roller technique uses rotating sloped rollers with a diverging gap to size classify particles. Particles are fed onto the gap between the rollers. The rollers are tilted or angled downward away from the feed point to create an inclined track. The rollers rotate with an upward and outward motion. A particle travels down the gradually widening gap until it reaches a point equal to its diameter upon which it drops through the gap into a collection bin. The gap between the rollers is adjusted to separate different sizes of particles or to classify particles within a specific range of diameters. A schematic illustrating the relationship between roller gap and particle size is shown in Figure 4.1.

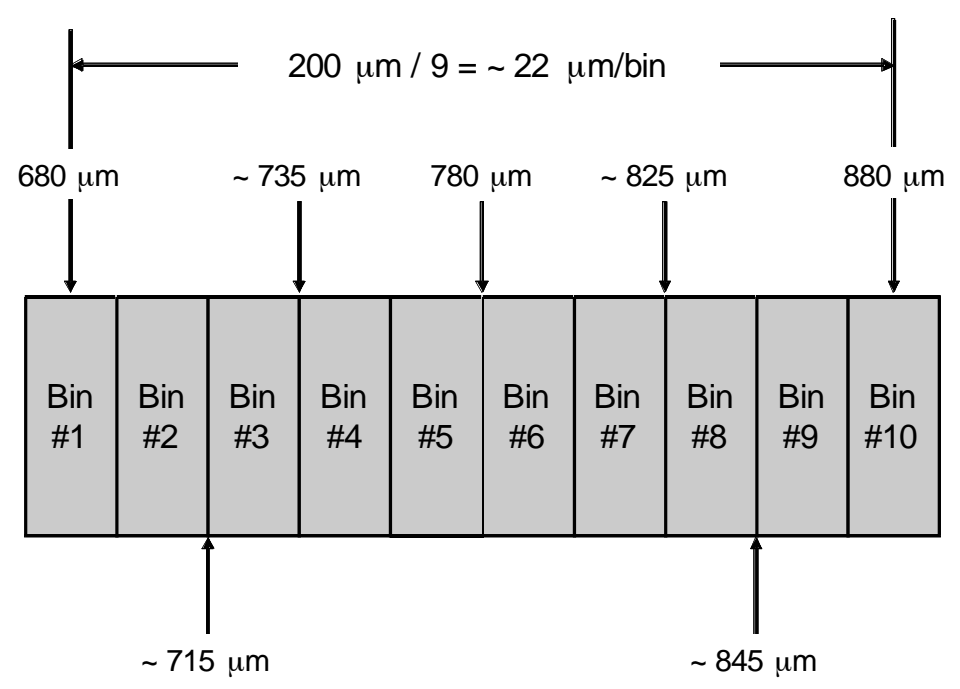

Figure 4.1. Schematic of the collection bins and roller spacing showing relationship between roller gap and particle size.

From EDF-4380, Rev 6, section 5.1.1, Sieving or Rolling to Remove Undersize and Oversize Particles, "Each batch of coated particles shall be double-sieved with electroformed sieves to remove all particles that pass through a $700 \mu \mathrm{m}$ sieve and all particles that do not pass through an $850 \mu \mathrm{m}$ sieve. . . . Alternately, the particles can be size-classified using a roller micrometer that achieves results equivalent to or better than sieving." To size separate the batches of coated 
particles, the gaps between the rollers above bins \#1 and \#10 were set at 680 and $880 \mu \mathrm{m}$, respectively, producing the size distribution shown in the figure. Only particles from Bins \#3 $\# 8$, with a particle size range of 715 to $845 \mu \mathrm{m}$, were retained with the majority of the particles being from Bins \#4 through \#7 with a nominal particle size range 735 to $825 \mu \mathrm{m}$.

The following pages contain records of the classification performed on the TRISO-coated particle batches associated with the LEU01-46T coated particle composite.

TRISO batches

LEU01-21T

LEU01-35T

LEU01-27T

LEU01-45T 


\section{Sizing \& Removal of Aspherical Particles for LEU01-21T}

Summary for Sizing of Kernels or Coated Particles

\begin{tabular}{|c|c|c|c|c|}
\hline \multicolumn{2}{|l|}{ Procedure: } & & \multicolumn{2}{|c|}{ AGR-ROLLER-SOP-01, Rev. 0} \\
\hline \multicolumn{2}{|l|}{ Operator: } & & & R. A. Lowden \\
\hline \multicolumn{2}{|c|}{ Kernel/Coated Particle ID: } & & & LEU01-21T \\
\hline \multicolumn{2}{|c|}{ Kernel/Coated Particle Description: } & \multicolumn{3}{|c|}{ Baseline, full TRISO on $350 \mu \mathrm{m}$ LEUCO } \\
\hline \multicolumn{2}{|l|}{ Data Location: } & \multicolumn{3}{|c|}{ B002163, Sizing \& Tabling, p. 31} \\
\hline Date & $\begin{array}{c}\text { Batch Weight } \\
\text { (g) }\end{array}$ & $\begin{array}{l}\text { Sized Batch } \\
\text { Weight } \\
\text { (g) }\end{array}$ & $\begin{array}{l}\text { Scrap } \\
\text { (g) }\end{array}$ & $\begin{array}{l}\text { Loss } \\
\text { (g) }\end{array}$ \\
\hline $11 / 22 / 2005$ & 187.07 & 178.13 & 8.90 & $(0.04)$ \\
\hline \multicolumn{5}{|l|}{ Comments: } \\
\hline Operator: & & & Date: & $1 / 22 / 05$ \\
\hline QAS: & & & Date: & \\
\hline
\end{tabular}

\section{Summary for Tabling of Kernels or Coated Particles}

\begin{tabular}{|c|c|c|c|c|}
\hline \multicolumn{2}{|l|}{ Procedure: } & & \multicolumn{2}{|c|}{ AGR-TABLE-SOP-01, Rev. 1} \\
\hline \multicolumn{2}{|l|}{ Operator: } & & \multicolumn{2}{|r|}{ J. H. Miller } \\
\hline \multicolumn{2}{|c|}{ Kernel/Coated Particle ID: } & & \multicolumn{2}{|r|}{ LEU01-21T } \\
\hline \multicolumn{2}{|c|}{ Kernel/Coated Particle Description: } & \multicolumn{3}{|c|}{ Baseline, full TRISO on $350 \mu \mathrm{m}$ LEUCO } \\
\hline \multicolumn{2}{|l|}{ Data Location: } & \multicolumn{3}{|c|}{ B002163, Sizing \& Tabling, p. 32} \\
\hline Date & $\begin{array}{l}\text { Batch Weight } \\
\text { (g) }\end{array}$ & $\begin{array}{l}\text { Tabled Batch } \\
\text { Weight } \\
\text { (g) }\end{array}$ & $\begin{array}{l}\text { Scrap } \\
\text { (g) }\end{array}$ & $\begin{array}{l}\text { Loss } \\
\text { (g) }\end{array}$ \\
\hline $11 / 23 / 2005$ & 178.13 & 177.03 & 1.10 & 0.00 \\
\hline \multicolumn{5}{|l|}{ Comments: } \\
\hline Operator: & & & Date: & $11-23-05$ \\
\hline QAS: & & & Date: & \\
\hline
\end{tabular}




\section{Sizing \& Removal of Aspherical Particles for LEU01-35T}

Summary for Sizing of Kernels or Coated Particles

\begin{tabular}{|c|c|c|c|c|}
\hline \multicolumn{2}{|l|}{ Procedure: } & & \multicolumn{2}{|c|}{ AGR-ROLLER-SOP-01, Rev. 0} \\
\hline \multicolumn{2}{|l|}{ Operator: } & & & J. H. Miller \\
\hline \multicolumn{2}{|c|}{ Kernel/Coated Particle ID: } & & & LEU01-35T \\
\hline \multicolumn{2}{|c|}{ Kernel/Coated Particle Description: } & \multicolumn{3}{|c|}{ Baseline, full TRISO on $350 \mu \mathrm{m}$ LEUCO } \\
\hline \multicolumn{2}{|l|}{ Data Location: } & \multicolumn{3}{|c|}{ B002163, Sizing \& Tabling, p. 38} \\
\hline Date & $\begin{array}{c}\text { Batch Weight } \\
\text { (g) }\end{array}$ & $\begin{array}{l}\text { Sized Batch } \\
\text { Weight } \\
(\mathrm{g})\end{array}$ & $\begin{array}{l}\text { Scrap } \\
\text { (g) }\end{array}$ & $\begin{array}{l}\text { Loss } \\
\text { (g) }\end{array}$ \\
\hline $12 / 09 / 2005$ & 184.02 & 172.49 & 11.52 & $(0.01)$ \\
\hline \multicolumn{5}{|l|}{ Comments: } \\
\hline Operator: & Hanest. I & Or & Date: & $12-9-05$ \\
\hline QAS: & & & Date: & $3 / 30 / 06$ \\
\hline
\end{tabular}

\section{Summary for Tabling of Kernels or Coated Particles}

\begin{tabular}{|c|c|c|c|c|}
\hline \multicolumn{2}{|l|}{ Procedure: } & & \multicolumn{2}{|c|}{ AGR-TABLE-SOP-01, Rev. 1} \\
\hline \multicolumn{2}{|l|}{ Operator: } & & & J. H. Miller \\
\hline \multicolumn{2}{|c|}{ Kernel/Coated Particle ID: } & & & LEU01-35T \\
\hline \multicolumn{2}{|c|}{ Kernel/Coated Particle Description: } & \multicolumn{3}{|c|}{ Baseline, full TRISO on $350 \mu \mathrm{m}$ LEUCO } \\
\hline \multicolumn{2}{|l|}{ Data Location: } & \multicolumn{3}{|c|}{ B002163, Sizing \& Tabling, p. 39} \\
\hline Date & $\begin{array}{l}\text { Batch Weight } \\
\quad(\mathrm{g})\end{array}$ & $\begin{array}{l}\text { Tabled Batch } \\
\text { Weight } \\
\text { (g) }\end{array}$ & $\begin{array}{l}\text { Scrap } \\
\text { (g) }\end{array}$ & $\begin{array}{l}\text { Loss } \\
\text { (g) }\end{array}$ \\
\hline $12 / 09 / 2005$ & 172.49 & 165.06 & 7.43 & 0.00 \\
\hline \multicolumn{5}{|l|}{ Comments: } \\
\hline Operator: & e) 14 & las & Date: & $12-9.05$ \\
\hline QAS: & & & Date: & \\
\hline
\end{tabular}




\section{Sizing \& Removal of Aspherical Particles for LEU01-27T}

\section{Summary for Sizing of Kernels or Coated Particles}

\begin{tabular}{|c|c|c|c|c|}
\hline \multicolumn{2}{|l|}{ Procedure: } & & \multicolumn{2}{|c|}{ AGR-ROLLER-SOP-01, Rev. 0} \\
\hline \multicolumn{2}{|l|}{ Operator: } & & & R. A. Lowden \\
\hline \multicolumn{2}{|c|}{ Kernel/Coated Particle ID: } & & & LEU01-27T \\
\hline \multicolumn{2}{|c|}{ Kernel/Coated Particle Description: } & \multicolumn{3}{|c|}{ Baseline, full TRISO on $350 \mu \mathrm{m}$ LEUCO } \\
\hline \multicolumn{2}{|l|}{ Data Location: } & \multicolumn{3}{|c|}{ B002163, Sizing \& Tabling, p. 40} \\
\hline Date & $\begin{array}{c}\text { Batch Weight } \\
\text { (g) }\end{array}$ & $\begin{array}{l}\text { Sized Batch } \\
\text { Weight } \\
\text { (g) }\end{array}$ & $\begin{array}{l}\text { Scrap } \\
\text { (g) }\end{array}$ & $\begin{array}{l}\text { Loss } \\
\text { (g) }\end{array}$ \\
\hline $12 / 15 / 2005$ & 185.95 & 176.95 & 8.96 & $(0.04)$ \\
\hline \multicolumn{5}{|l|}{ Comments: } \\
\hline Operator: & & & Date: & $12 / 15 / 05$ \\
\hline QAS: & & & Date: & \\
\hline
\end{tabular}

\section{Summary for Tabling of Kernels or Coated Particles}

\begin{tabular}{|c|c|c|c|c|}
\hline \multicolumn{2}{|l|}{ Procedure: } & & \multicolumn{2}{|c|}{ AGR-TABLE-SOP-01, Rev. 1} \\
\hline \multicolumn{2}{|l|}{ Operator: } & & & R. A. Lowden \\
\hline \multicolumn{2}{|c|}{ Kernel/Coated Particle ID: } & & & LEU01-27T \\
\hline \multicolumn{2}{|c|}{ Kernel/Coated Particle Description: } & \multicolumn{3}{|c|}{ Baseline, full TRISO on $350 \mu \mathrm{m}$ LEUCO } \\
\hline \multicolumn{2}{|l|}{ Data Location: } & \multicolumn{3}{|c|}{ B002163, Sizing \& Tabling, p. 41} \\
\hline Date & $\begin{array}{c}\text { Batch Weight } \\
\text { (g) }\end{array}$ & $\begin{array}{l}\text { Tabled Batch } \\
\text { Weight } \\
\text { (g) }\end{array}$ & $\begin{array}{l}\text { Scrap } \\
\text { (g) }\end{array}$ & $\begin{array}{l}\text { Loss } \\
\text { (g) }\end{array}$ \\
\hline $12 / 16 / 2005$ & 176.98 & 170.19 & 6.77 & $(0.02)$ \\
\hline \multicolumn{5}{|l|}{ Comments: } \\
\hline Operator: & & & Date: & \\
\hline QAS: & & & Date: & \\
\hline
\end{tabular}




\section{Sizing \& Removal of Aspherical Particles for LEU01-45T}

Summary for Sizing of Kernels or Coated Particles

\begin{tabular}{|c|c|c|c|c|}
\hline \multicolumn{2}{|l|}{ Procedure: } & & \multicolumn{2}{|c|}{ AGR-ROLLER-SOP-01, Rev. 0} \\
\hline \multicolumn{2}{|l|}{ Operator: } & & & R. A. Lowden \\
\hline \multicolumn{2}{|c|}{ Kernel/Coated Particle ID: } & & & LEU01-45T \\
\hline \multicolumn{2}{|c|}{ Kernel/Coated Particle Description: } & \multicolumn{3}{|c|}{ Baseline, full TRISO on $350 \mu \mathrm{m}$ LEUCO } \\
\hline \multicolumn{2}{|l|}{ Data Location: } & \multicolumn{3}{|c|}{ B002163, Sizing \& Tabling, p. 42} \\
\hline Date & $\begin{array}{l}\text { Batch Weight } \\
\text { (g) }\end{array}$ & $\begin{array}{l}\text { Sized Batch } \\
\text { Weight } \\
\text { (g) }\end{array}$ & $\begin{array}{l}\text { Scrap } \\
\text { (g) }\end{array}$ & $\begin{array}{l}\text { Loss } \\
\text { (g) }\end{array}$ \\
\hline $12 / 20 / 2005$ & 186.05 & 177.60 & 8.49 & +0.04 \\
\hline \multicolumn{5}{|l|}{ Comments: } \\
\hline Operator: & & & Date: & 20105 \\
\hline QAS: & & & Date: & \\
\hline
\end{tabular}

Summary for Tabling of Kernels or Coated Particles

\begin{tabular}{|c|c|c|c|c|}
\hline \multicolumn{2}{|l|}{ Procedure: } & & \multicolumn{2}{|c|}{ AGR-TABLE-SOP-01, Rev. 1} \\
\hline \multicolumn{2}{|l|}{ Operator: } & & & R. A. Lowden \\
\hline \multicolumn{2}{|c|}{ Kernel/Coated Particle ID: } & & & LEU01-45T \\
\hline \multicolumn{2}{|c|}{ Kernel/Coated Particle Description: } & \multicolumn{3}{|c|}{ Baseline, full TRISO on $350 \mu \mathrm{m}$ LEUCO } \\
\hline \multicolumn{2}{|l|}{ Data Location: } & \multicolumn{3}{|c|}{ B002163, Sizing \& Tabling, p. 43} \\
\hline Date & $\begin{array}{l}\text { Batch Weight } \\
\text { (g) }\end{array}$ & $\begin{array}{l}\text { Tabled Batch } \\
\text { Weight } \\
\text { (g) }\end{array}$ & $\begin{array}{l}\text { Scrap } \\
\text { (g) }\end{array}$ & $\begin{array}{l}\text { Loss } \\
\text { (g). }\end{array}$ \\
\hline $12 / 21 / 2005$ & 177.60 & 170.51 & 7.07 & $(0.02)$ \\
\hline \multicolumn{5}{|l|}{ Comments: } \\
\hline Operator: & 111 & waten & Date: & $21 / 05$ \\
\hline QAS: & & & Date: & \\
\hline
\end{tabular}




\section{Blend of coated particle composites}

Four batches of TRISO-coated particles were blended into composite LEU01-46T. The mass of each batch added to the composite is shown in the following table.

\begin{tabular}{|c|c|}
\hline Batch ID & Amount added to LEU01-46T composite (g) \\
\hline LEU01-21T & 69.2704 \\
\hline LEU01-35T & 146.2983 \\
\hline LEU01-27T & 151.5947 \\
\hline LEU01-45T & 151.9880 \\
\hline \hline Total & 519.1514 \\
\hline
\end{tabular}

Three batches of buffer-coated particles were blended into composite NUCO350-58B. The mass of each batch added to the composite is shown in the following table.

\begin{tabular}{|c|c|}
\hline Batch ID & Amount added to NUCO350-58B composite (g) \\
\hline NUCO350-25B & 54.4356 \\
\hline NUCO350-36B & 55.8622 \\
\hline NUCO350-54B & 56.0934 \\
\hline \hline Total & 166.3912 \\
\hline
\end{tabular}

Three batches of buffer/IPyC-coated particles were blended into composite NUCO350-66BI. The mass of each batch added to the composite is shown in the following table.

\begin{tabular}{|c|c|}
\hline Batch ID & Amount added to NUCO350-66BI composite (g) \\
\hline NUCO350-30BI & 96.9421 \\
\hline NUCO350-37BI & 96.1568 \\
\hline NUCO350-29BI & 98.2502 \\
\hline \hline Total & 291.3491 \\
\hline
\end{tabular}




\section{Characterization of LEUCO kernel composite}

This section contains data on the kernel composite used for LEU01-46T. The data was obtained according to product inspection plan AGR-CHAR-PIP-01R1. Some of the kernel data in this section was used as input for subsequent measurements of coating properties (e.g., buffer density and burn-leach defects). This is only a partial analysis of the kernel composite and was not used for product acceptance. Characterization of the kernel composite for acceptance according to the specific requirements listed in section 4 of INL EDF-4380 is documented in the BWXT data package for G73D-20-69302. The BWXT kernel lot G73D-20-69302 was riffled into sublots for characterization and coating by ORNL and identified as LEU01-\#\# (where \#\# is a series of integers beginning with 01).

Note that the average kernel weights were remeasured using a revised procedure which improved the accuracy of the reported value by improving the randomness of the measured samples. The measured value of the LEUCO average kernel weight changed from $(2.47 \pm 0.01) \cdot 10^{-4}$ grams per kernel to $(2.418 \pm 0.006) \cdot 10^{-4}$ grams per kernel. On data report form DRF-15 for the measurement of average kernel envelope density, the average kernel weight is used to approximate the number of kernels in a sample from the weight of the sample. The approximate number of kernels in a sample is divided into the sample envelope volume to determine the average kernel envelope volume. The change in average kernel weight resulted in a significant change in the calculated average kernel envelope volume. The data in this section shows the new average kernel weight and average kernel envelope volume. Records showing the original values are included in section 16.

The following pages shows the inspection report form (IRF-01). Following IRF-01 are the individual data report forms for the measurements that were performed. 
ORNL/TM-2006/019, Rev. 1

Inspection Report Form IRF-01: BWXT LEUCO Kernel Composite 69302

Procedure: AGR-CHAR-PIP-01 Rev. 1

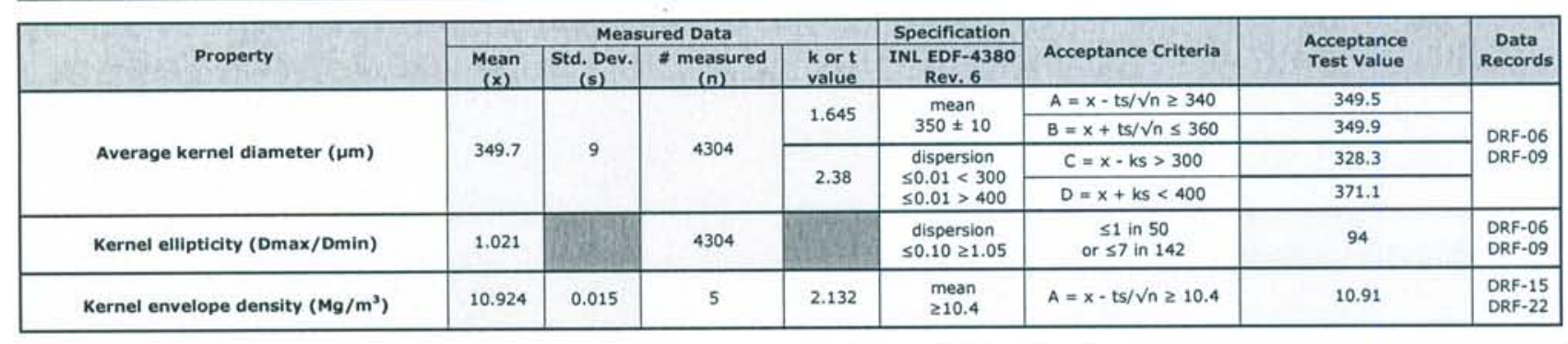

94 kernels with ellipticity $\geq 1.05$ out of 4304 kernels measured passes the dispersion specification acceptance criteria of $\leq 397$ in 4304 .

This composite would pass a control limit of $\geq 1.035$ at $10 \%$ tolerance limit with $95 \%$ confidence level.
This composite would pass a control limit of $\geq 1.05$ at $2.6 \%$ tolerance limit with $95 \%$ confidence level.

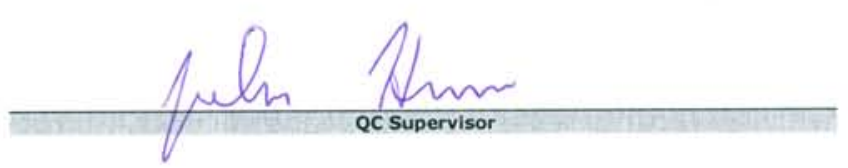

$$
\frac{2-16-06}{\text { Date }}
$$

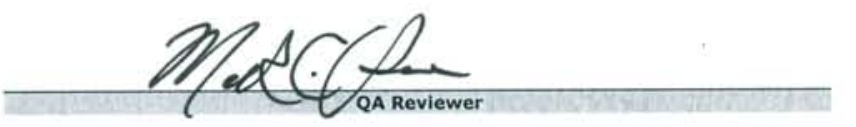

$3 / 24 / 06$

40 
Data Report Form DRF-06: Imaging of Kernel Diameter and Ellipticity Using an Optical Microscope System

\begin{tabular}{|c|c|}
\hline Procedure: & AGR-CHAR-DAM-06 Rev. 0 \\
\hline Operator: & Andrew Kercher \\
\hline Sample ID: & EEUEO350-01B renamed LEUOI-OIK-B \\
\hline Sample Description: & LEUCO kernel composite 69302 \\
\hline Folder name containing images: & 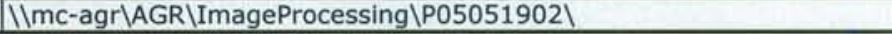 \\
\hline
\end{tabular}

\begin{tabular}{|r|l|}
\hline DMR Calibration Expiration Date: & $3 / 24 / 06$ \\
\hline Stage Micrometer Calibration Expiration Date: & $2 / 17 / 07$ \\
\hline Measured Value for $\mathbf{7 6 0} \mu \mathrm{m}$ in Stage Micrometer Image: & $760 . \mu \mathrm{m}$ \\
\hline
\end{tabular}

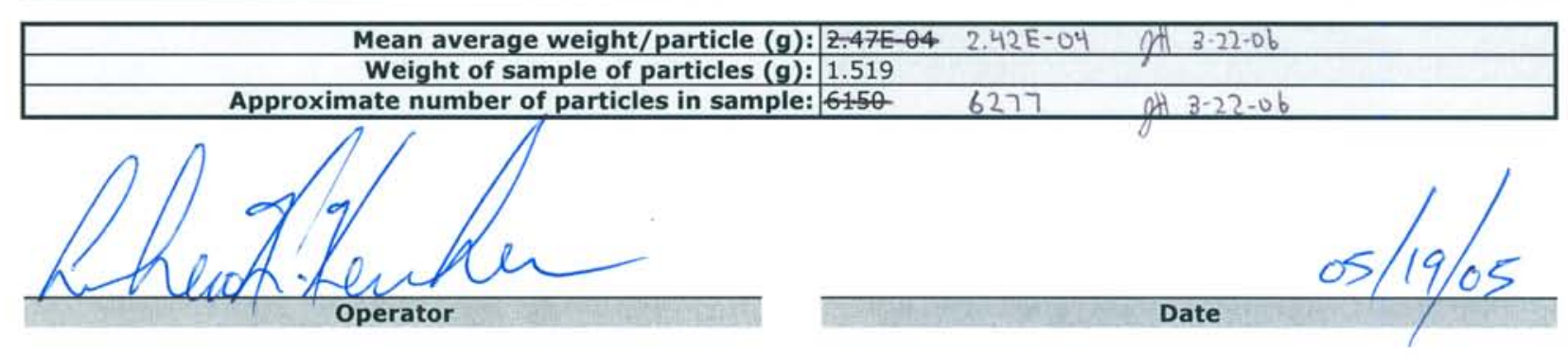


Data Report Form DRF-09A: Measurement of Kernel Diameter

Procedure: AGR-CHAR-DAM-09 Rev. 0

Operator: Andrew K. Kercher

Folder name containing images: (IImc-agr|AGR\ImageProcessing\P05051902

Sample ID: LEUEOJ50-O1B renamed LEVOI-OIK-B NA 4-17-O6

Sample Description: BWXT LEUCO kernel composite 69302

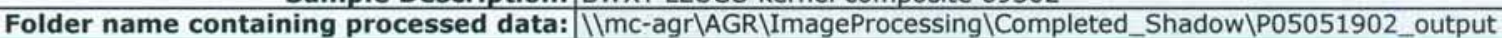

Number of kernels analyzed: 4304

Mean of the average diameter of each kernel $(\mu \mathrm{m}): 349.7$

Standard deviation in the average diameter of each kernel $(\mu \mathrm{m}): 9$

Distribution of the average kernel diameter (top binned)

\begin{tabular}{|rr|}
\hline Mean Diameter & Frequency \\
\hline$<300$ & 9 \\
305 & 2 \\
310 & 2 \\
315 & 1 \\
320 & 2 \\
325 & 2 \\
330 & 18 \\
335 & 143 \\
340 & 366 \\
345 & 666 \\
350 & 1060 \\
355 & 946 \\
360 & 572 \\
365 & 317 \\
370 & 128 \\
375 & 45 \\
380 & 18 \\
385 & 4 \\
390 & 3 \\
395 & 0 \\
400 & 0 \\
$>400$ & 0 \\
\hline
\end{tabular}

Histogram

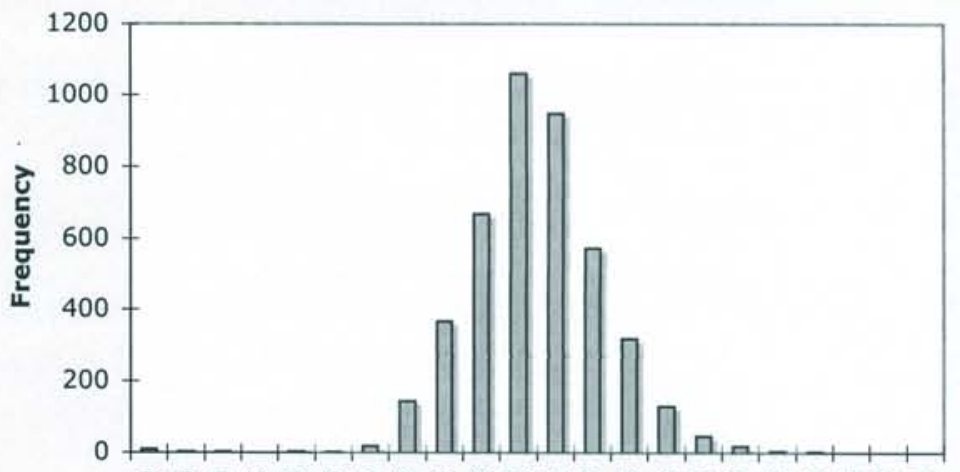

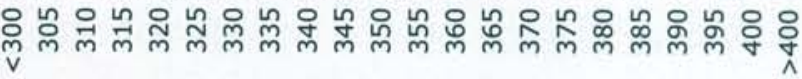

Mean Diameter $(\mu \mathrm{m})$

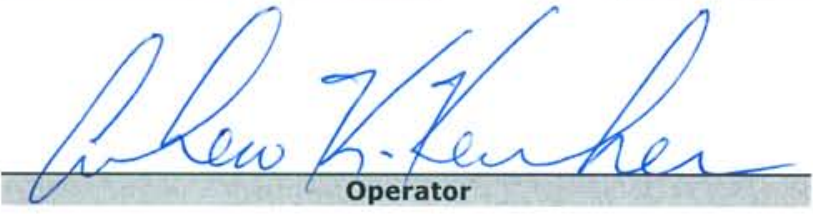

$$
\text { Play } 23,2005
$$


Data Report Form DRF-09B: Measurement of Kernel Ellipticity (Dmax/Dmin)

\begin{tabular}{|c|c|}
\hline Procedure: & AGR-CHAR-DAM-09 Rev. 0 \\
\hline Operator: & Andrew K. Kercher \\
\hline Folder name containing images: & IImc-agr|AGR\ImageProcessing|P05051902 \\
\hline Sample ID: & EEUEO350-01B renamed LEUO1-OIK-B \\
\hline Sample Description: & BWXT LEUCO kernel composite 69302 \\
\hline
\end{tabular}

Number of kernels analyzed: 4304

Number of kernels with ellipticity >1.05: 94

Average kernel ellipticity: 1.021

\begin{tabular}{|rr|r|r|}
\hline \multicolumn{2}{|c|}{ Distribution of the ellipticity (top binned) } \\
\hline Ellipticity $(D)$ & Frequency \\
\hline 1.005 & 10 \\
1.01 & 439 \\
1.015 & 1025 \\
1.02 & 1003 \\
1.025 & 754 \\
1.03 & 462 \\
1.035 & 218 \\
1.04 & 166 \\
1.045 & 89 \\
1.05 & 44 \\
1.055 & 29 \\
1.06 & 21 \\
1.065 & 17 \\
1.07 & 12 \\
1.075 & 5 \\
1.08 & 3 \\
1.085 & 1 \\
1.09 & 2 \\
1.095 & 0 \\
1.1 & 1 \\
$>1.1$ & 3
\end{tabular}
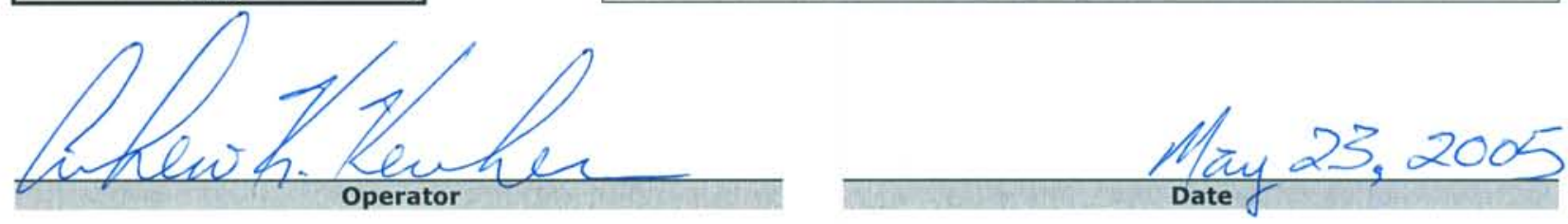
ORNL/TM-2006/019, Rev. 1

Data Report Form DRF-15: Measurement of Average Kernel Envelope Density using a Mercury Porosimeter

\begin{tabular}{|r|l|}
\hline Procedure: & AGR-CHAR-DAM-15 Rev. 2 \\
\hline Operator: & S. D. NUNN \\
\hline Kernel Lot ID: & LEUCO350-01 renamed LEJ OI-01 K \\
\hline Kernel Lot Description: & BWXT LEUCO KERNEL COMPOSITE 69302 \\
\hline Thermocouple Expiration Date: & $5 / 23 / 06$ \\
\hline Penetrometer Expiration Date: & $5 / 25 / 06$ \\
\hline Completed DRF Filename: & IImC-agr\AGRIPorosimeterIS05052701/S05052701R1_DRF15R2 \\
\hline
\end{tabular}

\begin{tabular}{|c|l|}
\hline Mean average weight/kernel $(\mathrm{g}):$ & $2.42 \mathrm{E}-04$ \\
\hline Uncertainty in mean average weight/kernel $(\mathrm{g}):$ & $5.96 \mathrm{E}-07$ \\
\hline
\end{tabular}

\begin{tabular}{|c|c|c|c|c|c|}
\hline & Sample 1 & Sample 2 & Sample 3 & Sample 4 & Sample 5 \\
\hline Porosimeter data file number: & S05052701L & S05052702L & S05052703L & S05052704L & S05052705L \\
\hline Weight of kernels $(\mathrm{g})$ : & 12.5822 & 12.5075 & 12.8773 & 12.6448 & 12.6895 \\
\hline Approximate number of kernels: & 52036 & 51727 & 53256 & 52294 & 52479 \\
\hline Uncertainty in number of kernels: & 128 & 128 & 131 & 129 & 129 \\
\hline Envelope volume of sample (cc): & 1.150 & 1.145 & 1.180 & 1.157 & 1.164 \\
\hline Average envelope volume/kernel (cc): & $2.21 \mathrm{E}-05$ & $2.21 \mathrm{E}-05$ & $2.22 \mathrm{E}-05$ & $2.21 \mathrm{E}-05$ & $2.22 \mathrm{E}-05$ \\
\hline Sample envelope density $(\mathrm{g} / \mathrm{cc})$ : & 10.943 & 10.925 & 10.912 & 10.932 & 10.906 \\
\hline
\end{tabular}

\begin{tabular}{|r|l|}
\hline Mean average envelope volume/kernel $(\mathrm{cc}):$ & $2.214 \mathrm{E}-05$ \\
\hline Uncertainty in mean envelope volume/kernel $(\mathrm{cc}):$ & $1.34 \mathrm{E}-08$ \\
\hline Mean sample envelope density $(\mathrm{g} / \mathrm{cc}):$ & 10.924 \\
\hline Standard deviation in sample envelope density $(\mathrm{g} / \mathrm{cc}):$ & 0.015 \\
\hline
\end{tabular}
S.D. D/unn
Operator
$\frac{2114106}{\text { Date }}$ 
Data Report Form DRF-22: Estimation of Average Particle Weight

$$
\begin{array}{r|l}
\text { Procedure: } & \text { AGR-CHAR-DAM-22 Rev. } 1 \\
\text { Operator: } & \text { John Hunn } \\
\hline
\end{array}
$$$$
\text { Particle Lot ID: LEU01-32K-A }
$$

Particle Lot Description: BWXT kernel composite 69302

Filename: \\mc-agr\AGR\ParticleWeight|W06020601_DRF22R1.xIs

\begin{tabular}{|r|c|c|c|c|c|}
\hline & Sample 1 & Sample 2 & Sample 3 & Sample 4 & Sample 5 \\
\hline Weight of particles (g): & $5.76 \mathrm{E}-02$ & $5.33 \mathrm{E}-02$ & $5.20 \mathrm{E}-02$ & $4.90 \mathrm{E}-02$ & $4.85 \mathrm{E}-02$ \\
\hline Number of particles: & 239 & 220 & 215 & 204 & 199 \\
\hline Average weight/particle (g): & $2.41 \mathrm{E}-04$ & $2.42 \mathrm{E}-04$ & $2.42 \mathrm{E}-04$ & $2.40 \mathrm{E}-04$ & $2.44 \mathrm{E}-04$ \\
\hline
\end{tabular}

Mean average weight/particle $(\mathrm{g}): 2.418 \mathrm{E}-04$

Uncertainty in mean average weight/particle $(g): 5.96 \mathrm{E}-07$

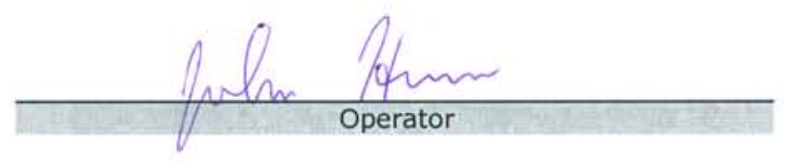

$$
\frac{2-6-06}{\text { Date }}
$$




\section{Characterization of NUCO kernel composite}

This section contains data on the kernel composite used for buffer and IPyC process qualification batches. The data was obtained according to product inspection plan AGR-CHAR-PIP-01R1. Some of the kernel data in this section was used as input for subsequent measurements of coating properties (e.g., buffer density). This is only a partial analysis of the kernel composite and was not used for product acceptance. Characterization of the kernel composite for acceptance according to the specific requirements listed in section 4 of INL EDF-4380, is documented in the BWXT data package for G73B-NU-69300. The BWXT kernel lot G73B-NU-69300 was riffled into sublots for characterization and coating. The ORNL identification for these kernels was NUCO350-\#\# (where \#\# were a series of integers beginning with 01).

Note that the average kernel weights were remeasured using a revised procedure which improved the accuracy of the reported value by improving the randomness of the measured samples. The NUCO average kernel weight changed from $(2.39 \pm 0.03) \cdot 10^{-4}$ grams per kernel to $(2.308 \pm 0.009) \cdot 10^{-4}$ grams per kernel. On data report form DRF-15 for the measurement of average kernel envelope density, the average kernel weight is used to approximate the number of kernels in a sample from the weight of the sample. The approximate number of kernels in a sample is divided into the sample envelope volume to determine the average kernel envelope volume. The change in average kernel weight resulted in a significant change in the calculated average kernel envelope volume. The data in this section shows the new average kernel weight and average kernel envelope volume. Records showing the original values are included in section 16.

The following pages shows the inspection report form (IRF-01). Following IRF-01 are the individual data report forms for the measurements that were performed. 
ORNL/TM-2006/019, Rev. 1

Inspection Report Form IRF-01: BWXT NUCO Kernel Composite 69300

Procedure: $\mid$ AGR-CHAR-PIP-01 Rev. 1

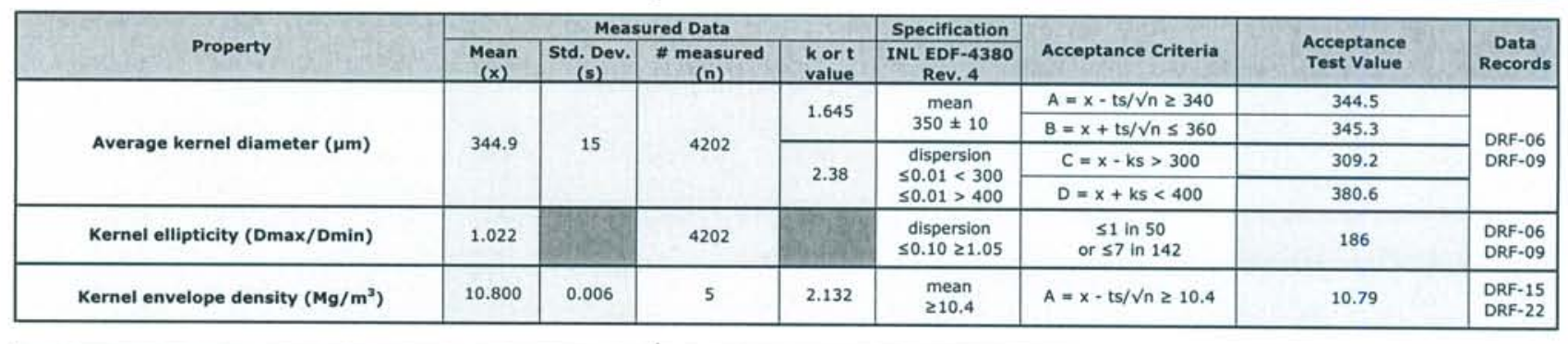

Comments

186 kernels with ellipticity $\geq 1.05$ out of 4202 kernels measured passes the dispersion specification acceptance criteria of $\leq 387$ in 4202 .

This composite would pass an ellipticity control limit of $\geq 1.038$ at $10 \%$ tolerance limit with $95 \%$ confidence level.
This composite would pass an ellipticity control limit of $\geq 1.05$ at $5 \%$ tolerance limit with $95 \%$ confidence level.
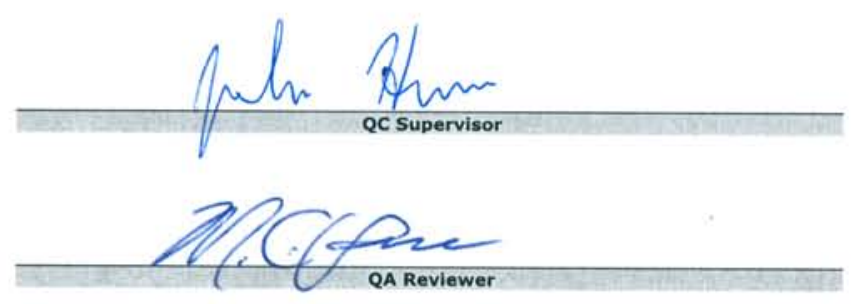

$2-20-06$

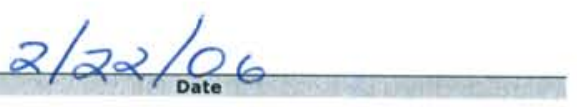

47 
Data Report Form DRF-06: Imaging of Kernel Diameter and Ellipticity Using an Optical Microscope System

Procedure: AGR-CHAR-DAM-06 Rev. 0

Operator: Andrew Nelson

Sample ID: NUCO350-26B

Sample Description: 350 um NUCO kernels from BWXT 69300 Composite

Folder name containing images: \Imc-agr\AGR\ImageProcessing \P05052501

DMR Calibration Expiration Date: 3/25/06

Stage Micrometer Calibration Expiration Date: $2 / 17 / 07$

Measured Value for $760 \mu \mathrm{m}$ in Stage Micrometer Image: $760.6 \mu \mathrm{m}$

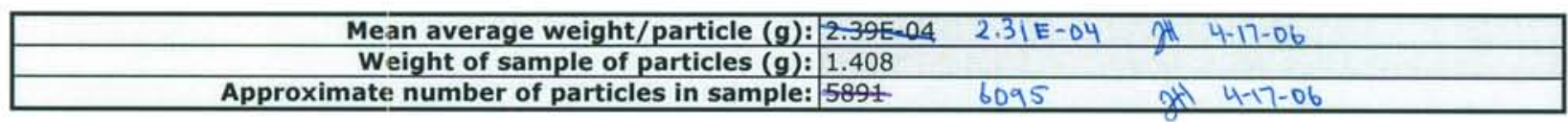
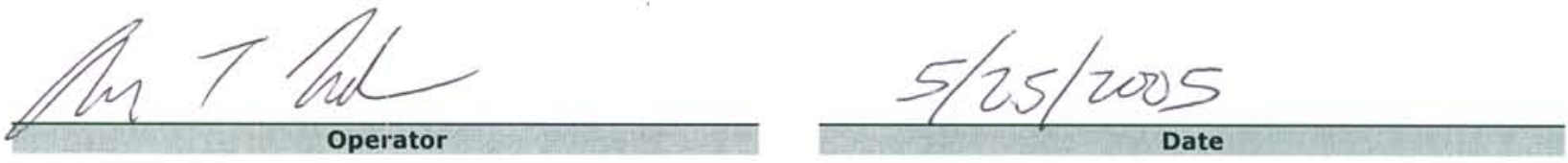
Data Report Form DRF-09A: Measurement of Kernel Diameter

\begin{tabular}{|c|c|}
\hline Procedure: & AGR-CHAR-DAM-09 Rev. 0 \\
\hline Operator: & Andrew K. Kercher \\
\hline Folder name containing images: & IImc-agr|AGR\ImageProcessing\P05052501 \\
\hline $\begin{array}{r}\text { Sample ID: } \\
\text { Sample Description: }\end{array}$ & NUCO350-26B NUCO kernels \\
\hline Folder name containing processed data: & 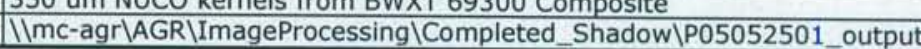 \\
\hline
\end{tabular}

Number of kernels analyzed: 4202

Mean of the average diameter of each kernel $(\mu \mathrm{m}): 344.9$

Standard deviation in the average diameter of each kernel $(\mu \mathrm{m}): 15$

Distribution of the average kernel diameter (top binned)

\begin{tabular}{|rr|}
\hline Mean Diameter & Frequency \\
\hline$<300$ & 36 \\
305 & 1 \\
310 & 11 \\
315 & 15 \\
320 & 61 \\
325 & 156 \\
330 & 204 \\
335 & 233 \\
340 & 412 \\
345 & 867 \\
350 & 858 \\
355 & 603 \\
360 & 383 \\
365 & 182 \\
370 & 71 \\
375 & 48 \\
380 & 16 \\
385 & 8 \\
390 & 7 \\
395 & 5 \\
400 & 3 \\
$>400$ & 22 \\
\hline
\end{tabular}
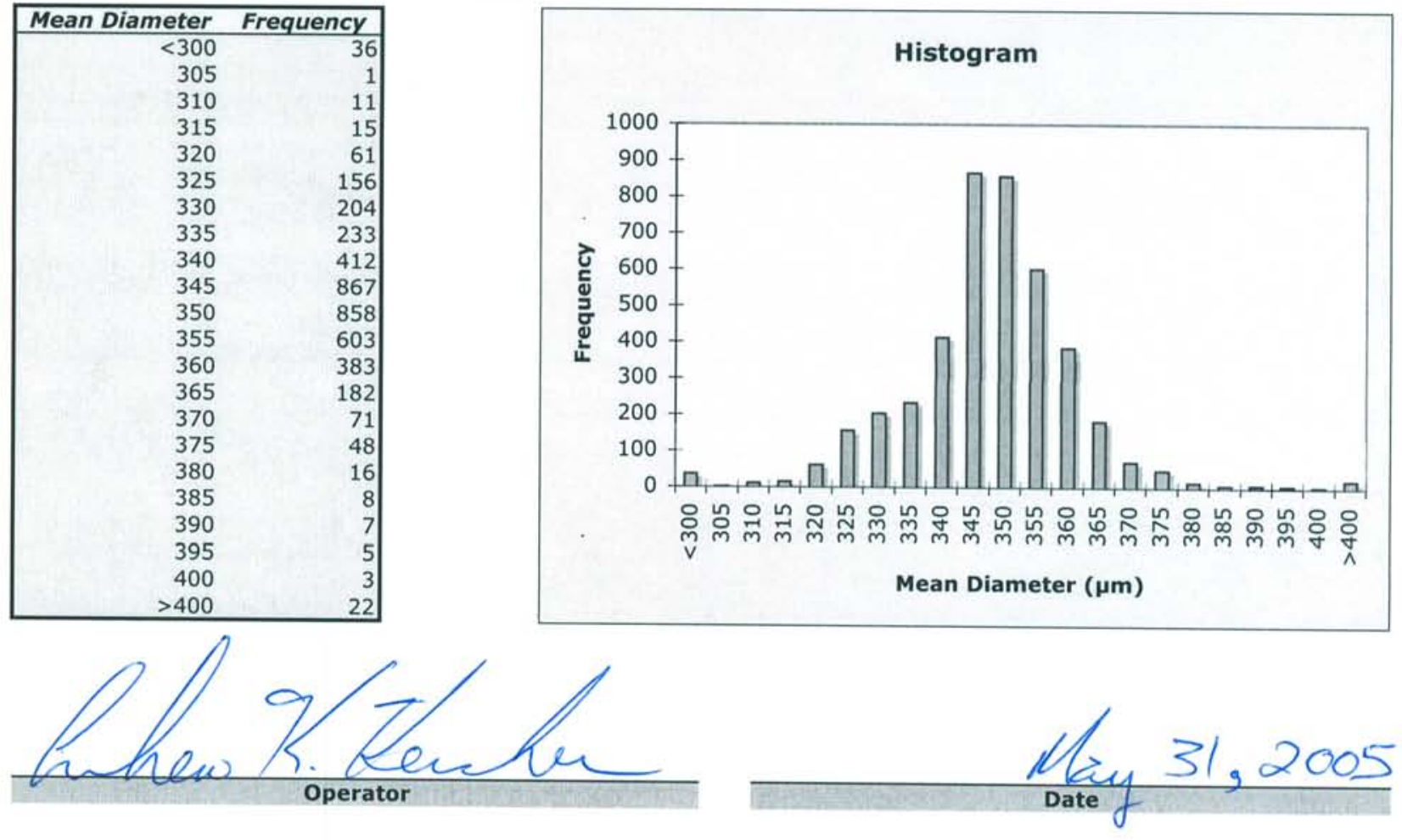
Data Report Form DRF-09B: Measurement of Kernel Ellipticity (Dmax/Dmin)

Procedure: AGR-CHAR-DAM-09 Rev. 0

Operator: Andrew K. Kercher

Folder name containing images: |lmc-agr|AGR|ImageProcessing|P05052501

Sample ID: NUCO350-26B NUCO kernels

Sample Description: 350 um NUCO kernels from BWXT 69300 Composite

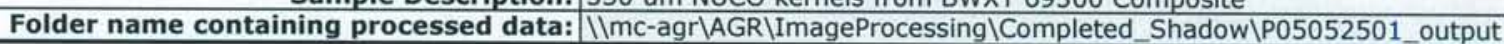

Number of kernels analyzed: 4202

Number of kernels with ellipticity >1.05: 186

Average kernel ellipticity: 1.022

\section{Distribution of the ellipticity (top binned)}

\begin{tabular}{|rr|}
\hline Ellipticity $(\boldsymbol{D})$ & Frequency \\
\hline 1.005 & 15 \\
1.010 & 486 \\
1.015 & 1069 \\
1.020 & 979 \\
1.025 & 627 \\
1.030 & 361 \\
1.035 & 197 \\
1.040 & 141 \\
1.045 & 89 \\
1.050 & 52 \\
1.055 & 42 \\
1.060 & 19 \\
1.065 & 17 \\
1.070 & 19 \\
1.075 & 11 \\
1.080 & 12 \\
1.085 & 15 \\
1.090 & 8 \\
1.095 & 11 \\
1.100 & 2 \\
$>1.100$ & 30 \\
\hline
\end{tabular}
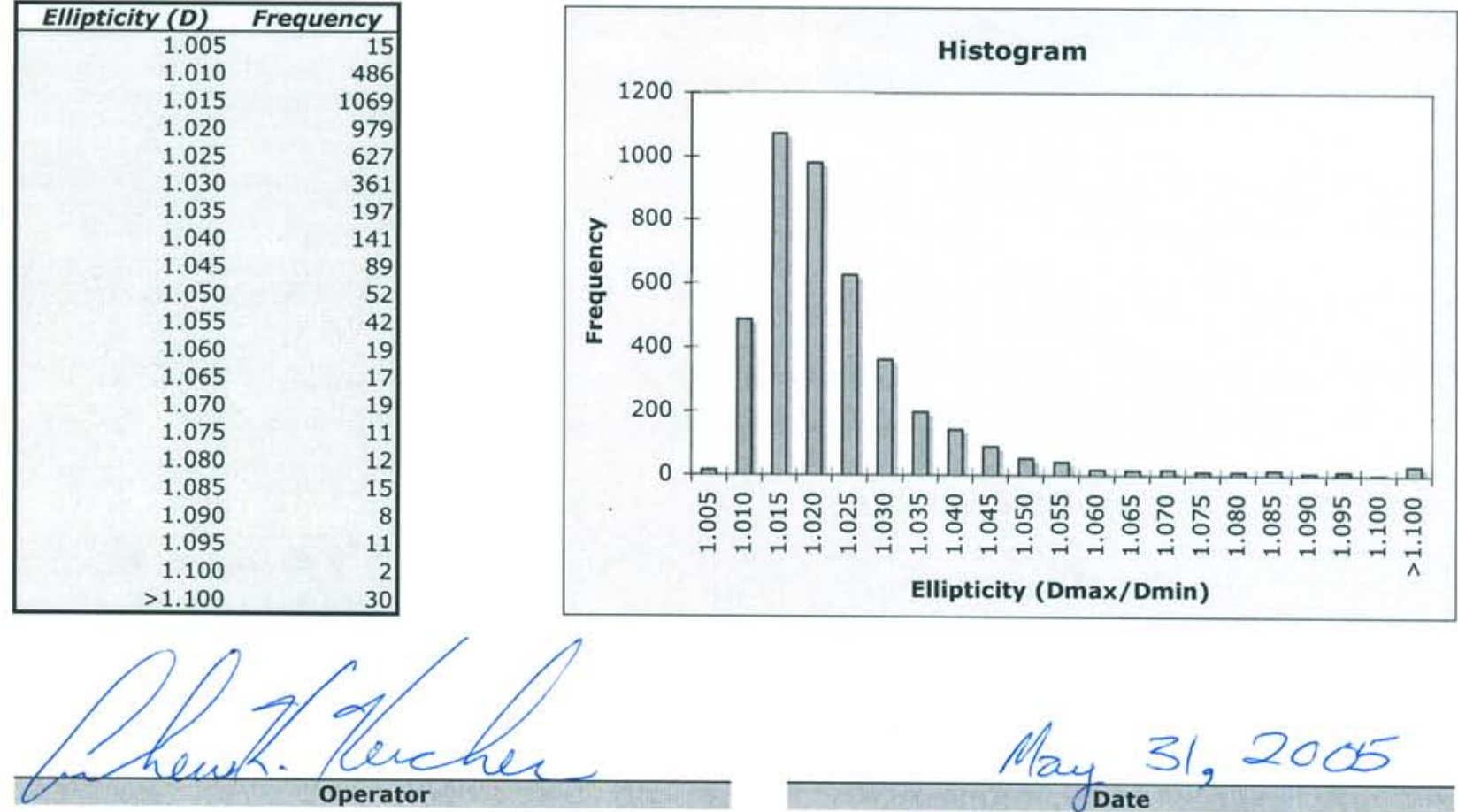

$$
\text { May } 31,2005
$$

Date 
Data Report Form DRF-22: Estimation of Average Particle Weight

\begin{tabular}{|r|l|}
\hline Procedure: & AGR-CHAR-DAM-22 Rev. 1 \\
\hline Operator: & John Hunn \\
\hline Particle Lot ID: & NUCO350-42-A \\
\hline Particle Lot Description: & BWXT kernel composite 69300 \\
\hline Filename: & IImC-agr\AGR\ParticleWeight\W06020302_DRF22R1.xIs \\
\hline
\end{tabular}

\begin{tabular}{|r|c|c|c|c|c|}
\hline & Sample 1 & Sample 2 & Sample 3 & Sample 4 & Sample 5 \\
\hline Weight of particles (g): & $3.13 \mathrm{E}-02$ & $2.84 \mathrm{E}-02$ & $3.40 \mathrm{E}-02$ & $3.48 \mathrm{E}-02$ & $3.40 \mathrm{E}-02$ \\
\hline Number of particles: & 136 & 123 & 149 & 149 & 147 \\
\hline Average weight/particle (g): & $2.30 \mathrm{E}-04$ & $2.31 \mathrm{E}-04$ & $2.28 \mathrm{E}-04$ & $2.34 \mathrm{E}-04$ & $2.31 \mathrm{E}-04$ \\
\hline
\end{tabular}

Mean average weight/particle $(\mathrm{g}): 2.308 \mathrm{E}-04$

Uncertainty in mean average weight/particle $(g): 0.69 \mathrm{E}-07$

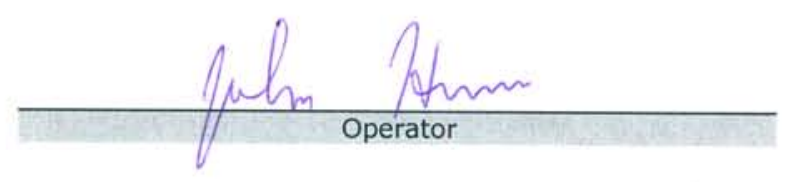

$$
\frac{2-3-0 b}{\text { Date }}
$$




\section{Characterization of buffer on interrupted batches}

This section contains data on the buffer coating density and other buffer properties for particles removed from the coater after deposition of only the buffer layer. The data was obtained according to product inspection plan AGR-CHAR-PIP-02R4. The buffer on these particles was deposited under similar conditions as the buffer on the particles in composite LEU01-46T and should therefore be representative of the buffer on the particles in that composite. Density measurements were made on these interrupted batches because it was not feasible to measure the buffer density after all coating layers were applied. According to section 5.3 of EDF-4380, Rev. 6 , three representative buffer layers from interrupted batches which meet the specification for buffer density are sufficient to qualify the process for buffer density. The qualifying batches used $350 \mu \mathrm{m}$ natural uranium oxide/uranium carbide kernels (NUCO). The kernels were obtained from BWXT and were identified as composite G73B-NU-69300. The use of NUCO surrogate kernels was not expected to significantly effect the density of the buffer. A confirmatory batch using LEUCO kernels from G73D-20-69302 was coated and characterized to verify this assumption.

Note that the average kernel weights and volumes for both the LEUCO and NUCO kernel composites were remeasured after coated particle composite characterization was underway in order to improve the accuracy of the reported value, as discussed in sections 6 and 7. On data report form DRF-16 for the buffer envelope density, the average kernel weight and volume are used to calculate the buffer density. The change in average kernel weight and volume resulted in a significant change in the calculated buffer densities previously used to determine acceptability of the buffer deposition conditions. The data in this section shows the new average kernel weight, average kernel volume, and buffer densities. Records showing the original values are included in section 16. The new values for buffer density were about $10 \%$ higher than the originally reported values, but both were within the range specified in INL EDF-4380, Rev. 6.

The following page shows the inspection report form (IRF-02A). Following IRF-02A are the individual data report forms for the measurements that were performed. Additional data at the end of this section is provided for information only. The process conditions used to deposit the buffer layer were determined to be sufficient to satisfy the specifications in section 5.3 of EDF 4380, Rev. 6. 


\begin{tabular}{|r|l|}
\hline \multicolumn{1}{|c|}{ Procedure: } & AGR-CHAR-PIP-02 Rev. 4 \\
\hline Batch 1 ID: & NUCO350-25B \\
\hline Batch 1 description: & Buffer-Coated BWXT kernel composite 69300 \\
\hline Batch 2 ID: & NUCO350-36B \\
\hline Batch 2 description: & Buffer-Coated BWXT kernel composite 69300 \\
\hline Batch 3 ID: & NUCO350-54B \\
\hline Batch 3 description: & Buffer-Coated BWXT kernel composite 69300 \\
\hline
\end{tabular}

\begin{tabular}{|c|c|c|c|c|c|c|c|c|c|}
\hline \multirow[b]{2}{*}{ Property } & \multicolumn{4}{|c|}{ Measured Data } & \multirow{2}{*}{$\begin{array}{c}\text { Specification } \\
\text { INL EDF-4380 } \\
\text { Rev. } 6\end{array}$} & \multirow[b]{2}{*}{ Acceptance Criteria } & \multirow{2}{*}{$\begin{array}{l}\text { Acceptance } \\
\text { Test Value }\end{array}$} & \multirow{2}{*}{$\begin{array}{l}\text { Pass } \\
\text { or } \\
\text { fail }\end{array}$} & \multirow{2}{*}{$\begin{array}{c}\text { Data } \\
\text { Records }\end{array}$} \\
\hline & $\begin{array}{c}\text { Mean } \\
(x)\end{array}$ & $\begin{array}{c}\text { Std. Dev. } \\
(\mathrm{s})\end{array}$ & $\begin{array}{l}\text { \# measured } \\
(\mathrm{n})\end{array}$ & $\begin{array}{l}t \text { value } \\
(t)\end{array}$ & & & & & \\
\hline \multirow{2}{*}{ Batch 1: Buffer envelope density $\left(\mathrm{Mg} / \mathrm{m}^{3}\right)$} & \multirow{2}{*}{1.08} & \multirow{2}{*}{0.04} & \multirow{2}{*}{5} & \multirow{2}{*}{2.132} & \multirow{2}{*}{$\begin{array}{c}\text { mean } \\
1.03 \pm 0.15\end{array}$} & $A=x-t s / \sqrt{n} \geq 0.88$ & 1.04 & pass & \multirow{2}{*}{$\begin{array}{l}\text { DRF-16 } \\
\text { DRF-22 }\end{array}$} \\
\hline & & & & & & $B=x+t s / \sqrt{n} \leq 1.18$ & 1.12 & pass & \\
\hline \multirow{2}{*}{ Batch 2: Buffer envelope density $\left(\mathrm{Mg} / \mathrm{m}^{3}\right)$} & \multirow{2}{*}{1.11} & \multirow{2}{*}{0.05} & \multirow{2}{*}{5} & \multirow{2}{*}{2.132} & \multirow{2}{*}{$\begin{array}{c}\text { mean } \\
1.03 \pm 0.15\end{array}$} & $A=x-t s / \sqrt{ } n \geq 0.88$ & 1.06 & pass & \multirow{2}{*}{$\begin{array}{l}\text { DRF-16 } \\
\text { DRF-22 }\end{array}$} \\
\hline & & & & & & $B=x+t s / \sqrt{n} \leq 1.18$ & 1.16 & pass & \\
\hline \multirow{2}{*}{ Batch 3: Buffer envelope density $\left(\mathrm{Mg} / \mathrm{m}^{3}\right)$} & \multirow{2}{*}{1.11} & \multirow{2}{*}{0.04} & \multirow{2}{*}{5} & \multirow{2}{*}{2.132} & \multirow{2}{*}{$\begin{array}{c}\text { mean } \\
1.03 \pm 0.15\end{array}$} & $A=x-t s / \sqrt{ } n \geq 0.88$ & 1.07 & pass & \multirow{2}{*}{$\begin{array}{l}\text { DRF-16 } \\
\text { DRF-22 }\end{array}$} \\
\hline & & & & & & $B=x+t s / \sqrt{n} \leq 1.18$ & 1.15 & pass & \\
\hline
\end{tabular}

Comments

Standard deviations are $\sqrt{5}$ times the uncertainties in buffer density (standard errors) reported on DRF-16.

Average thickness of buffer was $108 \mu \mathrm{m}$ based on average envelope volume of $9.25 \mathrm{E}-5 \mathrm{cc}$ (effective diameter of $561 \mu \mathrm{m}$ ) and average kernel diameter of $345 \mu \mathrm{m}$. Average thickness of buffer was $108 \mu \mathrm{m}$ based on average outer diameter of $561 \mu \mathrm{m}$ obtained per DAM-10 and average kernel diameter of $345 \mu \mathrm{m}$.

Confirmatory batch on LEUCO kernels, LEU01-163: mean buffer density $=1.10 \mathrm{~g} / \mathrm{cC}$.
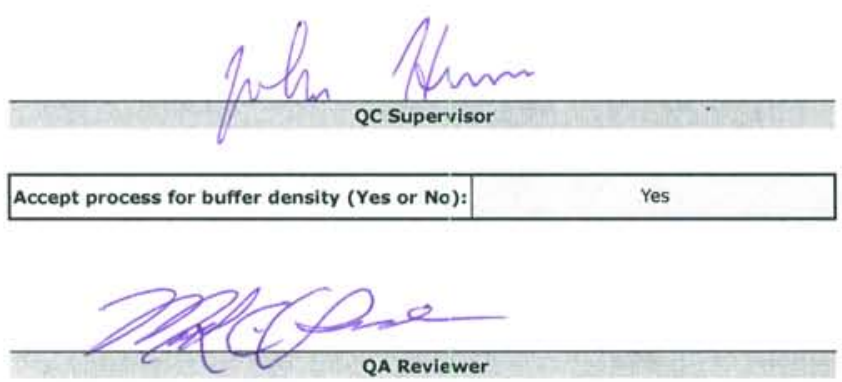

$$
3-10-06
$$

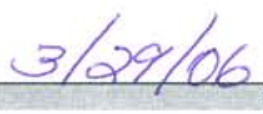


ORNL/TM-2006/019, Rev. 1

Data Report Form DRF-16: Measurement of Buffer Envelope Density using a Mercury Porosimeter

\begin{tabular}{|c|c|}
\hline Procedure: & AGR-CHAR-DAM-16 Rev. 2 \\
\hline Operator: & S. D. NUNN \\
\hline Buffer-coated kernel batch ID: & NUCO350-25B \\
\hline Batch Description: & Buffer Coated BWXT Kernel Composite 69300 \\
\hline Thermocouple Expiration Date: & $5 / 23 / 06$ \\
\hline Penetrometer Expiration Date: & $5 / 25 / 06$ \\
\hline Completed DRF Filename: & IImc-agr|AGRIPorosimeter\S05062001/S05062001R1_DRF16R2.xls \\
\hline
\end{tabular}

\begin{tabular}{|r|l|}
\hline Mean average weight/buffer-coated kernel $(g):$ & $3.07 \mathrm{E}-04$ \\
\hline Uncertainty in mean average weight/b-c kernel $(g):$ & $1.00 \mathrm{E}-06$ \\
\hline Mean average weight/bare kernel $(g):$ & $2.31 \mathrm{E}-04$ \\
\hline Uncertainty in mean average weight/bare kernel $(g):$ & $8.69 \mathrm{E}-07$ \\
\hline Mean average envelope volume/bare kernel $(\mathrm{cc}):$ & $2.14 \mathrm{E}-05$ \\
\hline Uncertainty in envelope volume/bare kernel $(\mathrm{cc}):$ & $5.47 \mathrm{E}-09$ \\
\hline
\end{tabular}

\begin{tabular}{|r|c|c|c|c|c|}
\cline { 2 - 5 } \multicolumn{1}{c|}{} & Sample 1 & Sample 2 & Sample 3 & Sample 4 & Sample 5 \\
\hline Porosimeter data file number: & S05062001L & S05062002L & S05062003L & S05062004L & S05062005L \\
\hline Weight of buffer-coated kernels $(g):$ & 4.1223 & 4.2165 & 4.4333 & 4.4104 & 4.0445 \\
\hline Approximate number of b-c kernels: & 13428 & 13735 & 14441 & 14366 & 13174 \\
\hline Uncertainty in number of b-c kernels: & 44 & 45 & 47 & 47 & 1.321 \\
\hline Total envelope volume of sample (cc): & 1.235 & 1.268 & 1.335 & 1.212 \\
\hline Av. envelope volume/b-c kernels (cc): & $9.19 \mathrm{E}-05$ & $9.23 \mathrm{E}-05$ & $9.24 \mathrm{E}-05$ & $9.19 \mathrm{E}-05$ & $9.20 \mathrm{E}-05$ \\
\hline Sample envelope density $(\mathrm{g} / \mathrm{cc}):$ & 3.339 & 3.326 & 3.321 & 3.340 & 3.338 \\
\hline
\end{tabular}

Mean average envelope volume/b-c kernel (cc): $9.21 \mathrm{E}-05$

Uncertainty in envelope volume/b-c kernel (cc): $1.1 \mathrm{E}-07$

Buffer density: $1.08 \mathrm{E}+00$

Uncertainty in buffer density: $1.88 \mathrm{E}-02$

S.D.MUinn Operator
$2 / 14 / 06$ Date 
ORNL/TM-2006/019, Rev. 1

Data Report Form DRF-22: Estimation of Average Particle Weight

\begin{tabular}{|r|l|}
\hline Procedure: & AGR-CHAR-DAM-22 Rev. 1 \\
\hline Operator: & Dixie Barker \\
\hline Particle Lot ID: & NUC0350-25B \\
\hline Particle Lot Description: & Buffer Coated NUCO \\
\hline Filename: & IImC-agr\AGR\ParticleWeight\W05062001_DRF22R1.xIs \\
\hline
\end{tabular}

\begin{tabular}{|r|c|c|c|c|c|}
\hline & Sample 1 & Sample 2 & Sample 3 & Sample 4 & Sample 5 \\
\hline Weight of particles (g): & $3.28 \mathrm{E}-02$ & $2.73 \mathrm{E}-02$ & $3.08 \mathrm{E}-02$ & $3.46 \mathrm{E}-02$ & $3.99 \mathrm{E}-02$ \\
\hline Number of particles: & 107 & 90 & 100 & 113 & 129 \\
\hline Average weight/particle (g): & $3.07 \mathrm{E}-04$ & $3.03 \mathrm{E}-04$ & $3.08 \mathrm{E}-04$ & $3.06 \mathrm{E}-04$ & $3.09 \mathrm{E}-04$ \\
\hline
\end{tabular}

Mean average weight/particle $(\mathrm{g}): 3.07 \mathrm{E}-04$ Uncertainty in mean average weight/particle $(\mathrm{g}): 1.00 \mathrm{E}-06$
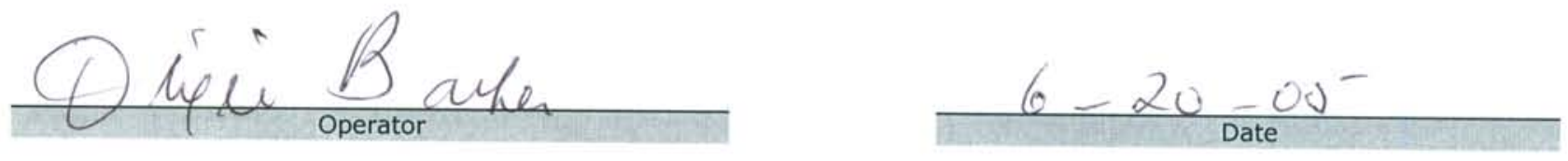

56 
ORNL/TM-2006/019, Rev. 1

Data Report Form DRF-16: Measurement of Buffer Envelope Density using a Mercury Porosimeter

\begin{tabular}{|r|l|}
\hline Procedure: & AGR-CHAR-DAM-16 Rev. 2 \\
\hline Operator: & S. D.NUNN \\
\hline Buffer-coated kernel batch ID: & NUCO350-36B \\
\hline Batch Description: & Buffer Coated BWXT Kernel Composite 69300 \\
\hline Thermocouple Expiration Date: & $5 / 23 / 06$ \\
\hline Penetrometer Expiration Date: & $5 / 25 / 06$ \\
\hline Completed DRF Filename: & IImc-agrVAGR\Porosimeter\S05062006IS05062006R1_DRF16R2.xIs \\
\hline
\end{tabular}

\begin{tabular}{|r|l|}
\hline Mean average weight/buffer-coated kernel $(g):$ & $3.11 \mathrm{E}-04$ \\
\hline Uncertainty in mean average weight/b-c kernel $(g):$ & $1.20 \mathrm{E}-06$ \\
\hline Mean average weight/bare kernel $(g):$ & $2.31 \mathrm{E}-04$ \\
\hline Uncertainty in mean average weight/bare kernel $(g):$ & $8.69 \mathrm{E}-07$ \\
\hline Mean average envelope volume/bare kernel $(\mathrm{cc}):$ & $2.14 \mathrm{E}-05$ \\
\hline Uncertainty in envelope volume/bare kernel $(\mathrm{cc}):$ & $5.47 \mathrm{E}-09$ \\
\hline
\end{tabular}

\begin{tabular}{|r|c|c|c|c|c|}
\cline { 2 - 5 } \multicolumn{1}{c|}{} & Sample 1 & Sample 2 & Sample 3 & Sample 4 & Sample 5 \\
\hline Porosimeter data file number: & S05062006L & S05062007L & S05062008L & S05062101L & S05062102L \\
\hline Weight of buffer-coated kernels $(\mathrm{g}):$ & 4.0723 & 4.0655 & 4.0860 & 4.0696 & 4.0514 \\
\hline Approximate number of b-c kernels: & 13094 & 13072 & 13138 & 13086 & 13027 \\
\hline Uncertainty in number of b-c kernels: & 51 & 50 & 51 & 50 & 50 \\
\hline Total envelope volume of sample (cc): & 1.226 & 1.227 & 1.232 & 1.226 & 1.217 \\
\hline Av. envelope volume/b-c kernels (cc): & $9.37 \mathrm{E}-05$ & $9.38 \mathrm{E}-05$ & $9.38 \mathrm{E}-05$ & $9.37 \mathrm{E}-05$ & $9.34 \mathrm{E}-05$ \\
\hline Sample envelope density $(\mathrm{g} / \mathrm{cc}):$ & 3.321 & 3.315 & 3.317 & 3.319 & 3.328 \\
\hline
\end{tabular}

Mean average envelope volume/b-c kernel (cc): $: 9.37 \mathrm{E}-05$

Uncertainty in envelope volume/b-c kernel (cc): $6.7 \mathrm{E}-08$

Buffer density: $1.11 \mathrm{E}+00$

Uncertainty in buffer density: $2.05 \mathrm{E}-02$

S.D. ) lum n

Operator $\frac{2114106}{\text { Date }}$ 
Data Report Form DRF-22: Estimation of Average Particle Weight

\begin{tabular}{|r|l|}
\hline Procedure: & AGR-CHAR-DAM-22 Rev. 1 \\
\hline Operator: & Dixie Barker \\
\hline Particle Lot ID: & NUCO350-36B \\
\hline Particle Lot Description: & Buffer Coated NUCO \\
\hline Filename: & IImC-agr\AGR\ParticleWeight\W05062002_DRF22R1.xIs \\
\hline
\end{tabular}

\begin{tabular}{|r|c|c|c|c|c|}
\hline & Sample 1 & Sample 2 & Sample 3 & Sample 4 & Sample 5 \\
\hline Weight of particles (g): & $3.45 \mathrm{E}-02$ & $3.26 \mathrm{E}-02$ & $3.54 \mathrm{E}-02$ & $3.58 \mathrm{E}-02$ & $3.42 \mathrm{E}-02$ \\
\hline Number of particles: & 110 & 104 & 115 & 116 & 110 \\
\hline Average weight/particle (g): & $3.14 \mathrm{E}-04$ & $3.13 \mathrm{E}-04$ & $3.08 \mathrm{E}-04$ & $3.09 \mathrm{E}-04$ & $3.11 \mathrm{E}-04$ \\
\hline
\end{tabular}

Mean average weight/particle $(\mathrm{g}): 3.11 \mathrm{E}-04$

Uncertainty in mean average weight/particle $(g): 1.20 \mathrm{E}-06$
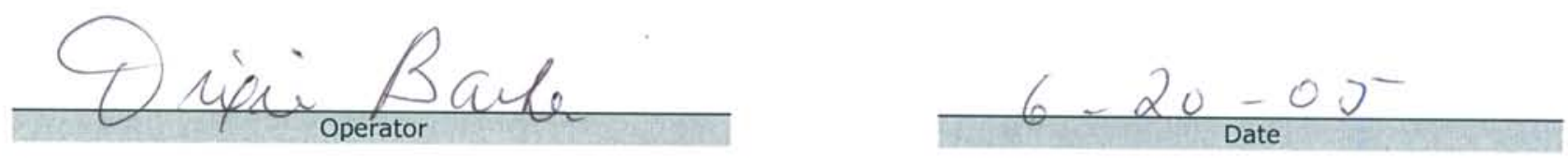
ORNL/TM-2006/019, Rev. 1

Data Report Form DRF-16: Measurement of Buffer Envelope Density using a Mercury Porosimeter

\begin{tabular}{|c|c|}
\hline Procedure: & AGR-CHAR-DAM-16 Rev. 2 \\
\hline Operator: & S. D.NUNN \\
\hline Buffer-coated kernel batch ID: & NUCO350-54B \\
\hline Batch Description: & Buffer Coated BWXT Kernel Composite 69300 \\
\hline Thermocouple Expiration Date: & $5 / 23 / 06$ \\
\hline Penetrometer Expiration Date: & $5 / 25 / 06$ \\
\hline Completed DRF Filename: & 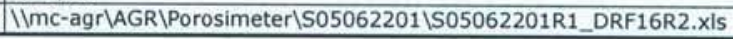 \\
\hline
\end{tabular}

\begin{tabular}{|r|l|}
\hline Mean average weight/buffer-coated kernel $(g):$ & $3.11 \mathrm{E}-04$ \\
\hline Uncertainty in mean average weight/b-c kernel $(g):$ & $1.09 \mathrm{E}-06$ \\
\hline Mean average weight/bare kernel $(g):$ & $2.31 \mathrm{E}-04$ \\
\hline Uncertainty in mean average weight/bare kernel $(g):$ & $8.69 \mathrm{E}-07$ \\
\hline Mean average envelope volume/bare kernel $(\mathrm{cc}):$ & $2.14 \mathrm{E}-05$ \\
\hline Uncertainty in envelope volume/bare kernel $(\mathrm{cc}):$ & $5.47 \mathrm{E}-09$ \\
\hline
\end{tabular}

\begin{tabular}{|r|c|c|c|c|c|}
\cline { 2 - 5 } \multicolumn{1}{c|}{} & Sample 1 & Sample 2 & Sample 3 & Sample 4 & Sample 5 \\
\hline Porosimeter data file number: & S05062201L & S05062202L & S05062203L & S05062204L & S05062205L \\
\hline Weight of buffer-coated kernels (g): & 4.1223 & 4.0694 & 4.0160 & 4.0391 & 4.0471 \\
\hline Approximate number of b-c kernels: & 13255 & 13085 & 12913 & 12987 & 13013 \\
\hline Uncertainty in number of b-c kernels: & 46 & 46 & 45 & 46 & 1.215 \\
\hline Total envelope volume of sample (cc): & 1.233 & 1.225 & 1.209 & 1.215 \\
\hline Av. envelope volume/b-c kernels (cc): & $9.30 \mathrm{E}-05$ & $9.36 \mathrm{E}-05$ & $9.36 \mathrm{E}-05$ & $9.35 \mathrm{E}-05$ & $9.34 \mathrm{E}-05$ \\
\hline Sample envelope density $(\mathrm{g} / \mathrm{cc}):$ & 3.344 & 3.323 & 3.323 & 3.326 & 3.331 \\
\hline
\end{tabular}

Mean average envelope volume/b-c kernel (cc): $9.34 \mathrm{E}-05$

Uncertainty in envelope volume/b-c kernel (cc): $1.1 \mathrm{E}-07$

Buffer density: $1.11 \mathrm{E}+00$

Uncertainty in buffer density: $1.94 \mathrm{E}-02$

S.D. D) $1 \ln ^{2}$ Operator $\frac{2114106}{\text { Date }}$ 
ORNL/TM-2006/019, Rev. 1

Data Report Form DRF-22: Estimation of Average Particle Weight

Procedure: AGR-CHAR-DAM-22 Rev. 1 Particle Lot ID: NUCO350-54

Particle Lot Description: Buffer Coated NUCO

Filename: IImc-agr|AGR\ParticleWeight|W05062101_DRF22R1.xls

\begin{tabular}{l}
\begin{tabular}{r|c|c|c|c|c|}
\hline & Sample 1 & Sample 2 & Sample 3 & Sample 4 & Sample 5 \\
\hline Weight of particles (g): & $3.66 \mathrm{E}-02$ & $4.39 \mathrm{E}-02$ & $3.80 \mathrm{E}-02$ & $4.22 \mathrm{E}-02$ & $3.97 \mathrm{E}-02$ \\
\hline Number of particles: & 118 & 140 & 123 & 135 & 129 \\
\hline Average weight/particle (g): & $3.10 \mathrm{E}-04$ & $3.14 \mathrm{E}-04$ & $3.09 \mathrm{E}-04$ & $3.13 \mathrm{E}-04$ & $3.08 \mathrm{E}-04$ \\
\hline
\end{tabular} \\
\begin{aligned} & \hline Mean average weight/particle (g): $3.11 \mathrm{E}-04 \\
&$\hline\end{aligned} \\
Uncertainty in mean average weight/particle (g): $1.09 \mathrm{E}-06$ \\
\hline
\end{tabular}

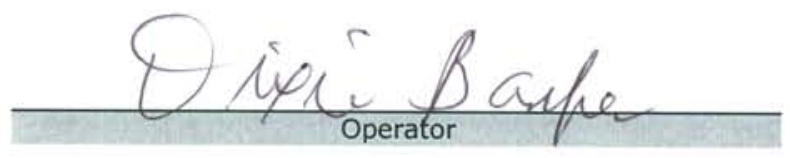

$$
\frac{6-21-05}{\text { Date }}
$$

60 


\section{For Information Only}

The information in the remainder of this section reports results of measurements not required by the fuel specification and is provided for information only. 
ORNL/TM-2006/019, Rev. 1

Data Report Form DRF-16: Measurement of Buffer Envelope Density using a Mercury Porosimeter

\begin{tabular}{|c|c|}
\hline Procedure: & AGR-CHAR-DAM-16 Rev. 2 \\
\hline Operator: & S. D.NUNN \\
\hline Buffer-coated kernel batch ID: & NUCO350-58B \\
\hline Batch Description: & Composite of Buffer Coated BWXT Kernel Composite 69300 \\
\hline Thermocouple Expiration Date: & $5 / 23 / 06$ \\
\hline Penetrometer Expiration Date: & $5 / 25 / 06$ \\
\hline Completed DRF Filename: & 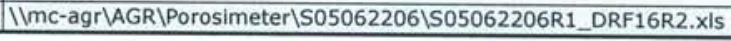 \\
\hline
\end{tabular}

\begin{tabular}{|r|l|}
\hline Mean average weight/buffer-coated kernel $(g):$ & $3.09 \mathrm{E}-04$ \\
\hline Uncertainty in mean average weight/b-c kernel $(g):$ & $4.99 \mathrm{E}-07$ \\
\hline Mean average weight/bare kernel $(g):$ & $2.31 \mathrm{E}-04$ \\
\hline Uncertainty in mean average weight/bare kernel $(g):$ & $8.69 \mathrm{E}-07$ \\
\hline Mean average envelope volume/bare kernel $(\mathrm{cc}):$ & $2.14 \mathrm{E}-05$ \\
\hline Uncertainty in envelope volume/bare kernel $(\mathrm{cc}):$ & $5.47 \mathrm{E}-09$ \\
\hline
\end{tabular}

\begin{tabular}{|r|c|c|c|c|c|}
\cline { 2 - 5 } \multicolumn{1}{c|}{} & Sample 1 & Sample 2 & Sample 3 & Sample 4 & Sample 5 \\
\hline Porosimeter data file number: & S05062206L & S05062207L & S05062301L & S05062302L & S05062303L \\
\hline Weight of buffer-coated kernels $(\mathrm{g}):$ & 4.3399 & 4.3264 & 4.3459 & 4.4478 & 4.3485 \\
\hline Approximate number of b-c kernels: & 14045 & 14001 & 14064 & 14394 & 14073 \\
\hline Uncertainty in number of b-c kernels: & 23 & 23 & 23 & 23 & 1.33 \\
\hline Total envelope volume of sample (cc): & 1.300 & 1.296 & 1.300 & 1.333 \\
\hline Av. envelope volume/b-c kernels (cc): & $9.25 \mathrm{E}-05$ & $9.26 \mathrm{E}-05$ & $9.24 \mathrm{E}-05$ & $9.26 \mathrm{E}-05$ & $9.26 \mathrm{E}-05$ \\
\hline Sample envelope density $(\mathrm{g} / \mathrm{cc}):$ & 3.340 & 3.338 & 3.344 & 3.337 & 3.338 \\
\hline
\end{tabular}

Mean average envelope volume/b-c kernel (cc): $19.25 \mathrm{E}-05$

Uncertainty in envelope volume/b-c kernel (cc): $3.8 \mathrm{E}-08$

Buffer density: $1.10 \mathrm{E}+00$

Uncertainty in buffer density: $1.41 \mathrm{E}-02$

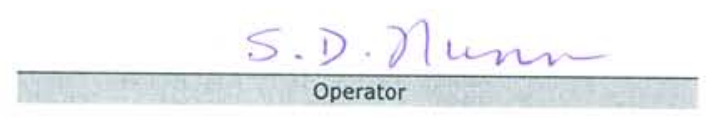

$\frac{211+106}{\text { Date }}$ 
Data Report Form DRF-22: Estimation of Average Particle Weight

Procedure: AGR-CHAR-DAM-22 Rev. 1

Operator: Dixie Barker

Particle Lot ID: NUCO350-58B

Particle Lot Description: Composite of Buffer Coated BWXT Kernel Composite 69300

Filename: IImc-agr|AGR\ParticleWeight|W05062201_DRF22R1.xIs

\begin{tabular}{|r|c|c|c|c|c|}
\hline & Sample 1 & Sample 2 & Sample 3 & Sample 4 & Sample 5 \\
\hline Weight of particles (g): & $3.35 \mathrm{E}-02$ & $3.07 \mathrm{E}-02$ & $4.09 \mathrm{E}-02$ & $3.50 \mathrm{E}-02$ & $4.08 \mathrm{E}-02$ \\
\hline Number of particles: & 109 & 99 & 132 & 113 & 132 \\
\hline Average weight/particle (g): & $3.07 \mathrm{E}-04$ & $3.10 \mathrm{E}-04$ & $3.10 \mathrm{E}-04$ & $3.10 \mathrm{E}-04$ & $3.09 \mathrm{E}-04$ \\
\hline
\end{tabular}

Mean average weight/particle $(\mathrm{g}): 3.09 \mathrm{E}-04$

Uncertainty in mean average weight/particle (g): $4.99 \mathrm{E}-07$

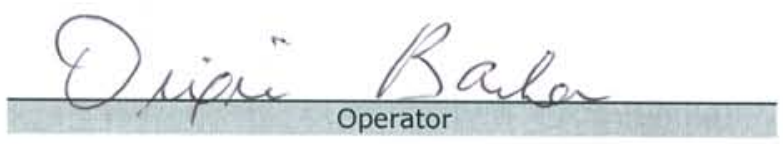

$$
6-22-05
$$

Date 
Data Report Form DRF-10A: Measurement of Particle Diameter

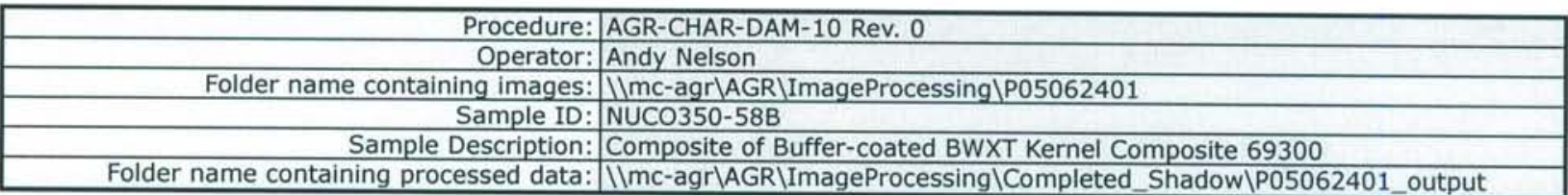

Number of particles analyzed: 640

Mean of the average diameter of each particle $(\mu \mathrm{m}): 561$

Standard deviation in the average diameter of each particle $(\mu \mathrm{m}): 18$

Distribution of the average particle diameter (top binned)

\begin{tabular}{|rr|}
\hline Mean Diameter & Frequency \\
\hline$<500$ & 0 \\
510 & 2 \\
520 & 7 \\
530 & 24 \\
540 & 49 \\
550 & 86 \\
560 & 127 \\
570 & 158 \\
580 & 102 \\
590 & 55 \\
600 & 21 \\
610 & 5 \\
620 & 0 \\
630 & 2 \\
640 & 0 \\
650 & 2 \\
$>650$ & 0 \\
\hline
\end{tabular}

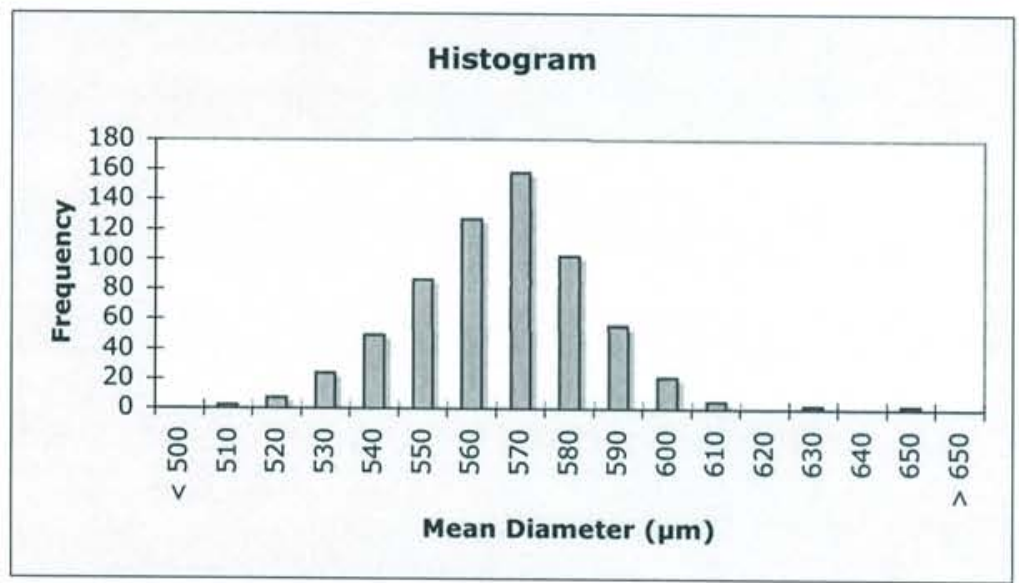

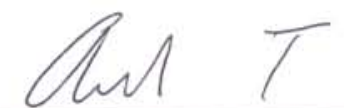

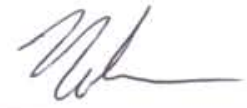

Operator

$$
6 / 27 / 2005
$$


ORNL/TM-2006/019, Rev. 1

Data Report Form DRF-16: Measurement of Buffer Envelope Density using a Mercury Porosimeter

\begin{tabular}{|c|c|}
\hline Procedure: & AGR-CHAR-DAM-16 Rev, 2 \\
\hline Operator: & S. D. NUNN \\
\hline Buffer-coated kernel batch ID: & LEU01-16B \\
\hline Batch Description: & Buffer on BWXT LEUCO kernel composite 69302 \\
\hline Thermocouple Expiration Date: & $5 / 23 / 06$ \\
\hline Penetrometer Expiration Date: & $5 / 25 / 06$ \\
\hline Completed DRF Filename: & 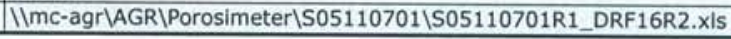 \\
\hline
\end{tabular}

\begin{tabular}{|r|l|}
\hline Mean average weight/buffer-coated kernel $(\mathrm{g}):$ & $3.20 \mathrm{E}-04$ \\
\hline Uncertainty in mean average weight/b-c kernel $(\mathrm{g}):$ & $8.22 \mathrm{E}-07$ \\
\hline Mean average weight/bare kernel $(\mathrm{g}):$ & $2.42 \mathrm{E}-04$ \\
\hline Uncertainty in mean average weight/bare kernel $(\mathrm{g}):$ & $5.96 \mathrm{E}-07$ \\
\hline Mean average envelope volume/bare kernel $(\mathrm{cc}):$ & $2.21 \mathrm{E}-05$ \\
\hline Uncertainty in envelope volume/bare kernel $(\mathrm{cc}):$ & $1.34 \mathrm{E}-08$ \\
\hline
\end{tabular}

\begin{tabular}{|r|c|c|c|c|c|}
\cline { 2 - 5 } \multicolumn{1}{c|}{} & Sample 1 & Sample 2 & Sample 3 & Sample 4 & Sample 5 \\
\hline Porosimeter data file number: & S05110701L & S05110702L & S05110703L & S05110704L & S05110705L \\
\hline Weight of buffer-coated kernels $(\mathrm{g}):$ & 4.0951 & 4.1007 & 4.2203 & 4.2527 & 4.3448 \\
\hline Approximate number of b-c kernels: & 12797 & 12815 & 13188 & 13290 & 13578 \\
\hline Uncertainty in number of b-c kernels: & 33 & 33 & 34 & 34 & 35 \\
\hline Total envelope volume of sample (cc): & 1.192 & 1.193 & 1.234 & 1.229 & 1.267 \\
\hline Av. envelope volume/b-c kernels (cc): & $9.32 \mathrm{E}-05$ & $9.31 \mathrm{E}-05$ & $9.36 \mathrm{E}-05$ & $9.25 \mathrm{E}-05$ & $9.33 \mathrm{E}-05$ \\
\hline Sample envelope density $(\mathrm{g} / \mathrm{cc}):$ & 3.435 & 3.438 & 3.421 & 3.460 & 3.430 \\
\hline
\end{tabular}

Mean average envelope volume/b-c kernel (cc): $9.31 \mathrm{E}-05$ Uncertainty in envelope volume/b-c kernel (cc): $1.8 \mathrm{E}-07$

Buffer density: $1.10 \mathrm{E}+00$

Uncertainty in buffer density: $1.46 \mathrm{E}-02$

S.D.Dlun Operator
$2 / 14106$

Date 
ORNL/TM-2006/019, Rev. 1

Data Report Form DRF-22: Estimation of Average Particle Weight

Procedure: AGR-CHAR-DAM-22 Rev. 1

Particle Lot ID: LEU 01-16B

Particle Lot Description: Buffer on BWXT kernel composite 69302

Filename: |IMc-agr|AGRIParticleWeight\W05110701_DRF22R1.xIs

\begin{tabular}{l}
\begin{tabular}{|r|c|c|c|c|c|}
\hline & Sample 1 & Sample 2 & Sample 3 & Sample 4 & Sample 5 \\
\hline Weight of particles (g): & $6.21 \mathrm{E}-02$ & $6.72 \mathrm{E}-02$ & $5.58 \mathrm{E}-02$ & $5.12 \mathrm{E}-02$ & $6.32 \mathrm{E}-02$ \\
\hline Number of particles: & 194 & 209 & 175 & 159 & 199 \\
\hline Average weight/particle (g): & $3.20 \mathrm{E}-04$ & $3.22 \mathrm{E}-04$ & $3.19 \mathrm{E}-04$ & $3.22 \mathrm{E}-04$ & $3.18 \mathrm{E}-04$ \\
\hline
\end{tabular} \\
\begin{aligned} & \hline Mean average weight/particle (g): $3.20 \mathrm{E}-04 \\
&$\hline\end{aligned} \\
Uncertainty in mean average weight/particle (g): $8.22 \mathrm{E}-07$ \\
\hline
\end{tabular}

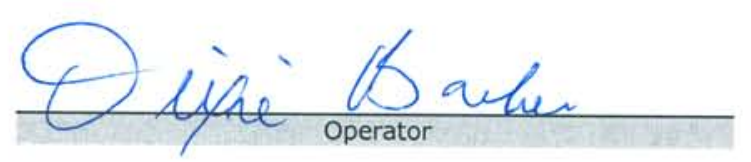

$$
11-n-0.5
$$

66 


\section{Characterization of IPyC on interrupted batches}

This section contains data on the inner pyrocarbon (IPyC) coating density and other properties for particles removed from the coater after deposition of only the buffer and IPyC layers. The data was obtained according to product inspection plan AGR-CHAR-PIP-02R4. The IPyC on these particles was deposited under similar conditions as the IPyC on the particles in composite LEU01-46T and should therefore be representative of the IPyC on the particles in that composite. Density measurements were made on these interrupted batches because it was not feasible to measure the IPyC density after all coating layers were applied. According to section 5.3 of EDF-4380, Rev. 6, three representative IPyC layers from interrupted batches which meet the specification for IPyC density are sufficient to qualify the process for IPyC density. The qualifying batches used $350 \mu \mathrm{m}$ natural uranium oxide/uranium carbide kernels (NUCO). The kernels were obtained from BWXT and were identified as composite G73B-NU-69300. The use of NUCO surrogate kernels was not expected to significantly effect the density of the IPyC. A confirmatory batch using LEUCO kernels from G73D-20-69302 was coated and characterized to verify this assumption.

The following page shows the inspection report form (IRF-02B). Following IRF-02B are the individual data report forms for the measurements that were performed. Additional data at the end of this section is provided for information only. The process conditions used to deposit the IPyC layer were determined to be sufficient to satisfy the specifications in section 5.3 of EDF 4380, Rev. 6. 
ORNL/TM-2006/019, Rev. 1

Inspection Report Form IRF-02B: Interrupted Coating Batches - IPYC Density

\begin{tabular}{|c|c|}
\hline Procedure: & AGR-CHAR-PIP-02 Rev. 4 \\
\hline Batch 1 ID: & NUCO $350-30 \mathrm{BI}$ \\
\hline Batch 1 description: & IPyC/Buffer on BWXT kernel composite 69300 \\
\hline Batch 2 ID: & NUCO350-37BI \\
\hline Batch 2 description: & IPyC/Buffer on BWXT kernel composite 69300 \\
\hline Batch 3 ID: & NUCO $350-298 I$ \\
\hline Batch 3 description: & IPyC/Buffer on BWXT kernel composite 69300 \\
\hline
\end{tabular}

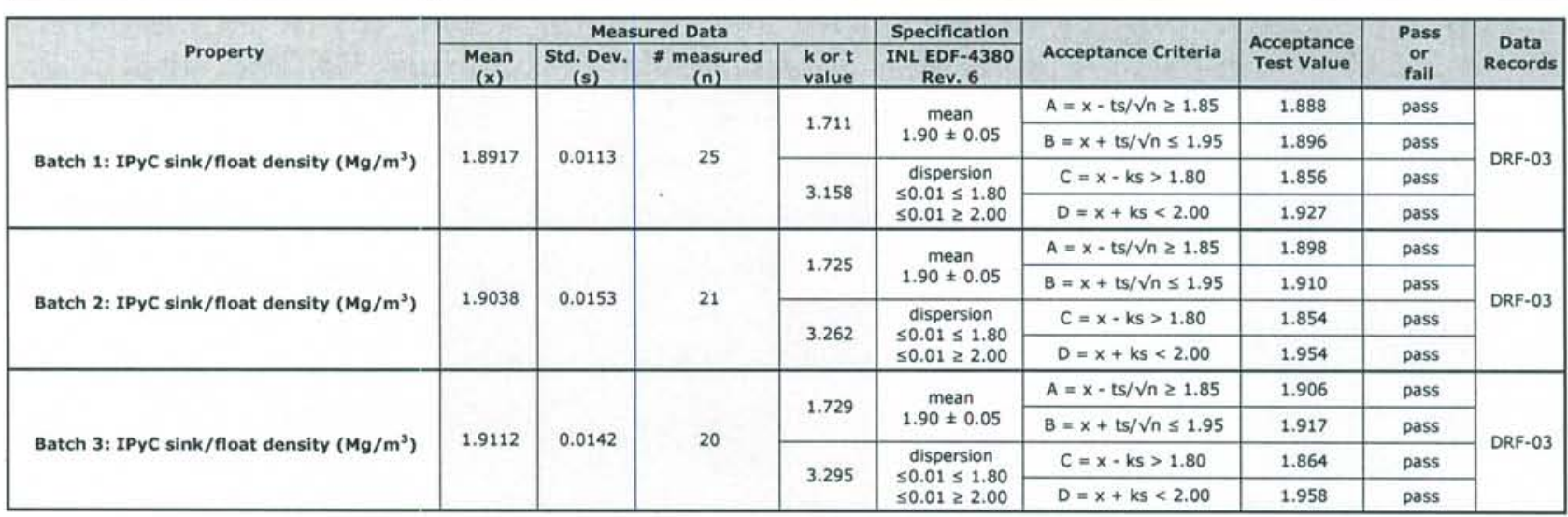

interval for Buffer thickness in composite $=(104 \mu \mathrm{m}, 106 \mu \mathrm{m})$ with $<1 \% \leq 55 \mu \mathrm{m}$.

$95 \%$ confidence interval for IPyC thickness in composite $=(34.2 \mu \mathrm{m}, 34.9 \mu \mathrm{m})$ with $>1 \% \leq 30 \mu \mathrm{m}$ and $<1 \% \geq 56 \mu \mathrm{m}$.

Confirmatory batch on LEUCO kernels, LEU01-15t: mean IPyC density $=1.9074 \mathrm{~g} / \mathrm{cc}$.

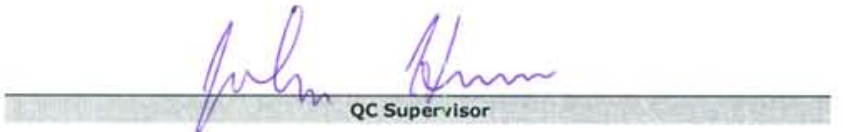

Accept process for IPyC density (Yes or No)

Yes

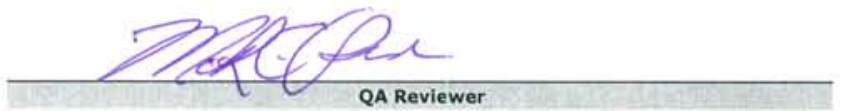

$3-10-06$

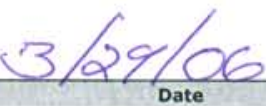


Data Report Form DRF-03: Measurement of PyC Density using a Density Gradient Column

\begin{tabular}{|r|l|}
\hline Procedure: & AGR-CHAR-DAM-03 Rev. 1 \\
\hline Operator: & Dixie Barker \\
\hline Filename: & IImC-agr\AGR\DensityColumn\D05062901_DRF03R1.xIs \\
\hline Sample ID: & NUCO350-30BI-B \\
\hline Sample Description: & IPyC/Buffer on BWXT kernel composite 69300 \\
\hline Float Expiration Date: & $07 / 2007$ \\
\hline Bath Temperature: & 23.1C \\
\hline
\end{tabular}

\begin{tabular}{|c|c|r|r|}
\hline \multicolumn{4}{|c|}{ Calibrated Floats } \\
\hline Density & $\begin{array}{c}\text { Top of } \\
\text { Float }\end{array}$ & $\begin{array}{c}\text { Bottom of } \\
\text { Float }\end{array}$ & $\begin{array}{c}\text { Center of } \\
\text { Mass }\end{array}$ \\
\hline \hline 1.800 & 154.64 & 163.83 & 159.24 \\
\hline 1.850 & 258.61 & 266.87 & 262.74 \\
\hline 1.900 & 361.46 & 367.13 & 364.30 \\
\hline 1.950 & 459.26 & 467.16 & 463.21 \\
\hline 2.000 & 553.39 & 560.32 & 556.86 \\
\hline
\end{tabular}

\begin{tabular}{|c|c|c|c|}
\hline \multicolumn{4}{|c|}{ Linear Fit } \\
\hline slope & StDev & intercept & StDev \\
\hline \hline $5.02 \mathrm{E}-04$ & $3.18 \mathrm{E}-06$ & $1.72 \mathrm{E}+00$ & $1.25 \mathrm{E}-03$ \\
\hline
\end{tabular}

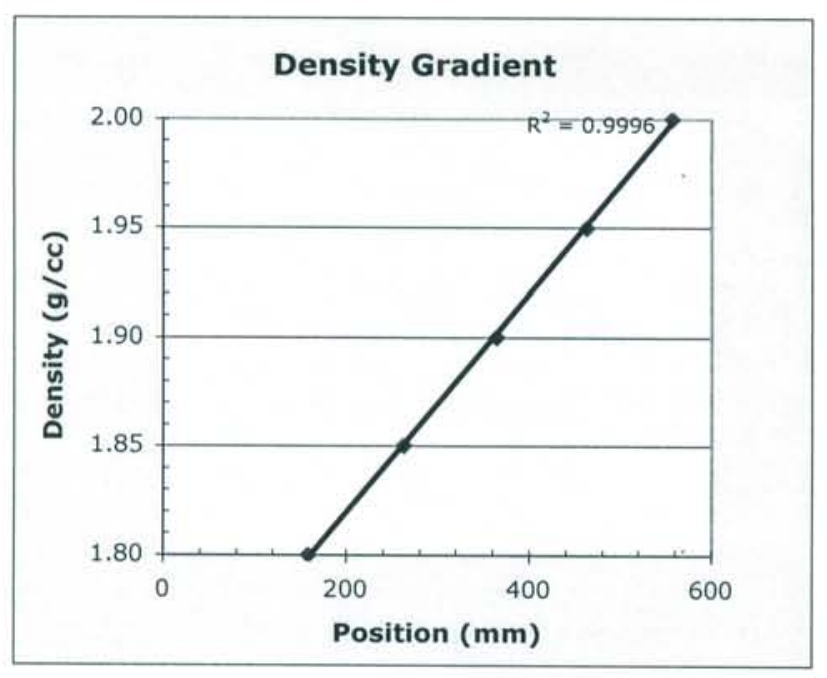

\begin{tabular}{|c|c|c|c|}
\hline \multicolumn{4}{|c|}{ Sample Density } \\
\hline $\begin{array}{l}\text { Particle } \\
\text { Number }\end{array}$ & $\begin{array}{l}\text { Particle } \\
\text { Position }\end{array}$ & $\begin{array}{c}\text { Calculated } \\
\text { Density }\end{array}$ & $\begin{array}{c}\text { Standard } \\
\text { Error }\end{array}$ \\
\hline 1 & 295.22 & 1.8668 & 0.0016 \\
\hline 2 & 304.23 & 1.8714 & 0.0016 \\
\hline 3 & 325.99 & 1.8823 & 0.0016 \\
\hline 4 & 326.44 & 1.8825 & 0.0016 \\
\hline 5 & 326.76 & 1.8827 & 0.0016 \\
\hline 6 & 328.81 & 1.8837 & 0.0016 \\
\hline 7 & 326.67 & 1.8826 & 0.0016 \\
\hline 8 & 333.83 & 1.8862 & 0.0016 \\
\hline 9 & 335.75 & 1.8872 & 0.0016 \\
\hline 10 & 334.07 & 1.8863 & 0.0016 \\
\hline 11 & 335.11 & 1.8869 & 0.0016 \\
\hline 12 & 336.64 & 1.8876 & 0.0016 \\
\hline 13 & 348.87 & 1.8938 & 0.0017 \\
\hline 14 & 348.31 & 1.8935 & 0.0017 \\
\hline 15 & 350.33 & 1.8945 & 0.0017 \\
\hline 16 & 351.97 & 1.8953 & 0.0017 \\
\hline 17 & 355.50 & 1.8971 & 0.0017 \\
\hline 18 & 357.75 & 1.8982 & 0.0017 \\
\hline 19 & 359.46 & 1.8991 & 0.0017 \\
\hline 20 & 364.06 & 1.9014 & 0.0017 \\
\hline 21 & 363.84 & 1.9013 & 0.0017 \\
\hline 22 & 362.42 & 1.9006 & 0.0017 \\
\hline 23 & 368.25 & 1.9035 & 0.0017 \\
\hline 24 & 385.58 & 1.9122 & 0.0018 \\
\hline 25 & 390.04 & 1.9144 & 0.0018 \\
\hline Average Den & & 1.8917 & 0.0003 \\
\hline Standard De & & 0.0113 & \\
\hline
\end{tabular}

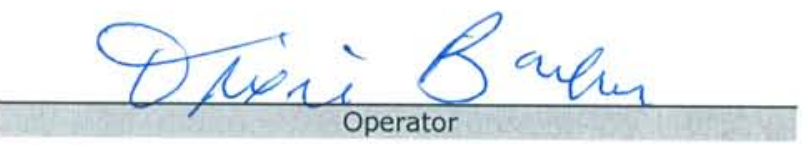

$\frac{6-29-05}{\text { Date }}$ 
Data Report Form DRF-03: Measurement of PyC Density using a Density Gradient Column

\begin{tabular}{|r|l|}
\hline Procedure: & AGR-CHAR-DAM-03 Rev. 1 \\
\hline Operator: & Dixie Barker \\
\hline Filename: & IImC-agr\AGRIDensityColumn\D05063001_DRF03R1.xIs \\
\hline Sample ID: & NUCO350-37BI-B \\
\hline Sample Description: & IPyC/Buffer on BWXT kernel composite 69300 \\
\hline Float Expiration Date: & $07 / 2007$ \\
\hline Gauge Expiration Date: & $01 / 2006$ \\
\hline Bath Temperature: & $22.9 \mathrm{C}$ \\
\hline
\end{tabular}

\begin{tabular}{|c|c|c|c|}
\hline \multicolumn{4}{|c|}{ Calibrated Floats } \\
\hline Density & $\begin{array}{c}\text { Top of } \\
\text { Float }\end{array}$ & $\begin{array}{c}\text { Bottom of } \\
\text { Float }\end{array}$ & $\begin{array}{c}\text { Center of } \\
\text { Mass }\end{array}$ \\
\hline \hline 1.800 & 151.98 & 161.42 & 156.70 \\
\hline 1.850 & 262.34 & 270.69 & 266.52 \\
\hline 1.900 & 370.32 & 376.20 & 373.26 \\
\hline 1.950 & 473.40 & 480.73 & 477.07 \\
\hline 2.000 & 569.44 & 576.52 & 572.98 \\
\hline
\end{tabular}

\begin{tabular}{|c|c|c|c|}
\hline \multicolumn{4}{|c|}{ Linear Fit } \\
\hline slope & StDev & intercept & StDev \\
\hline \hline $4.79 \mathrm{E}-04$ & $2.94 \mathrm{E}-06$ & $1.72 \mathrm{E}+00$ & $1.17 \mathrm{E}-03$ \\
\hline
\end{tabular}

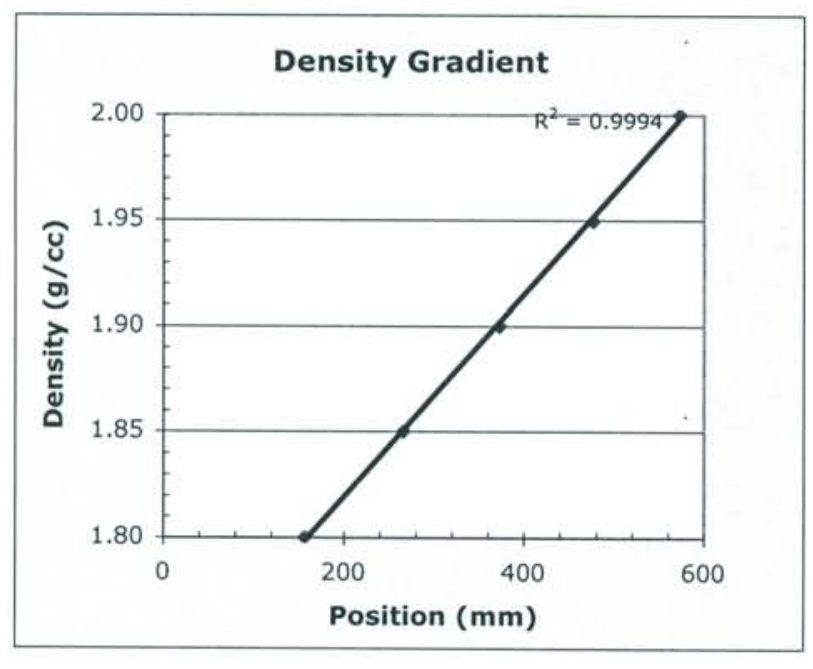

\begin{tabular}{|c|c|c|c|}
\hline \multicolumn{4}{|c|}{ Sample Density } \\
\hline $\begin{array}{l}\text { Particle } \\
\text { Number }\end{array}$ & $\begin{array}{l}\text { Particle } \\
\text { Position }\end{array}$ & $\begin{array}{c}\text { Calculated } \\
\text { Density }\end{array}$ & $\begin{array}{l}\text { Standard } \\
\text { Error }\end{array}$ \\
\hline 1 & 313.15 & 1.8731 & 0.0015 \\
\hline 2 & 329.08 & 1.8807 & 0.0015 \\
\hline 3 & 335.13 & 1.8836 & 0.0015 \\
\hline 4 & 342.49 & 1.8872 & 0.0015 \\
\hline 5 & 349.04 & 1.8903 & 0.0016 \\
\hline 6 & 351.34 & 1.8914 & 0.0016 \\
\hline 7 & 365.21 & 1.8980 & 0.0016 \\
\hline 8 & 367.09 & 1.8989 & 0.0016 \\
\hline 9 & 367.27 & 1.8990 & 0.0016 \\
\hline 10 & 378.19 & 1.9043 & 0.0016 \\
\hline 11 & 382.30 & 1.9062 & 0.0016 \\
\hline 12 & 386.45 & 1.9082 & 0.0016 \\
\hline 13 & 387.67 & 1.9088 & 0.0016 \\
\hline 14 & 389.34 & 1.9096 & 0.0016 \\
\hline 15 & 390.40 & 1.9101 & 0.0016 \\
\hline 16 & 404.73 & 1.9170 & 0.0017 \\
\hline 17 & 409.36 & 1.9192 & 0.0017 \\
\hline 18 & 412.62 & 1.9207 & 0.0017 \\
\hline 19 & 412.95 & 1.9209 & 0.0017 \\
\hline 20 & 418.50 & 1.9236 & 0.0017 \\
\hline 21 & 428.34 & 1.9283 & 0.0017 \\
\hline \multicolumn{4}{|l|}{22} \\
\hline \multicolumn{4}{|l|}{23} \\
\hline \multicolumn{4}{|l|}{24} \\
\hline \multicolumn{4}{|l|}{25} \\
\hline \multicolumn{2}{|c|}{ Average Density } & 1.9038 & 0.0004 \\
\hline \multicolumn{2}{|c|}{ Standard Deviation } & 0.0153 & 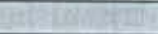 \\
\hline
\end{tabular}
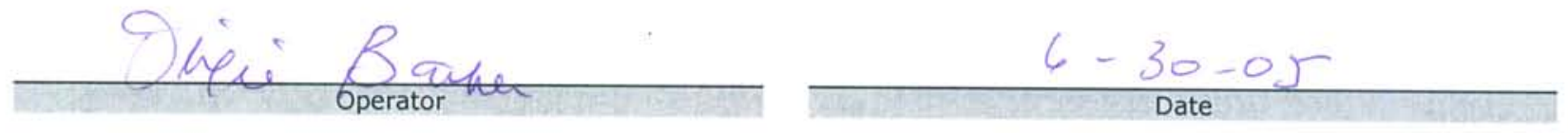
Data Report Form DRF-03: Measurement of PyC Density using a Density Gradient Column

\begin{tabular}{|r|l|}
\hline Procedure: & AGR-CHAR-DAM-03 Rev. 1 \\
\hline Operator: & Dixie Barker \\
\hline Filename: & IImC-agr\AGRIDensityColumn\D05070101_DRF03R1.xls \\
\hline Sample ID: & NUCO350-29BI-B \\
\hline Sample Description: & Ipyc/Buffer on BWXT kernel composite 69300 \\
\hline Float Expiration Date: & $07 / 2007$ \\
\hline Bauge Expiration Date: & $01 / 2006$ \\
\hline
\end{tabular}

\begin{tabular}{|c|c|c|c|}
\hline \multicolumn{4}{|c|}{ Calibrated Floats } \\
\hline Density & $\begin{array}{c}\text { Top of } \\
\text { Float }\end{array}$ & $\begin{array}{c}\text { Bottom of } \\
\text { Float }\end{array}$ & $\begin{array}{c}\text { Center of } \\
\text { Mass }\end{array}$ \\
\hline \hline 1.800 & 145.75 & 154.69 & 150.22 \\
\hline 1.850 & 254.86 & 262.94 & 258.90 \\
\hline 1.900 & 363.03 & 368.69 & 365.86 \\
\hline 1.950 & 466.04 & 473.41 & 469.73 \\
\hline 2.000 & 561.98 & 569.07 & 565.53 \\
\hline
\end{tabular}

\begin{tabular}{|c|c|c|c|}
\hline \multicolumn{4}{|c|}{ Linear Fit } \\
\hline slope & StDev & intercept & StDev \\
\hline \hline $4.80 \mathrm{E}-04$ & $3.17 \mathrm{E}-06$ & $1.73 \mathrm{E}+00$ & $1.15 \mathrm{E}-03$ \\
\hline
\end{tabular}

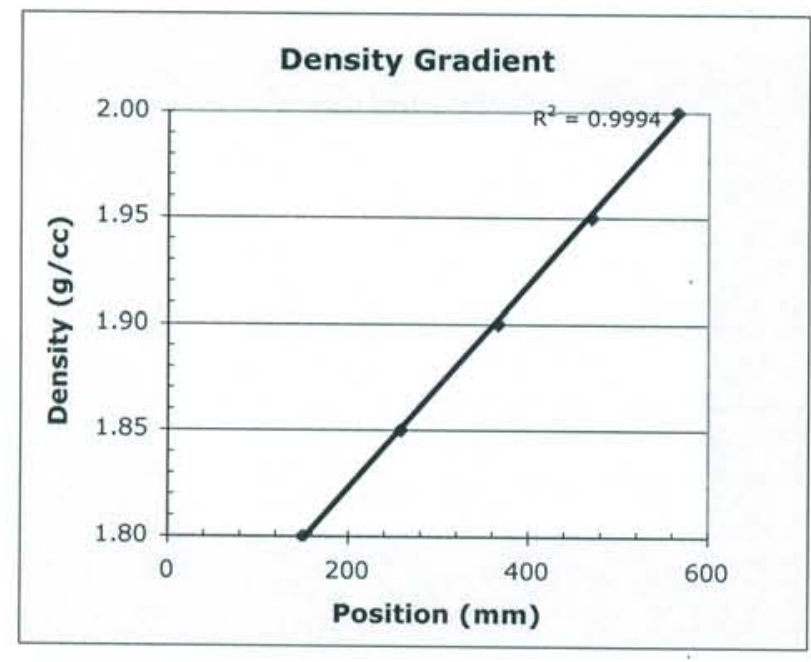

\begin{tabular}{|c|c|c|c|}
\hline \multicolumn{4}{|c|}{ Sample Density } \\
\hline $\begin{array}{c}\text { Particle } \\
\text { Number }\end{array}$ & $\begin{array}{l}\text { Particle } \\
\text { Position } \\
\end{array}$ & $\begin{array}{c}\text { Calculated } \\
\text { Density } \\
\end{array}$ & $\begin{array}{c}\text { Standard } \\
\text { Error }\end{array}$ \\
\hline 1 & 321.45 & 1.8805 & 0.0015 \\
\hline 2 & 336.71 & 1.8878 & 0.0016 \\
\hline 3 & 341.90 & 1.8903 & 0.0016 \\
\hline 4 & 358.02 & 1.8981 & 0.0016 \\
\hline 5 & 368.37 & 1.9030 & 0.0016 \\
\hline 6 & 374.74 & 1.9061 & 0.0017 \\
\hline 7 & 373.30 & 1.9054 & 0.0017 \\
\hline 8 & 379.31 & 1.9083 & 0.0017 \\
\hline 9 & 382.20 & 1.9097 & 0.0017 \\
\hline 10 & 383.00 & 1.9101 & 0.0017 \\
\hline 11 & 390.54 & 1.9137 & 0.0017 \\
\hline 12 & 390.54 & 1.9137 & 0.0017 \\
\hline 13 & 405.88 & 1.9210 & 0.0017 \\
\hline 14 & 406.48 & 1.9213 & 0.0017 \\
\hline 15 & 408.91 & 1.9225 & 0.0017 \\
\hline 16 & 409.63 & 1.9228 & 0.0017 \\
\hline 17 & 414.97 & 1.9254 & 0.0017 \\
\hline 18 & 423.66 & 1.9296 & 0.0018 \\
\hline 19 & 429.22 & 1.9322 & 0.0018 \\
\hline 20 & 410.67 & 1.9233 & 0.0017 \\
\hline \multicolumn{4}{|l|}{21} \\
\hline \multirow{2}{*}{\multicolumn{4}{|c|}{$\frac{22}{23}$}} \\
\hline 23 & & & \\
\hline \multicolumn{4}{|l|}{24} \\
\hline \multicolumn{4}{|l|}{25} \\
\hline Average Den & & 1.9112 & 0.0004 \\
\hline Standard De & & 0.0142 & + \\
\hline
\end{tabular}

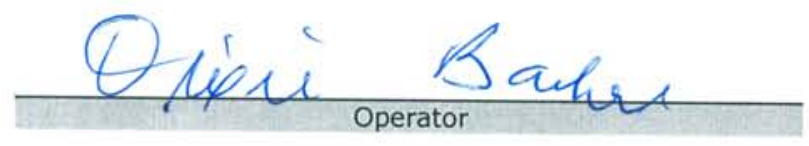

$\frac{\eta-1-05}{\text { Date }}$ 


\section{For Information Only}

The information in the remainder of this section reports results of measurements not required by the fuel specification and is provided for information only. 


\begin{tabular}{|r|l|}
\hline Procedure: & AGR-CHAR-DAM-03 Rev. 2 \\
\hline Operator: & Dixie Barker \\
\hline Filename: & IImC-agr\AGRIDensityColumn\D05110801_DRF03R2.xIs \\
\hline Sample ID: & LEU01-15I-B01 \\
\hline Sample description: & IPyC/Buffer on BWXT kernel composite 69302 \\
\hline Float expiration date: & $07 / 2007$ \\
\hline Gauge expiration date: & $01 / 2006$ \\
\hline Bath temperature: & $22.7^{\circ} \mathrm{C}$ \\
\hline
\end{tabular}

\begin{tabular}{|c|c|c|c|}
\hline \multicolumn{4}{|c|}{ Calibrated Floats } \\
\hline Density & $\begin{array}{c}\text { Top of } \\
\text { Float }\end{array}$ & $\begin{array}{c}\text { Bottom of } \\
\text { Float }\end{array}$ & $\begin{array}{c}\text { Center of } \\
\text { Mass }\end{array}$ \\
\hline \hline 1.800 & 134.80 & 144.21 & 139.51 \\
\hline 1.850 & 243.34 & 251.54 & 247.44 \\
\hline 1.900 & 351.28 & 357.11 & 354.20 \\
\hline 1.950 & 456.05 & 463.23 & 459.64 \\
\hline 2.000 & 557.73 & 565.05 & 561.39 \\
\hline
\end{tabular}

\begin{tabular}{|c|c|c|c|}
\hline \multicolumn{4}{|c|}{ Linear Fit } \\
\hline slope & StDev & intercept & StDev \\
\hline \hline $4.73 \mathrm{E}-04$ & $2.74 \mathrm{E}-06$ & $1.73 \mathrm{E}+00$ & $9.73 \mathrm{E}-04$ \\
\hline
\end{tabular}

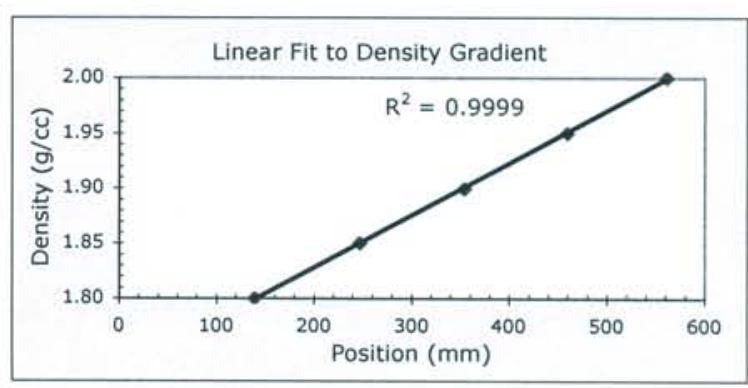

\begin{tabular}{|c|c|c|c|c|c|c|c|c|}
\hline $\begin{array}{c}\text { Fragment } \\
\text { Number }\end{array}$ & $\begin{array}{c}\text { Fragment } \\
\text { Position }\end{array}$ & $\begin{array}{c}\text { Calculated } \\
\text { Density } \\
\end{array}$ & $\begin{array}{c}\text { Fragment } \\
\text { Number }\end{array}$ & $\begin{array}{c}\text { Fragment } \\
\text { Position }\end{array}$ & $\begin{array}{c}\text { Calculated } \\
\text { Density }\end{array}$ & $\begin{array}{c}\text { Fragment } \\
\text { Number }\end{array}$ & $\begin{array}{c}\text { Fragment } \\
\text { Position }\end{array}$ & $\begin{array}{c}\text { Calculated } \\
\text { Density } \\
\end{array}$ \\
\hline 1 & 304.62 & 1.8774 & 26 & 370.16 & 1.9084 & 51 & & \\
\hline 2 & 311.60 & 1.8807 & 27 & 370.85 & 1.9087 & 52 & & \\
\hline 3 & 316.86 & 1.8832 & 28 & 369.53 & 1.9081 & 53 & & \\
\hline 4 & 324.68 & 1.8869 & 29 & 374.00 & 1.9102 & 54 & & \\
\hline 5 & 328.13 & 1.8885 & 30 & 375.99 & 1.9112 & 55 & & \\
\hline 6 & 332.97 & 1.8908 & 31 & 381.77 & 1.9139 & 56 & & \\
\hline 7 & 334.87 & 1.8917 & 32 & 382.71 & 1.9143 & 57 & & \\
\hline 8 & 338.02 & 1.8932 & 33 & 384.42 & 1.9151 & 58 & & \\
\hline 9 & 338.84 & 1.8936 & 34 & 385.50 & 1.9157 & 59 & & \\
\hline 10 & 340.51 & 1.8944 & 35 & 386.44 & 1.9161 & 60 & & \\
\hline 11 & 341.77 & 1.8950 & 36 & 386.80 & 1.9163 & 61 & & \\
\hline 12 & 341.09 & 1.8946 & 37 & 387.69 & 1.9167 & 62 & & \\
\hline 13 & 341.09 & 1.8946 & 38 & 388.54 & 1.9171 & 63 & & \\
\hline 14 & 351.54 & 1.8996 & 39 & 391.14 & 1.9183 & 64 & & \\
\hline 15 & 352.97 & 1.9003 & 40 & 392.11 & 1.9188 & 65 & & \\
\hline 16 & 354.39 & 1.9009 & 41 & 393.13 & 1.9193 & 66 & & \\
\hline 17 & 358.58 & 1.9029 & 42 & 396.08 & 1.9207 & 67 & & \\
\hline 18 & 359.74 & 1.9035 & 43 & 397.78 & 1.9215 & 68 & & \\
\hline 19 & 358.62 & 1.9029 & 44 & 399.01 & 1.9221 & 69 & & \\
\hline 20 & 360.57 & 1.9039 & 45 & 400.37 & 1.9227 & 70 & & \\
\hline 21 & 361.40 & 1.9042 & 46 & 403.99 & 1.9244 & 71 & & \\
\hline 22 & 362.69 & 1.9049 & 47 & 407.97 & 1.9263 & 72 & & \\
\hline 23 & 365.05 & 1.9060 & 48 & 408.28 & 1.9264 & 73 & & \\
\hline 24 & 367.05 & 1.9069 & 49 & 420.44 & 1.9322 & 74 & & \\
\hline 25 & 369.10 & 1.9079 & 50 & 428.33 & 1.9359 & 75 & & \\
\hline \multicolumn{4}{|c|}{ Average density of PyC fragments: } & \multicolumn{5}{|c|}{1.9074} \\
\hline \multicolumn{4}{|c|}{ Standard deviation in density of PyC fragments: } & \multicolumn{5}{|c|}{0.0136} \\
\hline \multicolumn{4}{|c|}{ Uncertainty in calculated density of PyC fragments: } & \multicolumn{5}{|c|}{0.0015} \\
\hline
\end{tabular}
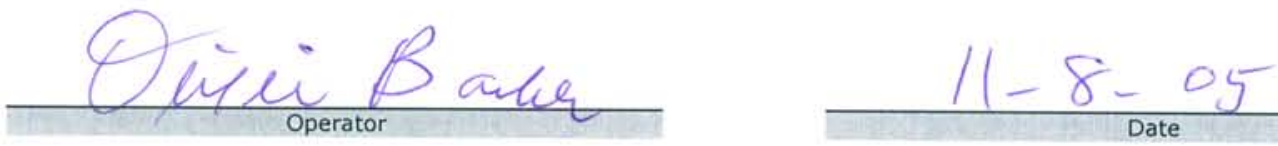
Summary of "info only" measurements

Coating Thickness

\begin{tabular}{|lccc|}
\hline & & Buffer thickness $(\mu \mathrm{m})$ & IPyC thickness $(\mu \mathrm{m})$ \\
\cline { 3 - 4 } Batch 1 & NUCO350-30BI-C & 99 & 36.3 \\
Batch 2 & NUCO350-37BI-C & 102 & 35.1 \\
Batch 3 & NUCO350-29BI-C & 107 & 33.9 \\
\hline Average & & 103 & 35.1 \\
& & & 34.5 \\
\hline
\end{tabular}

Open Porosity

\begin{tabular}{|c|c|c|c|}
\hline & & Average particle weight (g) & Open porosity $\left(\mathrm{ml} / \mathrm{m}^{2}\right)$ \\
\hline Composite & NUCO350-66BI & $3.95 \mathrm{E}-04$ & 1.65 \\
\hline Confirmatory & LEU01-15I & $4.26 \mathrm{E}-04$ & 1.56 \\
\hline
\end{tabular}

Note: The open porosity measurement on the IPyC was calculated from the mercury intrusion between 250 psi and 10000 psi per table 5.3 in EDF-4380, Rev. 6. In the upper half of this pressure range, there is some compression of the buffer layer that results in a value for open porosity that is higher than the actual value. The open porosity of the IPyC in LEU01-15I, calculated from the mercury intrusion between

$250 \mathrm{psi}$ and $5000 \mathrm{psi}$, was $1.20 \mathrm{ml} / \mathrm{m}^{2}$ which corresponds well with the open porosity measured for OPyC deposited under similar conditions. The open porosity of the IPyC in NUCO350-66BI, calculated from the

mercury intrusion between $250 \mathrm{psi}$ and $5000 \mathrm{psi}$, was $1.19 \mathrm{ml} / \mathrm{m}^{2}$. Porosimetry measurements on OPyC, where compression between 5000 psi and 10000 psi is negligible, show little mercury intrusion above 5000 psi. 
Data Report Form DRF-08: Imaging of Coated Particle Cross-sections Using an Optical Microscope System

\begin{tabular}{|r|l|}
\hline Procedure: & AGR-CHAR-DAM-08 Rev. 0 \\
\hline Operator: & Andrew K. Kercher \\
\hline Sample ID: & NUCO350-30BI-C \\
\hline Sample Description: & IPyC/Buffer on BWXT kernel composite $69300 \cdots$ repolished \\
\hline Mount Number(s): & M05062902 \\
\hline Folder name containing images: & IImC-agr\AGR\ImageProcessing $\backslash$ P05070101 \\
\hline
\end{tabular}

DMR Calibration Expiration Date: $3 / 24 / 06$

Stage Micrometer Calibration Expiration Date: $2 / 17 / 07$

Measured Value for $500 \mu \mathrm{m}$ in Stage Micrometer Image: $500.4 \mu \mathrm{m}$

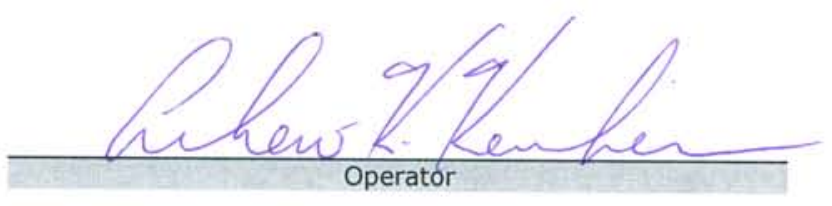

$$
\frac{07 / 01 / 05}{\text { Date }}
$$


Data Report Form DRF-11A: Measurement of Buffer Layer Thickness

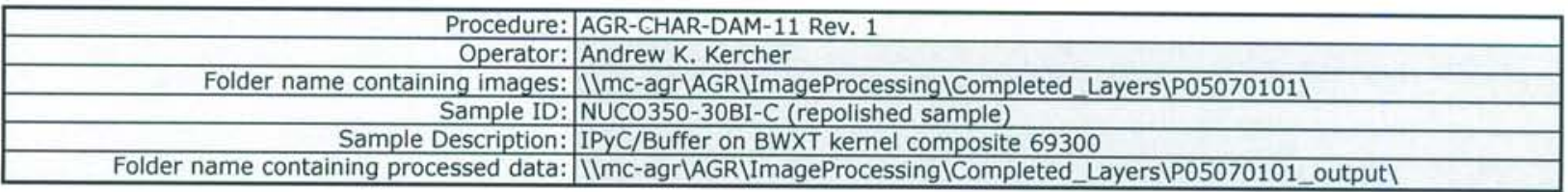

\begin{tabular}{r|r|}
\hline Number of buffer layers analyzed: & 123 \\
\hline Mean of the average buffer thickness of each particle $(\mu \mathrm{m}):$ & 99.4 \\
\hline Standard deviation in the average buffer thickness of each particle $(\mu \mathrm{m}):$ & 8.8 \\
\hline
\end{tabular}

Distribution of the average buffer layer thickness (top binned)

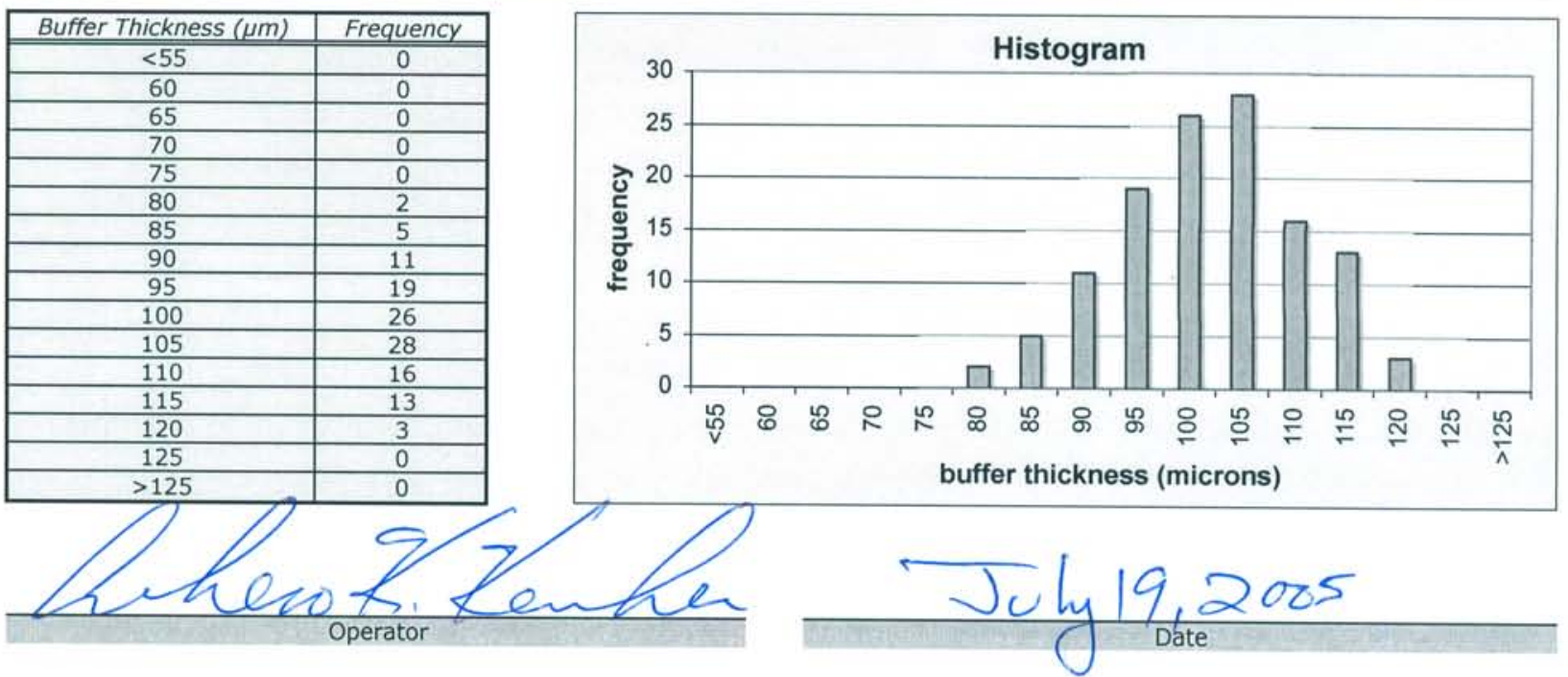


Data Report Form DRF-11B: Measurement of Inner Pyrocarbon Layer Thickness

\begin{tabular}{|c|c|}
\hline Procedure: & AGR-CHAR-DAM-11 Rev. 1 \\
\hline Operator: & Andrew K. Kercher \\
\hline Folder name containing images: & |Imc-agr|AGR|ImageProcessing|Completed_Layers|P05070101| \\
\hline Sample ID: & NUCO350-30BI-C (repolished sample) \\
\hline Sample Description: & IPyC/Buffer on BWXT kernel composite 69300 \\
\hline Folder name containing processed data: & IImc-agr|AGR\ImageProcessing|Completed_Layers\P05070101_output| \\
\hline
\end{tabular}

Number of inner pyrocarbon layers analyzed: $\quad 216$

Mean of the average IPyC thickness of each particle $(\mu \mathrm{m}): \quad 36.3$

Standard deviation in the average IPyC thickness of each particle $(\mu \mathrm{m})$ :

2.4

Distribution of the average IPyC layer thickness (top binned)

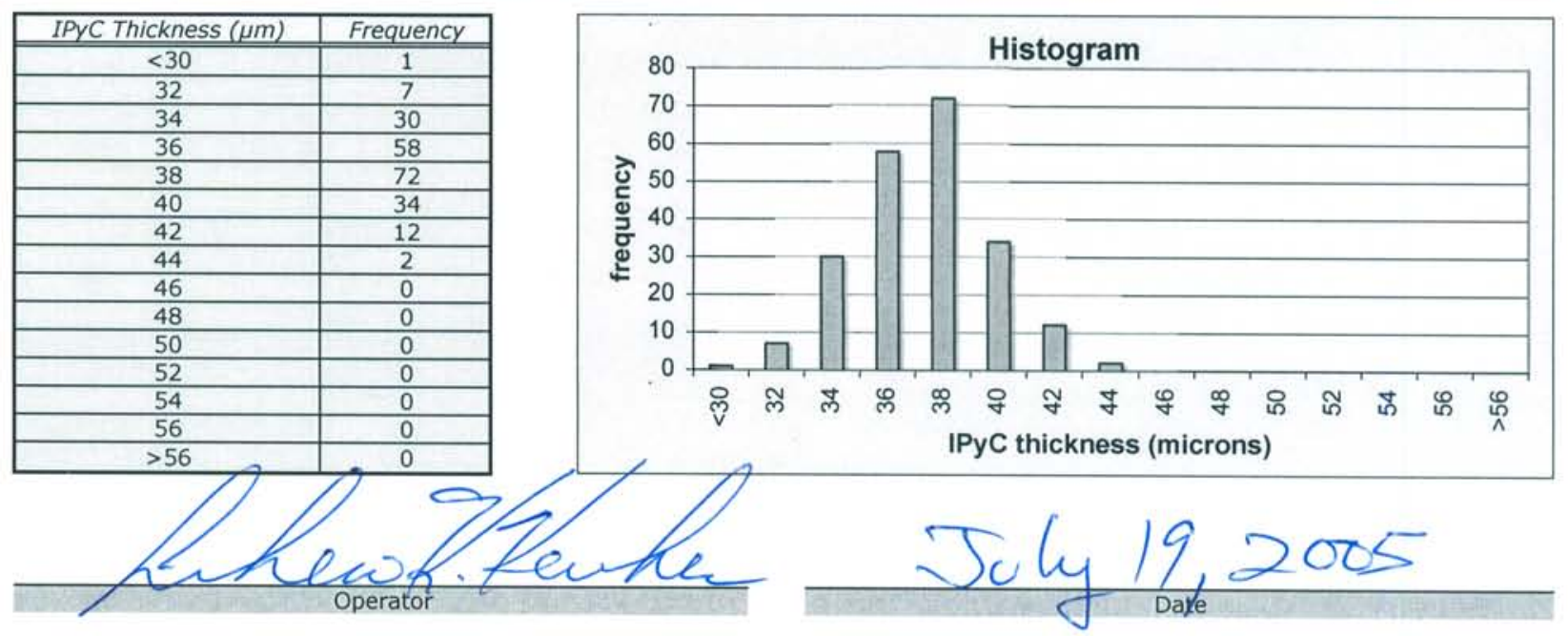


Data Report Form DRF-08: Imaging of Coated Particle Cross-sections Using an Optical Microscope System

\begin{tabular}{|r|l|}
\hline Procedure: & AGR-CHAR-DAM-08 Rev. 0 \\
\hline Operator: & Andrew K. Kercher \\
\hline Sample ID: & NUCO350-37BI-C \\
\hline Sample Description: & IPyC/Buffer on BWXT kernel composite 69300 \\
\hline Mount Number(s): & M05063001 \\
\hline Folder name containing images: & \Imc-agr\AGR\ImageProcessing\P05070501\} $\\
{\hline}$
\end{tabular}

DMR Calibration Expiration Date: $3 / 24 / 06$

Stage Micrometer Calibration Expiration Date: $2 / 17 / 07$

Measured Value for $500 \mu \mathrm{m}$ in Stage Micrometer Image: $500.7 \mu \mathrm{m}$
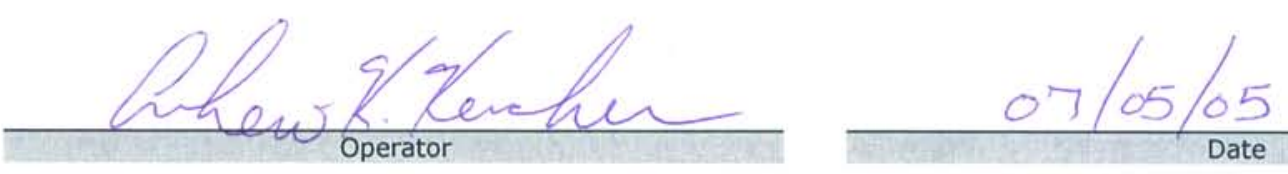


\section{Data Report Form DRF-11A: Measurement of Buffer Layer Thickness}

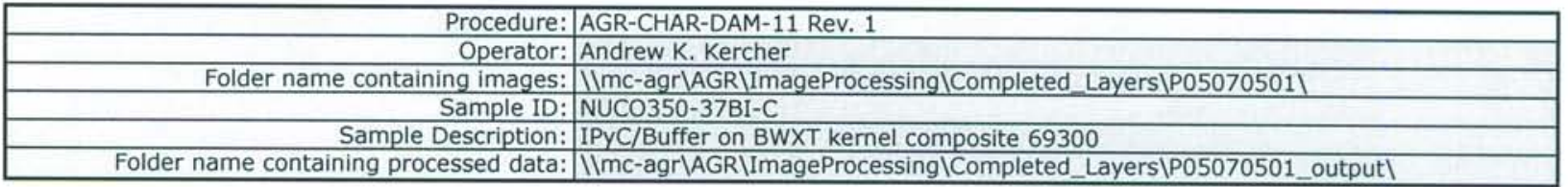

Number of buffer layers analyzed: $\quad 155$

Mean of the average buffer thickness of each particle $(\mu \mathrm{m}):$ : 101.5

Standard deviation in the average buffer thickness of each particle $(\mu \mathrm{m})$ :

8.9

\section{Distribution of the average buffer layer thickness (top binned)}

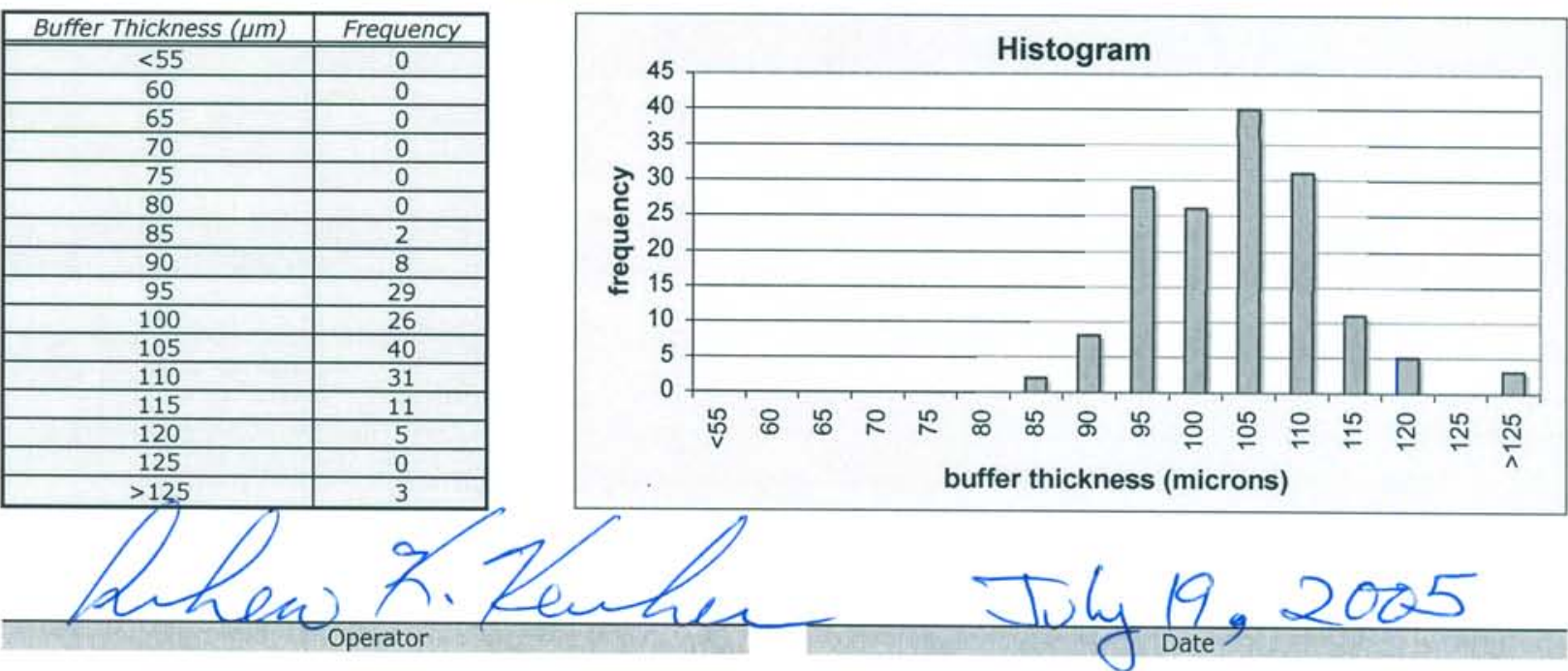


Data Report Form DRF-11B: Measurement of Inner Pyrocarbon Layer Thickness

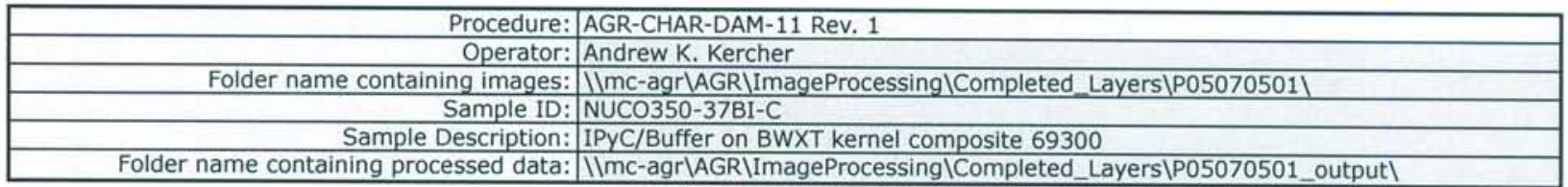

Number of inner pyrocarbon layers analyzed: $\quad 215$

Mean of the average IPyC thickness of each particle $(\mu \mathrm{m})$ :

Standard deviation in the average IPyC thickness of each particle $(\mu \mathrm{m})$ :

2.0

Distribution of the average IPYC layer thickness (top binned)

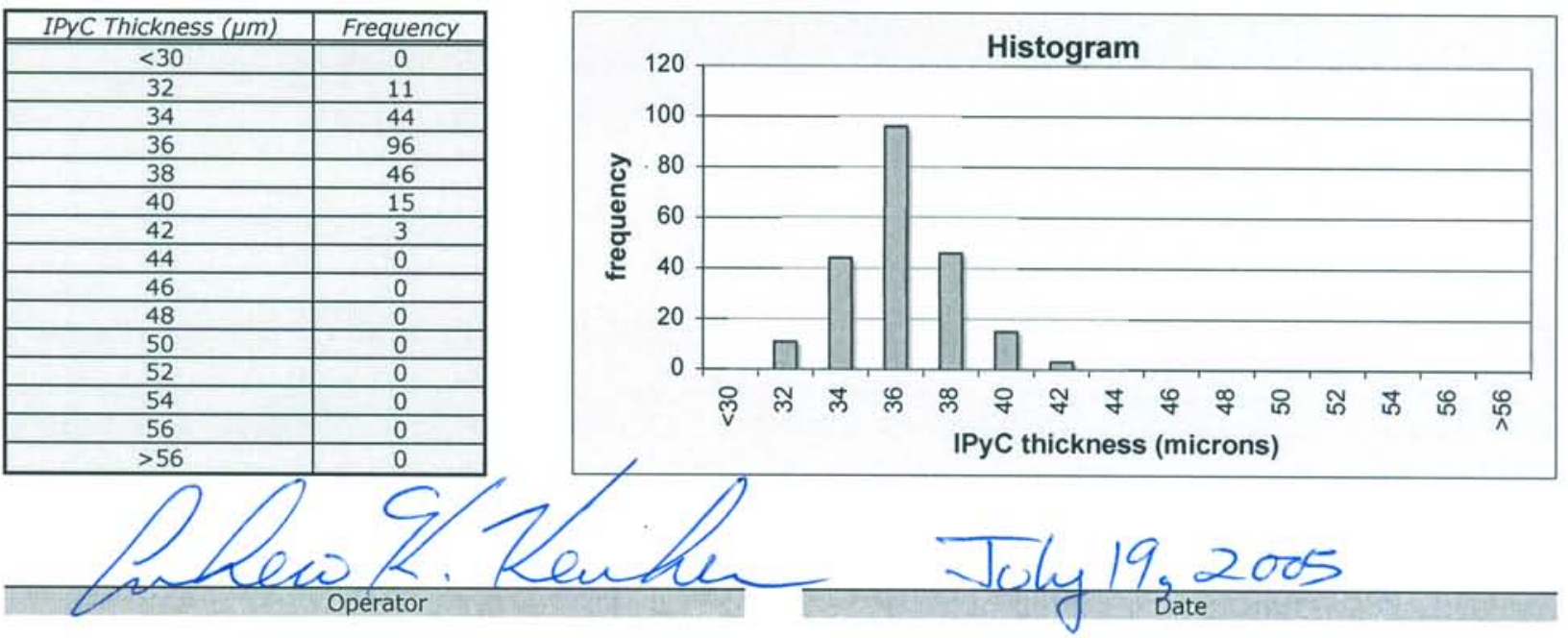


Data Report Form DRF-08: Imaging of Coated Particle Cross-sections Using an Optical Microscope System

\begin{tabular}{|r|l|}
\hline Procedure: & AGR-CHAR-DAM-08 Rev. 0 \\
\hline Operator: & Andrew K. Kercher \\
\hline Sample ID: & NUCO350-29BI-C \\
\hline Sample Description: & IPyC/Buffer on BWXT kernel composite 69300 \\
\hline Mount Number(s): & M05070101 \\
\hline Folder name containing images: & \Imc-agr\AGR\ImageProcessing \P05070601\} $\\
{\hline}$
\end{tabular}

DMR Calibration Expiration Date: $3 / 24 / 06$

Stage Micrometer Calibration Expiration Date: $2 / 17 / 07$

Measured Value for $500 \mu \mathrm{m}$ in Stage Micrometer Image: $499.6 \mu \mathrm{m}$
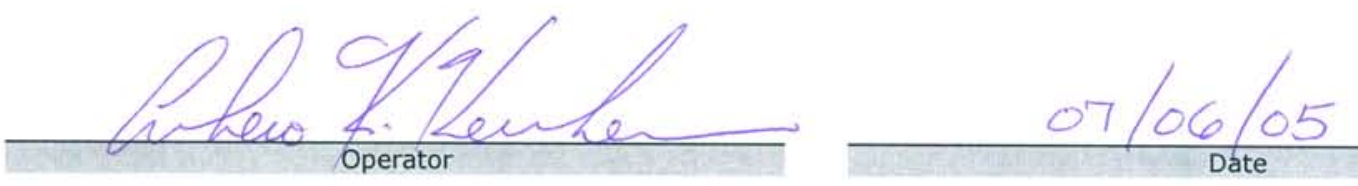
Data Report Form DRF-11A: Measurement of Buffer Layer Thickness

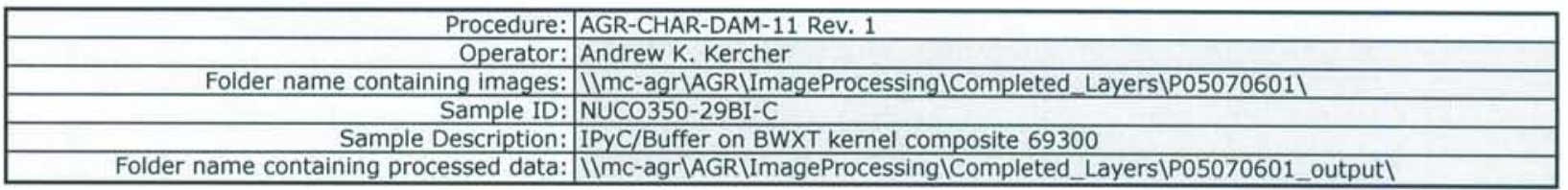

\begin{tabular}{r|r|}
\hline Number of buffer layers analyzed: & 120 \\
\hline Mean of the average buffer thickness of each particle $(\mu \mathrm{m}):$ & 107.3 \\
\hline Standard deviation in the average buffer thickness of each particle $(\mu \mathrm{m}):$ & 8.0 \\
\hline
\end{tabular}

Distribution of the average buffer layer thickness (top binned)

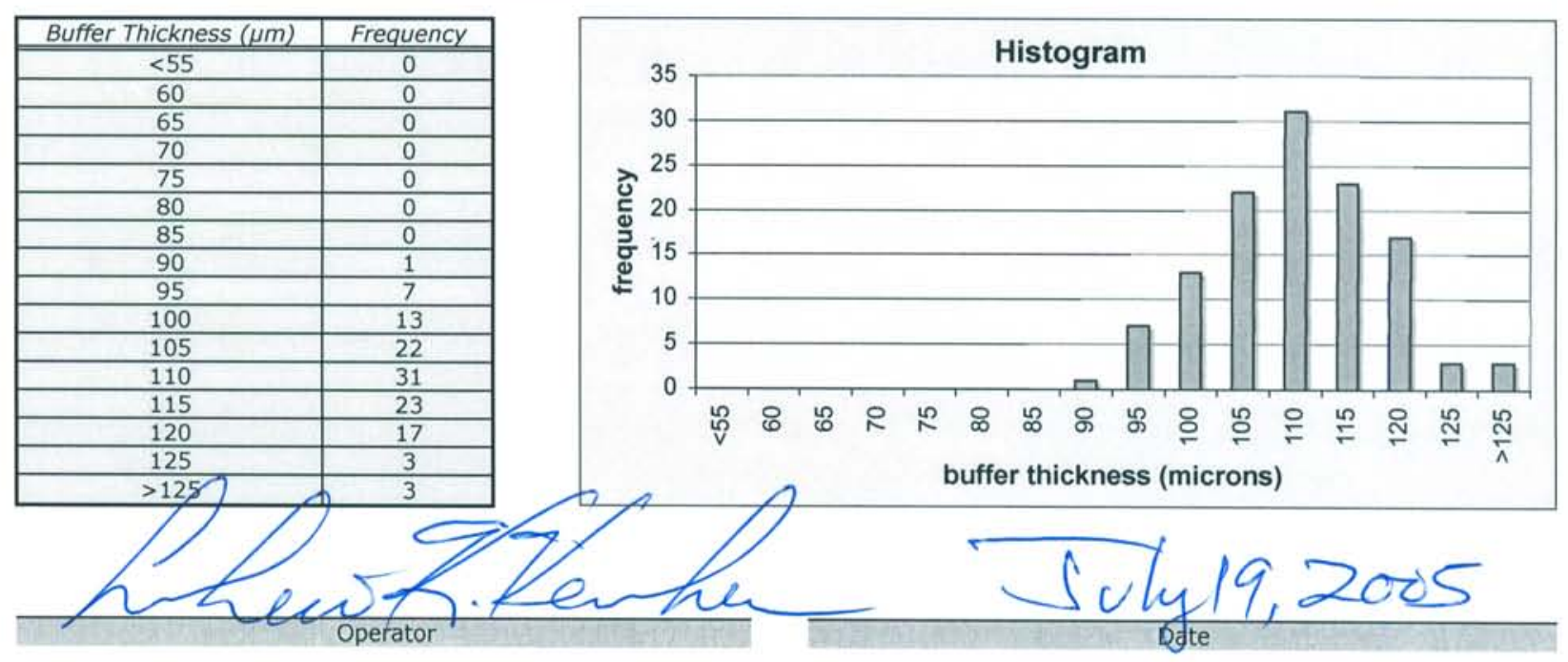


Data Report Form DRF-11B; Measurement of Inner Pyrocarbon Layer Thickness

\begin{tabular}{|c|c|}
\hline Procedure: & AGR-CHAR-DAM-11 Rev. 1 \\
\hline Operator: & Andrew K. Kercher \\
\hline Folder name containing images: & 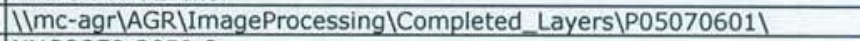 \\
\hline Sample ID: & NUCO350-29BI-C \\
\hline Sample Description: & IPyC/Buffer on BWXT kernel composite 69300 \\
\hline Folder name containing processed data: & IImc-agr|AGR\ImageProcessing\Completed_Layers\P05070601_outpu \\
\hline
\end{tabular}

\begin{tabular}{|r|r|r|}
\hline Number of inner pyrocarbon layers analyzed: & 162 \\
\hline Mean of the average IPyC thickness of each particle $(\mu \mathrm{m}):$ & 33.9 \\
\hline Standard deviation in the average IPyC thickness of each particle $(\mu \mathrm{m}):$ & 2.0 \\
\hline
\end{tabular}

\section{Distribution of the average IPyC layer thickness (top binned)}

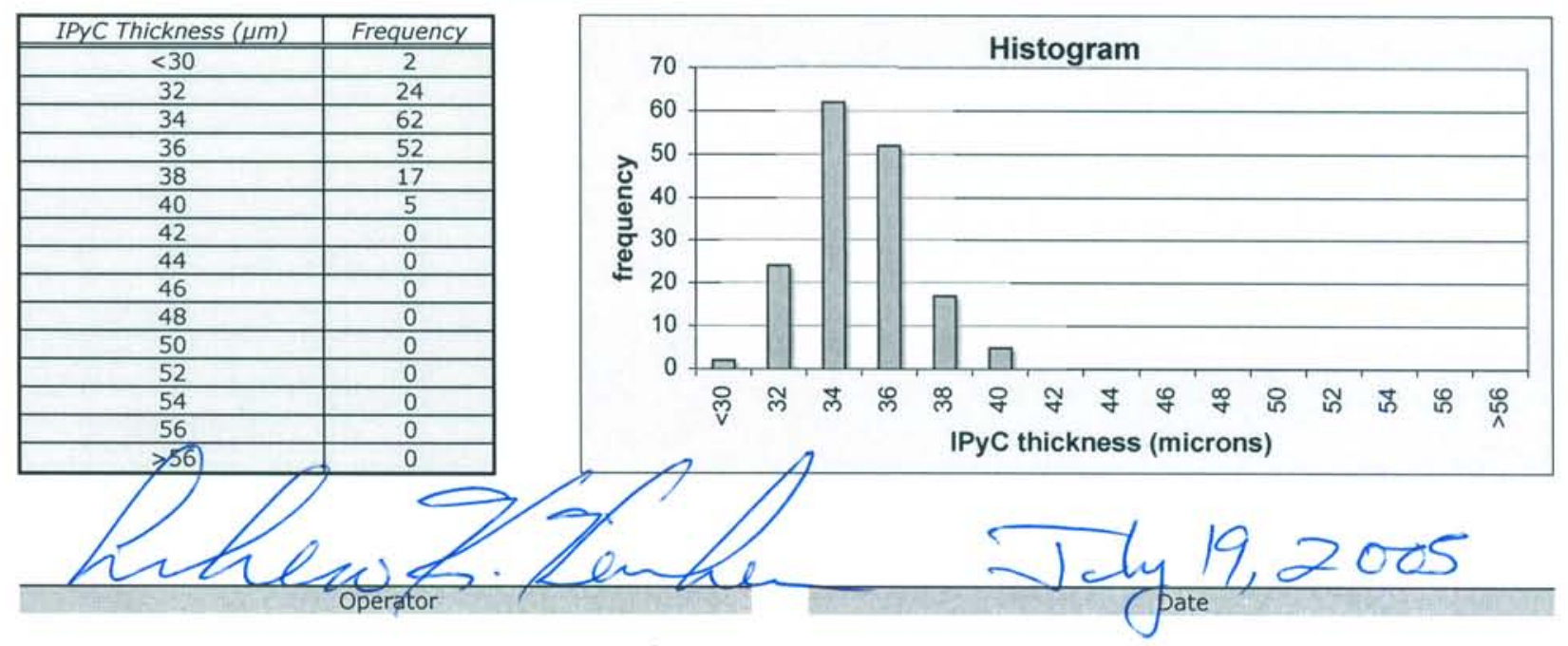


Data Report Form DRF-08: Imaging of Coated Particle Cross-sections Using an Optical Microscope System

\begin{tabular}{|r|l|}
\hline Procedure: & AGR-CHAR-DAM-08 Rev. 0 \\
\hline Operator: & Andrew K. Kercher \\
\hline Sample ID: & NUCO350-66BI-C \\
\hline Sample Description: & Composite (30BI+37BI+29BI) IPyC/Buffer on BWXT composite 69300 \\
\hline Mount Number(s): & M05070601 \\
\hline Folder name containing images: & \Imc-agr\AGR\ImageProcessing\P05070602\} $\\
{\hline}$
\end{tabular}

DMR Calibration Expiration Date: $3 / 24 / 06$

Stage Micrometer Calibration Expiration Date: $2 / 17 / 07$

Measured Value for $500 \mu \mathrm{m}$ in Stage Micrometer Image: $499.6 \mu \mathrm{m}$

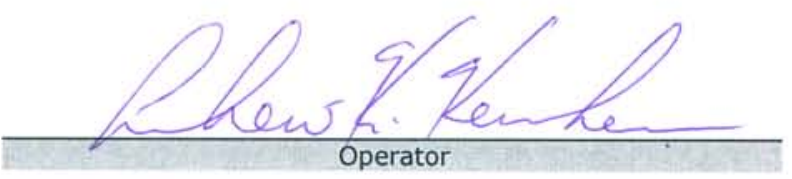

$$
07 / 06 / 05
$$


Data Report Form DRF-11A: Measurement of Buffer Layer Thickness

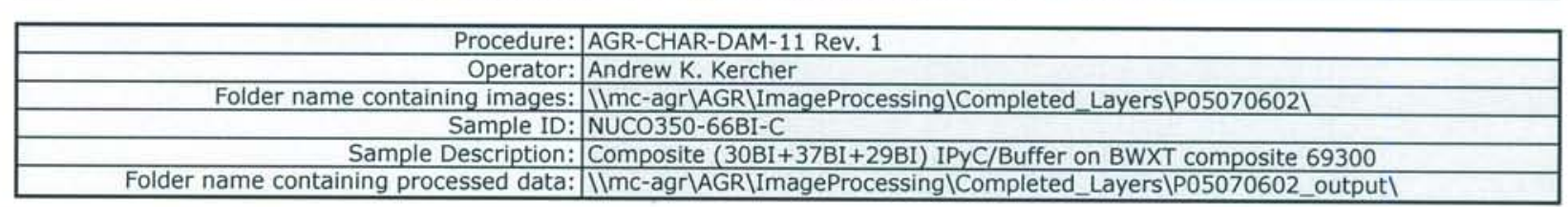

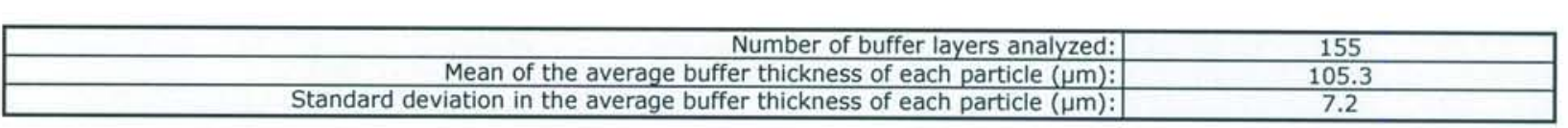

Distribution of the average buffer layer thickness (top binned)

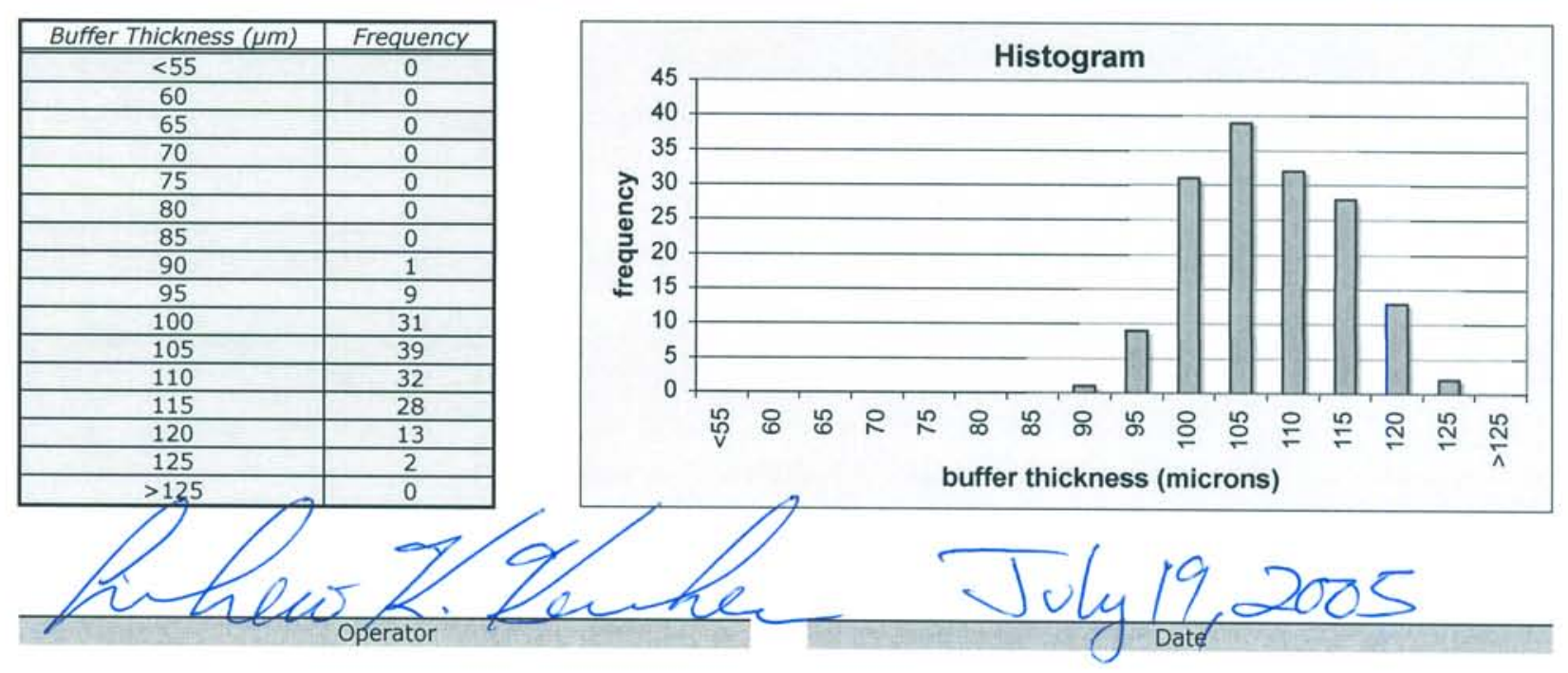




\section{Data Report Form DRF-11B: Measurement of Inner Pyrocarbon Layer Thickness}

\begin{tabular}{|c|c|}
\hline Procedure: & AGR-CHAR-DAM-11 Rev. 1 \\
\hline Operator: & Andrew K. Kercher \\
\hline Folder name containing images: & IImc-agr|AGR\ImageProcessing|Completed_Layers\P05070602\} $\\
{\hline \text { Sample ID: }} &{\text { NUCO350-66BI-C }} \\
{\hline \text { Sample Description: }} &{\text { Composite (30BI+37BI+29BI) IPyC/Buffer on BWXT composite } 69300} \\
{\hline \text { Folder name containing processed data: }} &{\text { IImc-agr|AGR\ImageProcessing|Completed_Layers\P05070602_output }} \\
$\hline
\end{tabular}

Number of inner pyrocarbon layers analyzed: $\quad 193$

Mean of the average IPyC thickness of each particle $(\mu \mathrm{m}):$ : $\quad 34.5$

Standard deviation in the average IPyC thickness of each particle $(\mu \mathrm{m})$

2.2

\section{Distribution of the average IPyC layer thickness (top binned)}

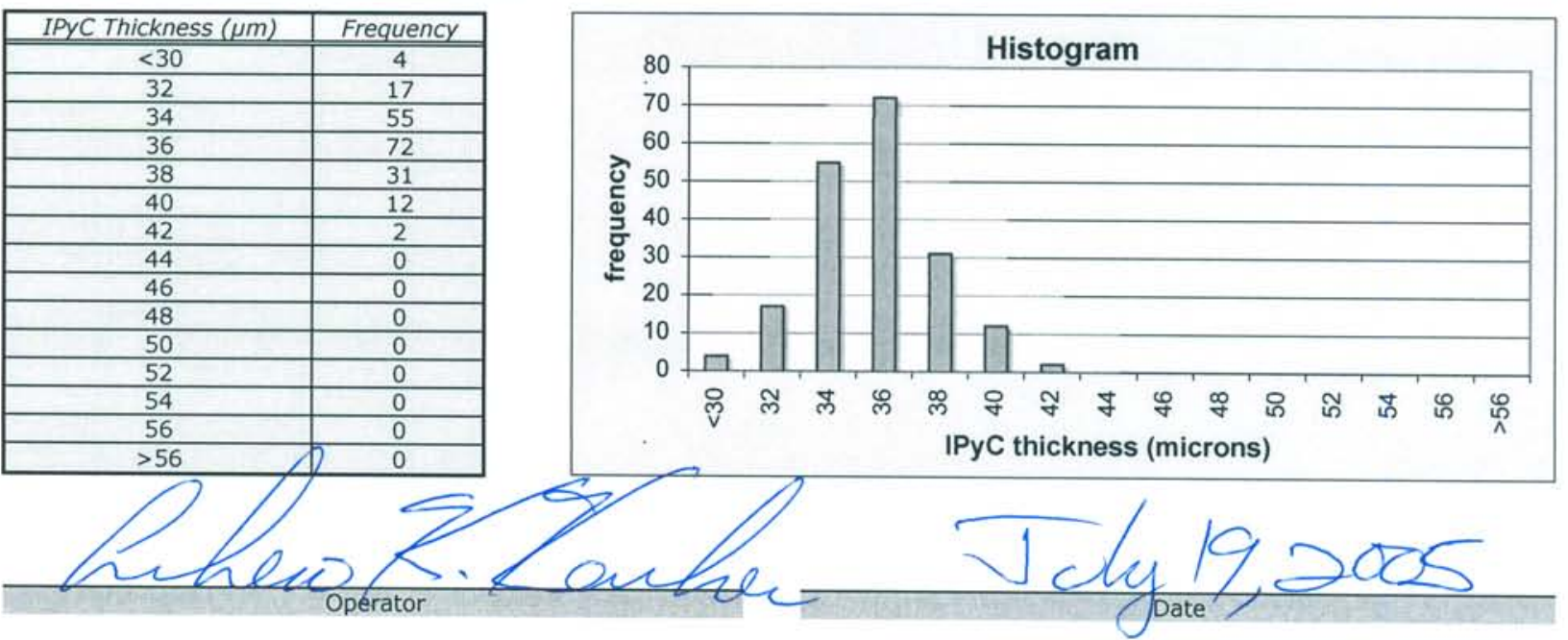


Data Report Form DRF-22: Estimation of Average Particle Weight

\begin{tabular}{|r|l|}
\hline Procedure: & AGR-CHAR-DAM-22 Rev. 1 \\
\hline Operator: & Dixie Barker \\
\hline Particle Lot ID: & NUCO350-66BI \\
\hline Particle Lot Description: & Composite (30BI+37BI+29BI) IPyC/Buffer on BWXT 69300 \\
\hline Filename: & IImc-agr|AGRIParticleWeight/W05072101 DRF22R1.xIs \\
\hline
\end{tabular}

\begin{tabular}{|r|c|c|c|c|c|}
\hline & Sample 1 & Sample 2 & Sample 3 & Sample 4 & Sample 5 \\
\hline Weight of particles (g): & $7.92 \mathrm{E}-02$ & $9.73 \mathrm{E}-02$ & $8.60 \mathrm{E}-02$ & $8.78 \mathrm{E}-02$ & $7.42 \mathrm{E}-02$ \\
\hline Number of particles: & 201 & 246 & 218 & 222 & 188 \\
\hline Average weight/particle (g): & $3.94 \mathrm{E}-04$ & $3.96 \mathrm{E}-04$ & $3.94 \mathrm{E}-04$ & $3.95 \mathrm{E}-04$ & $3.95 \mathrm{E}-04$ \\
\hline
\end{tabular}

Mean average weight/particle $(\mathrm{g}): 3.95 \mathrm{E}-04$

Uncertainty in mean average weight/particle $(g): 2.92 \mathrm{E}-07$
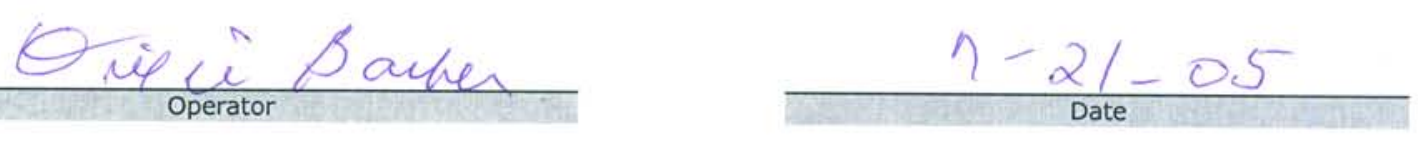
Data Report Form DRF-31: Measurement of Open Porosity using a Mercury Porosimeter

\begin{tabular}{|c|c|}
\hline Procedure: & AGR-CHAR-DAM-31 Rev. 0 \\
\hline Operator: & S. D. Nunn \\
\hline Coated particle batch ID: & NUCO350-66BI-E \\
\hline Batch Description: & IPyC coated BWXT NUCO composite 69300 \\
\hline Thermocouple Expiration Date: & $5 / 23 / 06$ \\
\hline Penetrometer Expiration Date: & $5 / 25 / 06$ \\
\hline Completed DRF Filename: & \Imc-agr|AGR\Porosimeter|S05071402\S05071402_DRF31R0.xIS \\
\hline
\end{tabular}

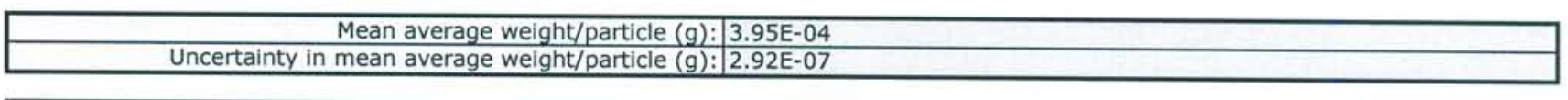

\begin{tabular}{|r|l|}
\hline Weight of particles $(\mathrm{g}):$ & 4.1698 \\
\hline Approximate number of particles: & 10556 \\
\hline Uncertainty in number of particles: & 8 \\
\hline Total envelope volume of sample (cc): & 1.391 \\
\hline Average envelope volume/particle (cc): & $1.32 \mathrm{E}-04$ \\
\hline Sample envelope density $(\mathrm{g} / \mathrm{cc}):$ & 2.998 \\
\hline
\end{tabular}

\begin{tabular}{|r|l|}
\hline Average particle diameter (microns): & $6.31 \mathrm{E}+02$ \\
\hline Average surface area/particle $(\mathrm{cm} 2):$ & $1.25 \mathrm{E}-02$ \\
\hline Total sample surface area $(\mathrm{cm} 2):$ & $1.32 \mathrm{E}+02$ \\
\hline Open porosity $(\mathrm{ml} / \mathrm{m} 2):$ & $1.18 \mathrm{E}-02$ \\
\hline Intruded mercury volume from $250-10,000$ \\
\hline
\end{tabular}
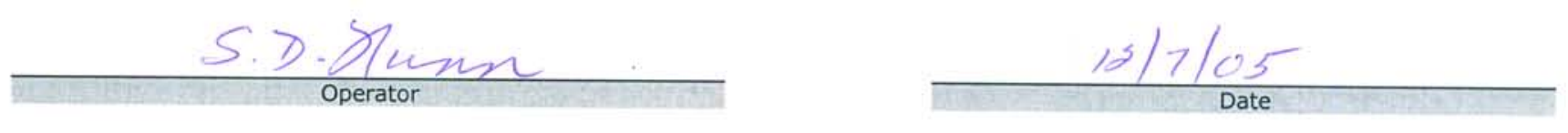
Data Report Form DRF-22: Estimation of Average Particle Weight

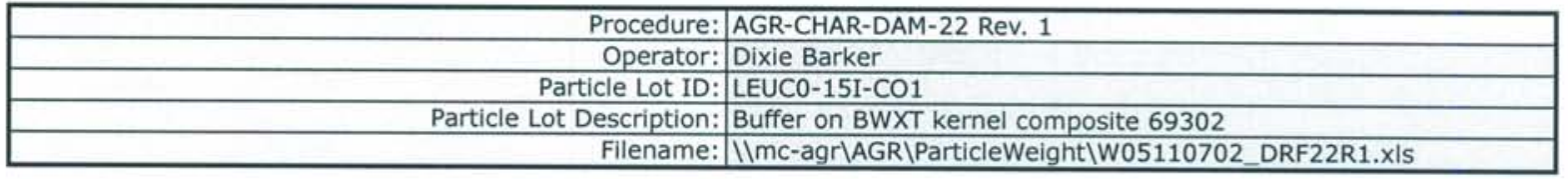

\begin{tabular}{|r|c|c|c|c|c|}
\hline & Sample 1 & Sample 2 & Sample 3 & Sample 4 & Sample 5 \\
\hline Weight of particles (g): & $6.97 \mathrm{E}-02$ & $6.15 \mathrm{E}-02$ & $5.69 \mathrm{E}-02$ & $5.93 \mathrm{E}-02$ & $6.72 \mathrm{E}-02$ \\
\hline Number of particles: & 163 & 144 & 134 & 139 & 159 \\
\hline Average weight/particle (g): & $4.28 \mathrm{E}-04$ & $4.27 \mathrm{E}-04$ & $4.25 \mathrm{E}-04$ & $4.27 \mathrm{E}-04$ & $4.23 \mathrm{E}-04$ \\
\hline
\end{tabular}

Mean average weight/particle $(\mathrm{g}): 4.26 \mathrm{E}-04$

Uncertainty in mean average weight/particle $(\mathrm{g}): 9.19 \mathrm{E}-07$
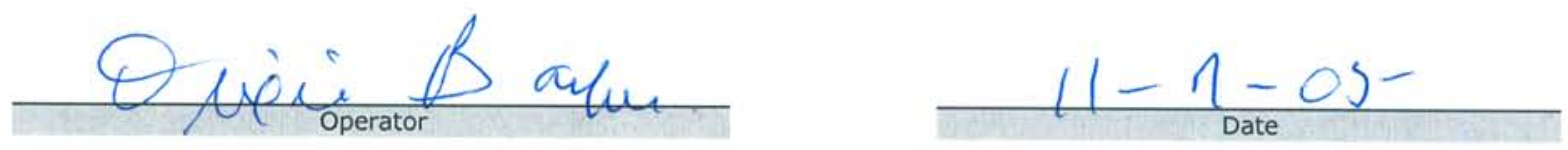
Data Report Form DRF-31: Measurement of Open Porosity using a Mercury Porosimeter

\begin{tabular}{|c|c|}
\hline Procedure: & AGR-CHAR-DAM-31 Rev. 0 \\
\hline Operator: & S. D. Nunn \\
\hline Coated particle batch ID: & LEU01-15I-D01 \\
\hline Batch Description: & IPyC coated BWXT composite 69302 \\
\hline Thermocouple Expiration Date: & $5 / 23 / 06$ \\
\hline Penetrometer Expiration Date: & $5 / 25 / 06$ \\
\hline Completed DRF Filename: & |Imc-agr|AGR\Porosimeter|S05110801|S05110801_DRF31R0.xIs \\
\hline
\end{tabular}

Mean average weight/particle $(g): 4.26 \mathrm{E}-04$

Uncertainty in mean average weight/particle $(\mathrm{g}): 9.19 \mathrm{E}-07$

\begin{tabular}{|l|l|}
\hline Weight of particles $(\mathrm{g}):$ & 4.0001 \\
\hline Approximate number of particles: & 9390 \\
\hline Uncertainty in number of particles: & 20 \\
\hline Total envelope volume of sample $(\mathrm{cc}):$ & 1.344 \\
\hline Average envelope volume/particle $(\mathrm{cc}):$ & $1.43 \mathrm{E}-04$ \\
\hline Sample envelope density $(\mathrm{g} / \mathrm{cc}):$ & 2.976 \\
\hline Average particle diameter (microns): & $6.49 \mathrm{E}+02$ \\
\hline Average surface area/particle $(\mathrm{cm} 2):$ & $1.32 \mathrm{E}-02$ \\
\hline Total sample surface area $(\mathrm{cm} 2):$ & $1.24 \mathrm{E}+02$ \\
\hline Open porosity $(\mathrm{ml} / \mathrm{m} 2):$ & $1.93 \mathrm{E}-02$ \\
\hline
\end{tabular}




\section{Characterization of IPyC on additional interrupted batches}

This section reports results not required by the fuel specification and is provided for information only.

An additional three batches of particles were removed from the coater after deposition of only the buffer and IPyC layers. These particles contained LEUCO kernels and were fabricated for use in the production of TRISO particles where the coating was interrupted between the IPyC and $\mathrm{SiC}$ deposition steps. This section contains data on the inner pyrocarbon (IPyC) coating density and other properties for these particles. The data was obtained according to product inspection plan AGR-CHAR-PIP-02R2 with the exception that no other samples were riffled from the batch other than the one for the IPyC density measurement. The IPyC on these particles was deposited under similar conditions as the IPyC on the particles in composite LEU01-46T and should therefore be representative of the IPyC on the particles in that composite. The results of these measurements are included here because they provide further confirmation to the results in section 9 of the acceptability of the IPyC deposition process for IPyC density. These additional results are valuable because they are for coatings on the same LEUCO kernel composite as that used for LEU01-46T and they were obtained with a newer revision of the data acquisition method for IPyC density measurement which called for measurement on a greater number of coating fragments for improved sampling statistics.

The following page shows the inspection report form (IRF-02B). Following IRF-02B are the individual data report forms for the measurements that were performed. 
ORNL/TM-2006/019, Rev. 1

\section{Inspection Report Form IRF-02B: Interrupted Coating Batches - IPYC Density}

Procedure: AGR-CHAR-PIP-02 Rev. 4

Batch 1 ID: LEU01-23I

Batch 1 description: Variant 3b: IPyC/Buffer on BWXT kernel composite 69302

Batch 2 ID: LEU01-26I

Batch 2 description: Variant 3b: IPyC/Buffer on BWXT kernel composite 69302

Batch 3 ID: LEU01-41I

Batch 3 description: Variant 3b: IPyC/Buffer on BWXT kernel composite 69302

\begin{tabular}{|c|c|c|c|c|c|c|c|c|c|}
\hline \multirow[b]{2}{*}{ Property } & \multicolumn{4}{|c|}{ Measured Data } & \multirow{2}{*}{\begin{tabular}{|c|} 
Specification \\
INL EDF-4380 \\
Rev. 6 \\
\end{tabular}} & \multirow[b]{2}{*}{ Acceptance Criteria } & \multirow{2}{*}{$\begin{array}{l}\text { Acceptance } \\
\text { Test Value }\end{array}$} & \multirow{2}{*}{$\begin{array}{c}\text { Pass } \\
\text { or } \\
\text { fail }\end{array}$} & \multirow{2}{*}{$\begin{array}{c}\text { Data } \\
\text { Records }\end{array}$} \\
\hline & $\begin{array}{l}\text { Mean } \\
(\mathrm{x})\end{array}$ & \begin{tabular}{|c|} 
Std. Dev. \\
(s)
\end{tabular} & $\begin{array}{c}\text { \# measured } \\
(n)\end{array}$ & $\begin{array}{l}k \text { or } t \\
\text { value }\end{array}$ & & & & & \\
\hline \multirow{4}{*}{ Batch 1: IPyC sink/float density $\left(\mathrm{Mg} / \mathrm{m}^{3}\right)$} & \multirow{4}{*}{1.9056} & \multirow{4}{*}{0.0127} & \multirow{4}{*}{48} & \multirow{2}{*}{1.678} & \multirow{2}{*}{$\begin{array}{c}\text { mean } \\
1.90 \pm 0.05\end{array}$} & $A=x-t s / \sqrt{ } n \geq 1.85$ & 1.903 & pass & \multirow{4}{*}{ DRF-03 } \\
\hline & & & & & & $B=x+t s / \sqrt{ } n \leq 1.95$ & 1.909 & pass & \\
\hline & & & & \multirow{2}{*}{2.868} & \multirow{2}{*}{$\begin{array}{c}\text { dispersion } \\
\leq 0.01 \leq 1.80 \\
\leq 0.01 \geq 2.00 \\
\end{array}$} & $C=x-k s>1.80$ & 1.869 & pass & \\
\hline & & & & & & $D=x+k s<2.00$ & 1.942 & pass & \\
\hline \multirow{3}{*}{ Batch 2: IPyC sink/float density $\left(\mathrm{Mg} / \mathrm{m}^{3}\right)$} & \multirow{3}{*}{1.9016} & \multirow{3}{*}{0.0144} & \multirow{3}{*}{51} & 1.676 & $\begin{array}{c}\text { mean } \\
1.90 \pm 0.05\end{array}$ & $A=x-t s / \sqrt{ } n \geq 1.85$ & 1.898 & pass & \multirow{3}{*}{ DRF-03 } \\
\hline & & & & \multirow{2}{*}{2.848} & \multirow{2}{*}{$\begin{array}{c}\text { dispersion } \\
\leq 0.01 \leq 1.80 \\
\leq 0.01 \geq 2.00\end{array}$} & $C=x-k s>1.80$ & 1.861 & pass & \\
\hline & & & & & & $D=x+k s<2.00$ & 1.943 & pass & \\
\hline \multirow{4}{*}{ Batch 3: IPyC sink/float density $\left(\mathrm{Mg} / \mathrm{m}^{3}\right)$} & \multirow{4}{*}{1.9045} & \multirow{4}{*}{0.0130} & \multirow{4}{*}{46} & \multirow{2}{*}{1.679} & \multirow{2}{*}{$\begin{array}{c}\text { mean } \\
1.90 \pm 0.05\end{array}$} & $A=x-t s / \sqrt{ } n \geq 1.85$ & 1.901 & pass & \multirow{4}{*}{ DRF-03 } \\
\hline & & & & & & $B=x+t s / \sqrt{ } n \leq 1.95$ & 1.908 & pass & \\
\hline & & & & \multirow{2}{*}{2.882} & \multirow{2}{*}{$\begin{array}{c}\text { dispersion } \\
\leq 0.01 \leq 1.80 \\
\leq 0.01 \geq 2.00\end{array}$} & $C=x-k s>1.80$ & 1.867 & pass & \\
\hline & & & & & & $D=x+k s<2.00$ & 1.942 & pass & \\
\hline
\end{tabular}

Comments

The process conditions for IPYC and Buffer were the same as the AGR-1 baseline process conditions.

These results demostrate that the baseline IPyC process conditions satisfy the IPyC density specification with the exception that they were not obtained prior to baseline fuel manufacture.

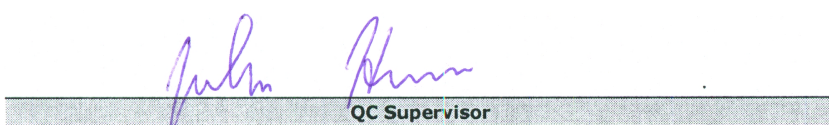

$3-2-06$ 


\begin{tabular}{|r|l|}
\hline Procedure: & AGR-CHAR-DAM-03 Rev. 2 \\
\hline Operator: & Dixie Barker \\
\hline Filename: & IImc-agr\AGR\DensityColumn\D06012501_DRF03R2.xIs \\
\hline Sample ID: & LEU01-23I-B01 \\
\hline Sample description: & Variant 3b: IPyC/Buffer on BWXT kernel composite 69302 \\
\hline Float expiration date: & $07 / 2007$ \\
\hline Gauge expiration date: & $01 / 2007$ \\
\hline Bath temperature: & $22.9^{\circ} \mathrm{C}$ \\
\hline
\end{tabular}

\begin{tabular}{|c|c|c|c|}
\hline \multicolumn{4}{|c|}{ Calibrated Floats } \\
\hline Density & $\begin{array}{l}\text { Top of } \\
\text { Float }\end{array}$ & $\begin{array}{l}\text { Bottom of } \\
\text { Float }\end{array}$ & $\begin{array}{c}\text { Center of } \\
\text { Mass }\end{array}$ \\
\hline 1.800 & 122.58 & 132.72 & 127.65 \\
\hline 1.850 & 223.47 & 231.53 & 227.50 \\
\hline 1.900 & 326.68 & 332.74 & 329.71 \\
\hline 1.950 & 429.77 & 437.46 & 433.62 \\
\hline 2.000 & 535.93 & 543.23 & 539.58 \\
\hline \multicolumn{4}{|c|}{ Linear Fit } \\
\hline slope & StDev & intercept & StDev \\
\hline $4.85 E-04$ & $2.89 E-06$ & $1.74 E+00$ & $9.64 \mathrm{E}-04$ \\
\hline
\end{tabular}

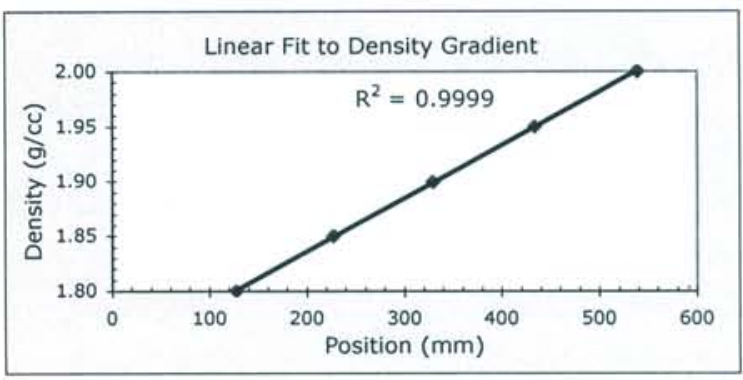

\begin{tabular}{|c|c|c|c|c|c|c|c|c|}
\hline $\begin{array}{c}\text { Fragment } \\
\text { Number }\end{array}$ & $\begin{array}{c}\text { Fragment } \\
\text { Position }\end{array}$ & $\begin{array}{c}\text { Calculated } \\
\text { Density }\end{array}$ & $\begin{array}{c}\text { Fragment } \\
\text { Number }\end{array}$ & $\begin{array}{c}\text { Fragment } \\
\text { Position } \\
\end{array}$ & $\begin{array}{c}\text { Calculated } \\
\text { Density }\end{array}$ & $\begin{array}{c}\text { Fragment } \\
\text { Number }\end{array}$ & $\begin{array}{c}\text { Fragment } \\
\text { Position }\end{array}$ & $\begin{array}{c}\text { Calculated } \\
\text { Density } \\
\end{array}$ \\
\hline 1 & 276.61 & 1.8733 & 26 & 351.30 & 1.9096 & 51 & & \\
\hline 2 & 282.13 & 1.8760 & 27 & 352.03 & 1.9099 & 52 & & \\
\hline 3 & 287.38 & 1.8785 & 28 & 354.21 & 1.9110 & 53 & & \\
\hline 4 & 291.23 & 1.8804 & 29 & 354.85 & 1.9113 & 54 & & \\
\hline 5 & 299.63 & 1.8845 & 30 & 355.47 & 1.9116 & 55 & & \\
\hline 6 & 310.91 & 1.8900 & 31 & 355.47 & 1.9116 & 56 & & \\
\hline 7 & 313.07 & 1.8910 & 32 & 356.37 & 1.9120 & 57 & & \\
\hline 8 & 317.71 & 1.8933 & 33 & 357.52 & 1.9126 & 58 & & \\
\hline 9 & 318.83 & 1.8938 & 34 & 358.02 & 1.9128 & 59 & & \\
\hline 10 & 323.08 & 1.8959 & 35 & 358.34 & 1.9130 & 60 & & \\
\hline 11 & 324.22 & 1.8964 & 36 & 360.37 & 1.9140 & 61 & & \\
\hline 12 & 328.36 & 1.8984 & 37 & 361.54 & 1.9145 & 62 & & \\
\hline 13 & 330.91 & 1.8997 & 38 & 361.54 & 1.9145 & 63 & & \\
\hline 14 & 332.69 & 1.9005 & 39 & 362.17 & 1.9148 & 64 & & \\
\hline 15 & 333.78 & 1.9011 & 40 & 365.31 & 1.9164 & 65 & & \\
\hline 16 & 334.56 & 1.9014 & 41 & 366.52 & 1.9169 & 66 & & \\
\hline 17 & 337.37 & 1.9028 & 42 & 368.35 & 1.9178 & 67 & & \\
\hline 18 & 342.48 & 1.9053 & 43 & 370.63 & 1.9189 & 68 & & \\
\hline 19 & 343.07 & 1.9056 & 44 & 373.61 & 1.9204 & 69 & & \\
\hline 20 & 345.44 & 1.9067 & 45 & 377.86 & 1.9224 & 70 & & \\
\hline 21 & 345.97 & 1.9070 & 46 & 379.11 & 1.9231 & 71 & & \\
\hline 22 & 346.79 & 1.9074 & 47 & 380.42 & 1.9237 & 72 & & \\
\hline 23 & 347.45 & 1.9077 & 48 & 381.00 & 1.9240 & 73 & & \\
\hline 24 & 348.31 & 1.9081 & 49 & & & 74 & & \\
\hline 25 & 350.73 & 1.9093 & 50 & & & 75 & & \\
\hline \multicolumn{4}{|c|}{ Average density of PyC fragments: } & \multicolumn{5}{|c|}{1.9056} \\
\hline \multirow{2}{*}{\multicolumn{4}{|c|}{$\begin{array}{r}\text { Standard deviation in density of PyC fragments: } \\
\text { Uncertainty in calculated density of PyC fragments: }\end{array}$}} & \multicolumn{5}{|c|}{0.0127} \\
\hline & & & & \multicolumn{5}{|c|}{0.0015} \\
\hline
\end{tabular}

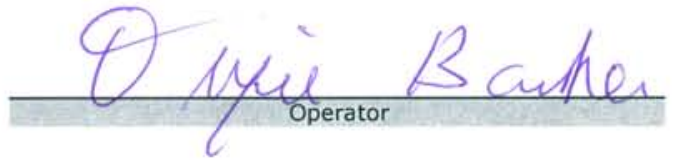

$$
1-25-06
$$


Data Report Form DRF-03: Measurement of PyC Density using a Density Gradient Column

\begin{tabular}{|r|l|}
\hline Procedure: & AGR-CHAR-DAM-03 Rev. 2 \\
\hline Operator: & Dixie Barker \\
\hline Filename: & IImc-agr\AGR\DensityColumn\D06012601_DRF03R2.xIs \\
\hline Sample ID: & LEU01-26I-B01 \\
\hline Sample description: & Variant 3b: IPyC/Buffer on BWXT kernel composite 69302 \\
\hline Float expiration date: & $07 / 2007$ \\
\hline Gauge expiration date: & $01 / 2007$ \\
\hline Bath temperature: & $22.5^{\circ} \mathrm{C}$ \\
\hline
\end{tabular}

\begin{tabular}{|c|c|c|c|}
\hline \multicolumn{4}{|c|}{ Calibrated Floats } \\
\hline Density & $\begin{array}{c}\text { Top of } \\
\text { Float }\end{array}$ & $\begin{array}{c}\text { Bottom of } \\
\text { Float }\end{array}$ & $\begin{array}{c}\text { Center of } \\
\text { Mass }\end{array}$ \\
\hline \hline 1.800 & 128.13 & 136.87 & 132.50 \\
\hline 1.850 & 228.36 & 236.83 & 232.60 \\
\hline 1.900 & 333.25 & 338.84 & 336.05 \\
\hline 1.950 & 435.49 & 443.49 & 439.49 \\
\hline 2.000 & 541.90 & 548.97 & 545.44 \\
\hline
\end{tabular}

\begin{tabular}{|c|c|c|c|}
\hline \multicolumn{4}{|c|}{ Linear Fit } \\
\hline slope & StDev & intercept & StDev \\
\hline \hline $4.84 \mathrm{E}-04$ & $2.90 \mathrm{E}-06$ & $1.74 \mathrm{E}+00$ & $9.84 \mathrm{E}-04$ \\
\hline
\end{tabular}

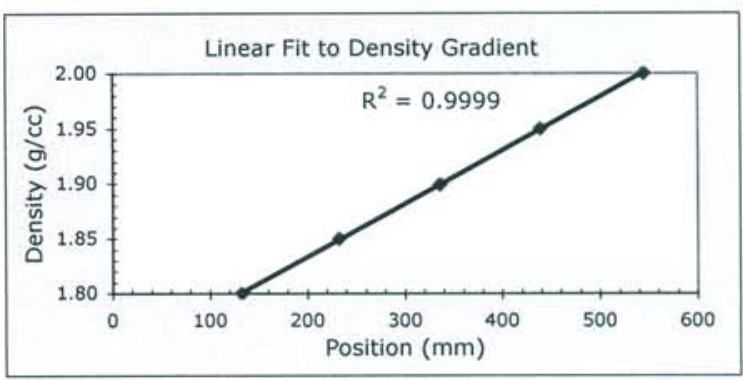

\begin{tabular}{|c|c|c|c|c|c|c|c|c|}
\hline \multicolumn{9}{|c|}{ Sample Density } \\
\hline $\begin{array}{l}\text { Fragment } \\
\text { Number }\end{array}$ & $\begin{array}{c}\text { Fragment } \\
\text { Position }\end{array}$ & $\begin{array}{c}\text { Calculated } \\
\text { Density }\end{array}$ & \begin{tabular}{c|}
$\begin{array}{c}\text { Fragment } \\
\text { Number }\end{array}$ \\
\end{tabular} & $\begin{array}{c}\text { Fragment } \\
\text { Position }\end{array}$ & $\begin{array}{c}\text { Calculated } \\
\text { Density }\end{array}$ & $\begin{array}{c}\text { Fragment } \\
\text { Number }\end{array}$ & $\begin{array}{c}\text { Fragment } \\
\text { Position } \\
\end{array}$ & $\begin{array}{c}\text { Calculated } \\
\text { Density }\end{array}$ \\
\hline 1 & 266.79 & 1.8659 & 26 & 346.54 & 1.9045 & 51 & 393.01 & 1.9270 \\
\hline 2 & 285.87 & 1.8751 & 27 & 346.54 & 1.9045 & 52 & & \\
\hline 3 & 288.11 & 1.8762 & 28 & 347.31 & 1.9049 & 53 & & \\
\hline 4 & 288.60 & 1.8765 & 29 & 350.49 & 1.9064 & 54 & & \\
\hline 5 & 292.72 & 1.8785 & 30 & 351.77 & 1.9070 & 55 & & \\
\hline 6 & 298.39 & 1.8812 & 31 & 353.96 & 1.9081 & 56 & & \\
\hline 7 & 300.12 & 1.8820 & 32 & 353.96 & 1.9081 & 57 & & \\
\hline 8 & 301.41 & 1.8827 & 33 & 355.69 & 1.9089 & 58 & & \\
\hline 9 & 305.86 & 1.8848 & 34 & 356.71 & 1.9094 & 59 & & \\
\hline 10 & 313.26 & 1.8884 & 35 & 357.96 & 1.9100 & 60 & & \\
\hline 11 & 317.17 & 1.8903 & 36 & 360.26 & 1.9112 & 61 & & \\
\hline 12 & 318.36 & 1.8909 & 37 & 361.24 & 1.9116 & 62 & & \\
\hline 13 & 319.60 & 1.8915 & 38 & 362.33 & 1.9122 & 63 & & \\
\hline 14 & 320.91 & 1.8921 & 39 & 363.79 & 1.9129 & 64 & & \\
\hline 15 & 322.75 & 1.8930 & 40 & 363.79 & 1.9129 & 65 & & \\
\hline 16 & 323.99 & 1.8936 & 41 & 365.03 & 1.9135 & 66 & & \\
\hline 17 & 326.39 & 1.8948 & 42 & 365.91 & 1.9139 & 67 & & \\
\hline 18 & 330.64 & 1.8968 & 43 & 366.71 & 1.9143 & 68 & & \\
\hline 19 & 331.75 & 1.8974 & 44 & 371.41 & 1.9166 & 69 & & \\
\hline 20 & 338.46 & 1.9006 & 45 & 372.46 & 1.9171 & 70 & & \\
\hline 21 & 339.67 & 1.9012 & 46 & 375.13 & 1.9184 & 71 & & \\
\hline 22 & 341.79 & 1.9022 & 47 & 379.10 & 1.9203 & 72 & & \\
\hline 23 & 343.23 & 1.9029 & 48 & 380.17 & 1.9208 & 73 & & \\
\hline 24 & 344.00 & 1.9033 & 49 & 380.17 & 1.9208 & 74 & & \\
\hline 25 & 344.93 & 1.9037 & 50 & 383.89 & 1.9226 & 75 & & \\
\hline & Avera & density of $F$ & fragments: & & & 1.9016 & & \\
\hline Star & ard deviation & density of $\mathrm{F}$ & fragments: & & & 0.0144 & & \\
\hline Uncerta & ty in calculat & density of & fragments: & & & 0.0015 & & \\
\hline
\end{tabular}
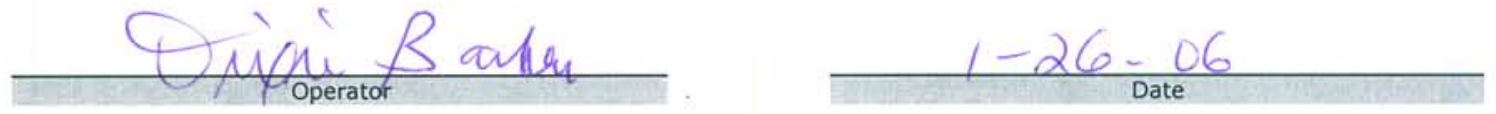
ORNL/TM-2006/019, Rev. 1

Data Report Form DRF-03: Measurement of PyC Density using a Density Gradient Column

\begin{tabular}{|r|l|}
\hline Procedure: & AGR-CHAR-DAM-03 Rev. 2 \\
\hline Operator: & Dixie Barker \\
\hline Filename: & IImc-agr\AGR DensityColumn\D06013001_DRF03R2.xIs \\
\hline Sample ID: & LEU01-41I-B01 \\
\hline Sample description: & Variant 3b: IPyC/Buffer on BWXT kernel composite 69302 \\
\hline Gauge expiration date: & $07 / 2007$ \\
\hline Bath temperature: & $01 / 2007$ \\
\hline
\end{tabular}

\begin{tabular}{|c|c|c|c|}
\hline \multicolumn{4}{|c|}{ Calibrated Floats } \\
\hline Density & $\begin{array}{l}\text { Top of } \\
\text { Float }\end{array}$ & $\begin{array}{l}\text { Bottom of } \\
\text { Float }\end{array}$ & $\begin{array}{c}\text { Center of } \\
\text { Mass }\end{array}$ \\
\hline 1.800 & 127.12 & 136.14 & 131.63 \\
\hline 1.850 & 229.50 & 237.55 & 233.53 \\
\hline 1.900 & 335.50 & 340.95 & 338.23 \\
\hline 1.950 & 439.00 & 446.59 & 442.80 \\
\hline 2.000 & 542.50 & 549.76 & 546.13 \\
\hline \multicolumn{4}{|c|}{ Linear Fit } \\
\hline slope & StDev & intercept & StDev \\
\hline $4.82 \mathrm{E}-04$ & $2.92 E-06$ & $1.74 \mathrm{E}+00$ & $9.95 \mathrm{E}-04$ \\
\hline
\end{tabular}

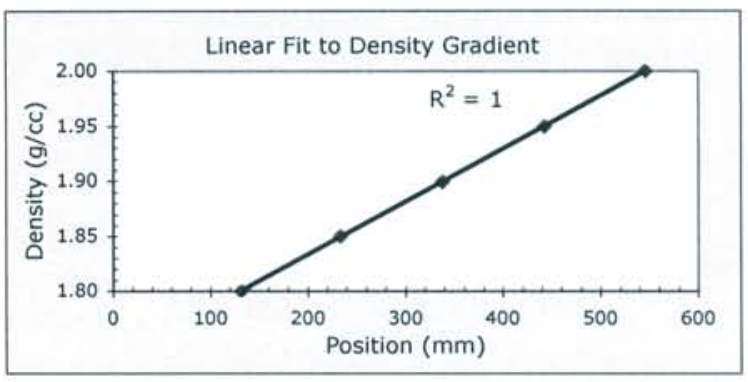

\begin{tabular}{|c|c|c|c|c|c|c|c|c|}
\hline $\begin{array}{l}\text { Fragment } \\
\text { Number }\end{array}$ & $\begin{array}{l}\text { Fragment } \\
\text { Position }\end{array}$ & $\begin{array}{l}\text { Calculated } \\
\text { Density }\end{array}$ & $\begin{array}{l}\text { Fragment } \\
\text { Number }\end{array}$ & $\begin{array}{l}\text { Fragment } \\
\text { Position }\end{array}$ & $\begin{array}{l}\text { Calculated } \\
\text { Density }\end{array}$ & $\begin{array}{l}\text { Fragment } \\
\text { Number }\end{array}$ & $\begin{array}{l}\text { Fragment } \\
\text { Position }\end{array}$ & $\begin{array}{l}\text { Calculated } \\
\text { Density }\end{array}$ \\
\hline 1 & 286.46 & 1.8750 & 26 & 348.83 & 1.9050 & 51 & & \\
\hline 2 & 294.85 & 1.8790 & 27 & 352.30 & 1.9067 & 52 & & \\
\hline 3 & 305.28 & 1.8840 & 28 & 353.32 & 1.9072 & 53 & & \\
\hline 4 & 308.22 & 1.8854 & 29 & 353.81 & 1.9074 & 54 & & \\
\hline 5 & 310.52 & 1.8865 & 30 & 358.69 & 1.9097 & 55 & & \\
\hline 6 & 312.28 & 1.8874 & 31 & 359.79 & 1.9103 & 56 & & \\
\hline 7 & 320.59 & 1.8914 & 32 & 361.05 & 1.9109 & 57 & & \\
\hline 8 & 323.40 & 1.8927 & 33 & 362.21 & 1.9114 & 58 & & \\
\hline 9 & 325.20 & 1.8936 & 34 & 363.30 & 1.9120 & 59 & & \\
\hline 10 & 325.90 & 1.8939 & 35 & 363.30 & 1.9120 & 60 & & \\
\hline 11 & 329.06 & 1.8955 & 36 & 365.45 & 1.9130 & 61 & & \\
\hline 12 & 332.16 & 1.8970 & 37 & 368.29 & 1.9144 & 62 & & \\
\hline 13 & 332.16 & 1.8970 & 38 & 373.79 & 1.9170 & 63 & & \\
\hline 14 & 333.34 & 1.8975 & 39 & 376.08 & 1.9181 & 64 & & \\
\hline 15 & 336.83 & 1.8992 & 40 & 376.49 & 1.9183 & 65 & & \\
\hline 16 & 338.18 & 1.8999 & 41 & 379.22 & 1.9196 & 66 & & \\
\hline 17 & 340.70 & 1.9011 & 42 & 380.71 & 1.9203 & 67 & & \\
\hline 18 & 341.74 & 1.9016 & 43 & 383.98 & 1.9219 & 68 & & \\
\hline 19 & 341.74 & 1.9016 & 44 & 390.28 & 1.9250 & 69 & & \\
\hline 20 & 344.81 & 1.9031 & 45 & 392.92 & 1.9262 & 70 & & \\
\hline 21 & 345.54 & 1.9034 & 46 & 416.92 & 1.9378 & 71 & & \\
\hline 22 & 346.21 & 1.9037 & 47 & & & 72 & & \\
\hline 23 & 346.46 & 1.9039 & 48 & $\div$ & & 73 & & \\
\hline 24 & 346.94 & 1.9041 & 49 & & & 74 & & \\
\hline 25 & 348.16 & 1.9047 & 50 & & & 75 & & \\
\hline \multicolumn{4}{|c|}{ Average density of PyC fragments: } & \multicolumn{5}{|c|}{1.9045} \\
\hline \multirow{2}{*}{\multicolumn{4}{|c|}{$\begin{array}{r}\text { Standard deviation in density of PyC fragments: } \\
\text { Uncertainty in calculated density of PyC fragments: }\end{array}$}} & \multicolumn{5}{|c|}{0.0130} \\
\hline & & & & \multicolumn{5}{|c|}{0.0016} \\
\hline
\end{tabular}
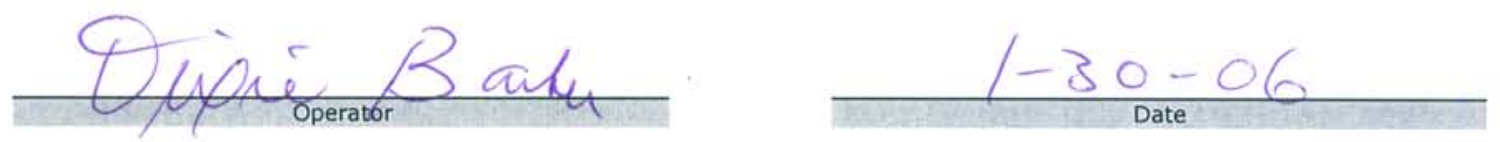
ORNL/TM-2006/019, Rev. 1

Summary of "info only" measurements

Coating Thickness

\begin{tabular}{|llcc|}
\hline & & Buffer thickness $(\mu \mathrm{m})$ & IPyC thickness $(\mu \mathrm{m})$ \\
\cline { 2 - 4 } Batch 1 & LEU01-23I & 109 & 35.2 \\
Batch 2 & LEU01-26I & 108 & 35.1 \\
Batch 3 & LEU01-41I & 110 & 35.0 \\
\hline Averaqe & & 109 & 35.1 \\
\hline
\end{tabular}


ORNL/TM-2006/019, Rev. 1

Data Report Form DRF-08: Imaging of Coated Particle Cross-sections Using an Optical Microscope System

\begin{tabular}{|r|l|}
\hline Procedure: & AGR-CHAR-DAM-08 Rev. 2 \\
\hline Operator: & Andrew K. Kercher \\
\hline Sample ID: & LEU01-23I-B01 \\
\hline Sample description: & Variant 3b: IPyC/Buffer on BWXT kernel composite 69302 \\
\hline Mount ID number: & M06012401L \\
\hline Folder name containing images: & IImc-agr\AGR\ImageProcessing\P06013001\P0601300101\} $\\
{\hline}$
\end{tabular}

DMR calibration expiration date: $9 / 8 / 06$

Calibrated pixels/micron: 2.8260

Stage micrometer calibration expiration date: $2 / 17 / 07$

Measured value for $500 \mu \mathrm{m}$ in stage micrometer image $(\mu \mathrm{m}): 500.0$

\begin{tabular}{|c|c|c|c|}
\hline \multicolumn{4}{|c|}{ Polish-down distance $n, m(\mu \mathrm{m})$} \\
\hline 2,2 & 2,8 & 8,2 & 8,8 \\
\hline \hline 298 & 283 & 297 & 280 \\
\hline
\end{tabular}

\begin{tabular}{|c|c|c|c|c|}
\hline \multicolumn{5}{|c|}{ Approximate layer width in polish plane $(\mu \mathrm{m})$} \\
\hline Kernel radius & Buffer & IPyC & SiC & OPyC \\
\hline \hline 174 & 104 & 36 & & \\
\hline
\end{tabular}

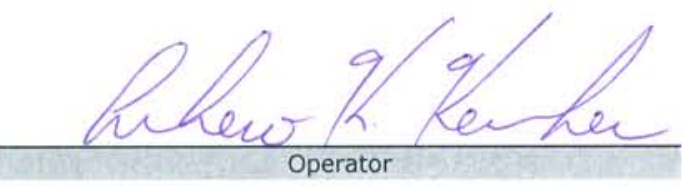

$$
01 / 30 / 06
$$




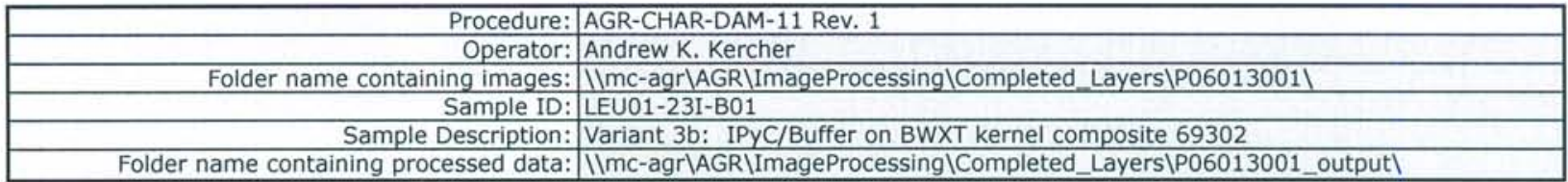

\begin{tabular}{r|c|} 
Number of buffer layers analyzed: & 77 \\
\hline Mean of the average buffer thickness of each particle $(\mu \mathrm{m}):$ & 109.0 \\
\hline Standard deviation in the average buffer thickness of each particle $(\mu \mathrm{m}):$ & 7.4 \\
\hline
\end{tabular}

Distribution of the average buffer layer thickness (top binned)

\begin{tabular}{|c|c|}
\hline Buffer Thickness $(\mu \mathrm{m})$ & Frequency \\
\hline \hline$<55$ & 0 \\
\hline 60 & 0 \\
\hline 65 & 0 \\
\hline 70 & 0 \\
\hline 75 & 0 \\
\hline 80 & 0 \\
\hline 85 & 0 \\
\hline 90 & 6 \\
\hline 95 & 28 \\
\hline 100 & 37 \\
\hline 105 & 58 \\
\hline 110 & 44 \\
\hline 115 & 19 \\
\hline 120 & 7 \\
\hline 125 & 0 \\
\hline$>125$ & 2 \\
\hline
\end{tabular}
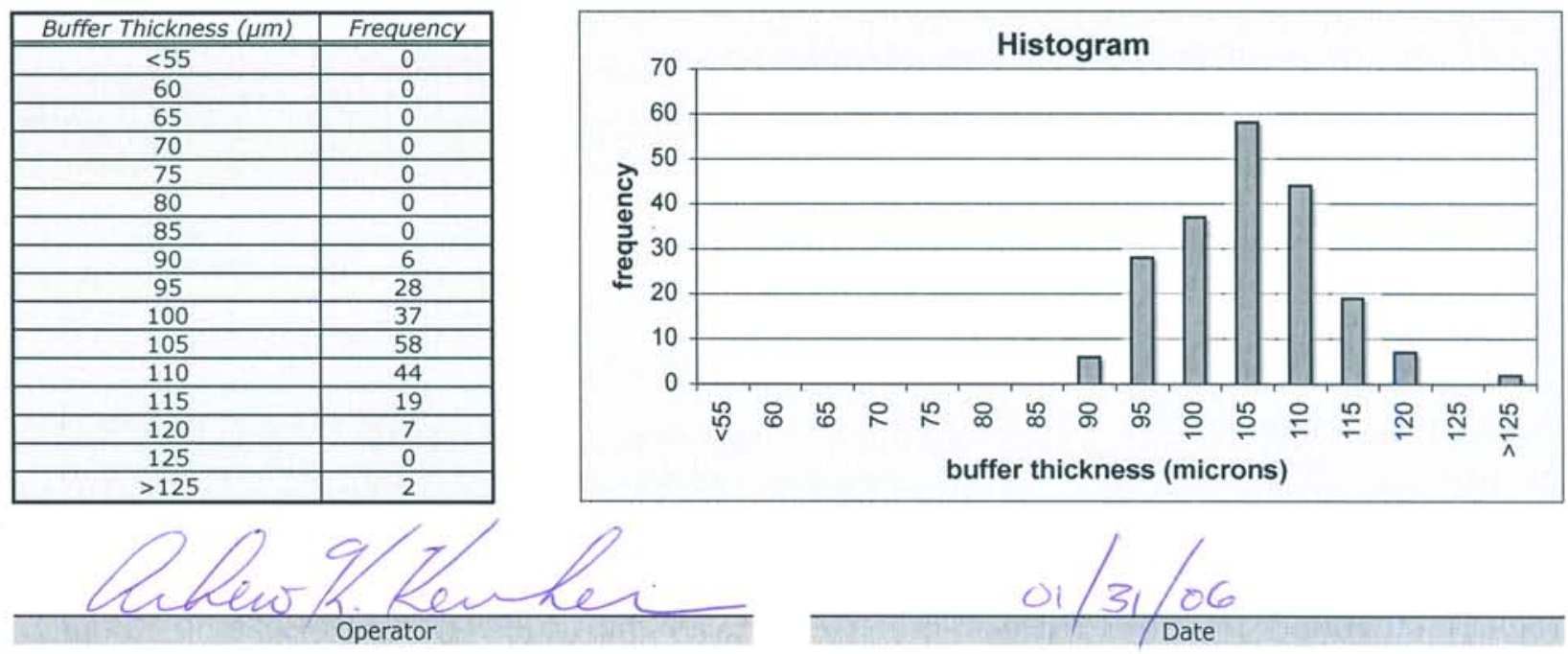
Data Report Form DRF-11B: Measurement of Inner Pyrocarbon Layer Thickness

Procedure: AGR-CHAR-DAM-11 Rev. 1

Operator: Andrew K. Kercher

Folder name containing images: |Imc-agr|AGR\ImageProcessing|Completed_Layers\P06013001

Sample ID: LEU01-23I-B01

Sample Description: Variant 3b: IPyC/Buffer on BWXT kernel composite 69302

Folder name containing processed data: IImc-agr|AGR\ImageProcessing|Completed_Layers|P06013001_output|

Number of inner pyrocarbon layers analyzed: $\quad 80$

Mean of the average IPyC thickness of each particle ( $\mu \mathrm{m})$

Standard deviation in the average IPyC thickness of each particle $(\mu \mathrm{m})$ :

Distribution of the average IPyC layer thickness (top binned)

\begin{tabular}{|c|c|}
\hline IPyC Thickness $(\mu \mathrm{m})$ & Frequency \\
\hline \hline$<30$ & 0 \\
\hline 32 & 0 \\
\hline 34 & 4 \\
\hline 36 & 25 \\
\hline 38 & 53 \\
\hline 40 & 61 \\
\hline 42 & 41 \\
\hline 44 & 12 \\
\hline 46 & 3 \\
\hline 48 & 2 \\
\hline 50 & 0 \\
\hline 52 & 0 \\
\hline 54 & 0 \\
\hline 56 & 0 \\
\hline$>56$ & 0 \\
\hline & \\
\hline
\end{tabular}
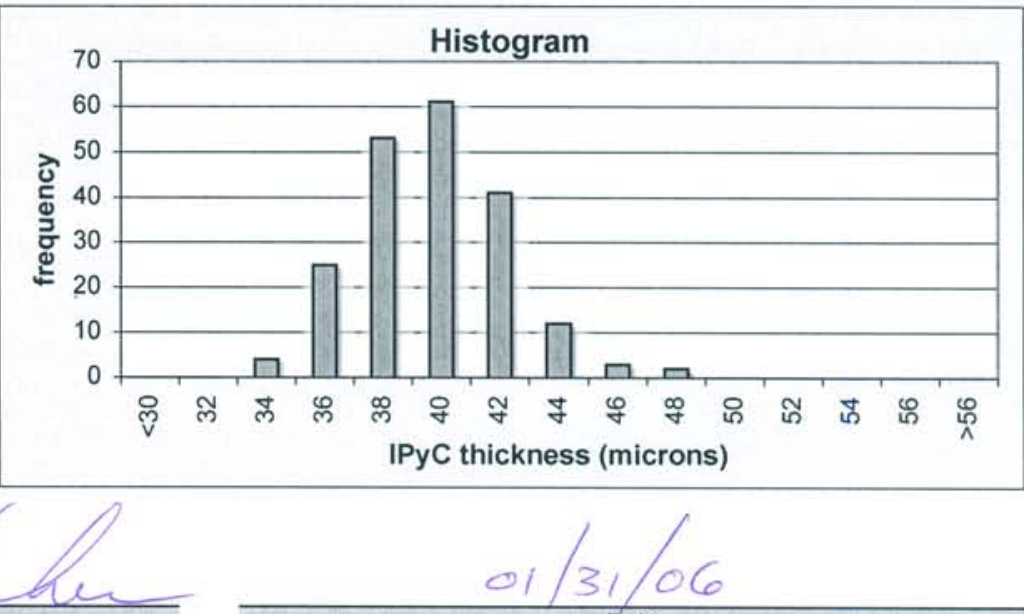

Operator

Date 
ORNL/TM-2006/019, Rev. 1

Data Report Form DRF-08: Imaging of Coated Particle Cross-sections Using an Optical Microscope System

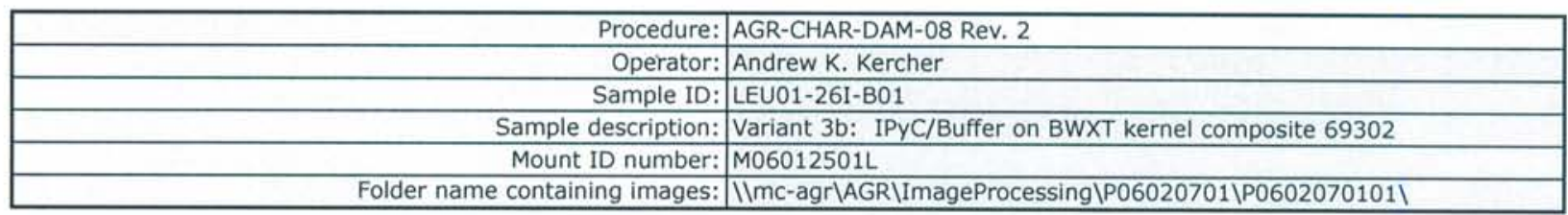

DMR calibration expiration date: $9 / 8 / 06$ Calibrated pixels/micron: 2.8260

Stage micrometer calibration expiration date: $2 / 17 / 07$

Measured value for $500 \mu \mathrm{m}$ in stage micrometer image $(\mu \mathrm{m}): 500.4$

\begin{tabular}{|c|c|c|c|}
\hline \multicolumn{4}{|c|}{ Polish-down distance $n, m(\mu \mathrm{m})$} \\
\hline 2,2 & 2,8 & 8,2 & 8,8 \\
\hline \hline 296 & 303 & 290 & 298 \\
\hline
\end{tabular}

\begin{tabular}{|c|c|c|c|c|}
\hline \multicolumn{5}{|c|}{ Approximate layer width in polish plane $(\mu \mathrm{m})$} \\
\hline Kernel radius & Buffer & IPyC & SiC & OPyC \\
\hline \hline 175 & 100 & 37 & & \\
\hline
\end{tabular}
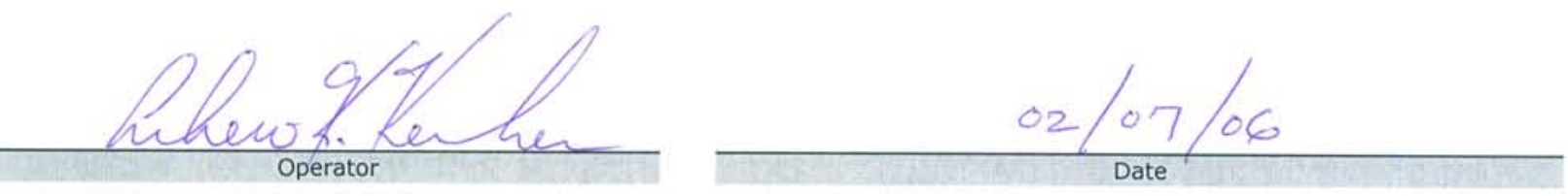
Data Report Form DRF-11A: Measurement of Buffer Layer Thickness

\begin{tabular}{|c|c|}
\hline Procedure: & AGR-CHAR-DAM-11 Rev. 1 \\
\hline Operator: & Andrew K. Kercher \\
\hline Folder name containing images: & IImc-agr|AGR\ImageProcessing|Completed_Layers\P06020701】 \\
\hline Sample ID: & LEU01-261-B01 \\
\hline Sample Description: & Variant 3b: IPyC/Buffer on BWXT kernel composite 69302 \\
\hline Folder name containing processed data: & |Imc-agr\AGR\ImageProcessing|Completed_Layers\P06020701_outpu \\
\hline
\end{tabular}

Number of buffer layers analyzed:

Mean of the average buffer thickness of each particle $(\mu \mathrm{m})$ : Standard deviation in the average buffer thickness of each particle $(\mu \mathrm{m})$ :

\section{5}

107.6

8.4

Distribution of the average buffer layer thickness (top binned)

\begin{tabular}{|c|c|}
\hline Buffer Thickness $(\mu \mathrm{m})$ & Frequency \\
\hline \hline 555 & 0 \\
\hline 60 & 0 \\
\hline 65 & 0 \\
\hline 70 & 0 \\
\hline 75 & 0 \\
\hline 80 & 0 \\
\hline 85 & 0 \\
\hline 90 & 1 \\
\hline 95 & 3 \\
\hline 100 & 11 \\
\hline 105 & 17 \\
\hline 110 & 15 \\
\hline 115 & 15 \\
\hline 120 & 8 \\
\hline 125 & 3 \\
\hline$>125$ & 2 \\
\hline & \\
\hline &
\end{tabular}

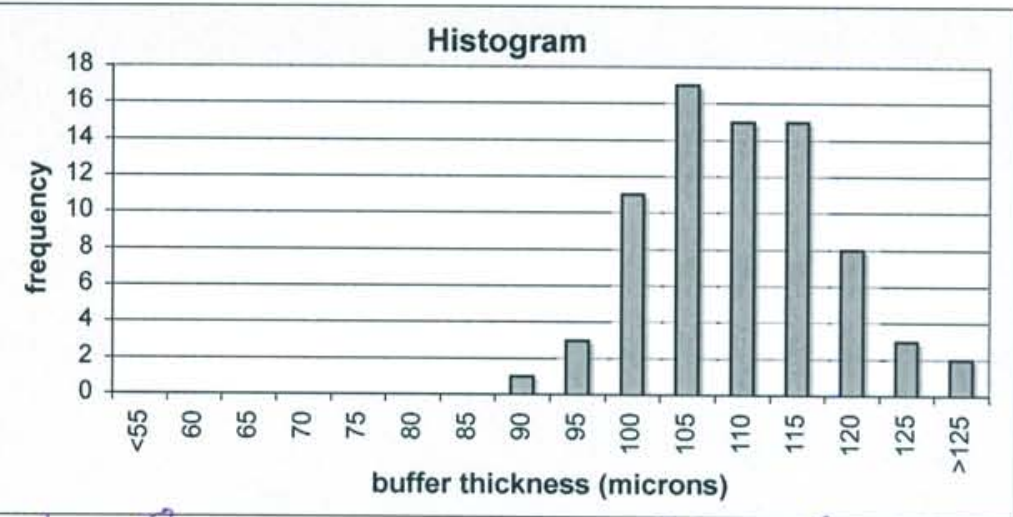

perator
02

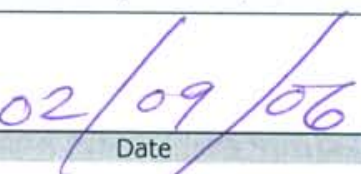


Data Report Form DRF-11B: Measurement of Inner Pyrocarbon Layer Thickness

\begin{tabular}{|c|c|}
\hline Procedure: & AGR-CHAR-DAM-11 Rev. 1 \\
\hline Operator: & Andrew K. Kercher \\
\hline Folder name containing images: & |IImc-agr|AGR|ImageProcessing|Completed_Layers\P06020701) \\
\hline Sample ID: & LEU01-26I-B01 \\
\hline Sample Description: & Variant 3b: IPyC/Buffer on BWXT kernel composite 69302 \\
\hline Folder name containing processed data: & IImc-agr|AGRIImageProcessing|Completed_Layers\P06020701_output| \\
\hline
\end{tabular}

Number of inner pyrocarbon layers analyzed: $\quad 80$

Mean of the average IPyC thickness of each particle $(\mu \mathrm{m}): \quad 35.1$

Standard deviation in the average IPyC thickness of each particle $(\mu \mathrm{m}):-13$

Distribution of the average IPyC layer thickness (top binned)

\begin{tabular}{|c|c|}
\hline IPyC Thickness $(\mu \mathrm{m})$ & Frequency \\
\hline \hline$<30$ & 2 \\
\hline 32 & 4 \\
\hline 34 & 22 \\
\hline 36 & 19 \\
\hline 38 & 24 \\
\hline 40 & 8 \\
\hline 42 & 1 \\
\hline 44 & 0 \\
\hline 46 & 0 \\
\hline 48 & 0 \\
\hline 50 & 0 \\
\hline 52 & 0 \\
\hline 54 & 0 \\
\hline 56 & 0 \\
\hline$>56$ & 0 \\
\hline & \\
\hline
\end{tabular}

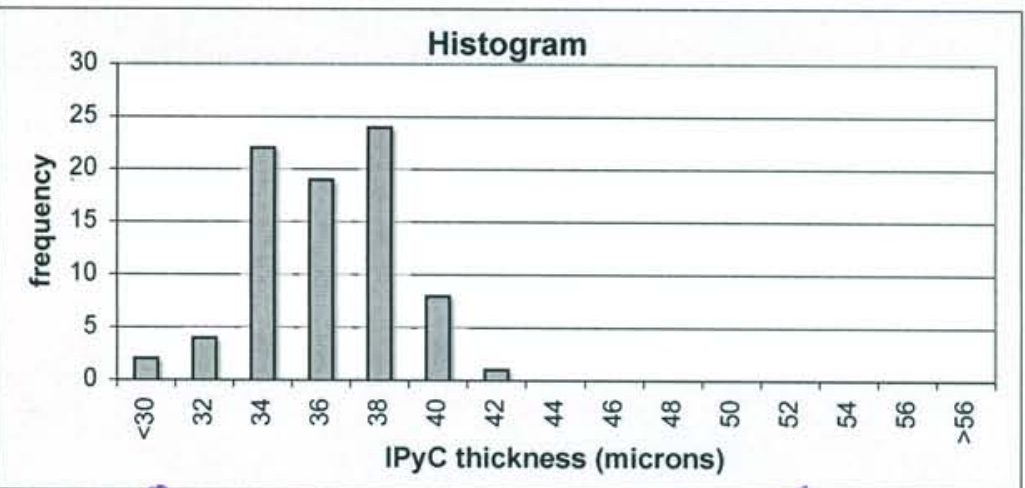


ORNL/TM-2006/019, Rev. 1

Data Report Form DRF-08: Imaging of Coated Particle Cross-sections Using an Optical Microscope System

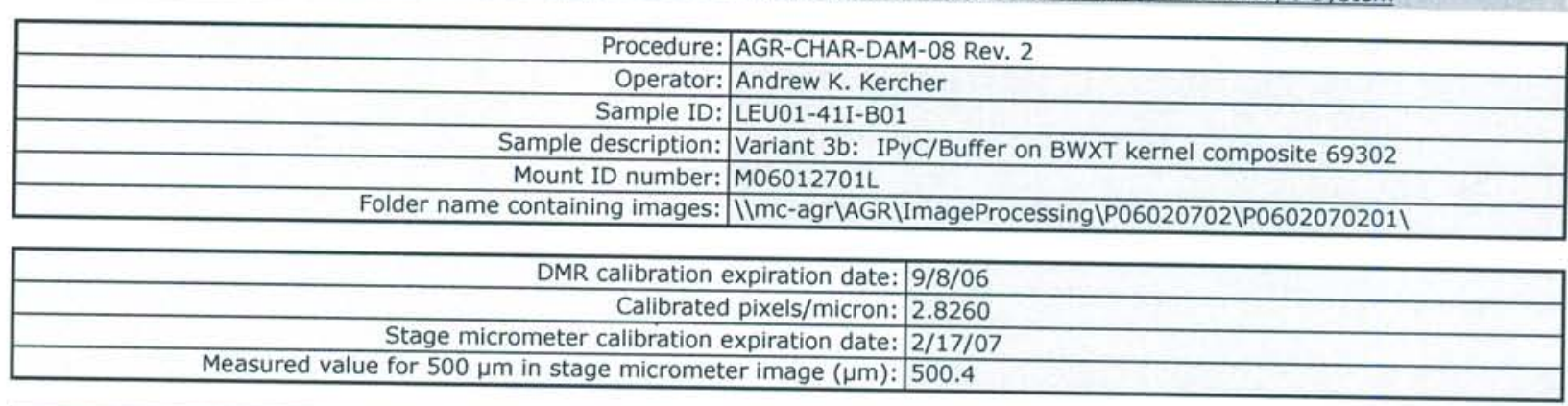

\begin{tabular}{|c|c|c|c|}
\hline \multicolumn{4}{|c|}{ Polish-down distance $\mathrm{n}, \mathrm{m}(\mu \mathrm{m})$} \\
\hline 2,2 & 2,8 & 8,2 & 8,8 \\
\hline \hline 308 & 297 & 314 & 300 \\
\hline
\end{tabular}

\begin{tabular}{|c|c|c|c|c|}
\hline \multicolumn{5}{|c|}{ Approximate layer width in polish plane $(\mu \mathrm{m})$} \\
\hline Kernel radius & Buffer & IPyC & SiC & OPyC \\
\hline \hline 168 & 111 & 36 & & \\
\hline
\end{tabular}
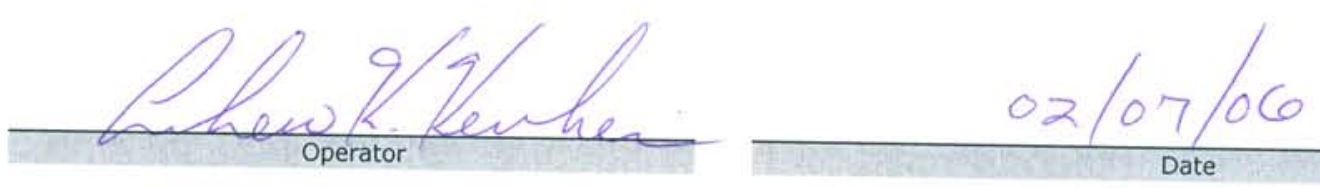
Data Report Form DRF-11A: Measurement of Buffer Layer Thickness

Procedure: AGR-CHAR-DAM-11 Rev. 1

Operator: Andrew K. Kercher

Folder name containing images: $\mid$ IImc-agr|AGR\ImageProcessing|Completed Layers\P06020702 Sample ID: LEU01-41I-B01

Sample Description: Variant 3b: IPyC/Buffer on BWXT kernel composite 69302

Folder name containing processed data: IImc-agr|AGR\ImageProcessing|Completed_Layers\P06020702_output\

Number of buffer layers analyzed: $\quad 72$

Mean of the average buffer thickness of each particle $(\mu \mathrm{m})$

Standard deviation in the average buffer thickness of each particle $(\mu \mathrm{m})$ :

110.1

Distribution of the average buffer layer thickness (top binned)

\begin{tabular}{|c|c|}
\hline Buffer Thickness $(\mu \mathrm{m})$ & Frequency \\
\hline \hline$<55$ & 0 \\
\hline 60 & 0 \\
\hline 65 & 0 \\
\hline 70 & 0 \\
\hline 75 & 0 \\
\hline 80 & 0 \\
\hline 85 & 0 \\
\hline 90 & 2 \\
\hline 95 & 4 \\
\hline 100 & 3 \\
\hline 105 & 8 \\
\hline 110 & 16 \\
\hline 115 & 19 \\
\hline 120 & 14 \\
\hline 125 & 3 \\
\hline$>125$ & 3 \\
\hline & \\
\hline
\end{tabular}
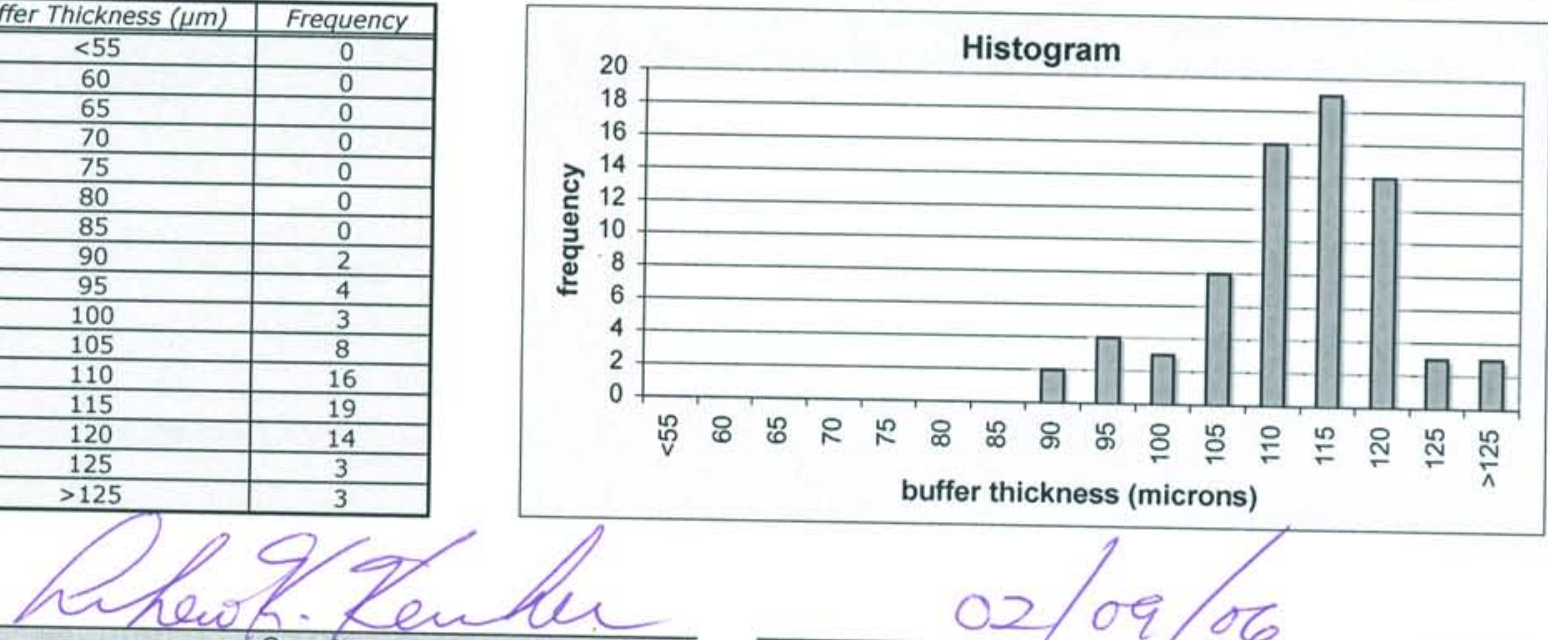

Operator

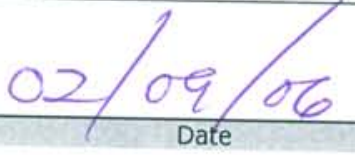


Data Report Form DRF-11B: Measurement of Inner Pyrocarbon Layer Thickness

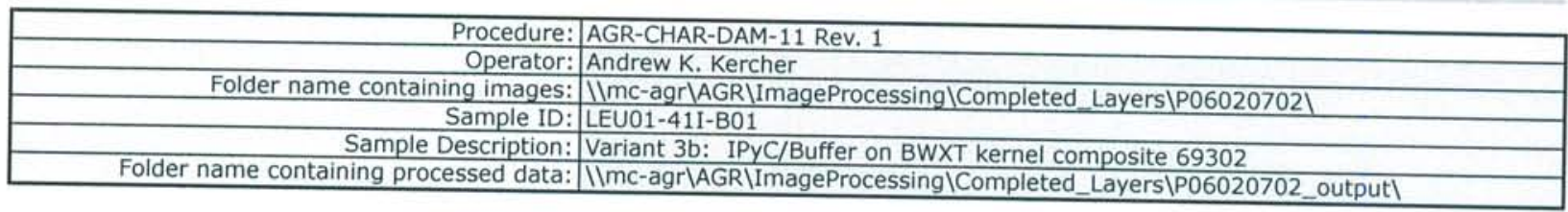

Number of inner pyrocarbon layers analyzed:

Mean of the average IPyC thickness of each particle $($ m $)$ :

Standard deviation in the average IPyC thickness of each particle $(\mu \mathrm{m})$ :

\section{Distribution of the average IPyC layer thickness (top binned)}

\begin{tabular}{|c|c|}
\hline IPyC Thickness $(\mu \mathrm{m})$ & Frequency \\
\hline \hline 30 & 0 \\
\hline 32 & 5 \\
\hline 34 & 20 \\
\hline 36 & 26 \\
\hline 38 & 24 \\
\hline 40 & 3 \\
\hline 42 & 1 \\
\hline 44 & 0 \\
\hline 46 & 0 \\
\hline 48 & 0 \\
\hline 50 & 0 \\
\hline 52 & 0 \\
\hline 54 & 0 \\
\hline 56 & 0 \\
\hline$>56$ & 0 \\
\hline
\end{tabular}
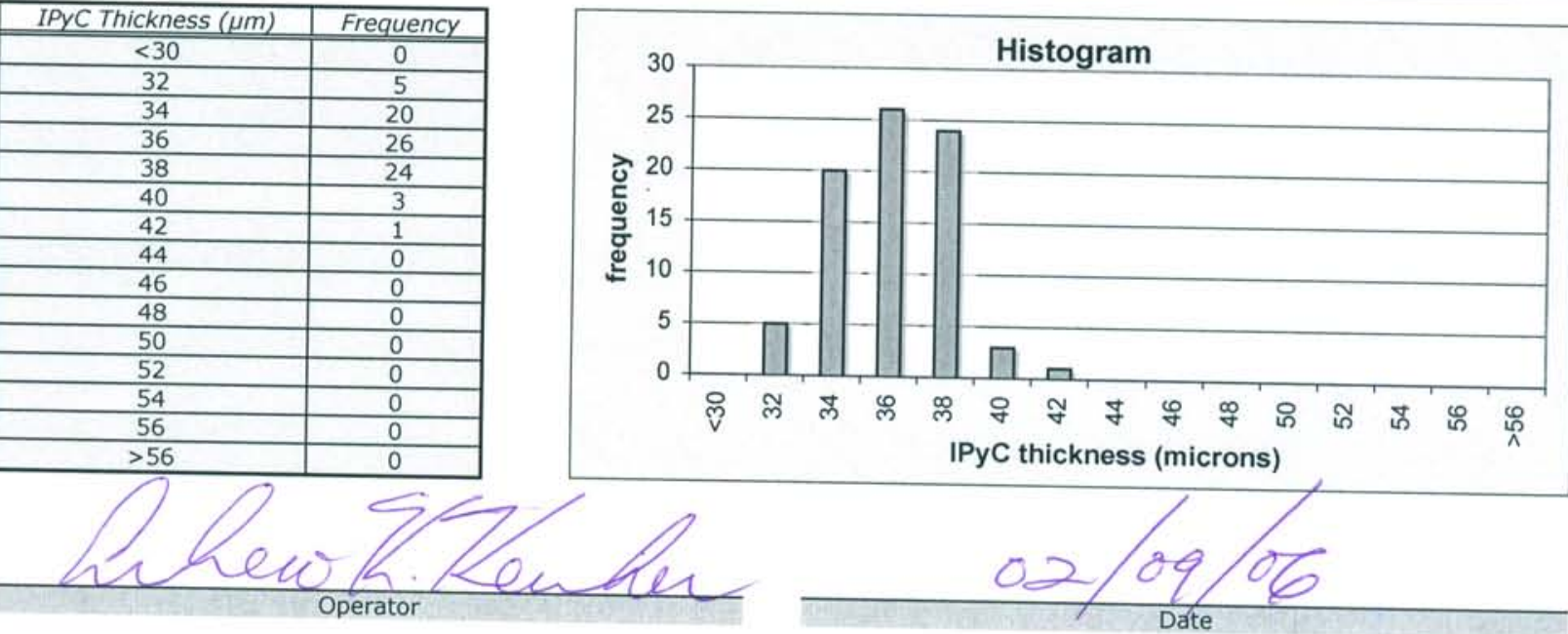


\section{Characterization of first batch of TRISO-coated particles}

This section contains data on LEU01-21T, the first batch of TRISO-coated particles used for the LEU01-46T particle composite. The data was obtained according to product inspection plan AGR-CHAR-PIP-03R2.

Note that some of the carbon deposited for the buffer layer reacted with the kernel to form a uranium carbide layer between the kernel and the buffer. The uranium carbide layer was not included in the measurement of the buffer thickness. The thickness of this carbide layer varied, but was typically around $6 \mu \mathrm{m}$ thick and effectively increased the kernel radius by that amount.

Note that the coating thicknesses of the TRISO-coated particle batch were remeasured after coated particle composite characterization was underway in order to improve the accuracy of the reported values. The original images of the particle cross sections were reanalyzed using an updated version of the image analysis program. This new version utilized a more robust algorithm for identifying the outer boundary of the outer pyrocarbon layer (OPyC). The data in this section shows the new coating thickness values. A record of the original measured values, which were initially used to determine the acceptance of this batch for inclusion in the composite, is included in section 16. Both the old and new coating thickness values for batch LEU01-21T were within the range specified in INL EDF-4380, Rev. 6.

The following pages show the inspection report form (IRF-03). Following IRF-03 are the individual data report forms for the measurements that were performed. Additional data at the end of this section is provided for information only. This batch was determined to satisfy the specifications in section 5.2 of EDF 4380, Rev. 6. 
ORNL/TM-2006/019, Rev. 1

Inspection Report Form IRF-03: Coated Particle Batches

Procedure: AGR-CHAR-PIP-03 Rev. 2

Coated particle batch ID: LEU 01-21T

Coated particle batch description: TRISO on BWXT kernel composite 69302

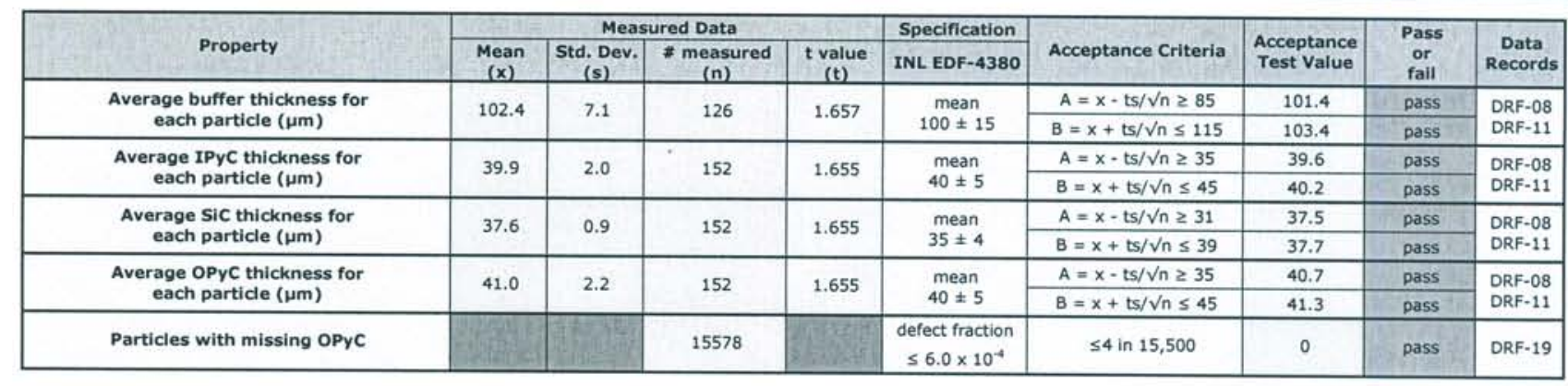

\begin{tabular}{|c|c|c|}
\hline Coldspot analysis performed on missing OPyC sample for information only. 10 out of 15578 gold spots observed.
\end{tabular}

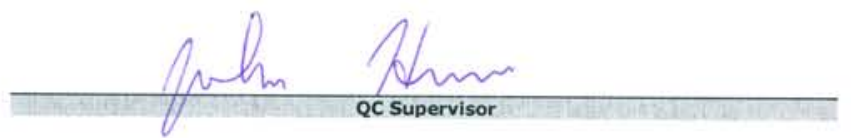

Accept Coated particle batch (Yes or No):

Yes

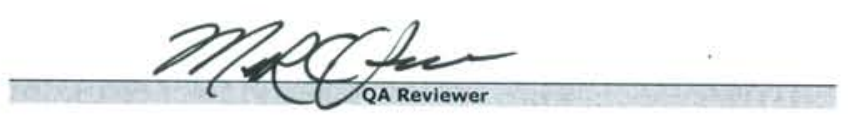

$3 / 29 / 06$

107 
Data Report Form DRF-08: Imaging of Coated Particle Cross-sections Using an Optical Microscope System

\begin{tabular}{|r|l|}
\hline Procedure: & AGR-CHAR-DAM-08 Rev. 0 \\
\hline Operator: & John Hunn \\
\hline Sample ID: & LEU01-21T-B01 \\
\hline Sample Description: & TRISO on BWXT kernel composite 69302 \\
\hline Mount Number(s): & M05112802 \\
\hline Folder name containing images: & \Imc-agr\AGR\ImageProcessing $\backslash$ P05112803 \\
\hline
\end{tabular}

DMR Calibration Expiration Date: $9 / 8 / 06$

Stage Micrometer Calibration Expiration Date: $2 / 17 / 07$

Measured Value for $500 \mu \mathrm{m}$ in Stage Micrometer Image: $500.4 \mu \mathrm{m}$
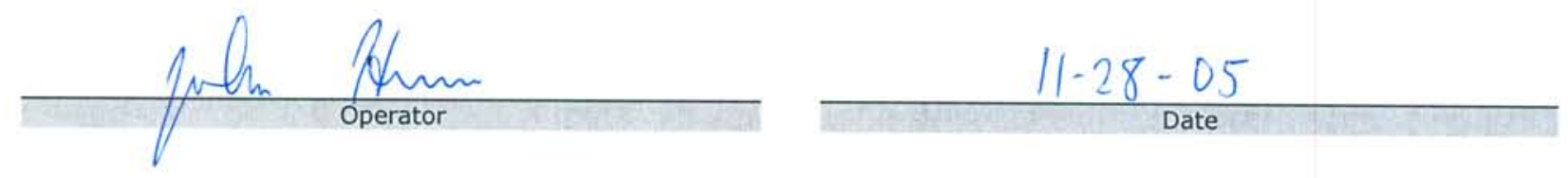
Data Report Form DRF-08: Imaging of Coated Particle Cross-sections Using an Optical Microscope System

\begin{tabular}{|r|l|}
\hline Procedure: & AGR-CHAR-DAM-08 Rev. 0 \\
\hline Operator: & John Hunn \\
\hline Sample ID: & LEU01-21T-B01 \\
\hline Sample Description: & TRISO on BWXT kernel composite 69302 \\
\hline Mount Number(s): & M05112803 \\
\hline Folder name containing images: & \Imc-agr\AGR\ImageProcessing \P05112804 \\
\hline
\end{tabular}

DMR Calibration Expiration Date: $9 / 8 / 06$

Stage Micrometer Calibration Expiration Date: $2 / 17 / 07$

Measured Value for $500 \mu \mathrm{m}$ in Stage Micrometer Image: $500.4 \mu \mathrm{m}$

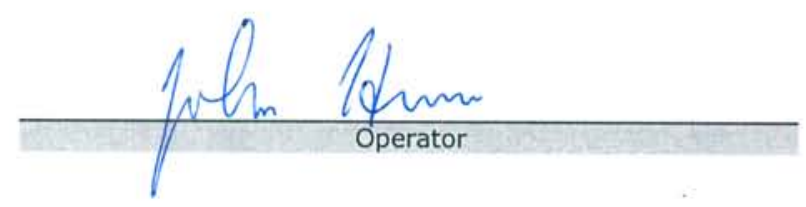

$$
11-28-05
$$




\section{Data Report Form DRF-11A: Measurement of Buffer Layer Thickness}

\begin{tabular}{|c|c|}
\hline Procedure: & AGR-CHAR-DAM-11 Rev. 1 \\
\hline Operator: & Andrew K. Kercher \\
\hline Folder name containing images: & |Imc-agr|AGR|ImageProcessing|Completed_Layers\P05112806। \\
\hline Sample ID: & LEU01-21T-B01 \\
\hline $\begin{array}{l}\text { Sample Description: } \\
\text { Folder name containing processed data: }\end{array}$ & \begin{tabular}{|l} 
Baseline TRISO on BWXT kernel composite 69302 \\
IImc-agr|AGRIImageProcessing|Completed Layers\P05112806 out
\end{tabular} \\
\hline
\end{tabular}

Number of buffer layers analyzed: $\quad 126$

Mean of the average buffer thickness of each particle $(\mu \mathrm{m}): \quad 102.4$

Standard deviation in the average buffer thickness of each particle $(\mu \mathrm{m})$ :

Distribution of the average buffer layer thickness (top binned)

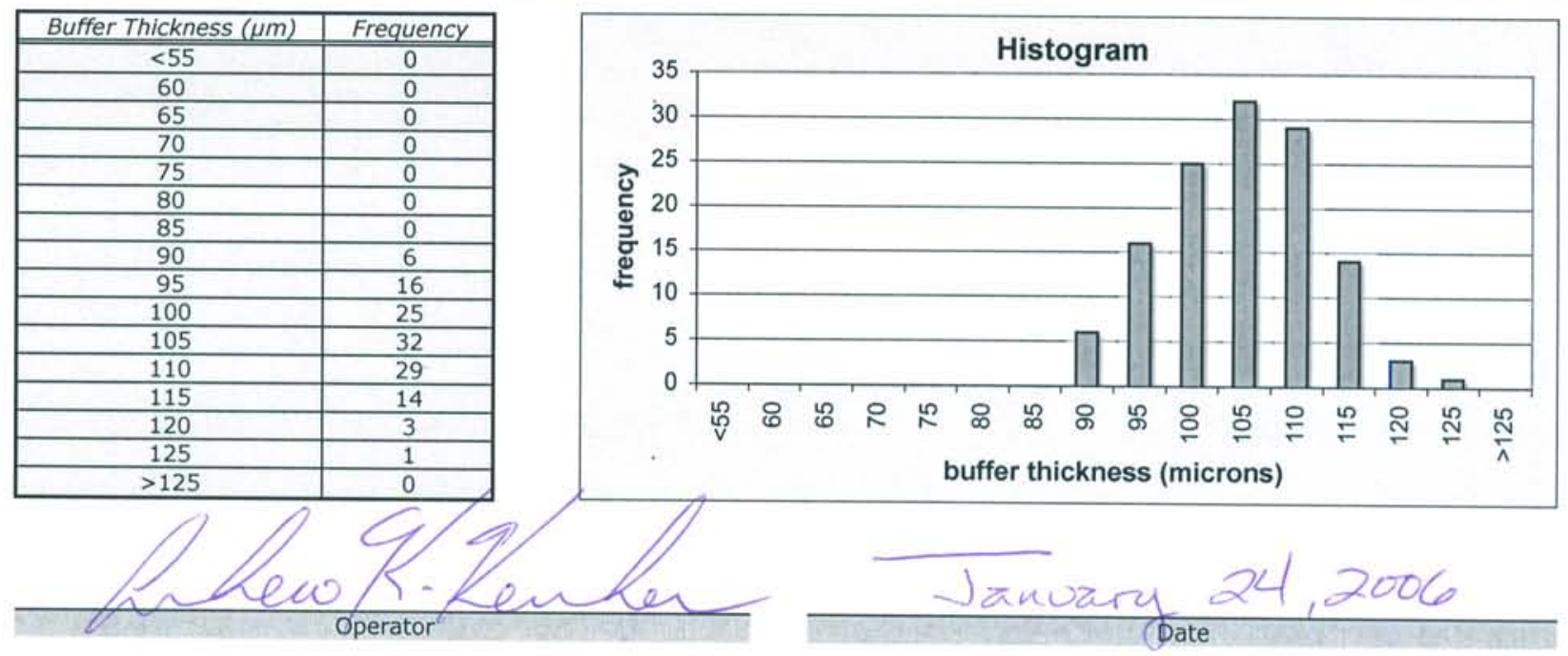


Data Report Form DRF-11B: Measurement of Inner Pyrocarbon Layer Thickness

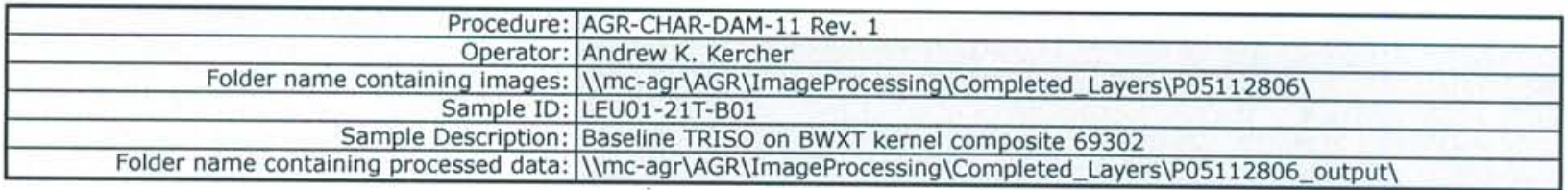

Number of inner pyrocarbon layers analyzed:

Mean of the average IPyC thickness of each particle $(\mu \mathrm{m})$ :

Standard deviation in the average IPyC thickness of each particle $(\mu \mathrm{m})$ :

Distribution of the average IPyC layer thickness (top binned)

\begin{tabular}{|c|c|}
\hline IPyC Thickness $(\mu \mathrm{m})$ & Frequency \\
\hline \hline$<30$ & 0 \\
\hline 32 & 0 \\
\hline 34 & 0 \\
\hline 36 & 2 \\
\hline 38 & 20 \\
\hline 40 & 63 \\
\hline 42 & 42 \\
\hline 44 & 19 \\
\hline 46 & 6 \\
\hline 48 & 0 \\
\hline 50 & 0 \\
\hline 52 & 0 \\
\hline 54 & 0 \\
\hline 56 & 0 \\
\hline$>56$ & 0 \\
\hline & \\
\hline
\end{tabular}
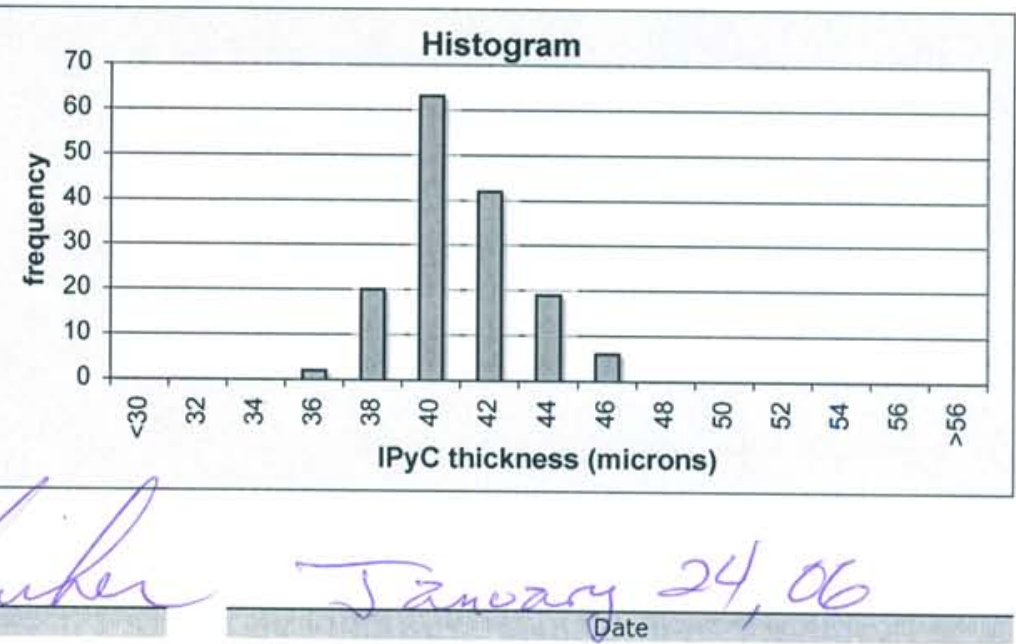
Data Report Form DRF-11C: Measurement of Silicon Carbide Layer Thickness

\begin{tabular}{|c|c|}
\hline Procedure: & AGR-CHAR-DAM-11 Rev. 1 \\
\hline Operator: & Andrew K. Kercher \\
\hline Folder name containing images: & IImc-agr|AGR\ImageProcessing|Completed_Layers\P05112806| \\
\hline Sample ID: & LEU01-21T-B01 \\
\hline Sample Description: & Baseline TRISO on BWXT kernel composite 69302 \\
\hline Folder name containing processed data: & 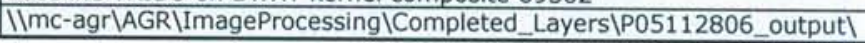 \\
\hline
\end{tabular}

Number of silicon carbide layers analyzed: $\quad 152$

Mean of the average SiC thickness of each particle $(\mu \mathrm{m}):-37.6$

Standard deviation in the average SiC thickness of each particle $(\mu \mathrm{m})$ :

0.9

Distribution of the average SiC layer thickness (top binned)

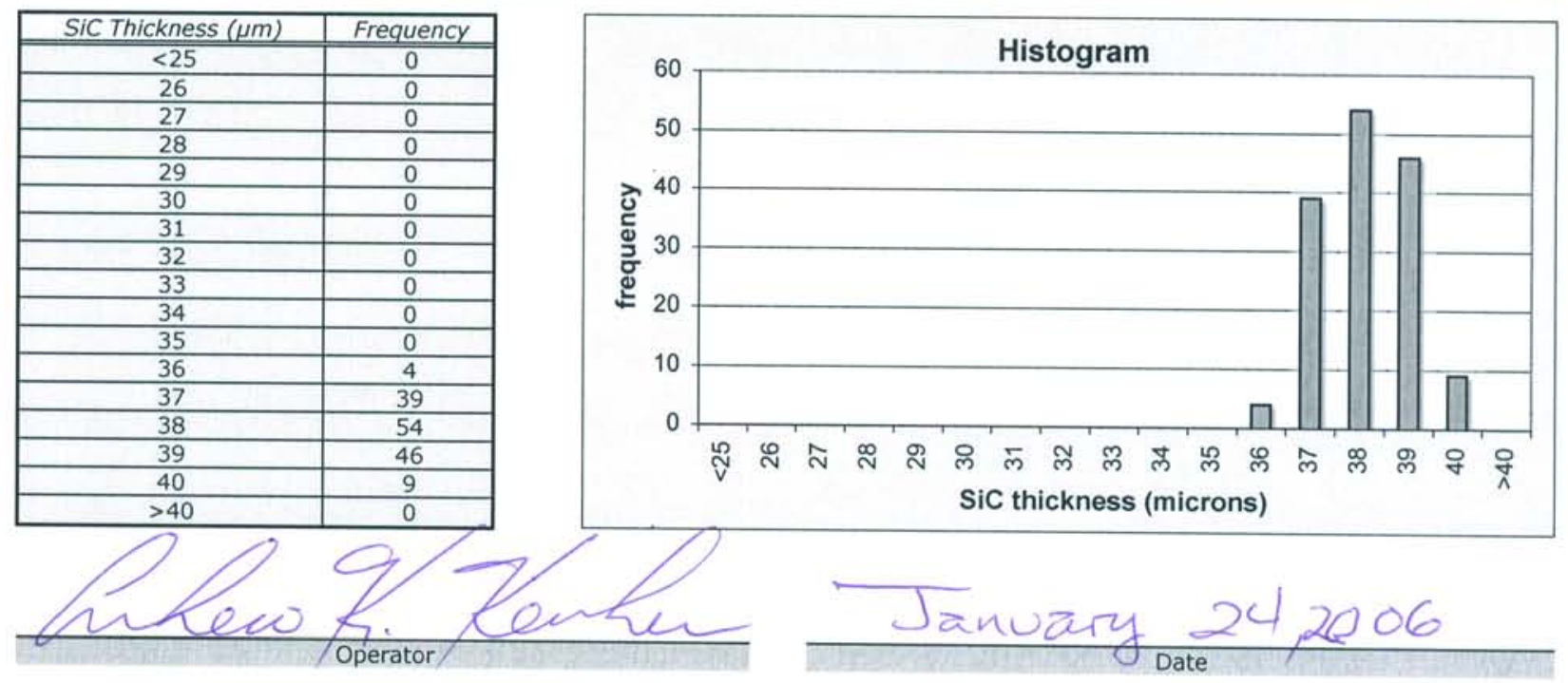


Data Report Form DRF-11D: Measurement of Outer Pyrocarbon Layer Thickness

Procedure: AGR-CHAR-DAM-11 Rev. 1

Operator: Andrew K. Kercher

Folder Name: |Imc-agr|AGR|ImageProcessing|Completed_Layers\P05112806|

Sample ID: LEU01-21T-B01

Sample Description: Baseline TRISO on BWXT kernel composite 69302

Folder name containing processed data: IImc-agr|AGR\ImageProcessing|Completed_Layers\P05112806_output|

Number of outer pyrocarbon layers analyzed: $\quad 152$

Mean of the average OPyC thickness of each particle $(\mu \mathrm{m}):$

Standard deviation in the average OPyC thickness of each particle $(\mu \mathrm{m})$ :

2.2

Distribution of the average OPYC layer thickness (top binned)

\begin{tabular}{|c|c|}
\hline OPyC Thickness $(\mu \mathrm{m})$ & Frequency \\
\hline \hline$<20$ & 0 \\
\hline 22 & 0 \\
\hline 24 & 0 \\
\hline 26 & 0 \\
\hline 28 & 0 \\
\hline 30 & 1 \\
\hline 32 & 0 \\
\hline 34 & 0 \\
\hline 36 & 0 \\
\hline 38 & 6 \\
\hline 40 & 42 \\
\hline 42 & 58 \\
\hline 44 & 34 \\
\hline 46 & 9 \\
\hline 48 & 2 \\
\hline 50 & 0 \\
\hline$>50$ & 0 \\
\hline
\end{tabular}
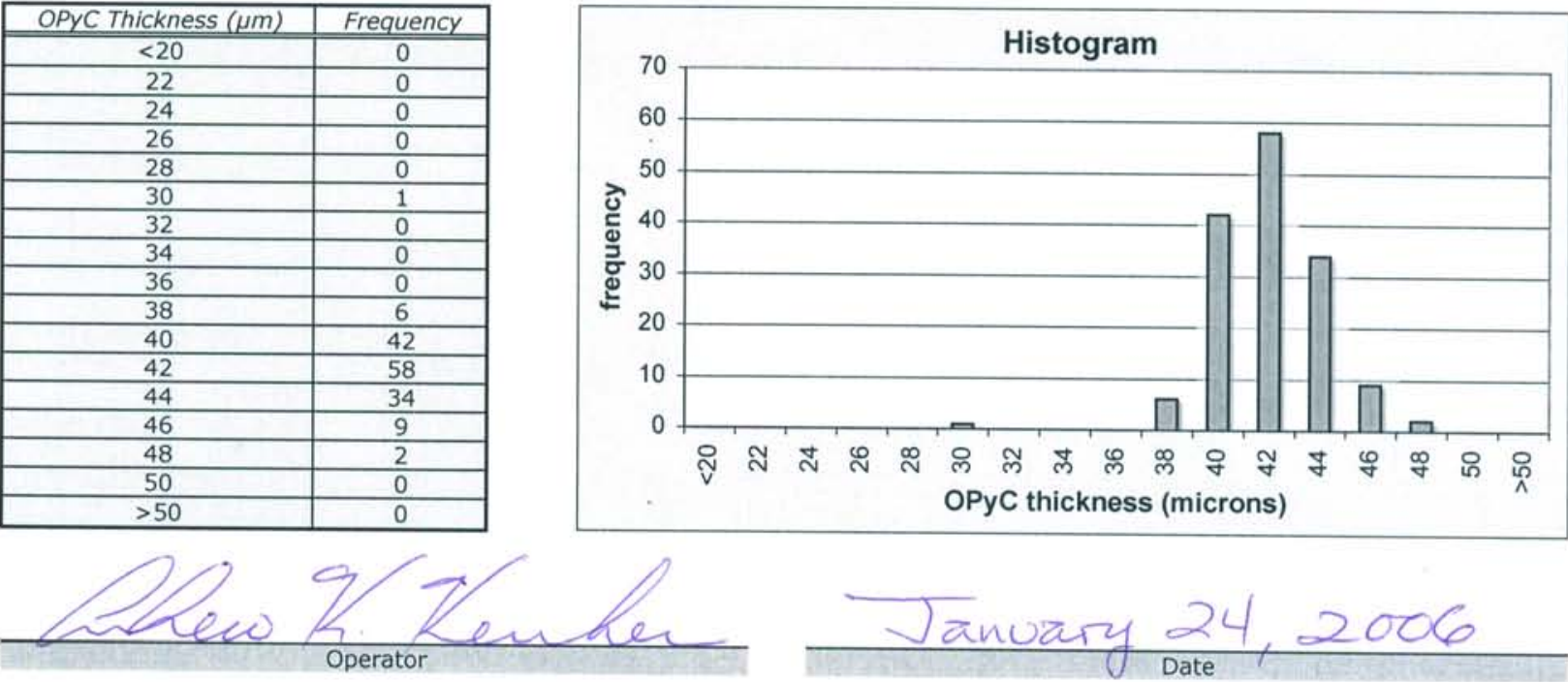

January 24,2006 
Data Report Form DRF-19: Counting of Particles with Missing OPyC Layer by Visual Inspection

\begin{tabular}{|r|l|}
\hline Procedure: & AGR-CHAR-DAM-19 Rev. 1 \\
\hline Operator: & John Hunn \\
\hline Sample ID: & LEU01-21T-C02 \\
\hline Sample Description: & TRISO on BWXT kernel composite 69302 \\
\hline Filename: & IImc-agr\AGR\MissingOPyCIX05112801_DRF19R1.xIs \\
\hline
\end{tabular}

Mean average weight/particle $(\mathrm{g}): 7.30 \mathrm{E}-04$ Uncertainty in average weight/particle $(g): 1.48 \mathrm{E}-06$

Weight of sample of particles $(\mathrm{g}): 11.372$

Approximate number of particles in sample: 15578

Uncertainty in number of particles in sample: 32

Number of particles with missing OPyC layer: 0

Comments on unusual visual characteristics of OPyC

A few particles $(<10)$ were observed to have a dark blemish as seen in image P0511280501.

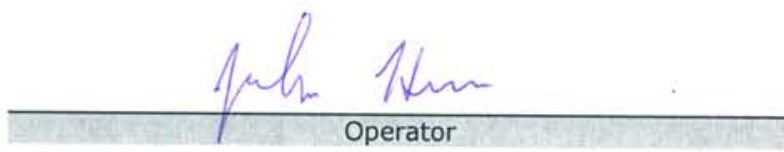

\section{$11-28.05$}




\section{For Information Only}

The information in the remainder of this section reports results of measurements not required by the fuel specification and is provided for information only. 
Data Report Form DRF-22: Estimation of Average Particle Weight

Procedure: AGR-CHAR-DAM-22 Rev. 1

Operator: Dixie Barker

Particle Lot ID: LEU01-21T-D01

Particle Lot Description: TRISO on LEU01 kernels

Filename: \Imc-agr\AGR\ParticleWeight|W05112301_DRF22R1.xls

\begin{tabular}{|r|c|c|c|c|c|}
\hline & Sample 1 & Sample 2 & Sample 3 & Sample 4 & Sample 5 \\
\hline Weight of particles (g): & $9.46 \mathrm{E}-02$ & $8.24 \mathrm{E}-02$ & $8.75 \mathrm{E}-02$ & $8.89 \mathrm{E}-02$ & $8.36 \mathrm{E}-02$ \\
\hline Number of particles: & 130 & 113 & 119 & 122 & 115 \\
\hline Average weight/particle $(\mathrm{g}):$ & $7.28 \mathrm{E}-04$ & $7.29 \mathrm{E}-04$ & $7.35 \mathrm{E}-04$ & $7.29 \mathrm{E}-04$ & $7.27 \mathrm{E}-04$ \\
\hline
\end{tabular}

Mean average weight/particle $(g): 7.30 \mathrm{E}-04$

Uncertainty in mean average weight/particle $(\mathrm{g}): 1.48 \mathrm{E}-06$

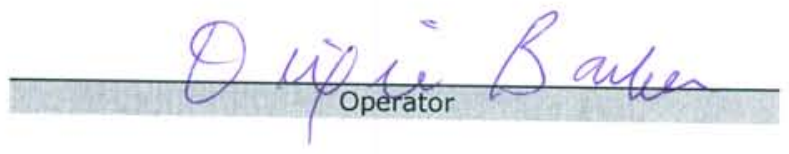

$$
\frac{11-23-0,5}{\text { Date }}
$$


Data Report Form DRF-20: Counting of Particles with SiC Gold Spot Defects by Visual Inspection

Procedure: AGR-CHAR-DAM-20 Rev. 1

Operator: John Hunn

Sample ID: LEU01-21T-C02

Sample Description: TRISO on BWXT kernel composite 69302

Filename: \Imc-agr|AGR\GoldSpots\G05112901_DRF20R1.xls

Mean average weight/particle $(\mathrm{g}): 7.30 \mathrm{E}-04$

Uncertainty in average weight/particle $(g): 1.48 \mathrm{E}-06$

Weight of sample of particles $(\mathrm{g}): 11.372$

Approximate number of particles in sample: 15578

Uncertainty in number of particles in sample: 32

Number of particles with gold spot defects: 10

\section{Comments on unusual visual characteristics of SiC}

Goldspots ranged from yellow to golden-brown.

Mounted for cross section in M05112901.

Images of cross sections showed soot inclusion at IPyC/SiC interface.

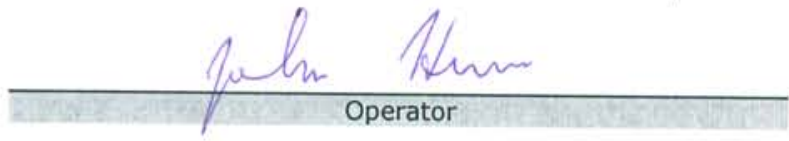

$$
11-29-05
$$


$10 / 15578$ gold spots found.

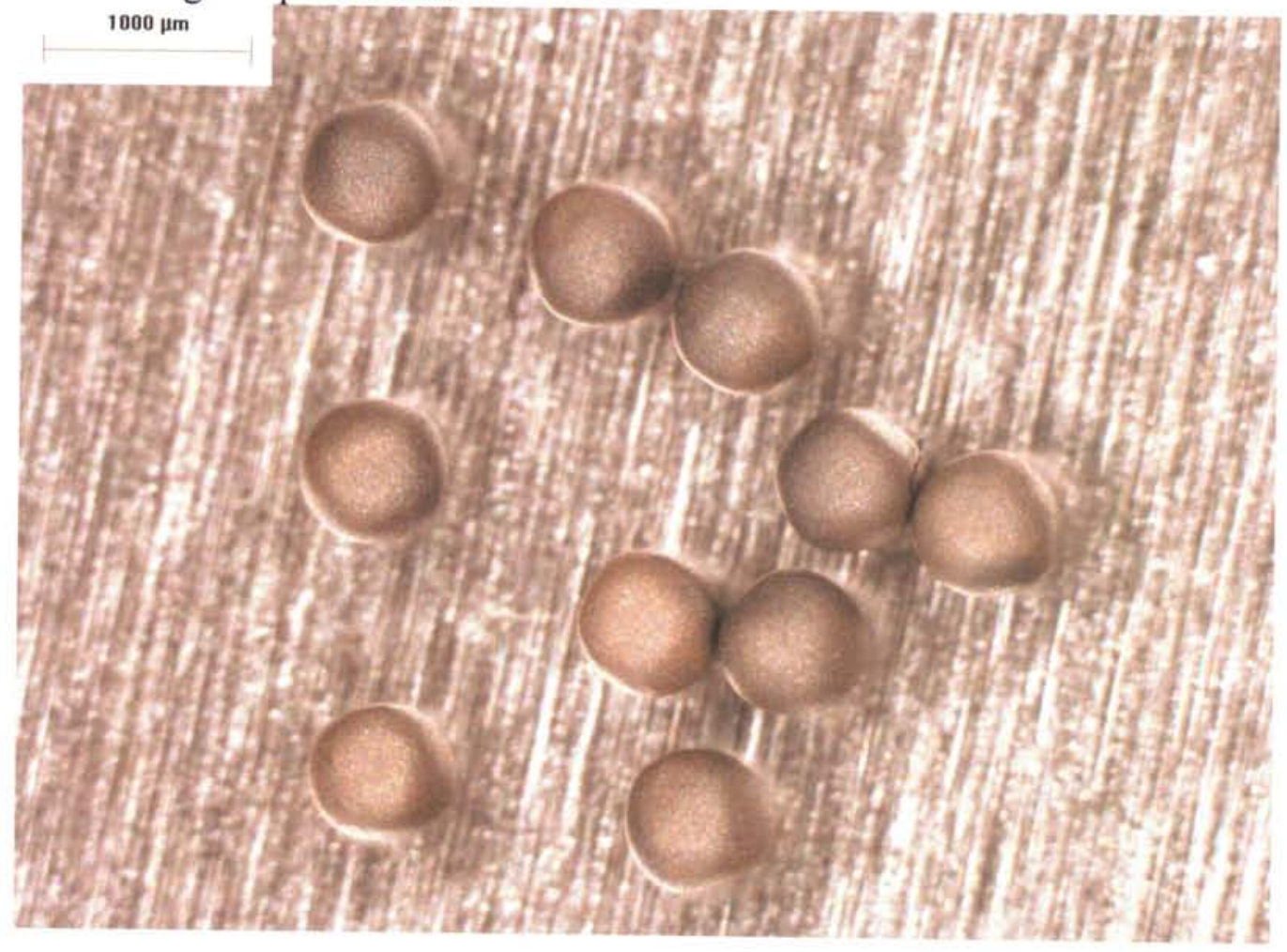

Example of soot inclusion at IPyC/SiC interface on particle with a goldspot. $200 \mu \mathrm{m}$

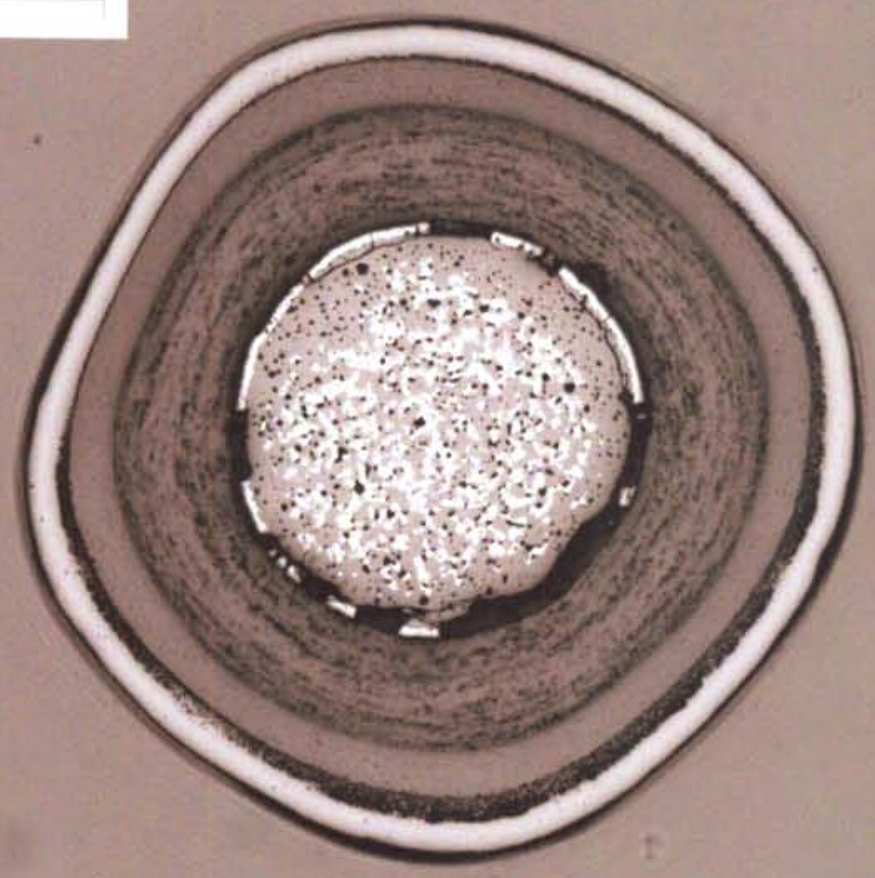


Data Report Form DRF-20: Counting of Particles with SiC Gold Spot Defects by Visual Inspection

Procedure: AGR-CHAR-DAM-20 Rev. 1

Operator: John Hunn

Sample ID: LEU01-21T-A01

Sample Description: TRISO on BWXT kernel composite 69302

Filename: $\mid \backslash m c$-agr\AGR\GoldSpots\G05120101_DRF20R1.xIs

Mean average weight/particle $(\mathrm{g}): 7.30 \mathrm{E}-04$

Uncertainty in average weight/particle $(\mathrm{g}): 1.48 \mathrm{E}-06$

Weight of sample of particles $(\mathrm{g}): 5.286$

Approximate number of particles in sample: 7241

Uncertainty in number of particles in sample: 15

Number of particles with gold spot defects: 5

Comments on unusual visual characteristics of SiC

Additional sample analyzed to go with G05112901_DRF20R1.xls, which was inconclusive as to whether LEU01-21 would pass the composite goldspot specification.

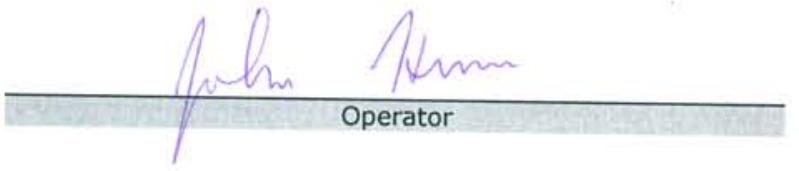

$\frac{|2-0|-05}{\text { Date }}$


Data Report Form DRF-20: Counting of Particles with SiC Gold Spot Defects by Visual Inspection

\begin{tabular}{|r|l|}
\hline Procedure: & AGR-CHAR-DAM-20 Rev. 1 \\
\hline Operator: & John Hunn \\
\hline Sample ID: & LEU01-21T-A01 and LEU01-21T-C02 \\
\hline Sample Description: & TRISO on BWXT kernel composite 69302 \\
\hline Filename: & IIMc-agr|AGR\GoldSpots\G05120102_DRF20R1.xIs \\
\hline
\end{tabular}

Mean average weight/particle $(g): 07.30 \mathrm{E}-04$

Uncertainty in average weight/particle $(\mathrm{g}): 1.48 \mathrm{E}-06$

Weight of sample of particles $(\mathrm{g}): 16.658$

Approximate number of particles in sample: 22819

Uncertainty in number of particles in sample: 46

Number of particles with gold spot defects: 15

Comments on unusual visual characteristics of $\mathrm{SiC}$

This batch would not pass the composite particle specification without further analysis.

Mnm Prom

$\frac{12-01-05}{\text { Date }}$ 


\section{Characterization of second batch of TRISO-coated particles}

This section contains data on LEU01-35T, the second batch of TRISO-coated particles used for the LEU01-46T particle composite. The data was obtained according to product inspection plan AGR-CHAR-PIP-03R2.

Note that some of the carbon deposited for the buffer layer reacted with the kernel to form a uranium carbide layer between the kernel and the buffer. The uranium carbide layer was not included in the measurement of the buffer thickness. The thickness of this carbide layer varied, but was typically around $6 \mu \mathrm{m}$ thick and effectively increased the kernel radius by that amount.

Note that the coating thicknesses of the TRISO-coated particle batch were remeasured after coated particle composite characterization was underway in order to improve the accuracy of the reported values. The original images of the particle cross sections were reanalyzed using an updated version of the image analysis program. This new version utilized a more robust algorithm for identifying the outer boundary of the outer pyrocarbon layer (OPyC). The data in this section shows the new coating thickness values. A record of the original measured values, which were initially used to determine the acceptance of this batch for inclusion in the composite, is included in section 16. Both the old and new coating thickness values for batch LEU01-35T were within the range specified in INL EDF-4380, Rev. 6.

The following pages show the inspection report form (IRF-03). Following IRF-03 are the individual data report forms for the measurements that were performed. Additional data at the end of this section is provided for information only. This batch was determined to satisfy the specifications in section 5.2 of EDF 4380, Rev. 6. 
ORNL/TM-2006/019, Rev. 1

Inspection Report Form IRF-03: Coated Particle Batches

Procedure: AGR-CHAR-PIP-03 Rev, 2

Coated particle batch ID: LEU01-35T

Coated particle batch description: TRISO on BWXT kernel composite 69302

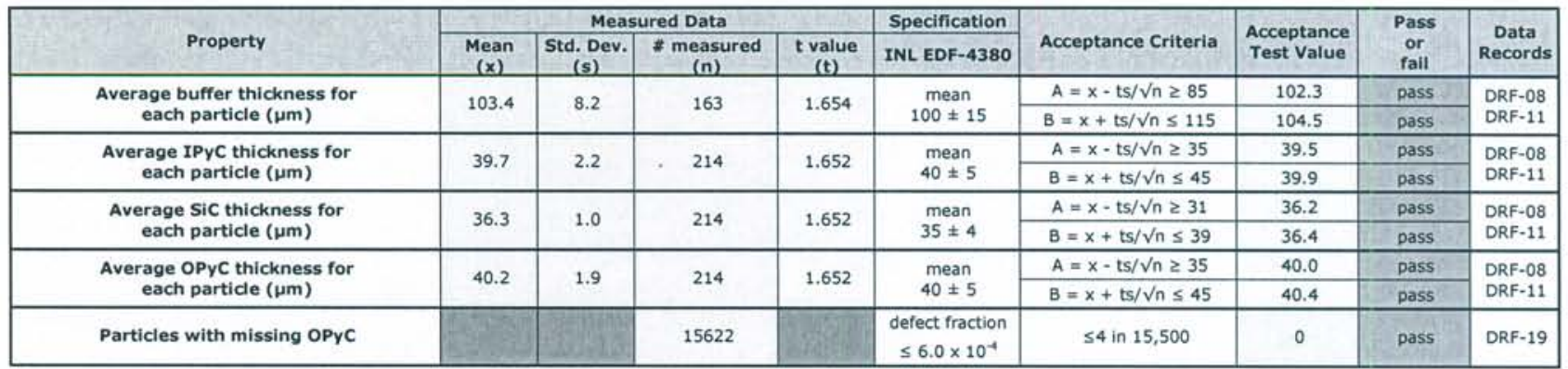

Comments

Goldspot analysis performed on missing OPyC sample for information only. 17 out of 15622 gold spots observed.

\section{Mln $/ 4 n$ \\ QC Supervisor}

Yes

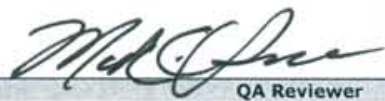

Accept Coated particle batch (Yes or No)

QA Reviewer
$3-23-06$

Date

$3-23-06$

$3 / 2 \% / 06$ 
ORNL/TM-2006/019, Rev. 1

Data Report Form DRF-08: Imaging of Coated Particle Cross-sections Using an Optical Microscope System

\begin{tabular}{|r|l|}
\hline Procedure: & AGR-CHAR-DAM-08 Rev. 2 \\
\hline Operator: & Andrew K. Kercher \\
\hline Sample ID: & LEU01-35T-B01 \\
\hline Sample description: & TRISO on BWXT kernel composite 69302 \\
\hline Mount ID number: & M05121201L \\
\hline Folder name containing images: & IImc-agr\AGR \ImageProcessing\Pyymmddnn\P05121202\} $\\
{\hline}$
\end{tabular}

\begin{tabular}{|r|r|}
\hline DMR calibration expiration date: & $9 / 8 / 06$ \\
\hline Calibrated pixels/micron: & 2.8260 \\
\hline Stage micrometer calibration expiration date: & $2 / 17 / 07$ \\
\hline Measured value for $500 \mu \mathrm{m}$ in stage micrometer image $(\mu \mathrm{m}):$ & 500.4 \\
\hline
\end{tabular}

\begin{tabular}{|c|c|c|c|}
\hline \multicolumn{4}{|c|}{ Polish-down distance $n, m(\mu \mathrm{m})$} \\
\hline 2,2 & 2,8 & 8,2 & 8,8 \\
\hline \hline 367 & 371 & 380 & 390 \\
\hline
\end{tabular}

\begin{tabular}{|c|c|c|c|c|}
\hline \multicolumn{5}{|c|}{ Approximate layer width in polish plane $(\mu \mathrm{m})$} \\
\hline Kernel radius & Buffer & IPyC & SiC & OPyC \\
\hline \hline 177 & 108 & 38 & 38 & 39 \\
\hline
\end{tabular}

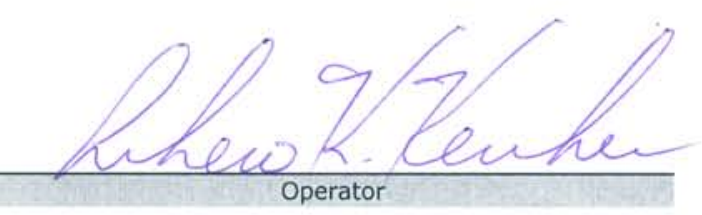

$$
12 / 12 / 05
$$


ORNL/TM-2006/019, Rev. 1

Data Report Form DRF-08: Imaging of Coated Particle Cross-sections Using an Optical Microscope System

\begin{tabular}{|r|l|}
\hline Procedure: & AGR-CHAR-DAM-08 Rev. 2 \\
\hline Operator: & Andrew K. Kercher \\
\hline Sample ID: & LEU01-35T-B01 \\
\hline Sample description: & TRISO on BWXT kernel composite 69302 \\
\hline Mount ID number: & M05121202L \\
\hline Folder name containing images: & IIMC-agr\AGR\ImageProcessing\Pyymmddnn\P05121202 \\
\hline
\end{tabular}

\begin{tabular}{|r|r|}
\hline DMR calibration expiration date: & $9 / 8 / 06$ \\
\hline Calibrated pixels/micron: & 2.8260 \\
\hline Stage micrometer calibration expiration date: & $2 / 17 / 07$ \\
\hline Measured value for $500 \mu \mathrm{m}$ in stage micrometer image $(\mu \mathrm{m}):$ & 500.4 \\
\hline
\end{tabular}

\begin{tabular}{|c|c|c|c|}
\hline \multicolumn{4}{|c|}{ Polish-down distance $n, m(\mu \mathrm{m})$} \\
\hline 2,2 & 2,8 & 8,2 & 8,8 \\
\hline \hline 383 & 394 & 384 & 399 \\
\hline
\end{tabular}

\begin{tabular}{|c|c|c|c|c|}
\hline \multicolumn{5}{|c|}{ Approximate layer width in polish plane $(\mu \mathrm{m})$} \\
\hline Kernel radius & Buffer & IPyC & SiC & OPyC \\
\hline \hline 170 & 94 & 40 & 38 & 40 \\
\hline
\end{tabular}
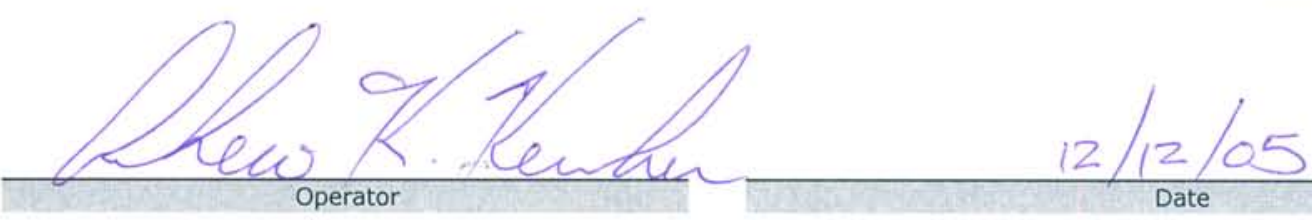
ORNL/TM-2006/019, Rev. 1

Data Report Form DRF-08: Imaging of Coated Particle Cross-sections Using an Optical Microscope System

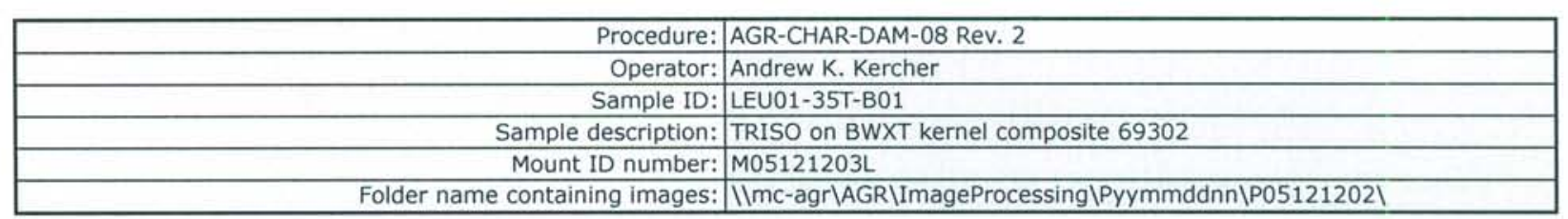

\begin{tabular}{|r|l|}
\hline DMR calibration expiration date: & $9 / 8 / 06$ \\
\hline Calibrated pixels/micron: & 2.8260 \\
\hline Stage micrometer calibration expiration date: & $2 / 17 / 07$ \\
\hline Measured value for $500 \mu \mathrm{m}$ in stage micrometer image $(\mu \mathrm{m}):$ & 500.4 \\
\hline
\end{tabular}

\begin{tabular}{|c|c|c|c|}
\hline \multicolumn{4}{|c|}{ Polish-down distance $n, m(\mu \mathrm{m})$} \\
\hline 2,2 & 2,8 & 8,2 & 8,8 \\
\hline \hline 356 & 369 & 346 & 360 \\
\hline
\end{tabular}

\begin{tabular}{|c|c|c|c|c|}
\hline \multicolumn{5}{|c|}{ Approximate layer width in polish plane $(\mu \mathrm{m})$} \\
\hline Kernel radius & Buffer & IPyC & SiC & OPyC \\
\hline \hline 169 & 107 & 38 & 39 & 40 \\
\hline
\end{tabular}
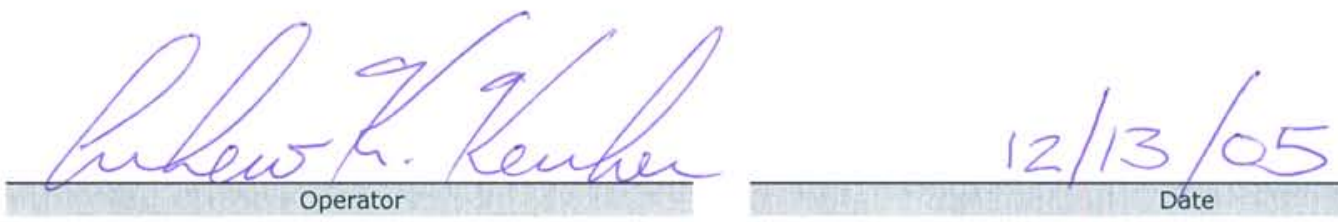
Data Report Form DRF-11A: Measurement of Buffer Layer Thickness

\begin{tabular}{|c|c|}
\hline Procedure: & AGR-CHAR-DAM-11 Rev. 1 \\
\hline Operator: & Andrew K. Kercher \\
\hline Folder name containing images: & 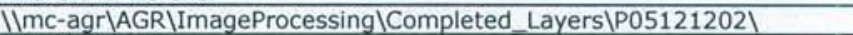 \\
\hline Sample ID: & LEU01-35T-B01 \\
\hline Sample Description: & Baseline TRISO on BWXT kernel composite 69302 \\
\hline Folder name containing processed data: & |Imc-agr\AGR\ImageProcessing|Completed_Layers\P05121202_output। \\
\hline
\end{tabular}

\begin{tabular}{r|r|}
\hline Number of buffer layers analyzed: & 163 \\
\hline Mean of the average buffer thickness of each particle $(\mu \mathrm{m}):$ & 103.4 \\
\hline Standard deviation in the average buffer thickness of each particle $(\mu \mathrm{m}):$ & 8.2 \\
\hline
\end{tabular}

\section{Distribution of the average buffer layer thickness (top binned)}

\begin{tabular}{|c|c|}
\hline Buffer Thickness $(\mu \mathrm{m})$ & Frequency \\
\hline \hline$<55$ & 0 \\
\hline 60 & 0 \\
\hline 65 & 0 \\
\hline 70 & 0 \\
\hline 75 & 0 \\
\hline 80 & 0 \\
\hline 85 & 1 \\
\hline 90 & 3 \\
\hline 95 & 20 \\
\hline 100 & 37 \\
\hline 105 & 39 \\
\hline 110 & 29 \\
\hline 115 & 19 \\
\hline 120 & 10 \\
\hline 125 & 3 \\
\hline$>125$ & 2 \\
\hline
\end{tabular}
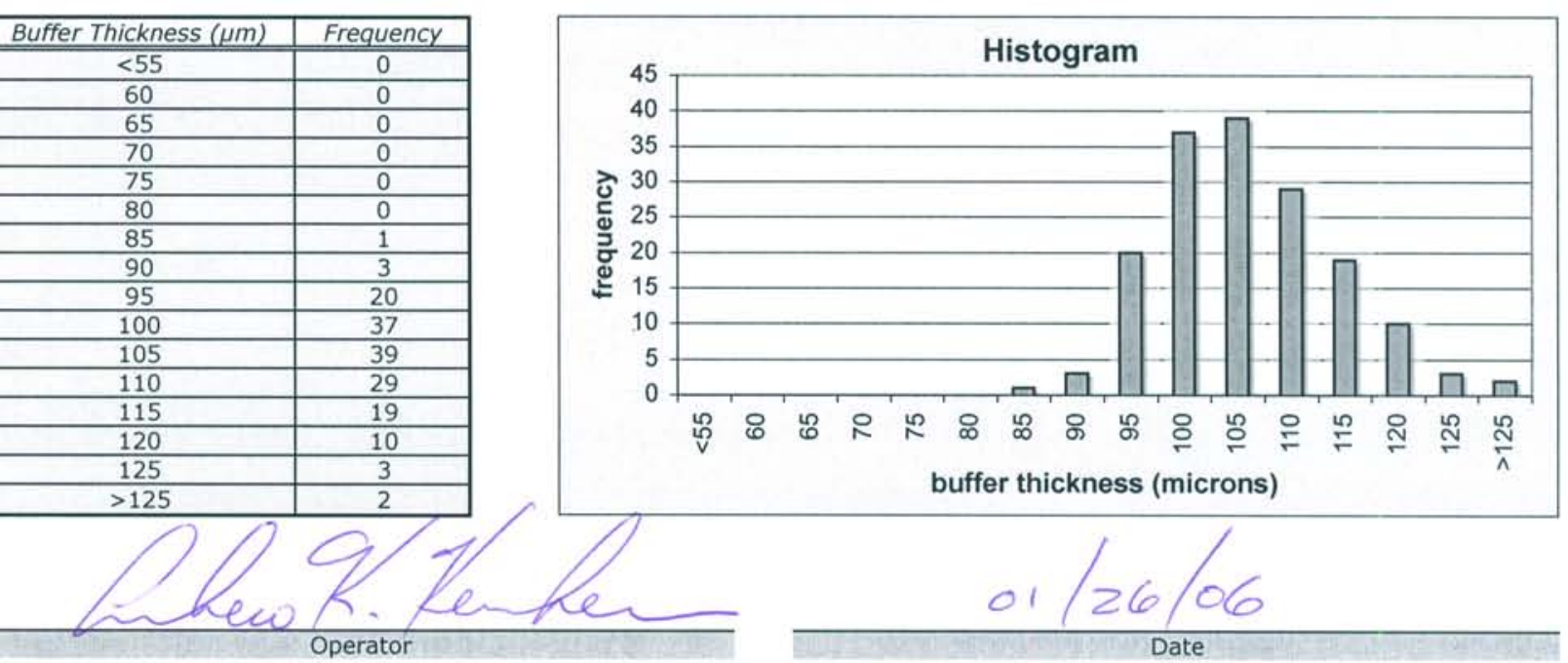


\section{Data Report Form DRF-11B: Measurement of Inner Pyrocarbon Layer Thickness}

\begin{tabular}{|c|c|}
\hline Procedure: & AGR-CHAR-DAM-11 Rev. 1 \\
\hline Operator: & Andrew K. Kercher \\
\hline Folder name containing images: & 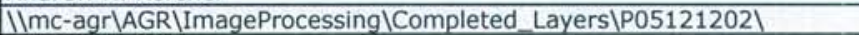 \\
\hline Sample ID: & LEU01-35T-B01 \\
\hline Sample Description: & Baseline TRISO on BWXT kernel composite 69302 \\
\hline Folder name containing processed data: & 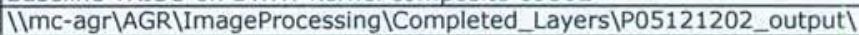 \\
\hline
\end{tabular}

\begin{tabular}{r|c|}
\hline Number of inner pyrocarbon layers analyzed: & 214 \\
\hline Mean of the average IPyC thickness of each particle $(\mu \mathrm{m}):$ & 39.7 \\
\hline Standard deviation in the average IPyC thickness of each particle $(\mu \mathrm{m}):$ & 2.2 \\
\hline
\end{tabular}

\section{Distribution of the average IPyC layer thickness (top binned)}

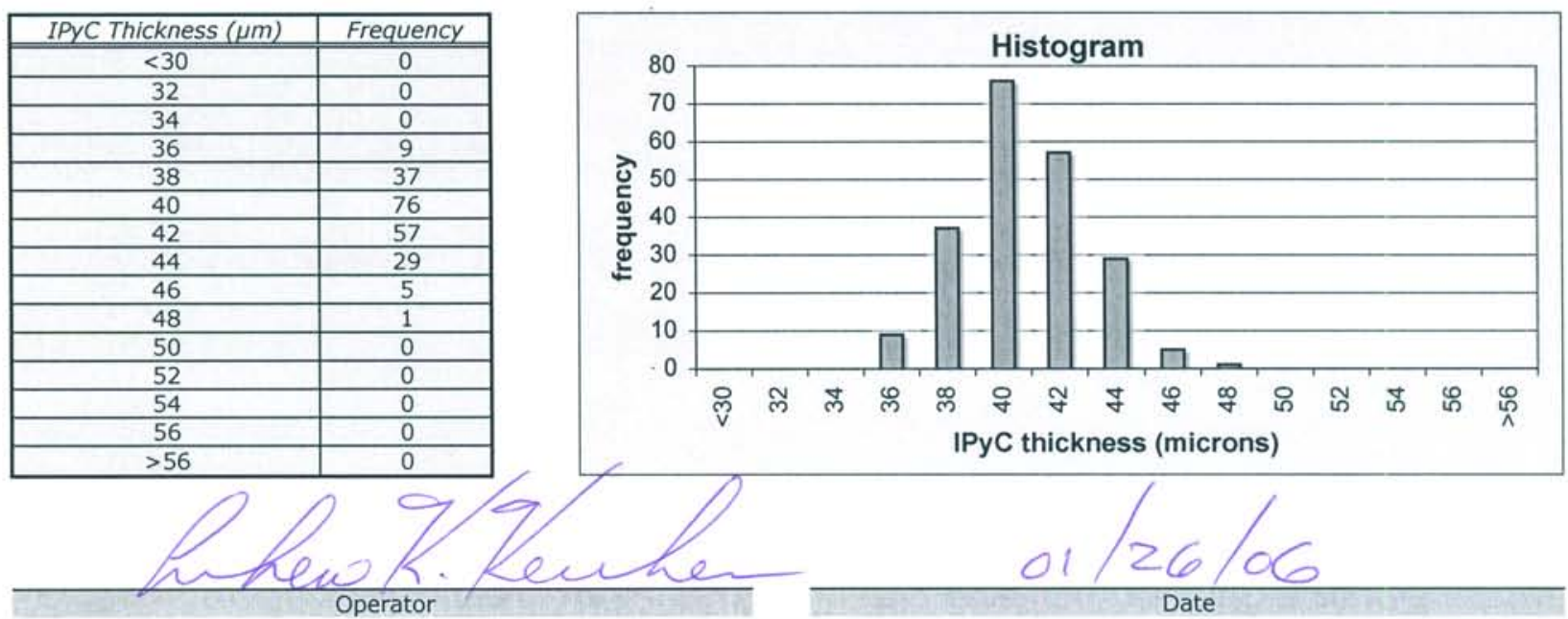


Data Report Form DRF-11C: Measurement of Silicon Carbide Layer Thickness

\begin{tabular}{|c|c|}
\hline Procedure: & AGR-CHAR-DAM-11 Rev. 1 \\
\hline Operator: & Andrew K. Kercher \\
\hline Folder name containing images: & ||mc-agr|AGR|ImageProcessing|Completed_Layers|P05121202| \\
\hline Sample ID: & LEU01-35T-B01 \\
\hline Sample Description: & Baseline TRISO on BWXT kernel composite 69302 \\
\hline Folder name containing processed data: & |Imc-agr|AGR\ImageProcessing|Completed_Layers\P05121202_output| \\
\hline
\end{tabular}

\begin{tabular}{|r|c|}
\hline Number of silicon carbide layers analyzed: & 214 \\
\hline Mean of the average SiC thickness of each particle $(\mu \mathrm{m}):$ & 36.3 \\
\hline Standard deviation in the average SiC thickness of each particle $(\mu \mathrm{m}):$ & 1.0 \\
\hline
\end{tabular}

\section{Distribution of the average SiC layer thickness (top binned)}

\begin{tabular}{|c|c|}
\hline SiC Thickness $(\mu \mathrm{m})$ & Frequency \\
\hline$<25$ & 0 \\
\hline 26 & 0 \\
\hline 27 & 0 \\
\hline 28 & 0 \\
\hline 29 & 0 \\
\hline 30 & 0 \\
\hline 31 & 0 \\
\hline 32 & 0 \\
\hline 33 & 0 \\
\hline 34 & 2 \\
\hline 35 & 23 \\
\hline 36 & 60 \\
\hline 37 & 79 \\
\hline 38 & 42 \\
\hline 39 & 7 \\
\hline 40 & 1 \\
\hline$>40$ & 0 \\
\hline
\end{tabular}
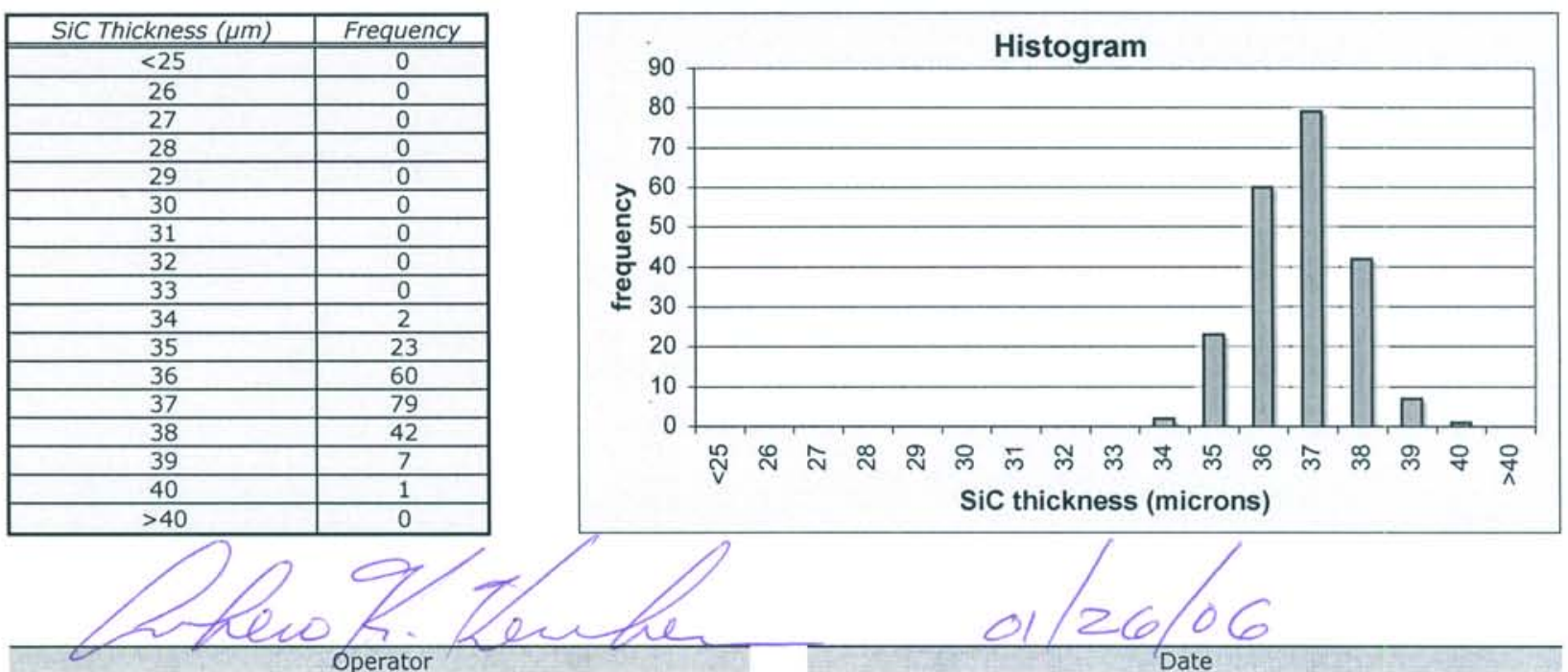


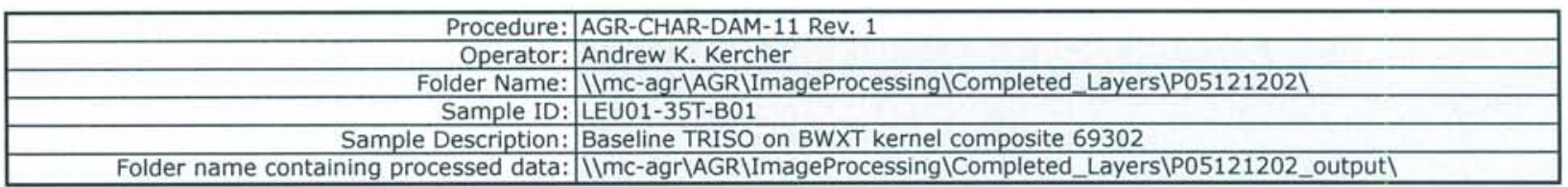

\begin{tabular}{r|r|}
\hline Number of outer pyrocarbon layers analyzed: & 214 \\
\hline Mean of the average OPyC thickness of each particle $(\mu \mathrm{m}):$ & 40.2 \\
\hline Standard deviation in the average OPyC thickness of each particle $(\mu \mathrm{m}):$ & 1.9 \\
\hline
\end{tabular}

\section{Distribution of the average OPyC layer thickness (top binned)}

\begin{tabular}{|c|c|}
\hline OPyC Thickness $(\mu \mathrm{m})$ & Frequency \\
\hline \hline$<20$ & 0 \\
\hline 22 & 0 \\
\hline 24 & 0 \\
\hline 26 & 0 \\
\hline 28 & 0 \\
\hline 30 & 0 \\
\hline 32 & 0 \\
\hline 34 & 0 \\
\hline 36 & 3 \\
\hline 38 & 24 \\
\hline 40 & 76 \\
\hline 42 & 72 \\
\hline 44 & 36 \\
\hline 46 & 3 \\
\hline 48 & 0 \\
\hline 50 & 0 \\
\hline$>50$ & 0 \\
\hline & \\
\hline & \\
\hline
\end{tabular}
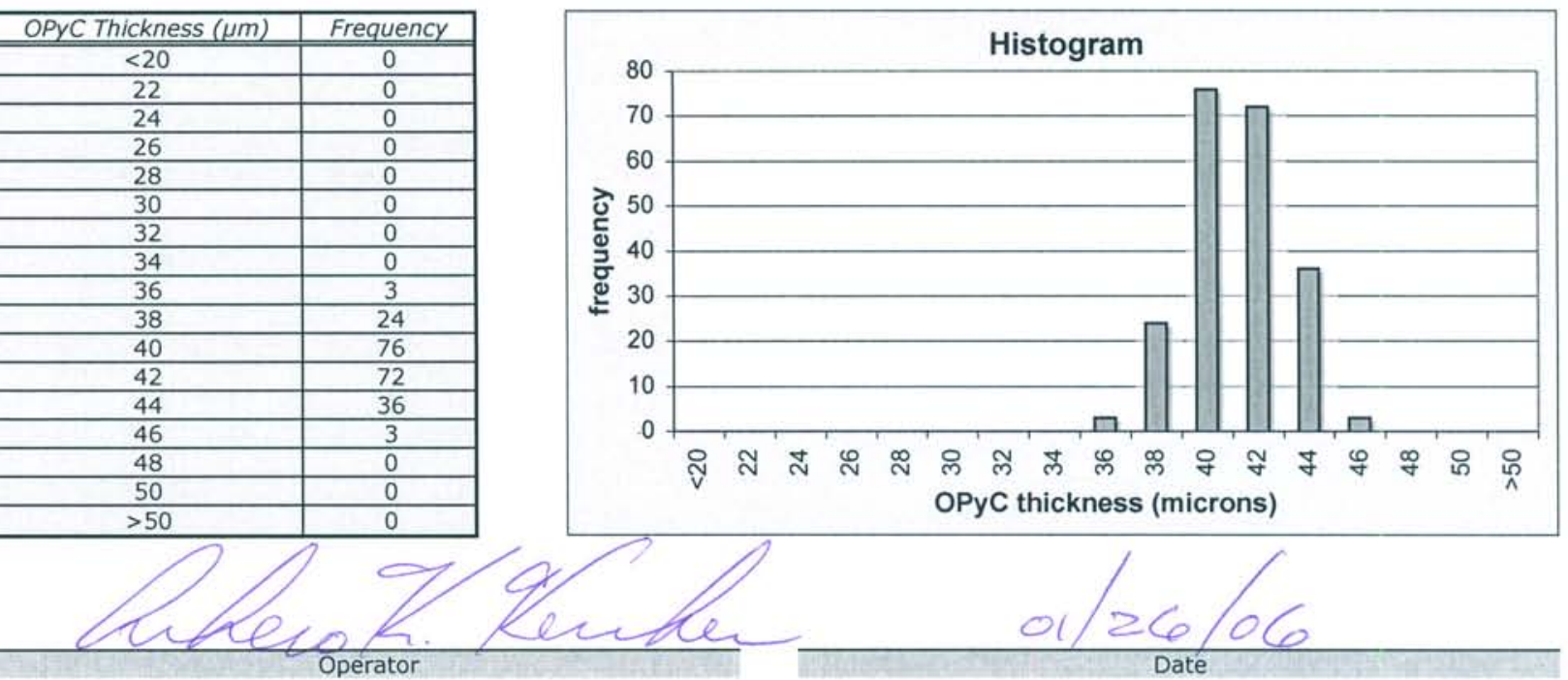
Data Report Form DRF-19: Counting of Particles with Missing OPyC Layer by Visual Inspection

\begin{tabular}{|r|l|}
\hline Procedure: & AGR-CHAR-DAM-19 Rev. 1 \\
\hline Operator: & John Hunn \\
\hline Sample ID: & LEU01-35T-C01 \\
\hline Sample Description: & TRISO on BWXT kernel composite 69302 \\
\hline Filename: & IIMC-agrIAGR\MissingOPyCIX05121201_DRF19R1.xIs \\
\hline
\end{tabular}

Mean average weight/particle $(g): 7.27 \mathrm{E}-04$

Uncertainty in average weight/particle $(g): 2.10 \mathrm{E}-06$

Weight of sample of particles $(\mathrm{g}): 11.357$

Approximate number of particles in sample: 15622

Uncertainty in number of particles in sample: 45

Number of particles with missing OPyC layer: 0

Comments on unusual visual characteristics of OPyC

A small blemish slightly darker than the average OPyC surface was observed on a small fraction of particles (estimated occurance about $0.5 \%$ ).
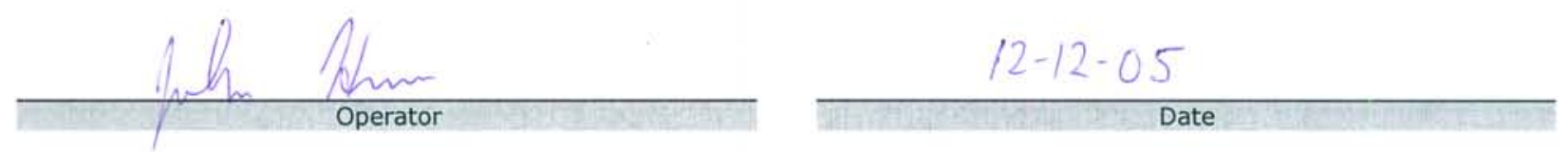
A small dark blemish was observed on less than $0.5 \%$ of the particles from LEU01-35T$\mathrm{C} 01$.

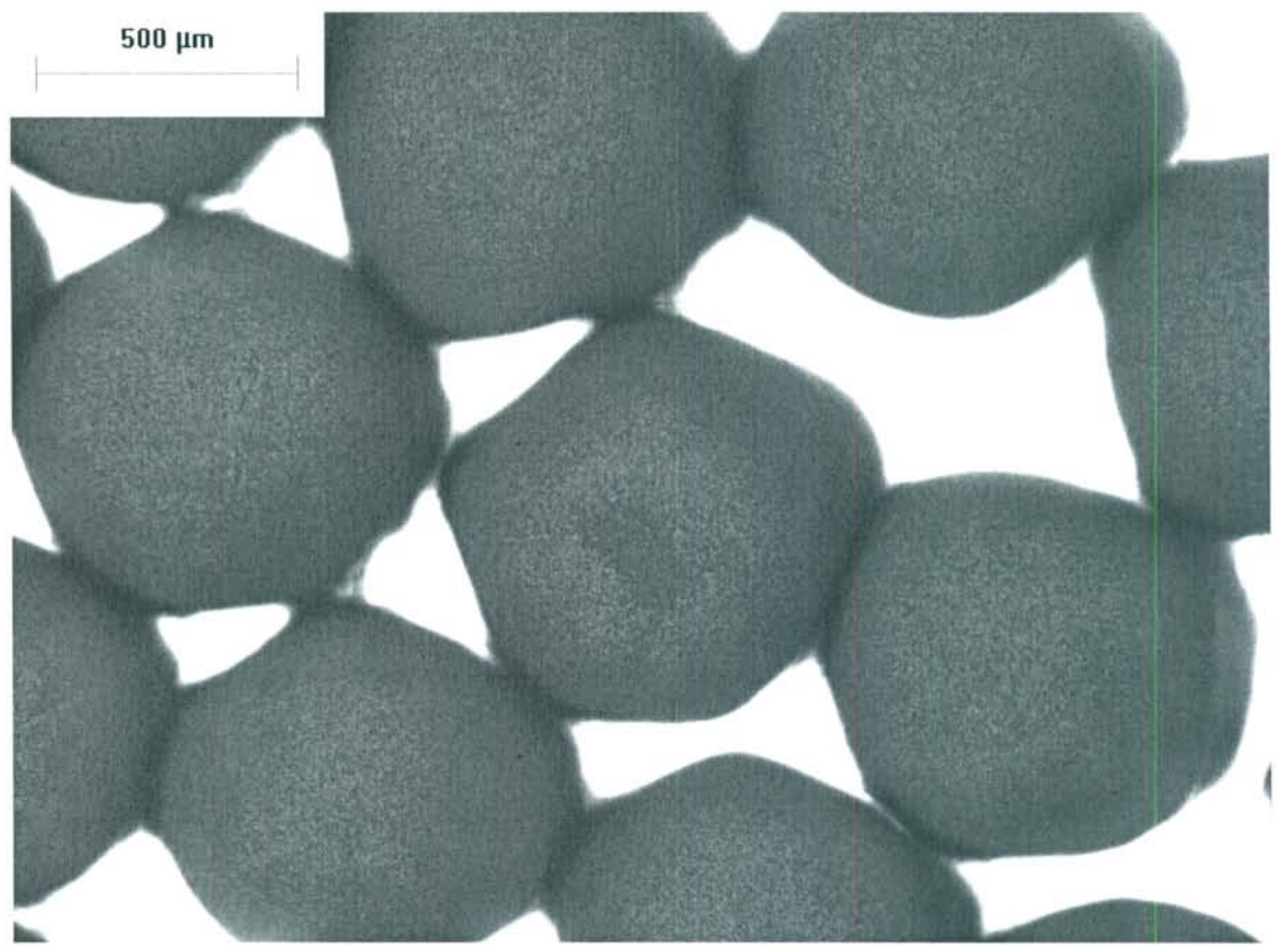




\section{For Information Only}

The information in the remainder of this section reports results of measurements not required by the fuel specification and is provided for information only. 
ORNL/TM-2006/019, Rev. 1

Data Report Form DRF-22: Estimation of Average Particle Weight

Procedure: AGR-CHAR-DAM-22 Rev. 1

\begin{tabular}{|r|l|}
\hline Procedure: & AGR-CHAR-DAM-22 Rev. 1 \\
\hline Operator: & Andy Nelson \\
\hline Particle Lot ID: & LEU 01-35T-D01 \\
\hline Particle Lot Description: & TRISO on BWXT kernel composite 69302 \\
\hline Filename: & IImc-agr\AGR IParticleWeight|W05121201_DRF22R1.xIs \\
\hline
\end{tabular}

\begin{tabular}{|r|c|c|c|c|c|}
\hline & Sample 1 & Sample 2 & Sample 3 & Sample 4 & Sample 5 \\
\hline Weight of particles (g): & $8.52 \mathrm{E}-02$ & $7.79 \mathrm{E}-02$ & $1.07 \mathrm{E}-01$ & $9.47 \mathrm{E}-02$ & $7.91 \mathrm{E}-02$ \\
\hline Number of particles: & 117 & 108 & 147 & 131 & 108 \\
\hline Average weight/particle (g): & $7.28 \mathrm{E}-04$ & $7.21 \mathrm{E}-04$ & $7.30 \mathrm{E}-04$ & $7.23 \mathrm{E}-04$ & $7.32 \mathrm{E}-04$ \\
\hline
\end{tabular}

Mean average weight/particle $(g): 7.27 \mathrm{E}-04$ Uncertainty in mean average weight/particle $(\mathrm{g}): 2.10 \mathrm{E}-06$
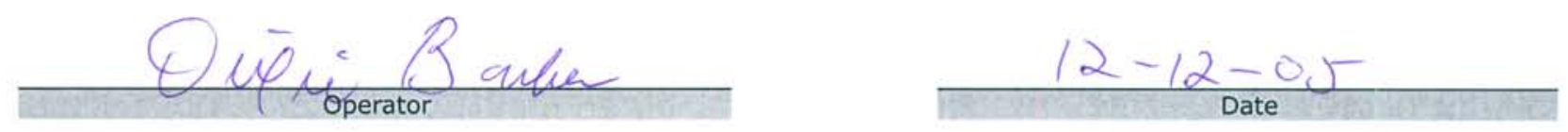

133 
Data Report Form DRF-20: Counting of Particles with SiC Gold Spot Defects by Visual Inspection

\begin{tabular}{|r|l|}
\hline Procedure: & AGR-CHAR-DAM-20 Rev. 1 \\
\hline Operator: & John Hunn \\
\hline Sample ID: & LEU01-35T-C01 \\
\hline Sample Description: & TRISO on BWXT kernel composite 69302 \\
\hline Filename: & IImC-agr|AGR IGoldSpots $\backslash$ G05121201_DRF20R1.xIs \\
\hline
\end{tabular}

Mean average weight/particle (g): $7.27 \mathrm{E}-04$

Uncertainty in average weight/particle $(\mathrm{g}): 2.10 \mathrm{E}-06$

Weight of sample of particles $(\mathrm{g}): 11.357$

Approximate number of particles in sample: 15622

Uncertainty in number of particles in sample: 45

Number of particles with gold spot defects: 17

Comments on unusual visual characteristics of SiC

One particle of the $17 \mathrm{had}$ an unusual crystalline surface.

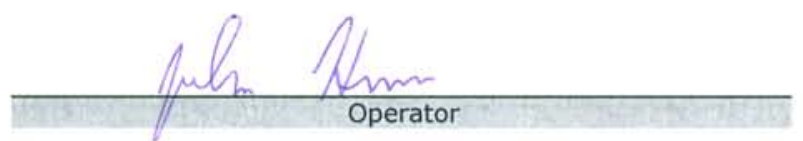

$12-12-05$ 
17/15622 gold spots found. One particle had an unusual crystalline surface.

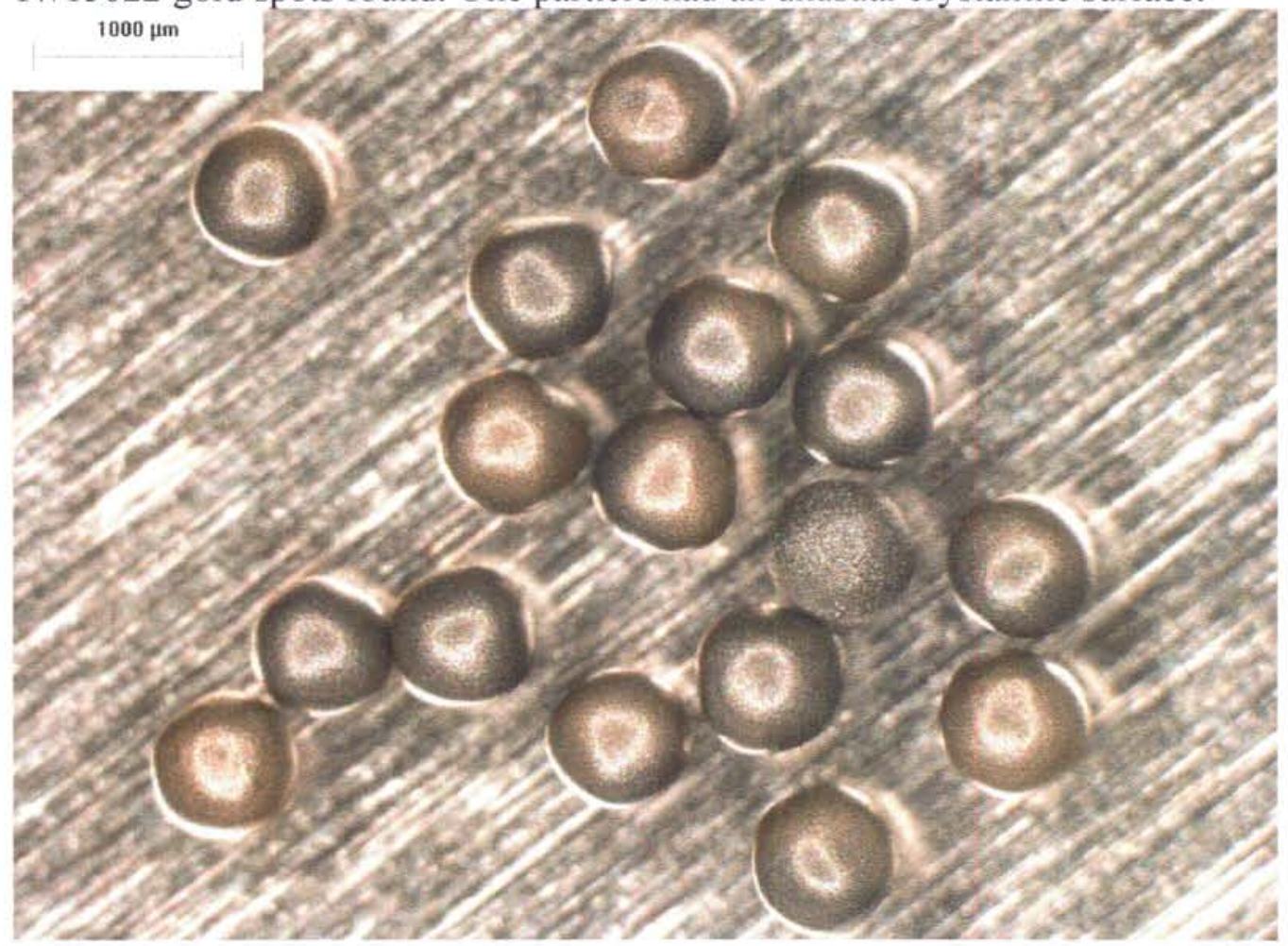

Average appearance of SiC after burnback for LEU01-35T

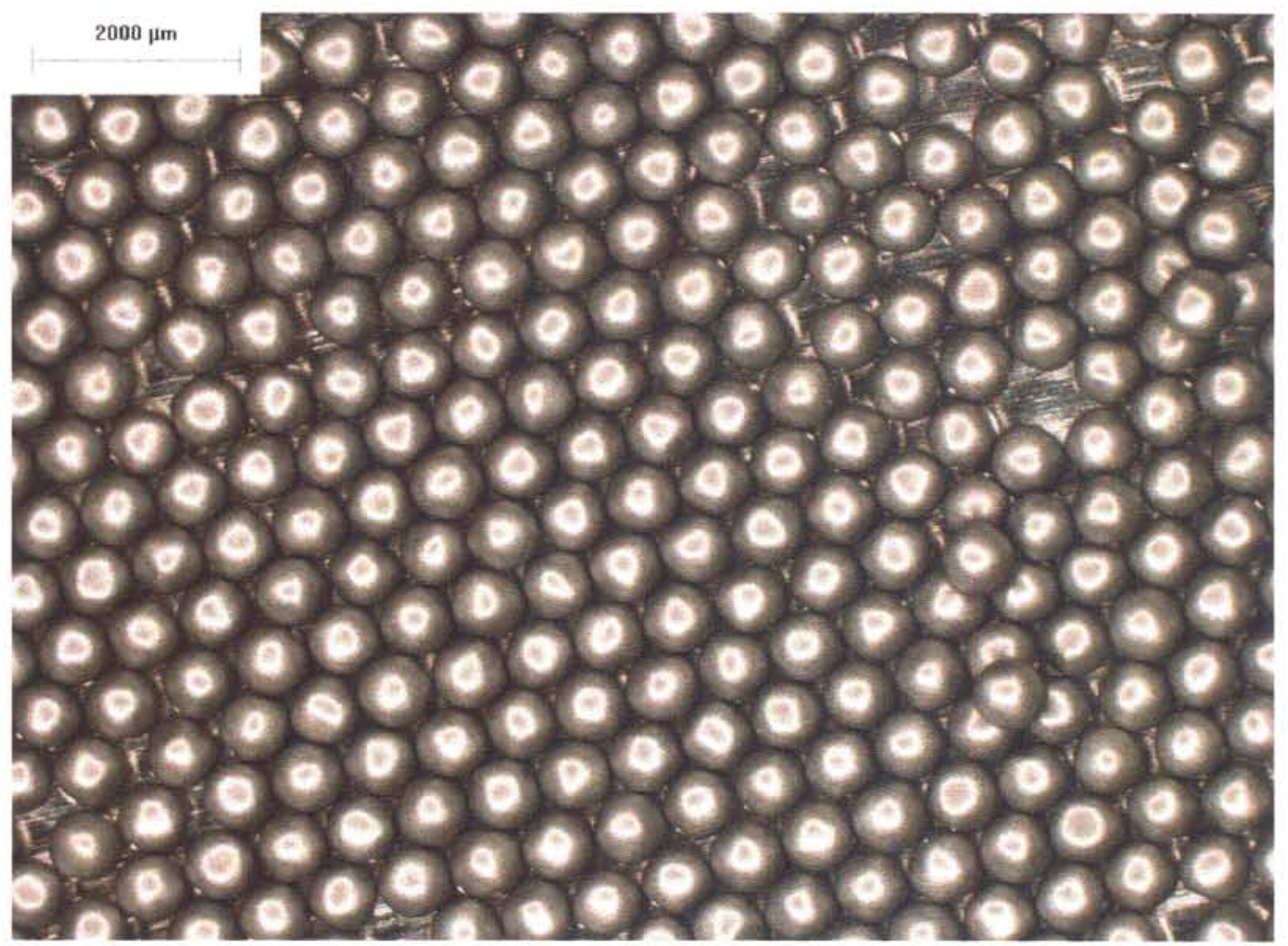




\section{Characterization of third batch of TRISO-coated particles}

This section contains data on LEU01-27T, the third batch of TRISO-coated particles used for the LEU01-46T particle composite. The data was obtained according to product inspection plan AGR-CHAR-PIP-03R2.

Note that some of the carbon deposited for the buffer layer reacted with the kernel to form a uranium carbide layer between the kernel and the buffer. The uranium carbide layer was not included in the measurement of the buffer thickness. The thickness of this carbide layer varied, but was typically around $6 \mu \mathrm{m}$ thick and effectively increased the kernel radius by that amount.

Note that the coating thicknesses of the TRISO-coated particle batch were remeasured after coated particle composite characterization was underway in order to improve the accuracy of the reported values. The original images of the particle cross sections were reanalyzed using an updated version of the image analysis program. This new version utilized a more robust algorithm for identifying the outer boundary of the outer pyrocarbon layer (OPyC). The data in this section shows the new coating thickness values. A record of the original measured values, which were initially used to determine the acceptance of this batch for inclusion in the composite, is included in section 16. Both the old and new coating thickness values for batch LEU01-27T were within the range specified in INL EDF-4380, Rev. 6.

The following pages show the inspection report form (IRF-03). Following IRF-03 are the individual data report forms for the measurements that were performed. Additional data at the end of this section is provided for information only. This batch was determined to satisfy the specifications in section 5.2 of EDF 4380, Rev. 6. 
ORNL/TM-2006/019, Rev. 1

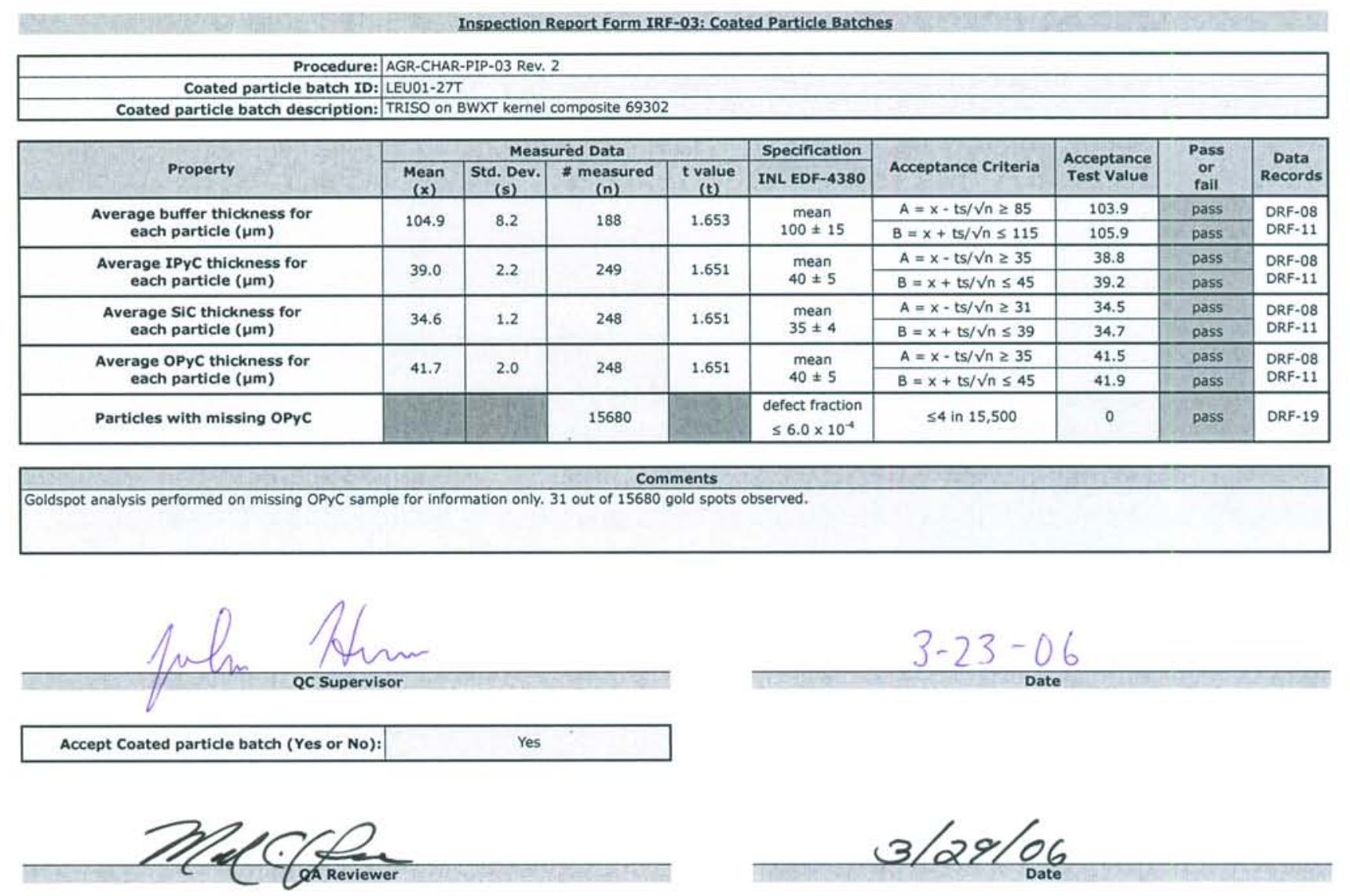

137 
ORNL/TM-2006/019, Rev. 1

\section{Data Report Form DRF-08: Imaging of Coated Particle Cross-sections Using an Optical Microscope System}

\begin{tabular}{|r|r|}
\hline Procedure: & AGR-CHAR-DAM-08 Rev. 2 \\
\hline Operator: & Andrew K. Kercher \\
\hline Sample ID: & LEU01-27T-B01 \\
\hline Sample description: & TRISO on BWXT kernel composite 69302 \\
\hline Mount ID number: & M05121902L \\
\hline Folder name containing images: & IIMc-agrlagr|ImageProcessinglP05122001\P051220101 \\
\hline
\end{tabular}

DMR calibration expiration date: $9 / 8 / 06$ Calibrated pixels/micron: 2.8260

Stage micrometer calibration expiration date: $2 / 17 / 07$

Measured value for $500 \mu \mathrm{m}$ in stage micrometer image $(\mu \mathrm{m}): 500.0$

\begin{tabular}{|c|c|c|c|}
\hline \multicolumn{4}{|c|}{ Polish-down distance $n, m(\mu \mathrm{m})$} \\
\hline 2,2 & 2,8 & 8,2 & 8,8 \\
\hline \hline 382 & $393(2,9)$ & 398 & $413(8,9)$ \\
\hline
\end{tabular}

\begin{tabular}{|c|c|c|c|c|}
\hline \multicolumn{5}{|c|}{ Approximate layer width in polish plane $(\mu \mathrm{m})$} \\
\hline Kernel radius & Buffer & IPyC & SiC & OPyC \\
\hline \hline 177 & 105 & 38 & 38 & 39 \\
\hline
\end{tabular}

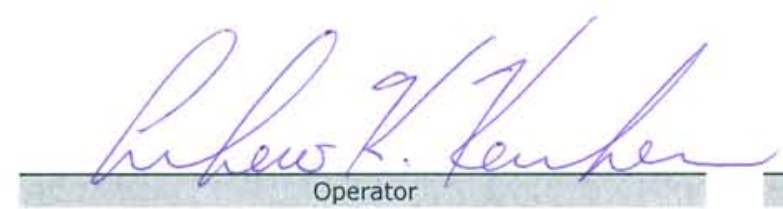

$$
\frac{12 / 20 / 05}{\text { Date }}
$$


ORNL/TM-2006/019, Rev. 1

Data Report Form DRF-08: Imaging of Coated Particle Cross-sections Using an Optical Microscope System

\begin{tabular}{|c|c|}
\hline Procedure: & AGR-CHAR-DAM-08 Rev. 2 \\
\hline Operator: & Andrew K. Kercher \\
\hline Sample ID: & LEU01-27T-B01 \\
\hline Sample description: & TRISO on BWXT kernel composite 69302 \\
\hline Mount ID number: & M05122001L \\
\hline Folder name containing images: & 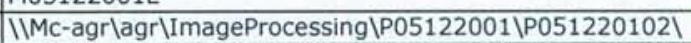 \\
\hline
\end{tabular}

\begin{tabular}{|r|r|}
\hline DMR calibration expiration date: & $9 / 8 / 06$ \\
\hline Calibrated pixels/micron: & 2.8260 \\
\hline Stage micrometer calibration expiration date: & $2 / 17 / 07$ \\
\hline Measured value for $500 \mu \mathrm{m}$ in stage micrometer image $(\mu \mathrm{m}):$ & 500.0 \\
\hline
\end{tabular}

\begin{tabular}{|c|c|c|c|}
\hline \multicolumn{4}{|c|}{ Polish-down distance $n, m(\mu \mathrm{m})$} \\
\hline 2,2 & 2,8 & 8,2 & 8,8 \\
\hline 415 & 409 & 388 & 383 \\
\hline
\end{tabular}

\begin{tabular}{|c|c|c|c|c|}
\hline \multicolumn{5}{|c|}{ Approximate layer width in polish plane $(\mu \mathrm{m})$} \\
\hline Kernel radius & Buffer & IPyC & SiC & OPyC \\
\hline \hline 176 & 99 & 39 & 37 & 42 \\
\hline
\end{tabular}
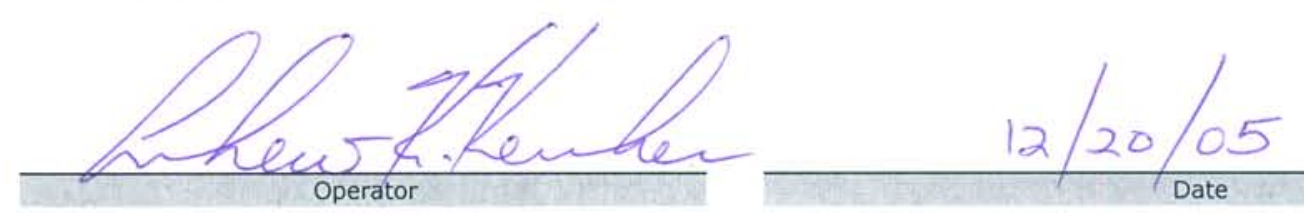
ORNL/TM-2006/019, Rev. 1

Data Report Form DRF-08: Imaging of Coated Particle Cross-sections Using an Optical Microscope System

\begin{tabular}{|r|l|}
\hline Procedure: & AGR-CHAR-DAM-08 Rev. 2 \\
\hline Operator: & Andrew K. Kercher \\
\hline Sample ID: & LEU01-27T-B01 \\
\hline Sample description: & TRISO on BWXT kernel composite 69302 \\
\hline Mount ID number: & M05122002L \\
\hline Folder name containing images: & ImageProcessing\\
P05122001\P051220103\} $\\
{\hline}$
\end{tabular}

DMR calibration expiration date: $9 / 8 / 06$

Calibrated pixels/micron: 2.8260

Stage micrometer calibration expiration date: $2 / 17 / 07$

Measured value for $500 \mu \mathrm{m}$ in stage micrometer image $(\mu \mathrm{m}): 500.0$

\begin{tabular}{|c|c|c|c|}
\hline \multicolumn{4}{|c|}{ Polish-down distance $n, m(\mu \mathrm{m})$} \\
\hline 2,2 & 2,8 & 8,2 & 8,8 \\
\hline \hline 373 & $381(2,9)$ & 358 & $363(8,9)$ \\
\hline
\end{tabular}

\begin{tabular}{|c|c|c|c|c|}
\hline \multicolumn{5}{|c|}{ Approximate layer width in polish plane $(\mu \mathrm{m})$} \\
\hline Kernel radius & Buffer & IPyC & SiC & OPyC \\
\hline \hline 169 & 106 & 40 & 37 & 43 \\
\hline
\end{tabular}
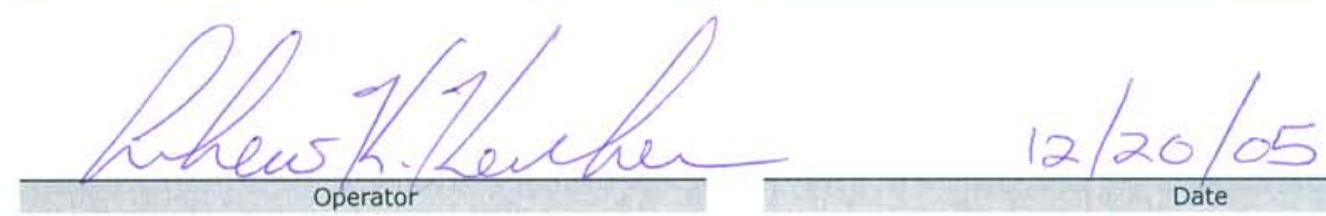


\begin{tabular}{|c|c|}
\hline Procedure: & AGR-CHAR-DAM-11 Rev, 1 \\
\hline Operator: & Andrew K. Kercher \\
\hline Folder name containing images: & |Imc-agr|AGR|ImageProcessing|Completed_Layers\P05122001| \\
\hline Sample ID: & LEU01-27T-B01 \\
\hline Sample Description: & TRISO on BWXT kernel composite 69302 \\
\hline Folder name containing processed data: & 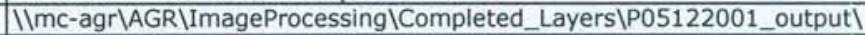 \\
\hline
\end{tabular}

\begin{tabular}{r|r|}
\hline Number of buffer layers analyzed: & 188 \\
\hline Mean of the average buffer thickness of each particle $(\mu \mathrm{m}):$ & 104.9 \\
\hline Standard deviation in the average buffer thickness of each particle $(\mu \mathrm{m}):$ & 8.2 \\
\hline
\end{tabular}

\section{Distribution of the average buffer layer thickness (top binned)}

\begin{tabular}{|c|c|}
\hline Buffer Thickness $(\mu \mathrm{m})$ & Frequency \\
\hline \hline$<55$ & 0 \\
\hline 60 & 0 \\
\hline 65 & 0 \\
\hline 70 & 0 \\
\hline 75 & 0 \\
\hline 80 & 0 \\
\hline 85 & 0 \\
\hline 90 & 6 \\
\hline 95 & 13 \\
\hline 100 & 39 \\
\hline 105 & 36 \\
\hline 110 & 48 \\
\hline 115 & 25 \\
\hline 120 & 14 \\
\hline 125 & 5 \\
\hline$>125$ & 2 \\
\hline
\end{tabular}
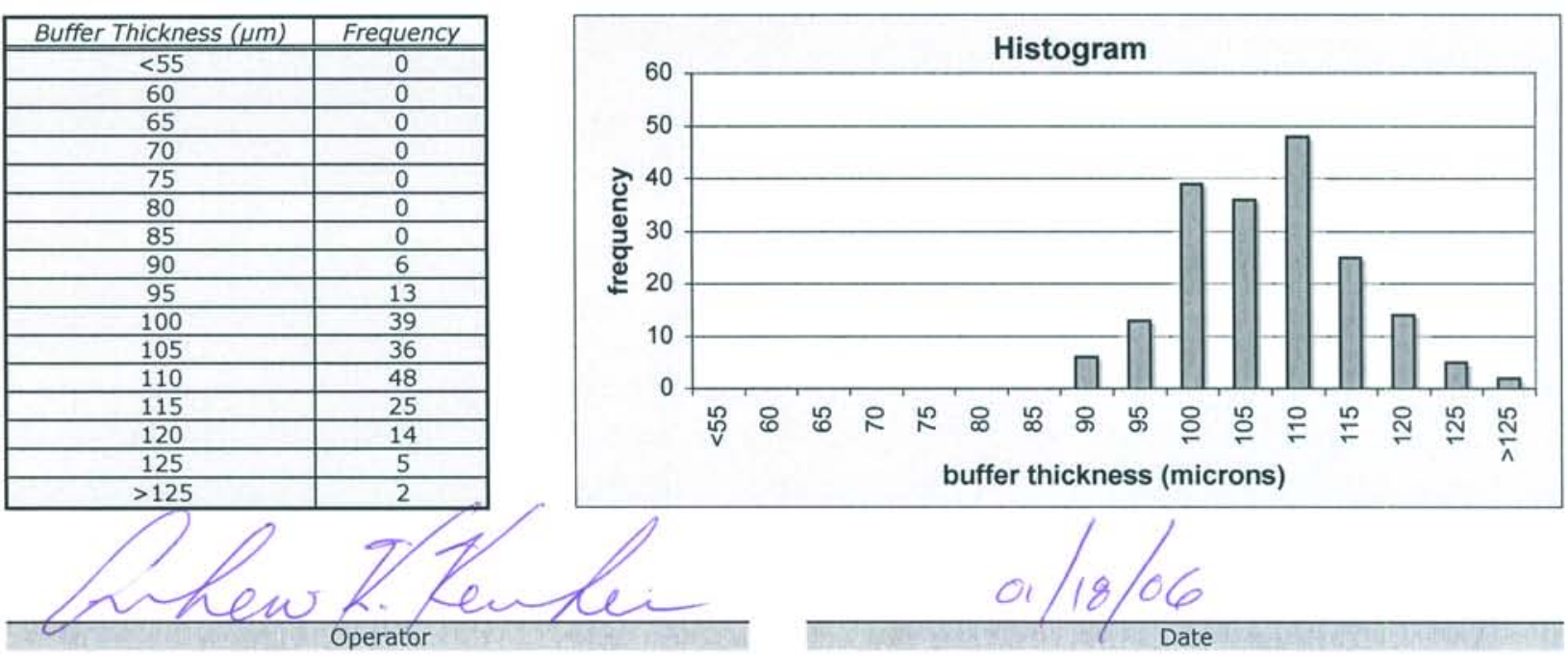


\begin{tabular}{|c|c|}
\hline Procedure: & AGR-CHAR-DAM-11 Rev. 1 \\
\hline Operator: & Andrew K. Kercher \\
\hline Folder name containing images: & IImc-agr|AGR\ImageProcessing|Completed_Layers\P05122001| \\
\hline Sample ID: & LEU01-27T-B01 \\
\hline Sample Description: & TRISO on BWXT kernel composite 69302 \\
\hline Folder name containing processed data: & |Imc-agr|AGR\ImageProcessing|Completed_Layers\P05122001_output| \\
\hline
\end{tabular}

\begin{tabular}{|r|r|}
\hline Number of inner pyrocarbon layers analyzed: & 249 \\
\hline Mean of the average IPyC thickness of each particle $(\mu \mathrm{m}):$ & 39.0 \\
\hline Standard deviation in the average IPyC thickness of each particle $(\mu \mathrm{m}):$ & 2.2 \\
\hline
\end{tabular}

\section{Distribution of the average IPyC layer thickness (top binned)}

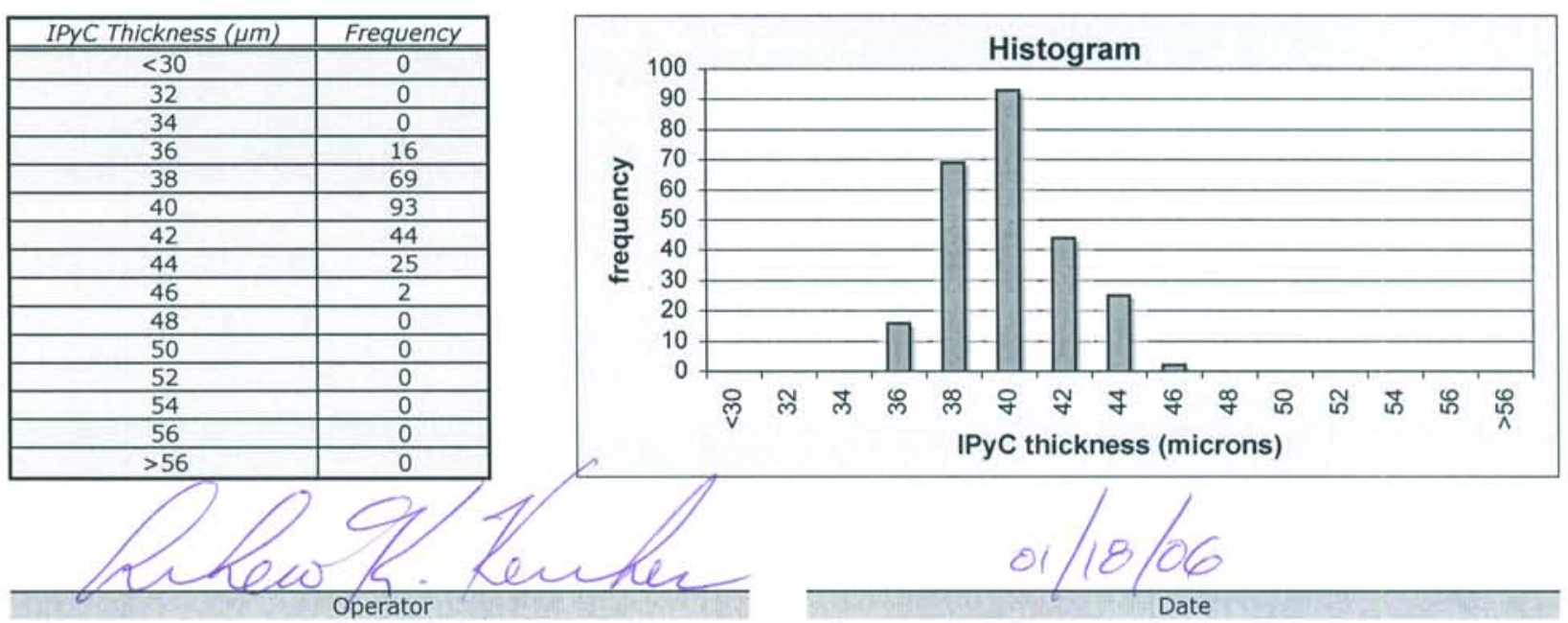


Data Report Form DRF-11C: Measurement of Silicon Carbide Layer Thickness

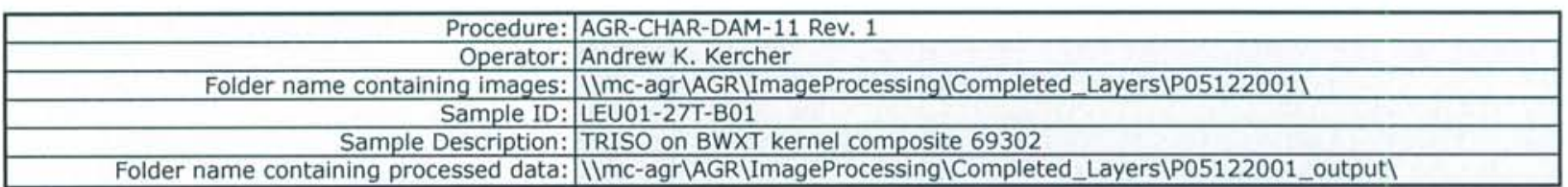

\begin{tabular}{|r|c|}
\hline Number of silicon carbide layers analyzed: & 248 \\
\hline Mean of the average SiC thickness of each particle $(\mu \mathrm{m}):$ & 34.6 \\
\hline Standard deviation in the average SiC thickness of each particle $(\mu \mathrm{m}):$ & 1.2 \\
\hline
\end{tabular}

\section{Distribution of the average SiC layer thickness (top binned)}

\begin{tabular}{|c|c|}
\hline SiC Thickness $(\mu \mathrm{m})$ & Frequency \\
\hline$<25$ & 0 \\
\hline 26 & 0 \\
\hline 27 & 0 \\
\hline 28 & 0 \\
\hline 29 & 2 \\
\hline 30 & 0 \\
\hline 31 & 0 \\
\hline 32 & 1 \\
\hline 33 & 12 \\
\hline 34 & 46 \\
\hline 35 & 94 \\
\hline 36 & 74 \\
\hline 37 & 17 \\
\hline 38 & 1 \\
\hline 39 & 1 \\
\hline 40 & 0 \\
\hline$>40$ & 0 \\
\hline 7 & \\
\hline
\end{tabular}
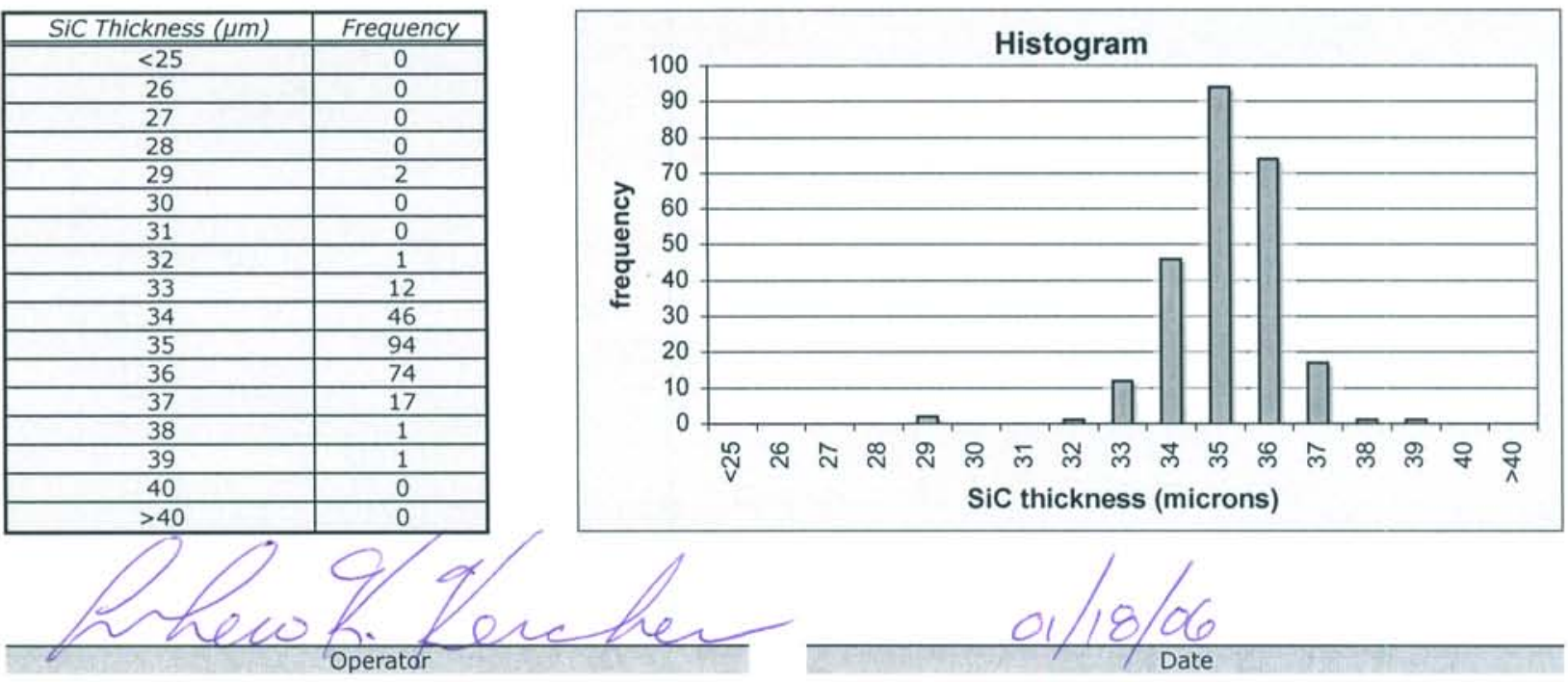
Data Report Form DRF-11D: Measurement of Outer Pyrocarbon Layer Thickness

\begin{tabular}{|c|c|}
\hline Procedure: & AGR-CHAR-DAM-11 Rev. 1 \\
\hline Operator: & Andrew K. Kercher \\
\hline Folder Name: & 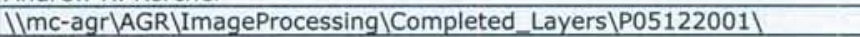 \\
\hline Sample ID: & LEU01-27T-B01 \\
\hline Sample Description: & TRISO on BWXT kernel composite 69302 \\
\hline Folder name containing processed data: & 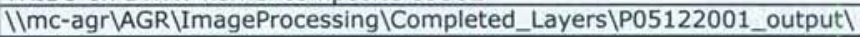 \\
\hline
\end{tabular}

\begin{tabular}{r|c|}
\hline Number of outer pyrocarbon layers analyzed: & 248 \\
\hline Mean of the average OPyC thickness of each particle $(\mu \mathrm{m}):$ & 41.7 \\
\hline Standard deviation in the average OPyC thickness of each particle $(\mu \mathrm{m}):$ & 2.0 \\
\hline
\end{tabular}

\section{Distribution of the average OPyC layer thickness (top binned)}

\begin{tabular}{|c|c|}
\hline OPyC Thickness $(\mu \mathrm{m})$ & Frequency \\
\hline \hline$<20$ & 0 \\
\hline 22 & 0 \\
\hline 24 & 0 \\
\hline 26 & 0 \\
\hline 28 & 0 \\
\hline 30 & 0 \\
\hline 32 & 0 \\
\hline 34 & 0 \\
\hline 36 & 0 \\
\hline 38 & 7 \\
\hline 40 & 46 \\
\hline 42 & 81 \\
\hline 44 & 85 \\
\hline 46 & 24 \\
\hline 48 & 4 \\
\hline 50 & 1 \\
\hline$>50$ & 0 \\
\hline & \\
\hline
\end{tabular}
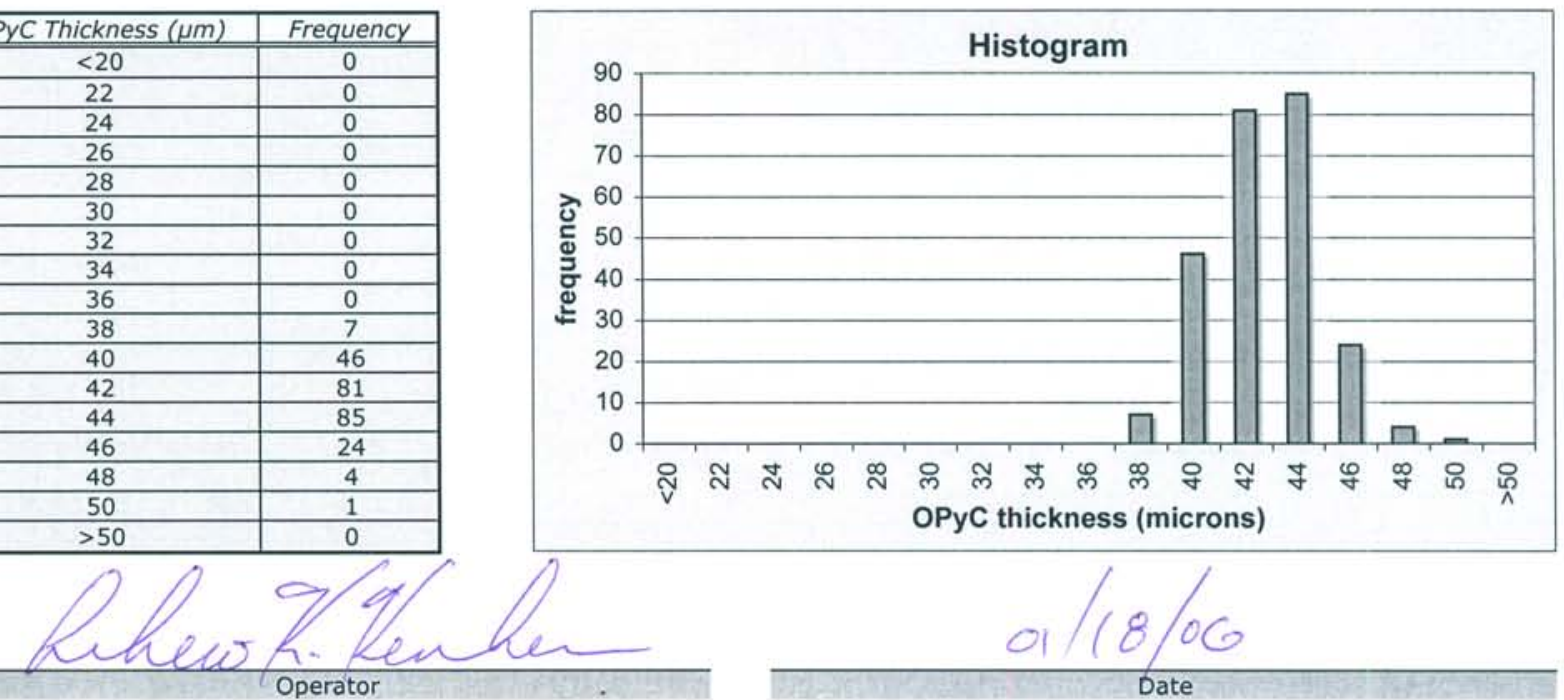
Data Report Form DRF-19: Counting of Particles with Missing OPyC Layer by Visual Inspection

\begin{tabular}{|r|l|}
\hline Procedure: & AGR-CHAR-DAM-19 Rev. 1 \\
\hline Operator: & John Hunn \\
\hline Sample ID: & LEU01-27T-C01 \\
\hline Sample Description: & TRISO on BWXT kernel composite 69302 \\
\hline Filename: & IImC-agr\AGR\MissingOPyCIX05121901_DRF19R1.xIs \\
\hline
\end{tabular}

\begin{tabular}{|r|l|}
\hline Mean average weight/particle $(\mathrm{g}):$ & $7.25 \mathrm{E}-04$ \\
\hline Uncertainty in average weight/particle $(\mathrm{g}):$ & $1.34 \mathrm{E}-06$ \\
\hline Weight of sample of particles $(\mathrm{g}):$ & 11.368 \\
\hline Approximate number of particles in sample: & 15680 \\
\hline Uncertainty in number of particles in sample: & 29 \\
\hline
\end{tabular}

Number of particles with missing OPyC layer: 0

Comments on unusual visual characteristics of OPyC

SEM was performed on dark blemishes commonly observed on a small fraction of particles during analysis for missing OPyC. These appear to be caused by particle to particle contact.
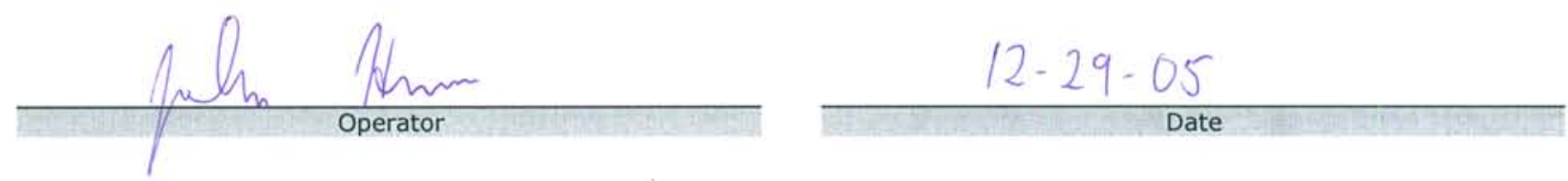
Dark blemishes observed on OPyC surface on a small fraction of particles appear to be caused by temporary particle to particle contact when observed by SEM.

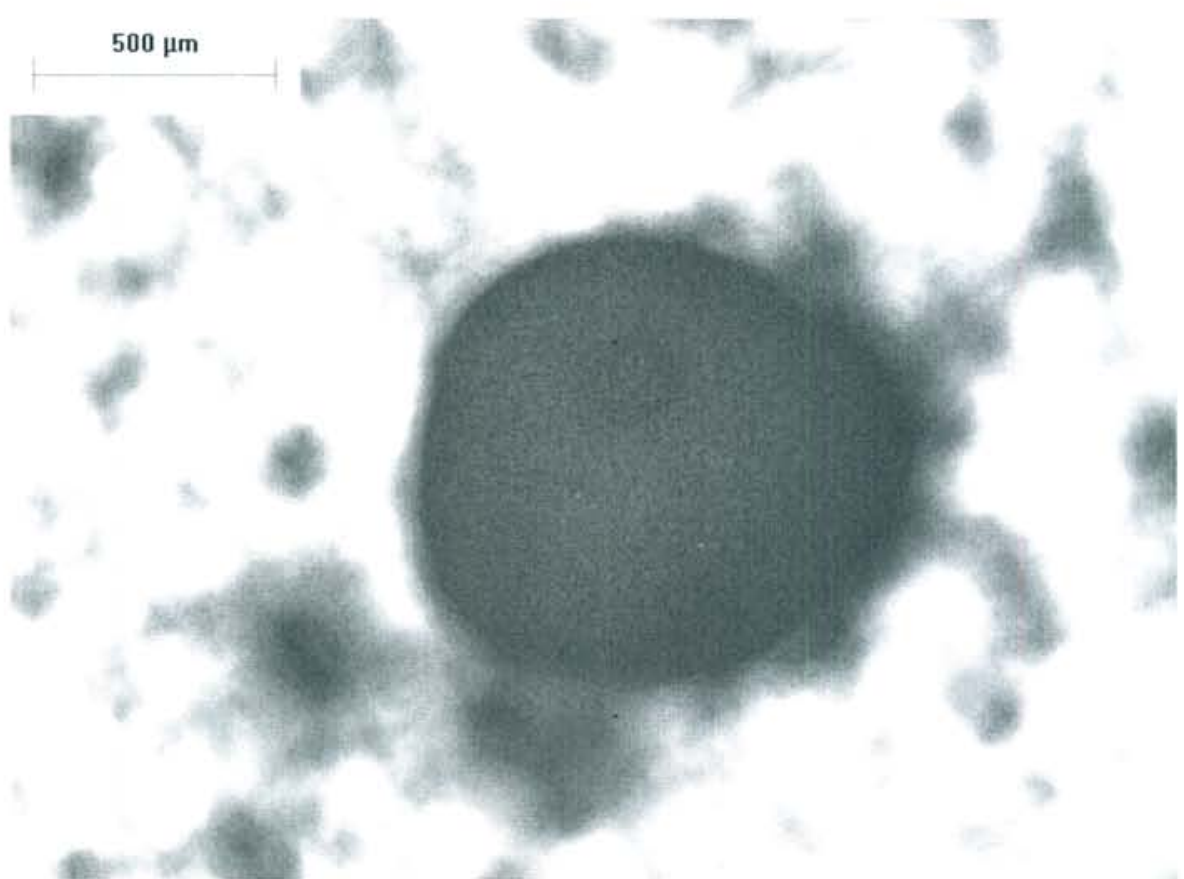

Figure 1. Small dark blemish observed under optical stereo microscope during inspection for missing OPyC.

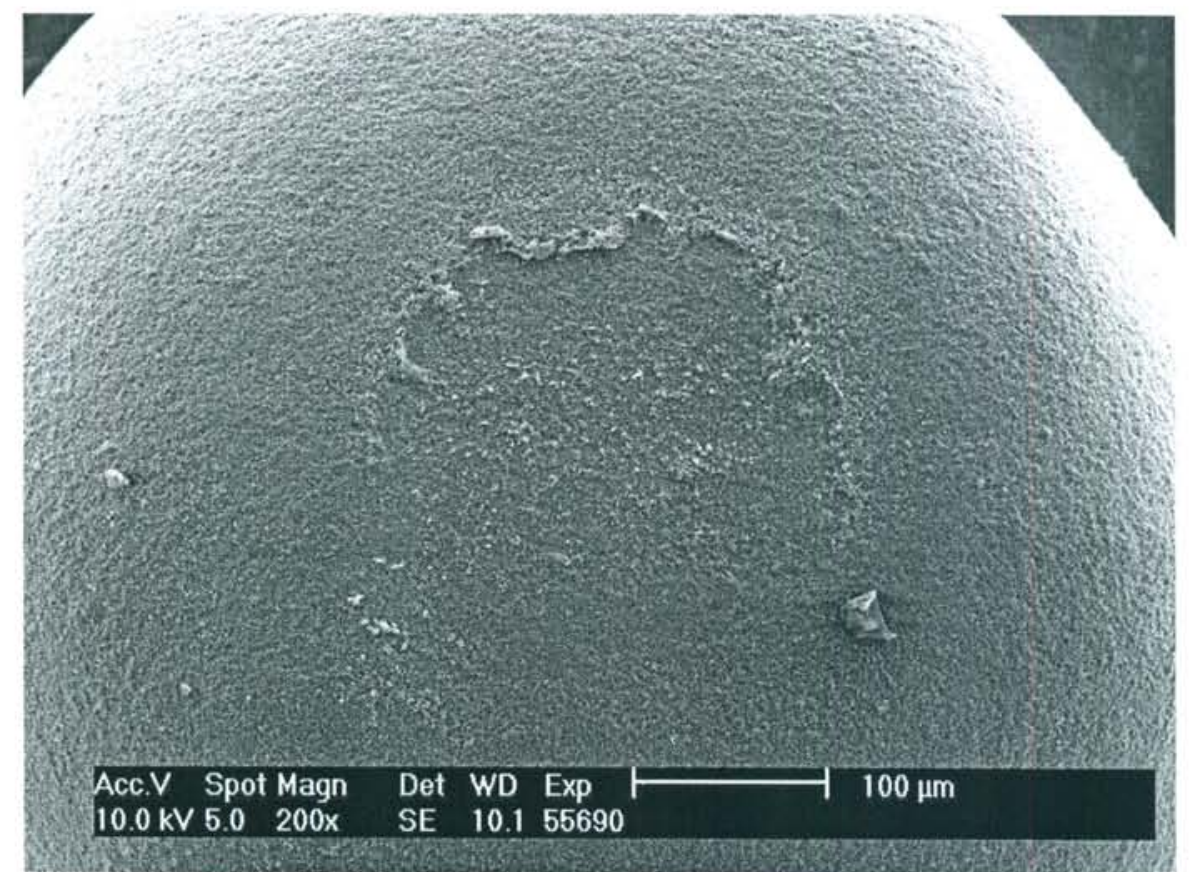

Figure 2. SEM image of blemish. 


\section{For Information Only}

The information in the remainder of this section reports results of measurements not required by the fuel specification and is provided for information only. 
Data Report Form DRF-22: Estimation of Average Particle Weight

\begin{tabular}{|r|r|}
\hline Procedure: & AGR-CHAR-DAM-22 Rev. 1 \\
\hline Operator: & Dixie Barker \\
\hline Particle Lot ID: & LEU01-27T-D01 \\
\hline Particle Lot Description: & TRISO on BWXT kernel composite 69302 \\
\hline Filename: & IImC-agr\AGR\ParticleWeightIW05120201_DRF22R1.xIs \\
\hline
\end{tabular}

\begin{tabular}{|r|c|c|c|c|c|}
\hline & Sample 1 & Sample 2 & Sample 3 & Sample 4 & Sample 5 \\
\hline Weight of particles (g): & $6.72 \mathrm{E}-02$ & $9.72 \mathrm{E}-02$ & $1.09 \mathrm{E}-01$ & $1.02 \mathrm{E}-01$ & $7.93 \mathrm{E}-02$ \\
\hline Number of particles: & 93 & 134 & 150 & 140 & 110 \\
\hline Average weight/particle (g): & $7.23 \mathrm{E}-04$ & $7.25 \mathrm{E}-04$ & $7.27 \mathrm{E}-04$ & $7.28 \mathrm{E}-04$ & $7.21 \mathrm{E}-04$ \\
\hline
\end{tabular}

Mean average weight/particle $(g): 7.25 \mathrm{E}-04$

Uncertainty in mean average weight/particle $(\mathrm{g}): 1.34 \mathrm{E}-06$

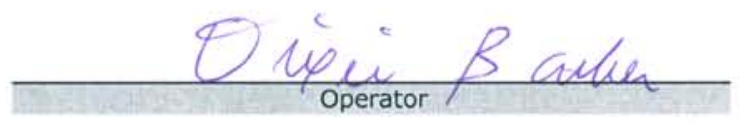

$\frac{12-2-05}{\text { Date }}$


Data Report Form DRF-20: Counting of Particles with SiC Gold Spot Defects by Visual Inspection

\begin{tabular}{|c|c|}
\hline Procedure: & AGR-CHAR-DAM-20 Rev. 1 \\
\hline Operator: & John Hunn \\
\hline Sample ID: & LEU01-27T-C01 \\
\hline Sample Description: & TRISO on BWXT kernel composite 69302 \\
\hline Filename: & IImc-agr|AGR\GoldSpots\G05122001_DRF20R1.xls \\
\hline
\end{tabular}

\begin{tabular}{|r|l|}
\hline Mean average weight/particle $(\mathrm{g}):$ & $7.25 \mathrm{E}-04$ \\
\hline Uncertainty in average weight/particle $(\mathrm{g}):$ & $1.34 \mathrm{E}-06$ \\
\hline Weight of sample of particles $(\mathrm{g}):$ & 11.368 \\
\hline Approximate number of particles in sample: & 15680 \\
\hline Uncertainty in number of particles in sample: & 29 \\
\hline
\end{tabular}

Number of particles with gold spot defects: 31

\section{Comments on unusual visual characteristics of SiC}

Image of goldspots: P0512200201.

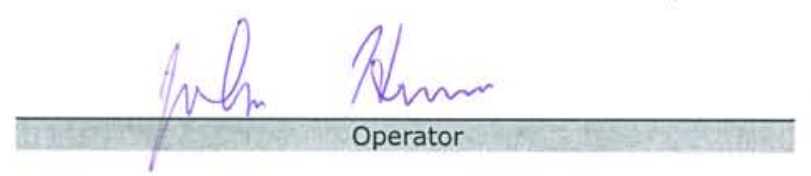

$$
12-20-05
$$


$31 / 15680$ gold spots found.

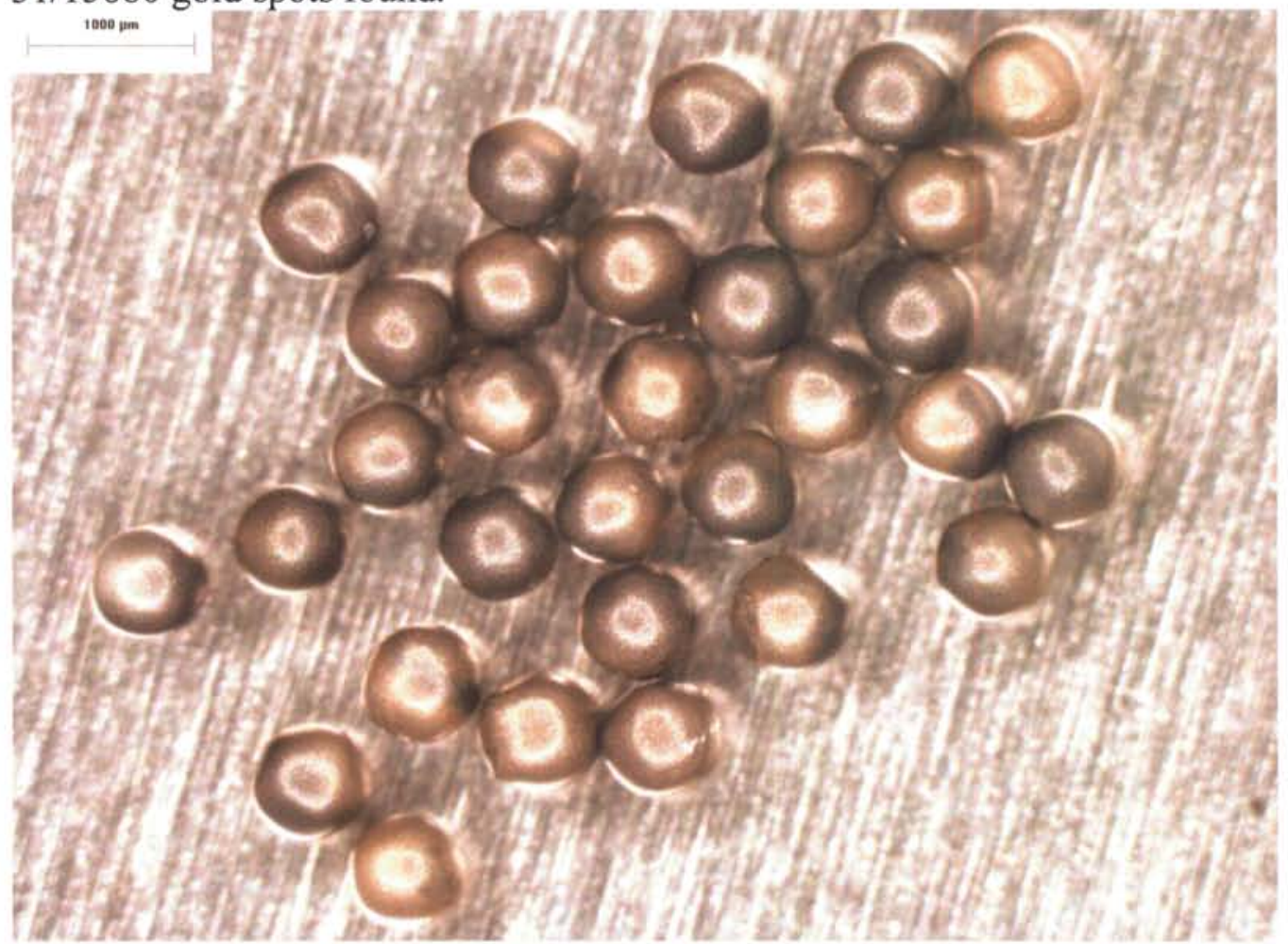

Average appearance of $\mathrm{SiC}$ after burnback for LEU01-27T $2000 \mu \mathrm{m}$

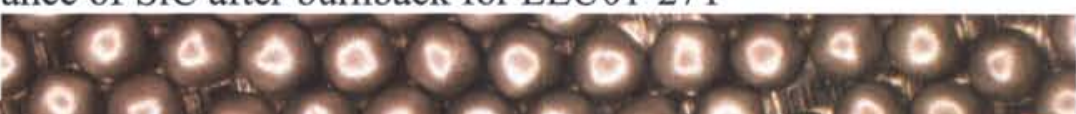

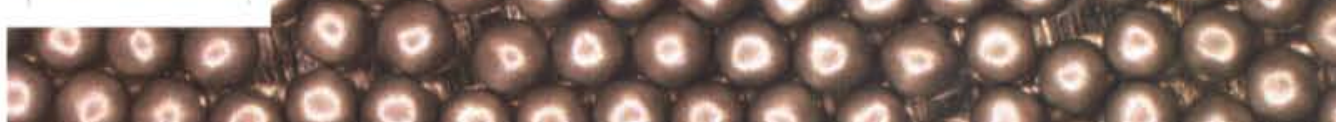

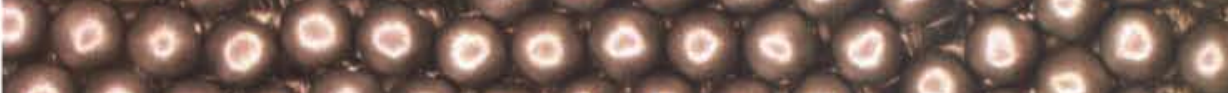

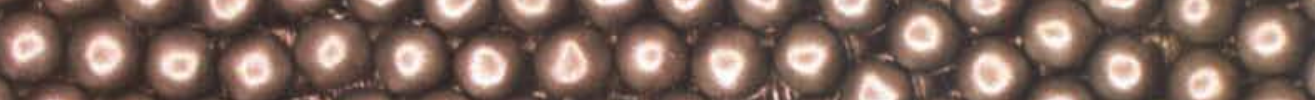

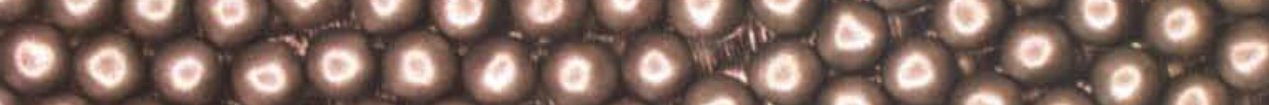

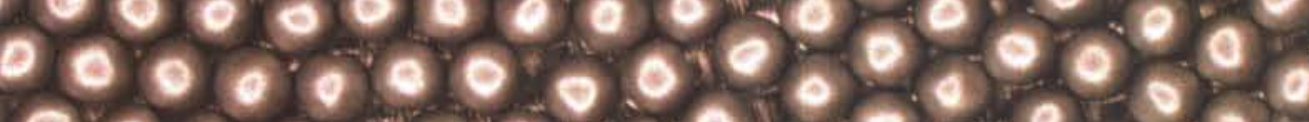

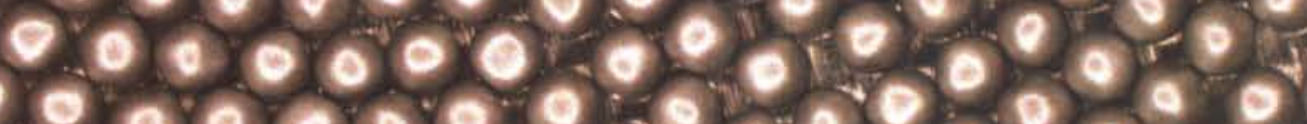

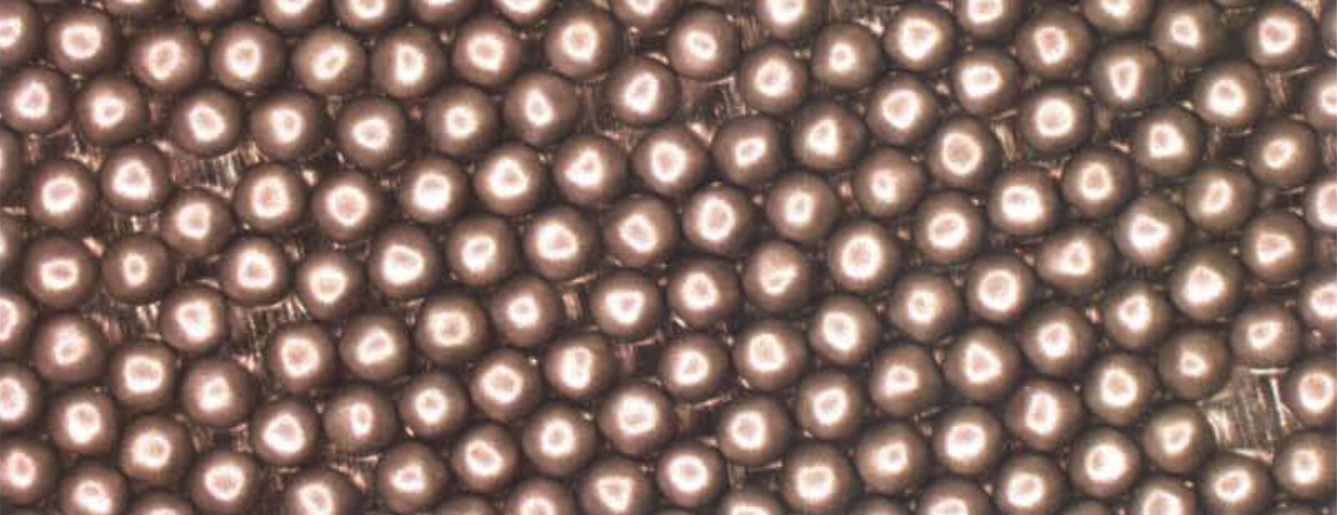




\section{Characterization of fourth batch of TRISO-coated particles}

This section contains data on LEU01-45T, the fourth batch of TRISO-coated particles used for the LEU01-46T particle composite. The data was obtained according to product inspection plan AGR-CHAR-PIP-03R2.

Note that some of the carbon deposited for the buffer layer reacted with the kernel to form a uranium carbide layer between the kernel and the buffer. The uranium carbide layer was not included in the measurement of the buffer thickness. The thickness of this carbide layer varied, but was typically around $6 \mu \mathrm{m}$ thick and effectively increased the kernel radius by that amount.

Note that the coating thicknesses of the TRISO-coated particle batch were remeasured after coated particle composite characterization was underway in order to improve the accuracy of the reported values. The original images of the particle cross sections were reanalyzed using an updated version of the image analysis program. This new version utilized a more robust algorithm for identifying the outer boundary of the outer pyrocarbon layer (OPyC). The data in this section shows the new coating thickness values. A record of the original measured values, which were initially used to determine the acceptance of this batch for inclusion in the composite, is included in section 16. Both the old and new coating thickness values for batch LEU01-45T were within the range specified in INL EDF-4380, Rev. 6.

The following pages show the inspection report form (IRF-03). Following IRF-03 are the individual data report forms for the measurements that were performed. Additional data at the end of this section is provided for information only. This batch was determined to satisfy the specifications in section 5.2 of EDF 4380, Rev. 6. 
ORNL/TM-2006/019, Rev. 1

Inspection Report Form IRF-03: Coated Particle Batches

Procedure: AGR-CHAR-PIP-03 ReV. 2

Coated particle batch ID: LEU 01-45T

Coated particle batch description: TRISO on BWXT kernel composite 69302

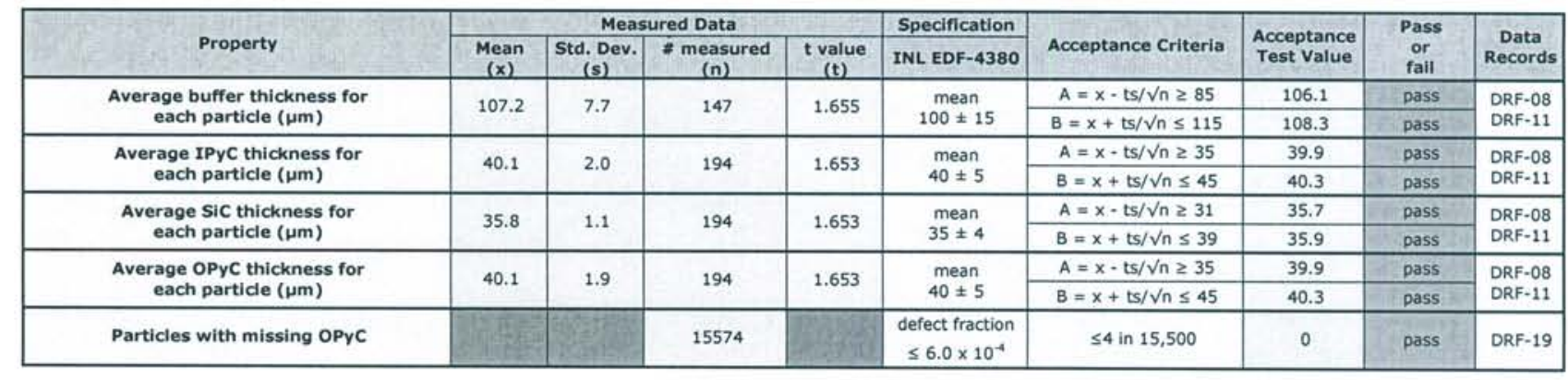

Goldspot analysis performed on missing OPYC sample for information only. 3 out of 15574 gold spots observed.

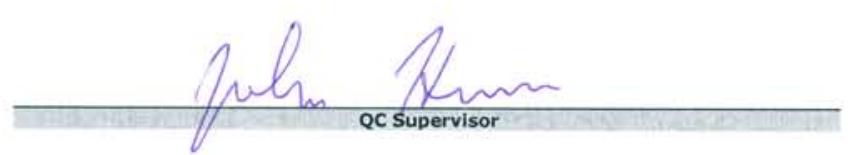

Accept Coated particle batch (Yes or No): Yes.

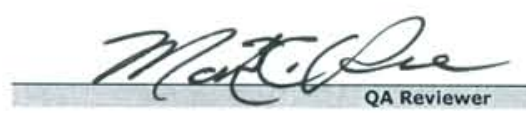

$$
3-23-06
$$

$3 / 24 / 86$

152 
ORNL/TM-2006/019, Rev. 1

Data Report Form DRF-08: Imaging of Coated Particle Cross-sections Using an Optical Microscope System

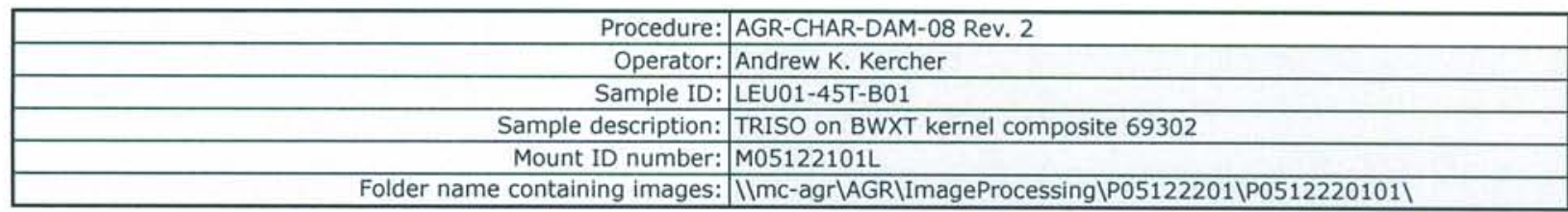

DMR calibration expiration date: $9 / 8 / 06$ Calibrated pixels/micron: 2.8260

Stage micrometer calibration expiration date: 2/17/07

Measured value for $500 \mu \mathrm{m}$ in stage micrometer image $(\mu \mathrm{m}): 500.4$

\begin{tabular}{|c|c|c|c|}
\hline \multicolumn{4}{|c|}{ Polish-down distance $\mathrm{n}, \mathrm{m}(\mu \mathrm{m})$} \\
\hline 2,2 & 2,8 & 8,2 & 8,8 \\
\hline \hline 372 & 372 & 365 & 363 \\
\hline
\end{tabular}

\begin{tabular}{|c|c|c|c|c|}
\hline \multicolumn{5}{|c|}{ Approximate layer width in polish plane $(\mu \mathrm{m})$} \\
\hline Kernel radius & Buffer & IPyC & SiC & OPyC \\
\hline \hline 169 & 110 & 41 & 37 & 36 \\
\hline
\end{tabular}
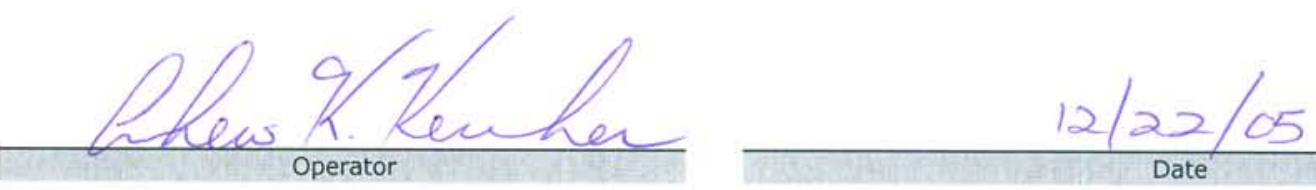
ORNL/TM-2006/019, Rev. 1

Data Report Form DRF-08: Imaging of Coated Particle Cross-sections Using an Optical Microscope System

\begin{tabular}{|r|l|}
\hline Procedure: & AGR-CHAR-DAM-08 Rev. 2 \\
\hline Operator: & Andrew K. Kercher \\
\hline Sample ID: & LEU01-45T-B01 \\
\hline Sample description: & TRISO on BWXT kernel composite 69302 \\
\hline Mount ID number: & M05122102L \\
\hline Folder name containing images: & IIMC-agr\AGR\ImageProcessing\P05122201\P0512220102 \\
\hline
\end{tabular}

DMR calibration expiration date: $9 / 8 / 06$

Calibrated pixels/micron: 2.8260

Stage micrometer calibration expiration date: $2 / 17 / 07$

Measured value for $500 \mu \mathrm{m}$ in stage micrometer image $(\mu \mathrm{m}): 500.4$

\begin{tabular}{|c|c|c|c|}
\hline \multicolumn{4}{|c|}{ Polish-down distance $n, m(\mu \mathrm{m})$} \\
\hline 2,2 & 2,8 & 8,2 & 8,8 \\
\hline \hline 370 & 387 & 367 & 372 \\
\hline
\end{tabular}

\begin{tabular}{|c|c|c|c|c|}
\hline \multicolumn{5}{|c|}{ Approximate layer width in polish plane $(\mu \mathrm{m})$} \\
\hline Kernel radius & Buffer & IPyC & SiC & OPyC \\
\hline \hline 176 & 100 & 39 & 38 & 39 \\
\hline
\end{tabular}

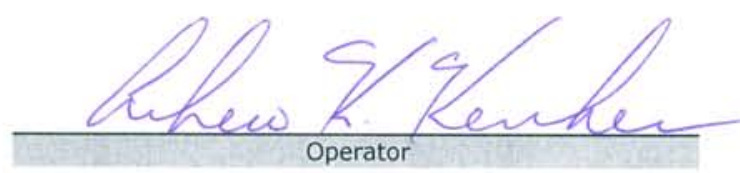

$$
12 / 22 / 05
$$




\section{ORNL/TM-2006/019, Rev. 1}

Data Report Form DRF-08: Imaging of Coated Particle Cross-sections Using an Optical Microscope System

\begin{tabular}{|r|l|}
\hline Procedure: & AGR-CHAR-DAM-08 Rev. 2 \\
\hline Operator: & Andrew K. Kercher \\
\hline Sample ID: & LEU01-45T-B01 \\
\hline Sample description: & TRISO on BWXT kernel composite 69302 \\
\hline Mount ID number: & M05122103L \\
\hline Folder name containing images: & \ImC-agr\AGR\ImageProcessing\P05122201\P0512220103\} $\\
{\hline}$
\end{tabular}

DMR calibration expiration date: $9 / 8 / 06$

Calibrated pixels/micron: 2.8260

Stage micrometer calibration expiration date: $2 / 17 / 07$

Measured value for $500 \mu \mathrm{m}$ in stage micrometer image $(\mu \mathrm{m}): 500.4$

\begin{tabular}{|c|c|c|c|}
\hline \multicolumn{4}{|c|}{ Polish-down distance $\mathrm{n}, \mathrm{m}(\mu \mathrm{m})$} \\
\hline 2,2 & 2,8 & 8,2 & 8,8 \\
\hline \hline 387 & 400 & 368 & 375 \\
\hline
\end{tabular}

\begin{tabular}{|c|c|c|c|c|}
\hline \multicolumn{5}{|c|}{ Approximate layer width in polish plane $(\mu \mathrm{m})$} \\
\hline Kernel radius & Buffer & IPyC & SiC & OPyC \\
\hline \hline 178 & 111 & 39 & 36 & 41 \\
\hline
\end{tabular}
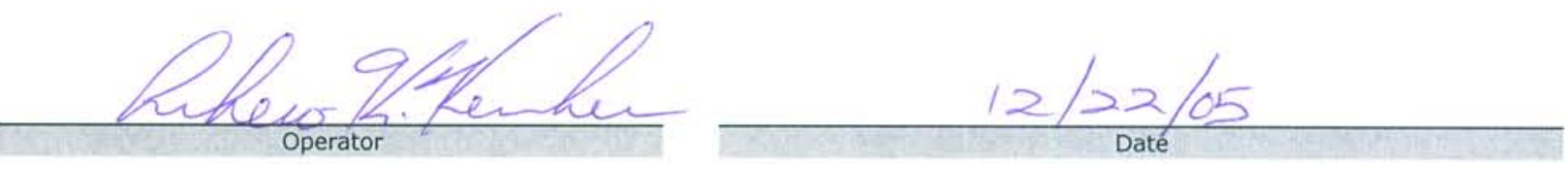
Data Report Form DRF-11A: Measurement of Buffer Layer Thickness

Procedure: AGR-CHAR-DAM-11 Rev, 1

Operator: Andrew K. Kercher

Folder name containing images: |Imc-agr|AGR|ImageProcessing|Completed_Layers\P05122201| Sample ID: LEU01-45T-B01

Sample Description: TRISO on BWXT composite 69302

Folder name containing processed data: \Imc-agr|AGR\ImageProcessing|Completed_Layers\P05122201_output

Number of buffer layers analyzed: $\quad 147$

Mean of the average buffer thickness of each particle (um):

Standard deviation in the average buffer thickness of each particle $(\mu \mathrm{m})$ :

Distribution of the average buffer layer thickness (top binned)

\begin{tabular}{|c|c|}
\hline Buffer Thickness $(\mu \mathrm{m})$ & Frequency \\
\hline \hline$<55$ & 0 \\
\hline 60 & 0 \\
\hline 65 & 0 \\
\hline 70 & 0 \\
\hline 75 & 0 \\
\hline 80 & 0 \\
\hline 85 & 0 \\
\hline 90 & 1 \\
\hline 95 & 4 \\
\hline 100 & 23 \\
\hline 105 & 35 \\
\hline 110 & 34 \\
\hline 115 & 23 \\
\hline 120 & 15 \\
\hline 125 & 9 \\
\hline$>125$ & 3 \\
\hline
\end{tabular}
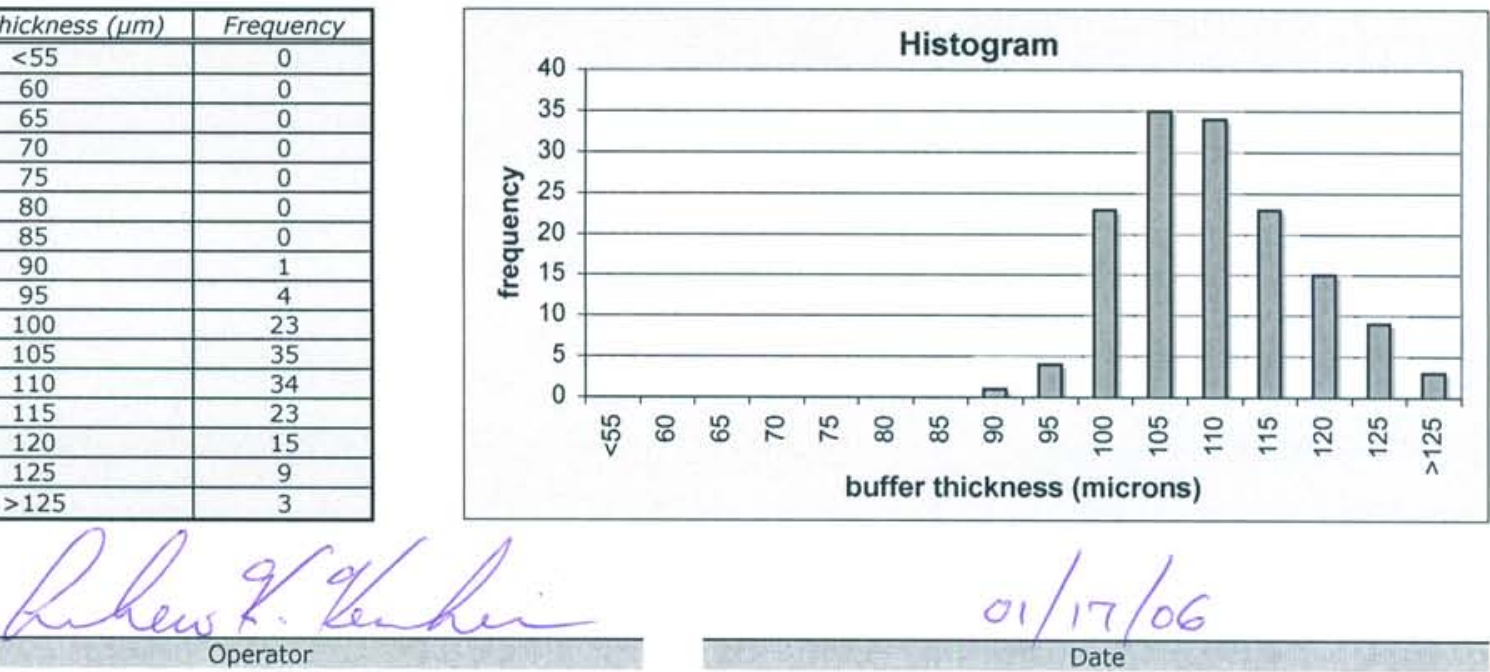
Data Report Form DRF-11B: Measurement of Inner Pyrocarbon Layer Thickness

\begin{tabular}{|c|c|}
\hline Procedure: & AGR-CHAR-DAM-11 Rev, 1 \\
\hline Operator: & Andrew K. Kercher \\
\hline Folder name containing images: & 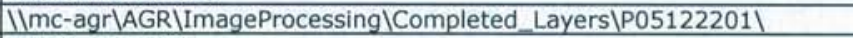 \\
\hline Sample ID: & LEU01-45T-B01 \\
\hline Sample Description: & TRISO on BWXT composite 69302 \\
\hline Folder name containing processed data: & IImc-agr|AGR\ImageProcessing|Completed_Layers\P05122201_output \\
\hline
\end{tabular}

\begin{tabular}{r|r|}
\hline Number of inner pyrocarbon layers analyzed: & 194 \\
\hline Mean of the average IPyC thickness of each particle $(\mu \mathrm{m}):$ & 40.1 \\
\hline Standard deviation in the average IPyC thickness of each particle $(\mu \mathrm{m}):$ & 2.0 \\
\hline
\end{tabular}

\section{Distribution of the average IPyC layer thickness (top binned)}

\begin{tabular}{|c|c|}
\hline IPyC Thickness $(\mu \mathrm{m})$ & Frequency \\
\hline \hline$<30$ & 0 \\
\hline 32 & 0 \\
\hline 34 & 0 \\
\hline 36 & 3 \\
\hline 38 & 23 \\
\hline 40 & 71 \\
\hline 42 & 65 \\
\hline 44 & 26 \\
\hline 46 & 4 \\
\hline 48 & 2 \\
\hline 50 & 0 \\
\hline 52 & 0 \\
\hline 54 & 0 \\
\hline 56 & 0 \\
\hline$>56$ & 0 \\
\hline
\end{tabular}
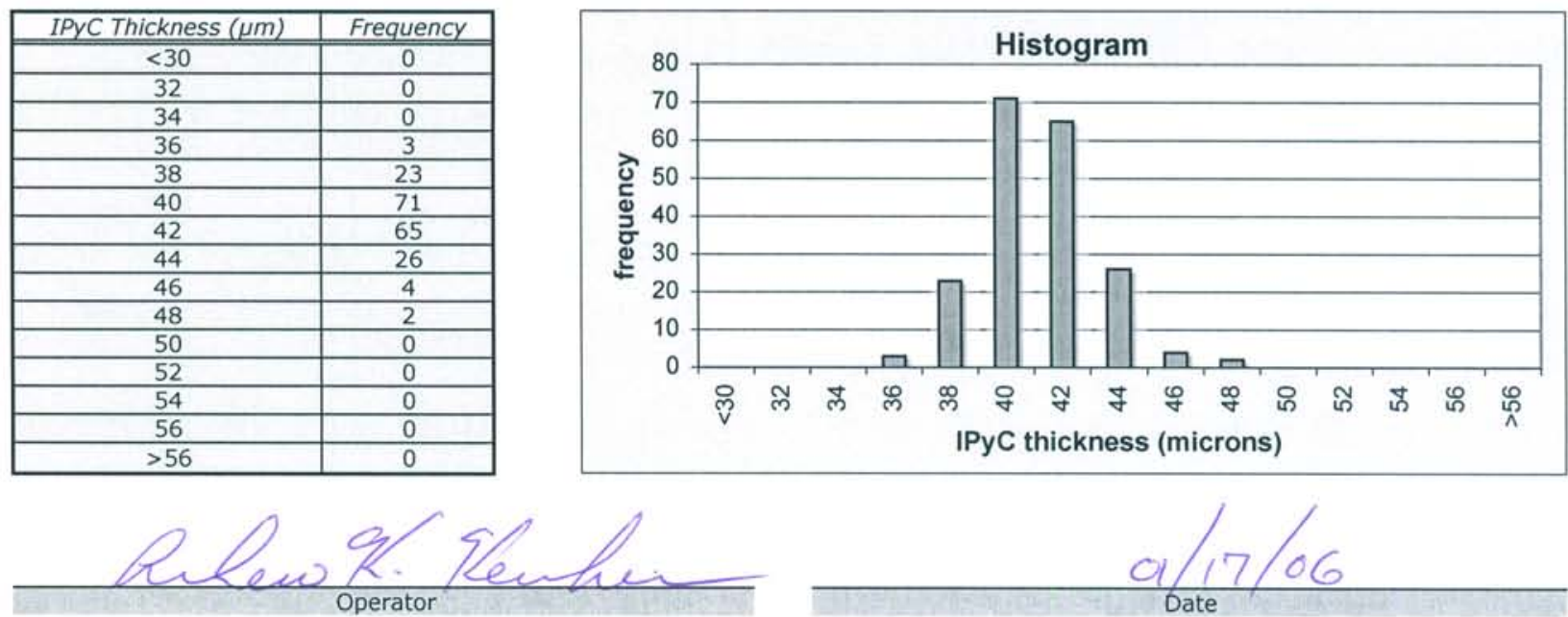
Data Report Form DRF-11C: Measurement of Silicon Carbide Layer Thickness

\begin{tabular}{|c|c|}
\hline Procedure: & AGR-CHAR-DAM-11 Rev. 1 \\
\hline Operator: & Andrew K. Kercher \\
\hline Folder name containing images: & IImc-agr|AGR\ImageProcessing|Completed Layers\P05122201】 \\
\hline Sample ID: & LEU01-45T-B01 \\
\hline Sample Description: & TRISO on BWXT composite 69302 \\
\hline Folder name containing processed data: & IImc-agr\AGR\ImageProcessing\Completed_Layers\P05122201_output \\
\hline
\end{tabular}

\begin{tabular}{|r|c|}
\hline Number of silicon carbide layers analyzed: & 194 \\
\hline Mean of the average SiC thickness of each particle $(\mu \mathrm{m}):$ & 35.8 \\
\hline Standard deviation in the average SiC thickness of each particle $(\mu \mathrm{m}):$ & 1.1 \\
\hline
\end{tabular}

\section{Distribution of the average SiC layer thickness (top binned)}

\begin{tabular}{|c|c|}
\hline SiC Thickness $(\mu \mathrm{m})$ & Frequency \\
\hline \hline 225 & 0 \\
\hline 26 & 0 \\
\hline 27 & 0 \\
\hline 28 & 0 \\
\hline 29 & 0 \\
\hline 30 & 0 \\
\hline 31 & 0 \\
\hline 32 & 0 \\
\hline 33 & 0 \\
\hline 34 & 15 \\
\hline 35 & 32 \\
\hline 36 & 63 \\
\hline 37 & 60 \\
\hline 38 & 20 \\
\hline 39 & 4 \\
\hline 40 & 0 \\
\hline$>40$ & 0 \\
\hline
\end{tabular}
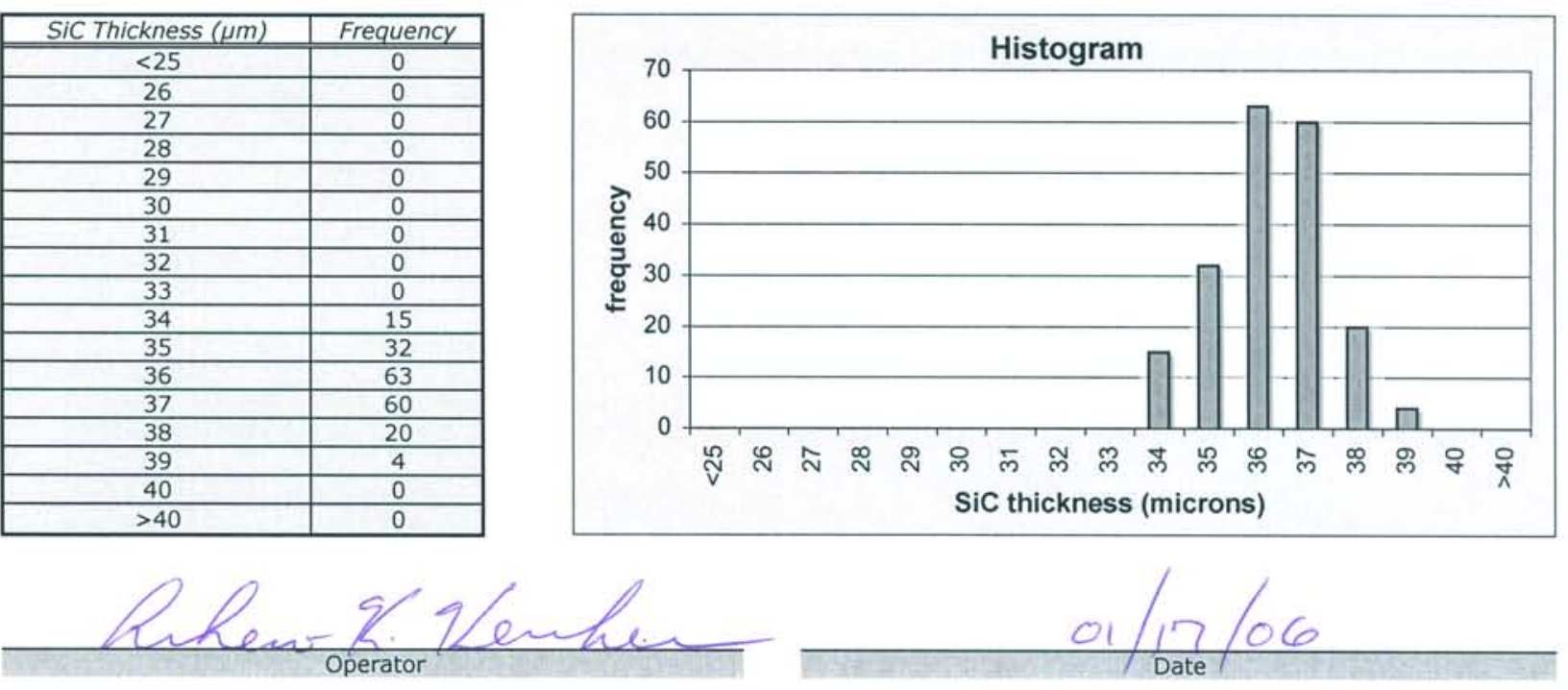
Data Report Form DRF-11D: Measurement of Outer Pyrocarbon Layer ThicknesS

\begin{tabular}{|c|c|}
\hline Procedure: & AGR-CHAR-DAM-11 Rev. 1 \\
\hline Operator: & Andrew K. Kercher \\
\hline Folder Name: & |Imc-agr|AGR\ImageProcessing|Completed_Layers\P05122201| \\
\hline Sample ID: & LEU01-45T-B01 \\
\hline Sample Description: & TRISO on BWXT composite 69302 \\
\hline Folder name containing processed data: & \Imc-agr|AGR\ImageProcessing|Completed_Layers\P05122201_output \\
\hline
\end{tabular}

Number of outer pyrocarbon layers analyzed:

Mean of the average OPyC thickness of each particle $(\mu \mathrm{m})$ : Standard deviation in the average OPyC thickness of each particle $(\mu \mathrm{m})$ :

Distribution of the average OPyC layer thickness (top binned)

\begin{tabular}{|c|c|}
\hline OPyC Thickness $(\mu \mathrm{m})$ & Frequency \\
\hline \hline 220 & 0 \\
\hline 22 & 0 \\
\hline 24 & 0 \\
\hline 26 & 0 \\
\hline 28 & 0 \\
\hline 30 & 0 \\
\hline 32 & 0 \\
\hline 34 & 0 \\
\hline 36 & 1 \\
\hline 38 & 25 \\
\hline 40 & 76 \\
\hline 42 & 62 \\
\hline 44 & 25 \\
\hline 46 & 5 \\
\hline 48 & 0 \\
\hline 50 & 0 \\
\hline$>50$ & 0 \\
\hline
\end{tabular}
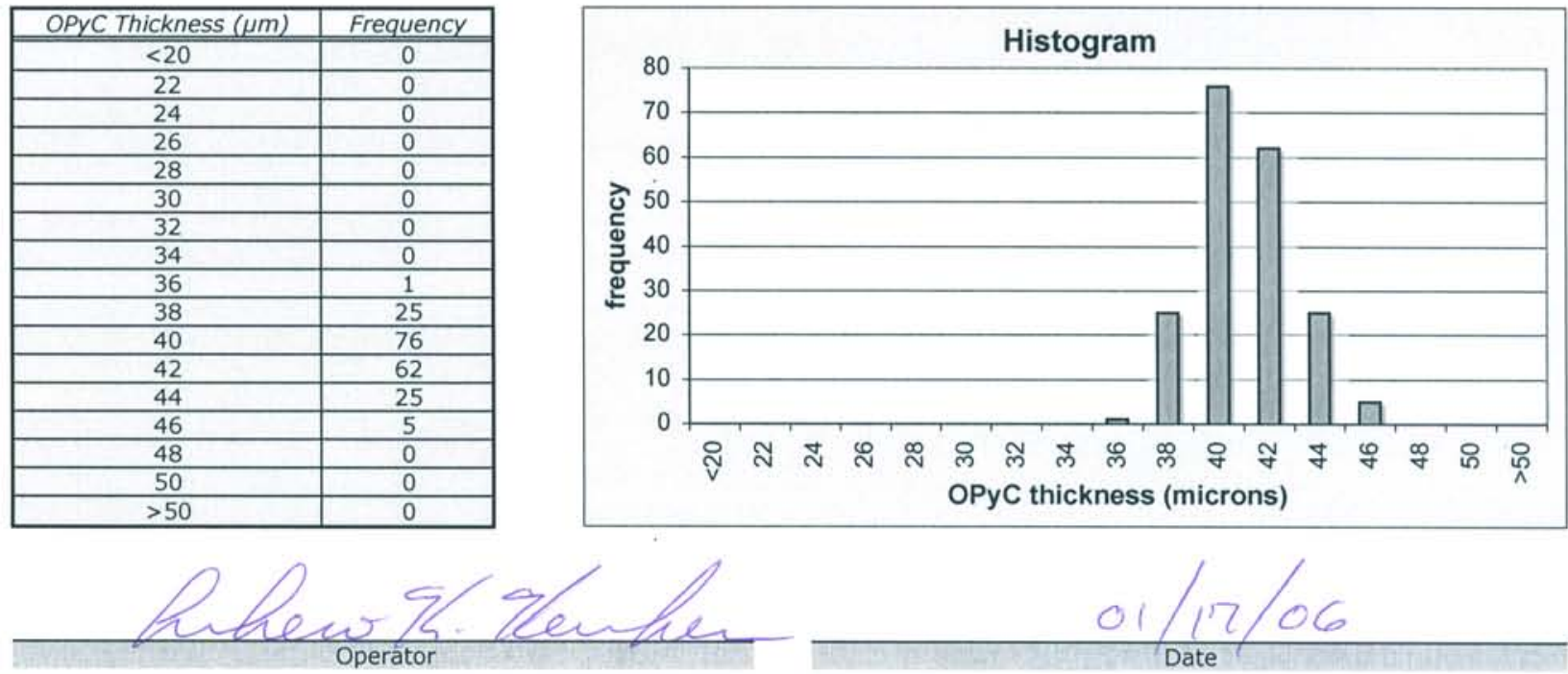
Data Report Form DRF-19: Counting of Particles with Missing OPyC Layer by Visual Inspection

\begin{tabular}{|r|l|}
\hline Procedure: & AGR-CHAR-DAM-19 Rev. 1 \\
\hline Operator: & John Hunn \\
\hline Sample ID: & LEU01-45T-C01 \\
\hline Sample Description: & TRISO on BWXT kernel composite 69302 \\
\hline Filename: & IImC-agr\AGR\MissingOPyCIX05122101_DRF19R1.xIs \\
\hline
\end{tabular}

\begin{tabular}{|r|l|}
\hline Mean average weight/particle $(\mathrm{g}):$ & $7.29 \mathrm{E}-04$ \\
\hline Uncertainty in average weight/particle $(\mathrm{g}):$ & $1.78 \mathrm{E}-06$ \\
\hline Weight of sample of particles $(\mathrm{g}):$ & 11.354 \\
\hline Approximate number of particles in sample: $:$ & 15574 \\
\hline Uncertainty in number of particles in sample: & 38 \\
\hline
\end{tabular}

Number of particles with missing OPyC layer: 0

\section{Comments on unusual visual characteristics of OPYC}

P0512210101: picture of slightly darker particle with some spots on surface.

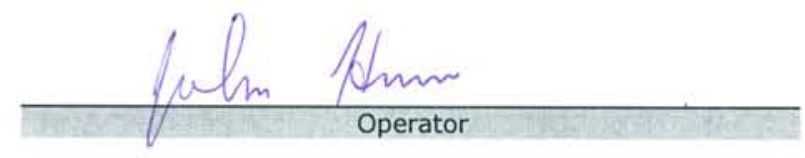

$$
12-21-05
$$

Date


Slightly darker particle with some spots on surface.

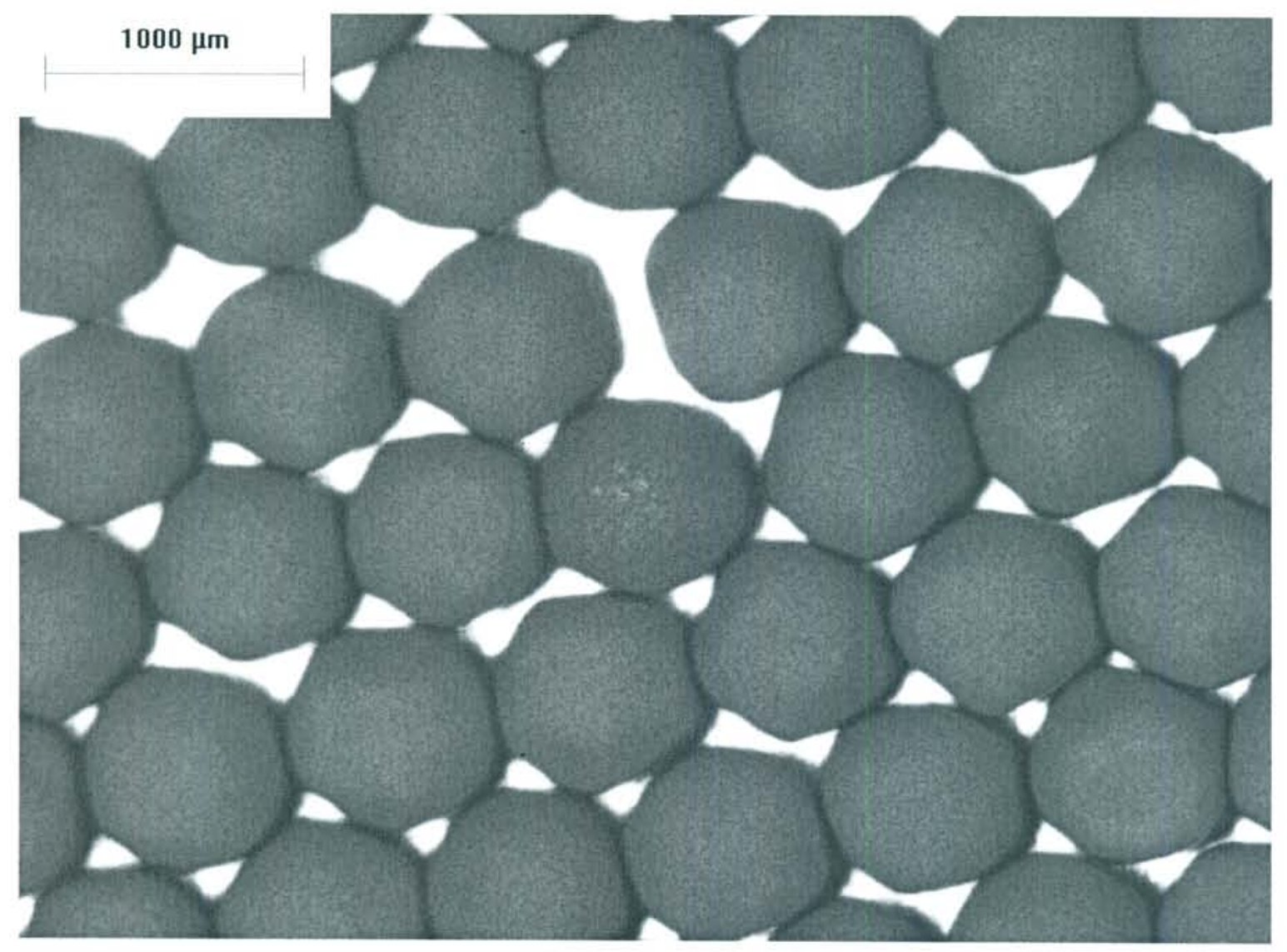




\section{For Information Only}

The information in the remainder of this section reports results of measurements not required by the fuel specification and is provided for information only. 
Data Report Form DRF-22: Estimation of Average Particle Weight

\begin{tabular}{|r|l|}
\hline Procedure: & AGR-CHAR-DAM-22 Rev. 1 \\
\hline Operator: & Dixie Barker \\
\hline Particle Lot ID: & LEU01-45T-D07 \\
\hline Particle Lot Description: & TRISO on BWXT kernel composite 69302 \\
\hline Filename: & IIImc-agr\AGR\ParticleWeight\W05122101_DRF22R1.xIs \\
\hline
\end{tabular}

\begin{tabular}{|r|c|c|c|c|c|}
\hline & Sample 1 & Sample 2 & Sample 3 & Sample 4 & Sample 5 \\
\hline Weight of particles (g): & $8.25 \mathrm{E}-02$ & $7.26 \mathrm{E}-02$ & $8.29 \mathrm{E}-02$ & $8.16 \mathrm{E}-02$ & $7.24 \mathrm{E}-02$ \\
\hline Number of particles: & 114 & 100 & 113 & 112 & 99 \\
\hline Average weight/particle (g): & $7.24 \mathrm{E}-04$ & $7.26 \mathrm{E}-04$ & $7.34 \mathrm{E}-04$ & $7.29 \mathrm{E}-04$ & $7.31 \mathrm{E}-04$ \\
\hline
\end{tabular}

Mean average weight/particle $(\mathrm{g}): 17.29 \mathrm{E}-04$

Uncertainty in mean average weight/particle $(g): 1.78 \mathrm{E}-06$
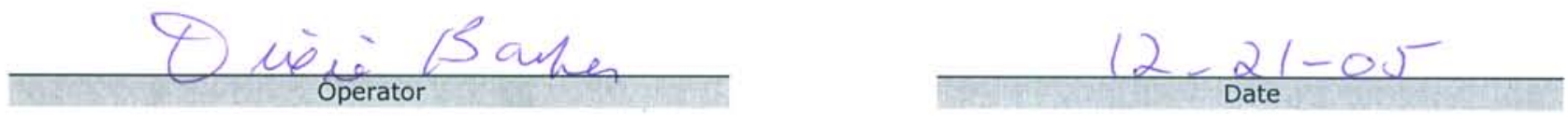
Data Report Form DRF-20: Counting of Particles with SiC Gold Spot Defects by Visual Inspection

\begin{tabular}{|r|l|}
\hline Procedure: & AGR-CHAR-DAM-20 Rev. 1 \\
\hline Operator: & John Hunn \\
\hline Sample ID: & LEU01-45T-C01 \\
\hline Sample Description: & TRISO on BWXT kernel composite 69302 \\
\hline Filename: & IImc-agr\AGR\GoldSpots\G05122101_DRF20R1.xIs \\
\hline
\end{tabular}

\begin{tabular}{|r|l|}
\hline Mean average weight/particle $(\mathrm{g}):$ & $7.29 \mathrm{E}-04$ \\
\hline Uncertainty in average weight/particle $(\mathrm{g}):$ & $1.78 \mathrm{E}-06$ \\
\hline Weight of sample of particles $(\mathrm{g}):$ & 11.354 \\
\hline Approximate number of particles in sample: & 15574 \\
\hline Uncertainty in number of particles in sample: & 38 \\
\hline
\end{tabular}

Number of particles with gold spot defects: 3

\section{Comments on unusual visual characteristics of SiC}

P0512210103: picture of 3 goldspots and one particle with white coating on surface. P0512210106: picture of average SiC surface appearance.

Goldspots were not obvious.

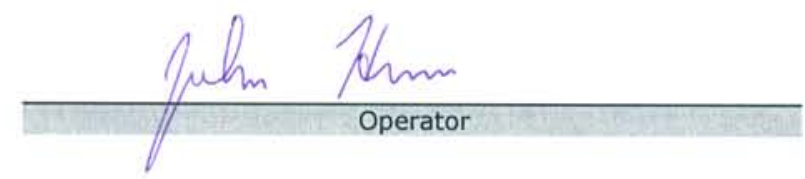

$$
\frac{12-21-05}{\text { Date }}
$$


3/15574 gold spots found. One particle had white coating on surface.

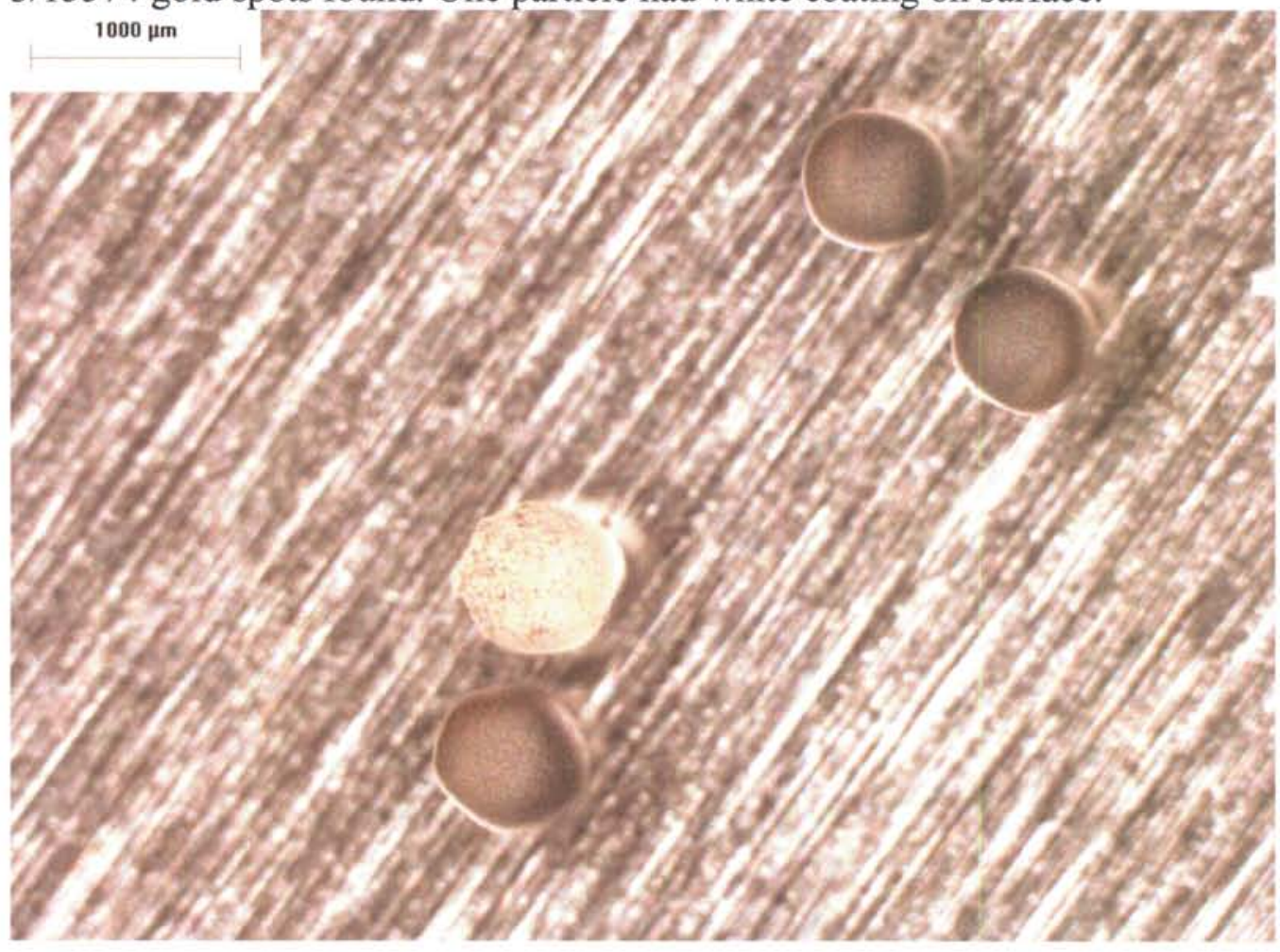

Average appearance of $\mathrm{SiC}$ after burnback for LEU01-45T

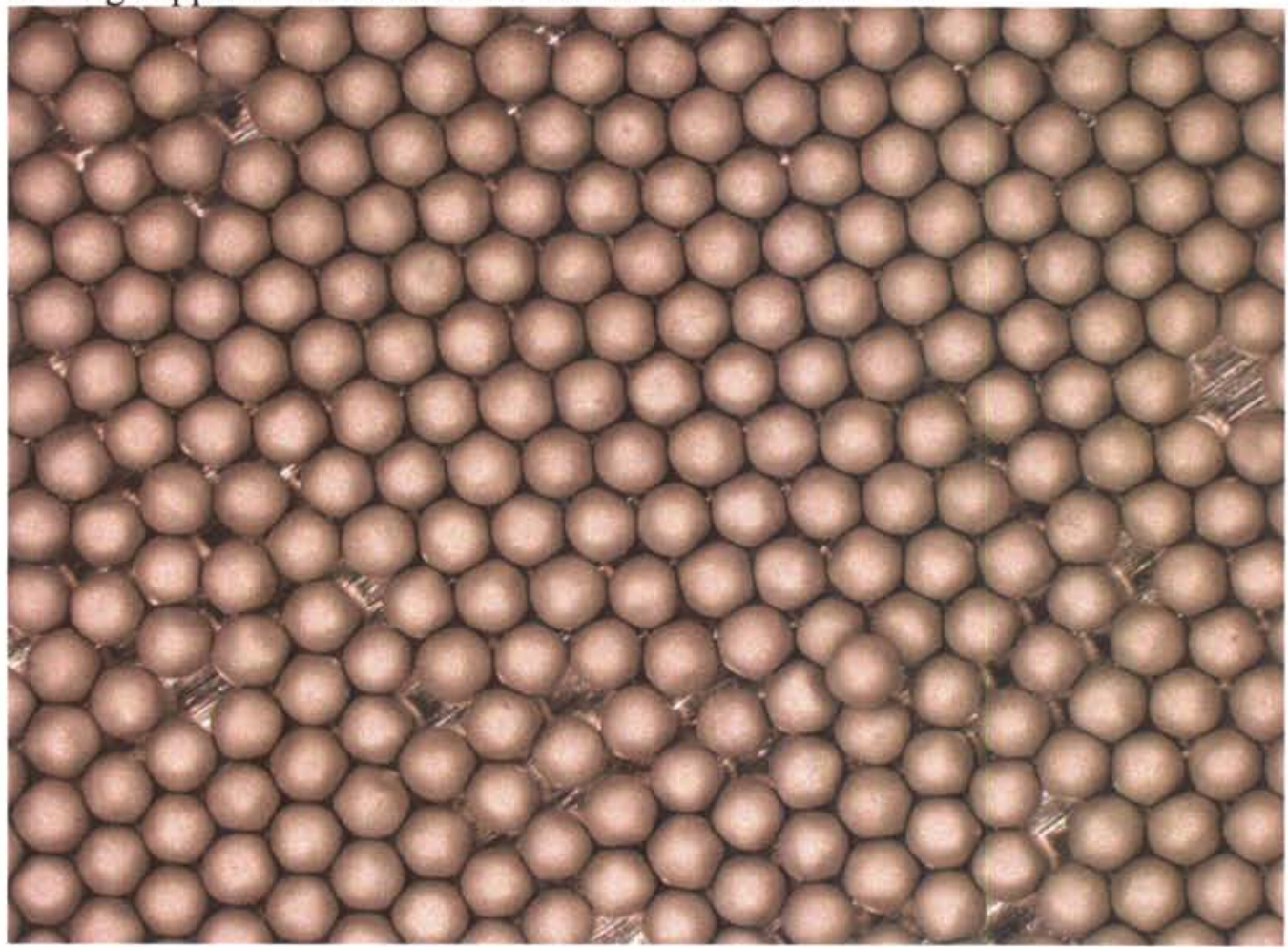




\section{Characterization of TRISO-coated particle composite}

This section contains data on the TRISO-coated particle composite, LEU01-46T. The data was obtained according to product inspection plan AGR-CHAR-PIP-04R2.

Note that some of the carbon deposited for the buffer layer reacted with the kernel to form a uranium carbide layer between the kernel and the buffer. The uranium carbide layer was not included in the measurement of the buffer thickness. The thickness of this carbide layer varied, but was typically around $6 \mu \mathrm{m}$ thick and effectively increased the kernel radius by that amount.

The following pages shows the inspection report forms (IRF-04A and IRF-04B) for the LEU0146T composite. Following IRF-04A and IRF-04B are the individual data report forms for the measurements that were performed. Additional data at the end of this section is provided for information only. This composite was determined to satisfy the specifications in section 5.3 of EDF 4380, Rev. 6. 
ORNL/TM-2006/019, Rev. 1

Inspection Report Form IRF-04A: Coated Particle Composites

Procedure: $\mid$ AGR-CHAR-PIP-04 Rev. 2

Coated particle composite ID: LEU01-46T

Coated particle composite description: Baseline Composite: TRISO on BWXT kernel composite 69302

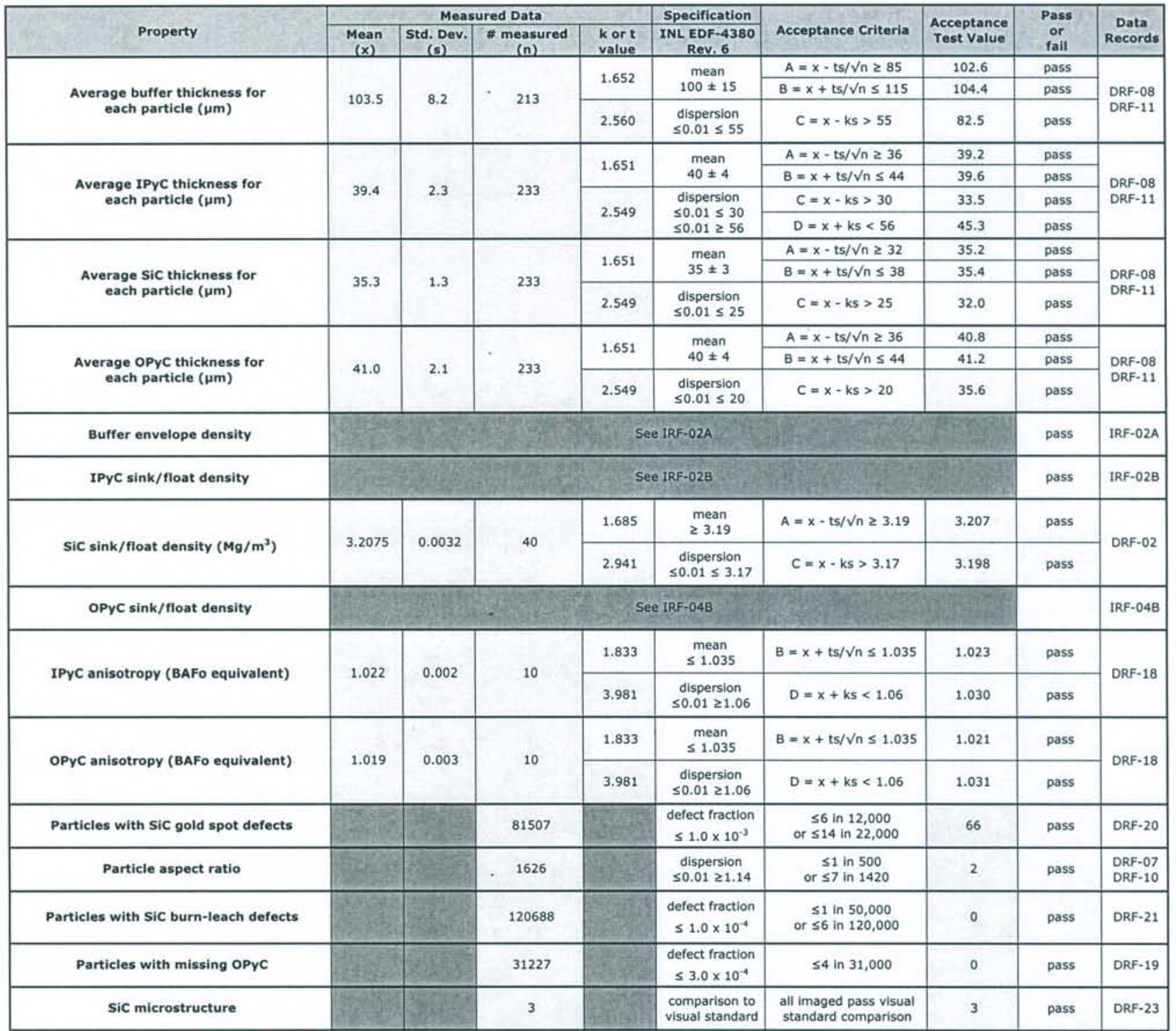


ORNL/TM-2006/019, Rev. 1

Inspection Report Form IRF-04B: Outer Pyrocarbon Density

\begin{tabular}{|c|c|c|c|c|c|c|c|c|c|}
\hline Procedure: & \multicolumn{9}{|c|}{ AGR-CHAR-PIP-04 Rev. 2.} \\
\hline Coated particle composite ID: & \multicolumn{9}{|c|}{ LEU01-46T } \\
\hline Coated particle composite description: & \multicolumn{9}{|c|}{ Baseline Composite: TRISO on BWXT kernel composite 69302} \\
\hline Batch 1 ID: & \multicolumn{9}{|c|}{ LEU01-21T } \\
\hline Batch 2 ID: & \multicolumn{9}{|c|}{ LEU01-35T } \\
\hline Batch 2 description: & \multicolumn{9}{|c|}{ Baseline TRISO on BWXT kernel composite 69302} \\
\hline Batch 3 ID: & \multicolumn{9}{|c|}{ LEU01-27T } \\
\hline Batch 3 description: & \multicolumn{9}{|c|}{ Baseline TRISO on BWXT kernel composite 69302} \\
\hline Batch 4 description: & \multicolumn{9}{|c|}{ Baseline TRISO on BWXT kernel composite 69302} \\
\hline \multirow[b]{2}{*}{ Property } & \multicolumn{4}{|c|}{ Measured Data } & Specification & \multirow[b]{2}{*}{ Acceptance Criteria } & \multirow{2}{*}{$\begin{array}{l}\text { Acceptance } \\
\text { Test Value }\end{array}$} & \multirow{2}{*}{$\begin{array}{c}\text { Pass } \\
\text { or } \\
\text { fail } \\
\end{array}$} & \multirow{2}{*}{$\begin{array}{l}\text { Data } \\
\text { Records }\end{array}$} \\
\hline & $\begin{array}{c}\text { Mean } \\
(x)\end{array}$ & $\begin{array}{c}\text { Std, Dev. } \\
(\mathrm{s})\end{array}$ & $\begin{array}{l}\text { \# measured } \\
(n)\end{array}$ & $\begin{array}{l}k \text { or } t \\
\text { value }\end{array}$ & $\begin{array}{c}\text { INL EDF- } 4380 \\
\text { Rev. } 6\end{array}$ & & & & \\
\hline \multirow{3}{*}{ Batch 1: OPyC sink/float density $\left(\mathrm{Mg} / \mathrm{m}^{3}\right)$} & \multirow{3}{*}{1.9121} & \multirow{3}{*}{0.0129} & \multirow{3}{*}{43} & \multirow{2}{*}{1.682} & \multirow{2}{*}{$\begin{array}{c}\text { mean } \\
1.90 \pm 0.05\end{array}$} & $A=x-t s / \sqrt{n} \geq 1.85$ & 1.909 & pass & \multirow{3}{*}{ DRF-03 } \\
\hline & & & & & & $B=x+t s / V n \leq 1.95$ & 1.915 & pass & \\
\hline & & & & 2.905 & $\begin{array}{c}\text { dispersion } \\
\leq 0.01 \leq 1.80 \\
\leq 0.01 \geq 2.00\end{array}$ & $C=x-k s>1.80$ & 1.875 & pass & \\
\hline \multirow{4}{*}{ Batch 2: OPyC sink/float density $\left(\mathrm{Mg} / \mathrm{m}^{3}\right)$} & \multirow{4}{*}{1.9029} & \multirow{4}{*}{0.0056} & \multirow{4}{*}{52} & 1.675 & mean & $A=x-t s / \sqrt{n} \geq 1.85$ & 1.902 & pass & \\
\hline & & & & & $1.90 \pm 0.05$ & $B=x+t s / \sqrt{ } n \leq 1.95$ & 1.904 & pass & 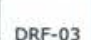 \\
\hline & & & & 2.842 & $\begin{array}{c}\text { dispersion } \\
\leq 0.01 \leq 1.80\end{array}$ & $C=x-k s>1.80$ & 1.887 & pass & Ukr $=03$ \\
\hline & & & & & $\leq 0.01 \geq 2.00$ & $D=x+k s<2.00$ & 1.919 & pass & \\
\hline & & & & 1680 & mean & $A=x-t s / \sqrt{ } n \geq 1.85$ & 1.902 & pass & \\
\hline & 1.9034 & 0.0054 & 45 & 2.000 & $1.90 \pm 0.05$ & $B=x+t s / \sqrt{ } n \leq 1.95$ & 1.905 & pass & DRF $=03$ \\
\hline Baten s: Opyc sink/noat density (mg/m? & & & & 2.897 & $\begin{array}{c}\text { dispersion } \\
\leq 0.01 \leq 1.80\end{array}$ & $C=x-k s>1.80$ & 1.888 & pass & Der $=03$ \\
\hline & & & & & $\leq 0.01 \geq 2.00$ & $D=x+k s<2.00$ & 1.919 & pass & \\
\hline & & & & 1.684 & mean & $A=x-t s / \sqrt{ } n \geq 1.85$ & 1.909 & pass & \\
\hline Batch 4: OPyC sink/float density $\left(\mathrm{Ma} / \mathrm{m}^{3}\right)$ & 1.9112 & 0.0076 & 41 & & $1.90 \pm 0.05$ & $B=x+t s / V n \leq 1.95$ & 1.913 & pass & DRF-03 \\
\hline 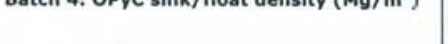 & & & 72 & 2.922 & $\begin{array}{c}\text { dispersion } \\
\leq 0.01 \leq 1.80\end{array}$ & $C=x-k s>1.80$ & 1.889 & pass & \\
\hline & & & & & $\leq 0.01 \geq 2.00$ & $D=x+k s<2.00$ & 1.933 & pass & \\
\hline
\end{tabular}
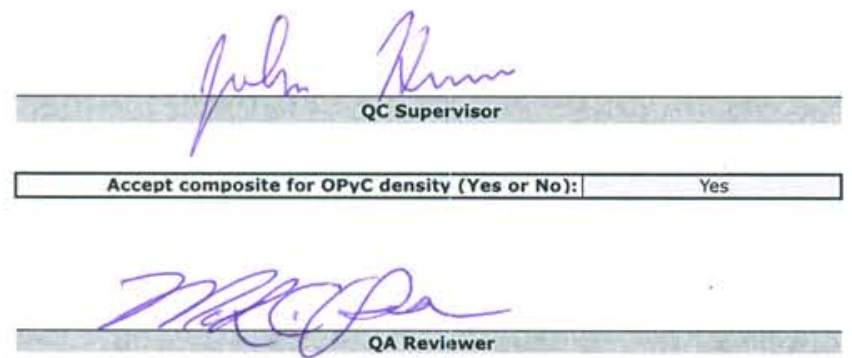

$3-21-06$

Date

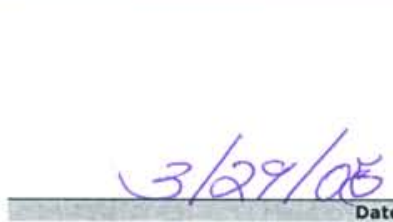


ORNL/TM-2006/019, Rev. 1

Data Report Form DRF-08: Imaging of Coated Particle Cross-sections Using an Optical Microscope System

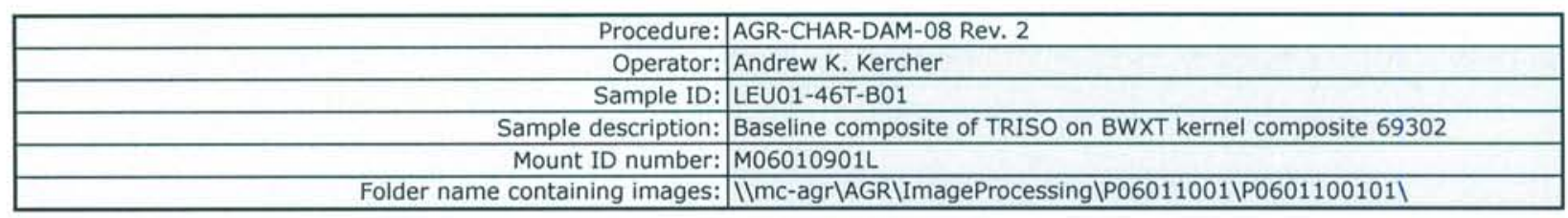

DMR calibration expiration date: $9 / 8 / 06$

Calibrated pixels/micron: 2.8260

Stage micrometer calibration expiration date: 2/17/07

Measured value for $500 \mu \mathrm{m}$ in stage micrometer image $(\mu \mathrm{m}): 500.7077$

\begin{tabular}{|c|c|c|c|}
\hline \multicolumn{4}{|c|}{ Polish-down distance $n, m(\mu \mathrm{m})$} \\
\hline 2,2 & 2,8 & 8,2 & 8,8 \\
\hline \hline 384 & 374 & 369 & 363 \\
\hline
\end{tabular}

\begin{tabular}{|c|c|c|c|c|}
\hline \multicolumn{5}{|c|}{ Approximate layer width in polish plane $(\mu \mathrm{m})$} \\
\hline Kernel radius & Buffer & IPyC & SiC & OPyC \\
\hline \hline 173 & 103 & 40 & 38 & 42 \\
\hline
\end{tabular}
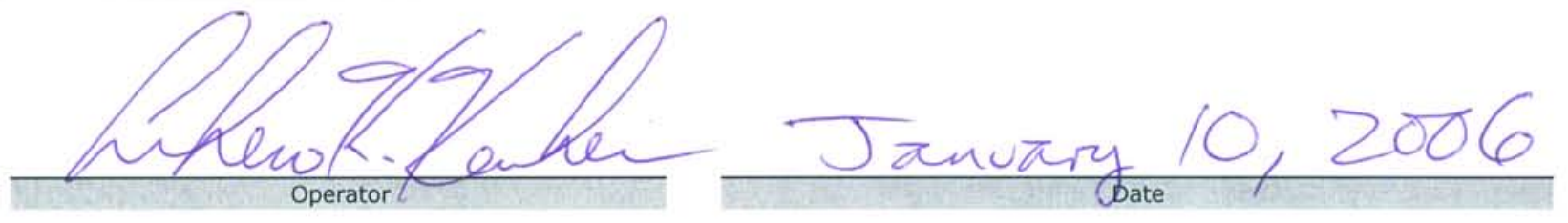
ORNL/TM-2006/019, Rev. 1

Data Report Form DRF-08: Imaging of Coated Particle Cross-sections Using an Optical Microscope System

\begin{tabular}{|r|l|}
\hline Procedure: & AGR-CHAR-DAM-08 Rev. 2 \\
\hline Operator: & Andrew K. Kercher \\
\hline Sample ID: & LEU01-46T-B01 \\
\hline Sample description: & Baseline composite of TRISO on BWXT kernel composite 69302 \\
\hline Mount ID number: & M06010902L \\
\hline Folder name containing images: & IImC-agr|AGR\ImageProcessing\P06011001\P0601100102\} $\\
{\hline}$
\end{tabular}

DMR calibration expiration date: $9 / 8 / 06$ Calibrated pixels/micron: 2.8260

Stage micrometer calibration expiration date: 2/17/07

Measured value for $500 \mu \mathrm{m}$ in stage micrometer image $(\mu \mathrm{m}): 500.7077$

\begin{tabular}{|c|c|c|c|c|c|c|c|}
\hline \multicolumn{3}{|c|}{ Polish-down distance $\mathrm{n}, \mathrm{m}(\mu \mathrm{m})$} \\
\hline 2,2 & 2,8 & 8,2 & 8,8 \\
\hline 373 & 378 & 353 & 354 \\
\cline { 3 - 7 }
\end{tabular}


ORNL/TM-2006/019, Rev. 1

Data Report Form DRF-08: Imaging of Coated Particle Cross-sections Using an Optical Microscope System

\begin{tabular}{|r|l|}
\hline Procedure: & AGR-CHAR-DAM-08 Rev. 2 \\
\hline Operator: & Andrew K. Kercher \\
\hline Sample ID: & LEU01-46T-B01 \\
\hline Sample description: & Baseline composite of TRISO on BWXT kernel composite 69302 \\
\hline Mount ID number: & M06010903L \\
\hline Folder name containing images: & \IMc-agr\AGR\ImageProcessing\P06011001\P0601100103\} $\\
{\hline}$
\end{tabular}

\begin{tabular}{|r|r|}
\hline DMR calibration expiration date: & $9 / 8 / 06$ \\
\hline Calibrated pixels/micron: & 2.8260 \\
\hline Stage micrometer calibration expiration date: & $2 / 17 / 07$ \\
\hline Measured value for $500 \mu \mathrm{m}$ in stage micrometer image $(\mu \mathrm{m}):$ & 500.7077 \\
\hline
\end{tabular}

\begin{tabular}{|c|c|c|c|}
\hline \multicolumn{4}{|c|}{ Polish-down distance $\mathrm{n}, \mathrm{m}(\mu \mathrm{m})$} \\
\hline 2,2 & 2,8 & 8,2 & 8,8 \\
\hline \hline 369 & 362 & 384 & 374 \\
\hline
\end{tabular}

\begin{tabular}{|c|c|c|c|c|}
\hline \multicolumn{5}{|c|}{ Approximate layer width in polish plane $(\mu \mathrm{m})$} \\
\hline Kernel radius & Buffer & IPyC & SiC & OPyC \\
\hline \hline 174 & 106 & 38 & 36 & 45 \\
\hline
\end{tabular}

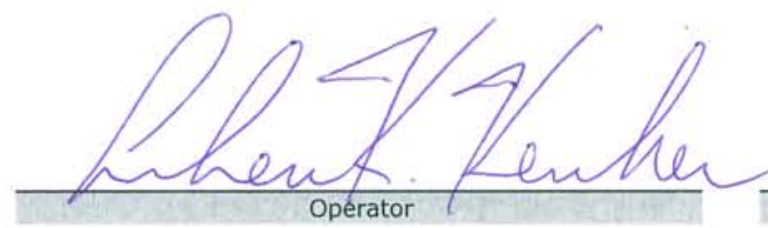
174 


\begin{tabular}{|c|c|c|}
\hline Procedure: & \multicolumn{2}{|l|}{ AGR-CHAR-DAM-11 Rev. 1} \\
\hline Operator: & \multicolumn{2}{|c|}{ Andrew K. Kercher } \\
\hline Folder name containing images: & \multicolumn{2}{|c|}{ 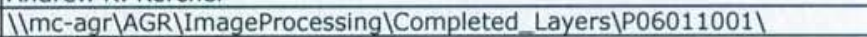 } \\
\hline Sample ID: & \multicolumn{2}{|c|}{ LEU01-46T-B01 } \\
\hline Sample Description: & \multicolumn{2}{|c|}{ Baseline Composite of TRISO on BWXT kernel composite 69302} \\
\hline Folder name containing processed data: & \multicolumn{2}{|c|}{ IImc-agr|AGR\ImageProcessing|Completed Layers\P06011001_output। } \\
\hline & Number of buffer layers analyzed: & 213 \\
\hline Mean of the & average buffer thickness of each particle $(\mu \mathrm{m})$ : & 103.5 \\
\hline Standard deviation in the & average buffer thickness of each particle $(\mu \mathrm{m})$ : & 8.2 \\
\hline
\end{tabular}

\section{Distribution of the average buffer layer thickness (top binned)}

\begin{tabular}{|c|c|}
\hline Buffer Thickness $(\mu \mathrm{m})$ & Frequency \\
\hline \hline$<55$ & 0 \\
\hline 60 & 0 \\
\hline 65 & 0 \\
\hline 70 & 0 \\
\hline 75 & 0 \\
\hline 80 & 0 \\
\hline 85 & 2 \\
\hline 90 & 10 \\
\hline 95 & 21 \\
\hline 100 & 34 \\
\hline 105 & 64 \\
\hline 110 & 42 \\
\hline 115 & 23 \\
\hline 120 & 10 \\
\hline 125 & 6 \\
\hline$>125$ & 1 \\
\hline
\end{tabular}
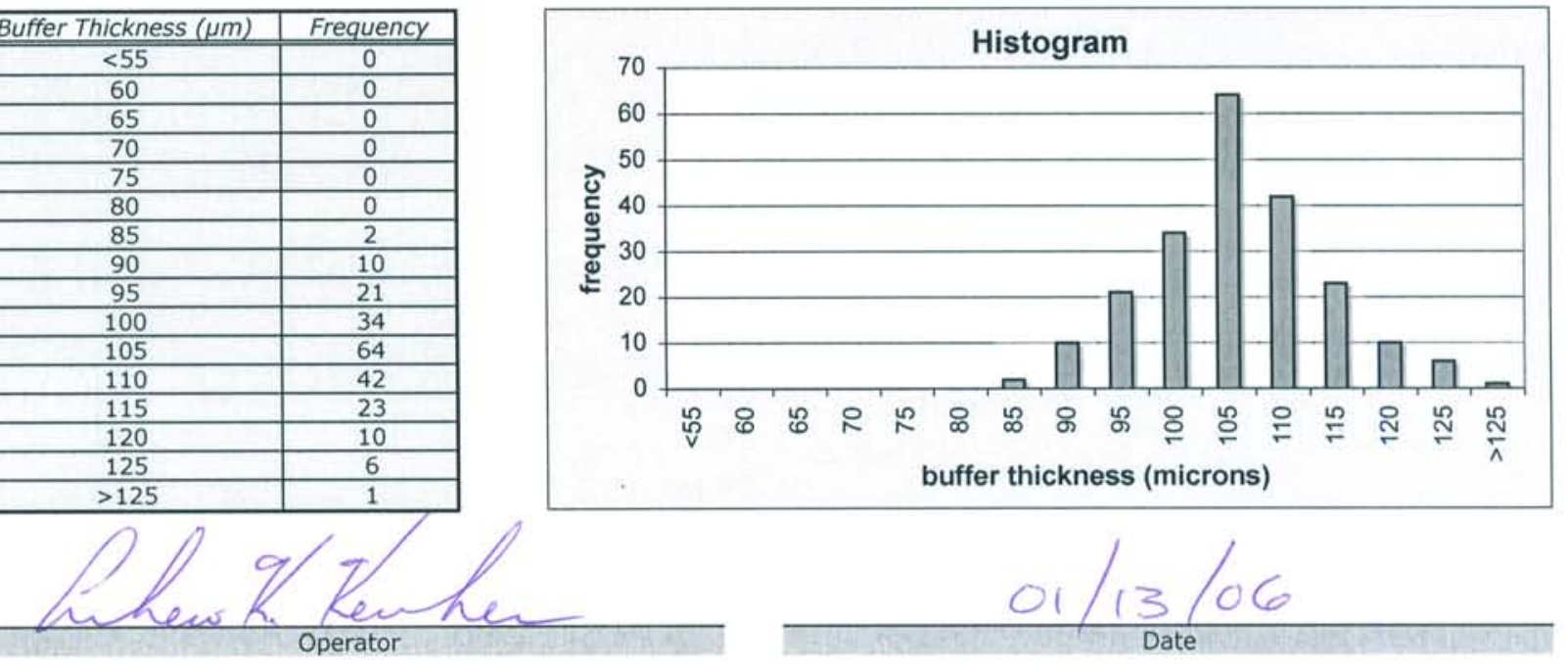
Data Report Form DRF-11B: Measurement of Inner Pyrocarbon Layer Thickness

Procedure: AGR-CHAR-DAM-11 Rev. 1

Operator: Andrew K. Kercher

Folder name containing images: IImc-agr|AGR\ImageProcessing|Completed_Layers\P06011001| Sample ID: LEU01-46T-B01

Sample Description: Baseline Composite of TRISO on BWXT kernel composite 69302

Folder name containing processed data: \Imc-agr|AGR\ImageProcessing|Completed_Layers\P06011001_output|

Number of inner pyrocarbon layers analyzed: $\quad 233$

Mean of the average IPyC thickness of each particle $(\mu \mathrm{m})$ :

Mean of the average IPyC thickness of each particle $(\mu \mathrm{m})$ :

2.3

Distribution of the average IPyC layer thickness (top binned)

\begin{tabular}{|c|c|}
\hline IPyC Thickness $(\mu \mathrm{m})$ & Frequency \\
\hline \hline$<30$ & 0 \\
\hline 32 & 0 \\
\hline 34 & 1 \\
\hline 36 & 9 \\
\hline 38 & 61 \\
\hline 40 & 84 \\
\hline 42 & 51 \\
\hline 44 & 17 \\
\hline 46 & 8 \\
\hline 48 & 1 \\
\hline 50 & 1 \\
\hline 52 & 0 \\
\hline 54 & 0 \\
\hline 56 & 0 \\
\hline$>56$ & 0 \\
\hline
\end{tabular}
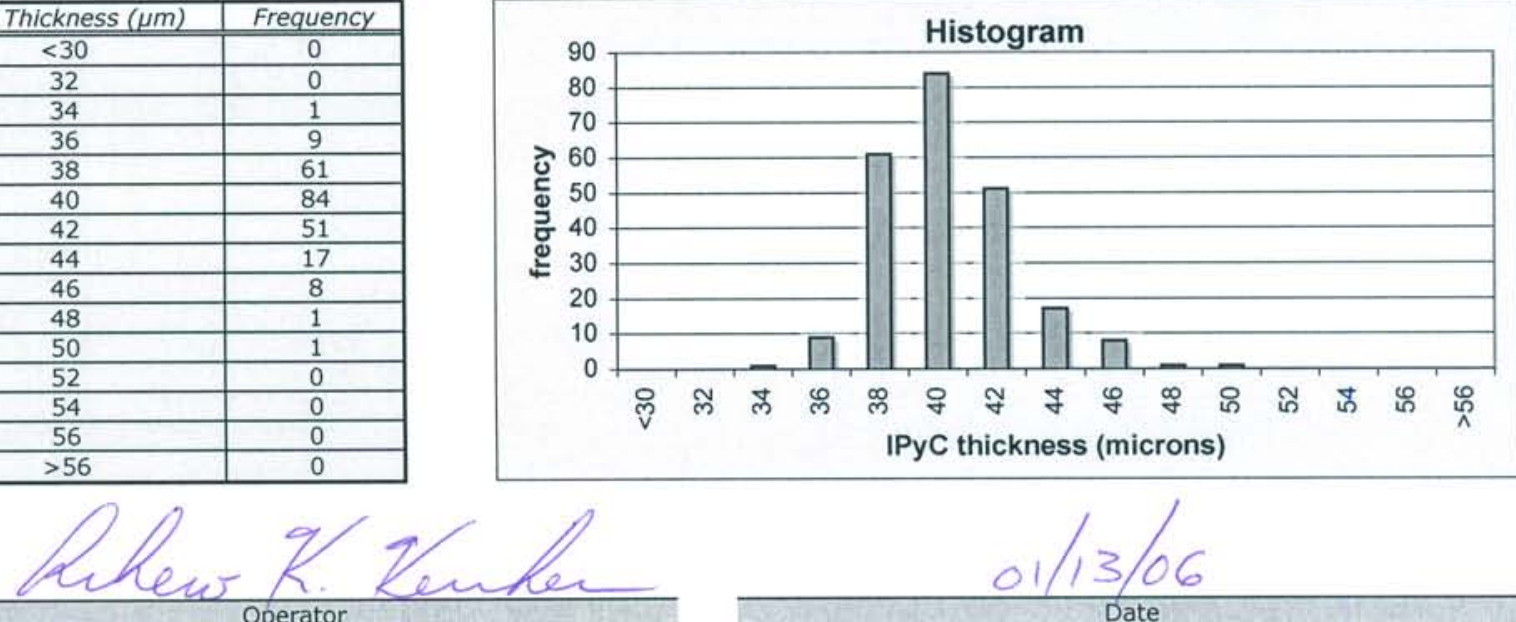

$01 / 3 / 06$ 
Procedure: AGR-CHAR-DAM-11 Rev. 1

Operator: Andrew K. Kercher

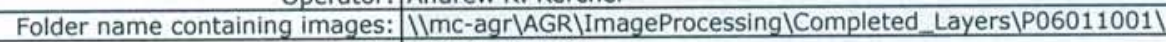
Sample ID: LEU01-46T-B01

Sample Description: Baseline Composite of TRISO on BWXT kernel composite 69302

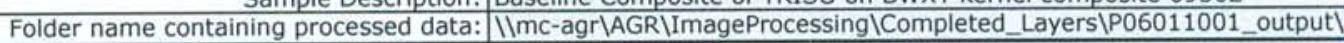

Number of silicon carbide layers analyzed:

Mean of the average SiC thickness of each particle $(\mu \mathrm{m})$ :

233

Standard deviation in the average SiC thickness of each particle $(\mu \mathrm{m})$ :

Distribution of the average SiC layer thickness (top binned)

\begin{tabular}{|c|c|}
\hline SiC Thickness $(\mu \mathrm{m})$ & Frequency \\
\hline \hline$<25$ & 0 \\
\hline 26 & 0 \\
\hline 27 & 0 \\
\hline 28 & 0 \\
\hline 29 & 0 \\
\hline 30 & 0 \\
\hline 31 & 0 \\
\hline 32 & 0 \\
\hline 33 & 7 \\
\hline 34 & 31 \\
\hline 35 & 59 \\
\hline 36 & 65 \\
\hline 37 & 41 \\
\hline 38 & 25 \\
\hline 39 & 4 \\
\hline 40 & 1 \\
\hline$>40$ & 0 \\
\hline & \\
\hline
\end{tabular}
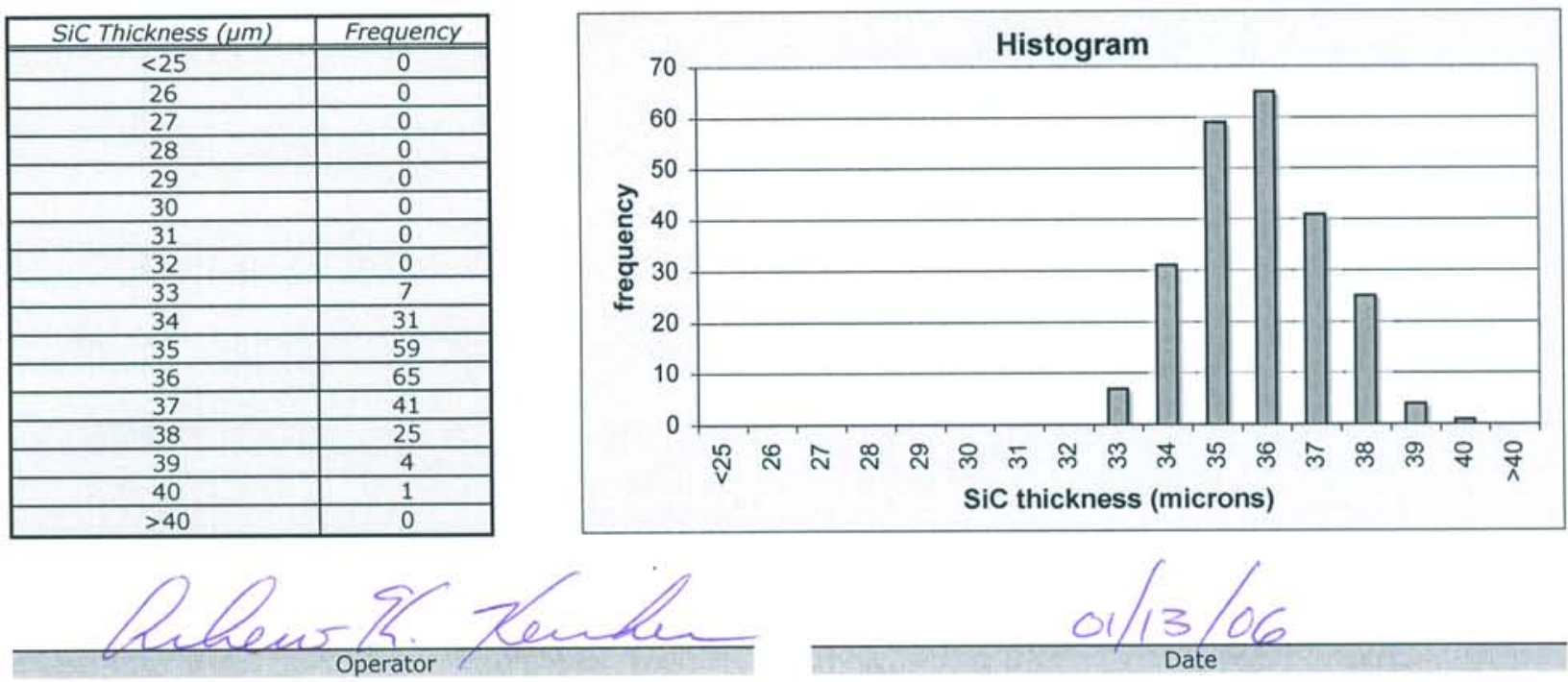


\begin{tabular}{|c|c|}
\hline Procedure: & AGR-CHAR-DAM-11 Rev. 1 \\
\hline Operator: & Andrew K. Kercher \\
\hline Folder Name: & |IImc-agr|AGR|ImageProcessing|Completed_Layers|P06011001| \\
\hline Sample ID: & LEU01-46T-B01 \\
\hline Sample Description: & Baseline Composite of TRISO on BWXT kernel composite 69302 \\
\hline Folder name containing processed data: & 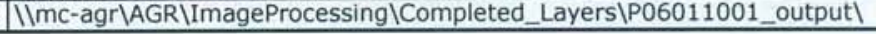 \\
\hline
\end{tabular}

\begin{tabular}{|r|c|}
\hline Number of outer pyrocarbon layers analyzed: & 233 \\
\hline Mean of the average OPyC thickness of each particle $(\mu \mathrm{m}):$ & 41.0 \\
\hline Standard deviation in the average OPyC thickness of each particle $(\mu \mathrm{m}):$ & 2.1 \\
\hline
\end{tabular}

\section{Distribution of the average OPyC layer thickness (top binned)}

\begin{tabular}{|c|c|}
\hline OPyC Thickness $(\mu \mathrm{m})$ & Frequency \\
\hline \hline 220 & 0 \\
\hline 22 & 0 \\
\hline 24 & 0 \\
\hline 26 & 0 \\
\hline 28 & 0 \\
\hline 30 & 0 \\
\hline 32 & 0 \\
\hline 34 & 0 \\
\hline 36 & 0 \\
\hline 38 & 19 \\
\hline 40 & 51 \\
\hline 42 & 95 \\
\hline 44 & 48 \\
\hline 46 & 17 \\
\hline 48 & 2 \\
\hline 50 & 1 \\
\hline$>50$ & 0 \\
\hline
\end{tabular}
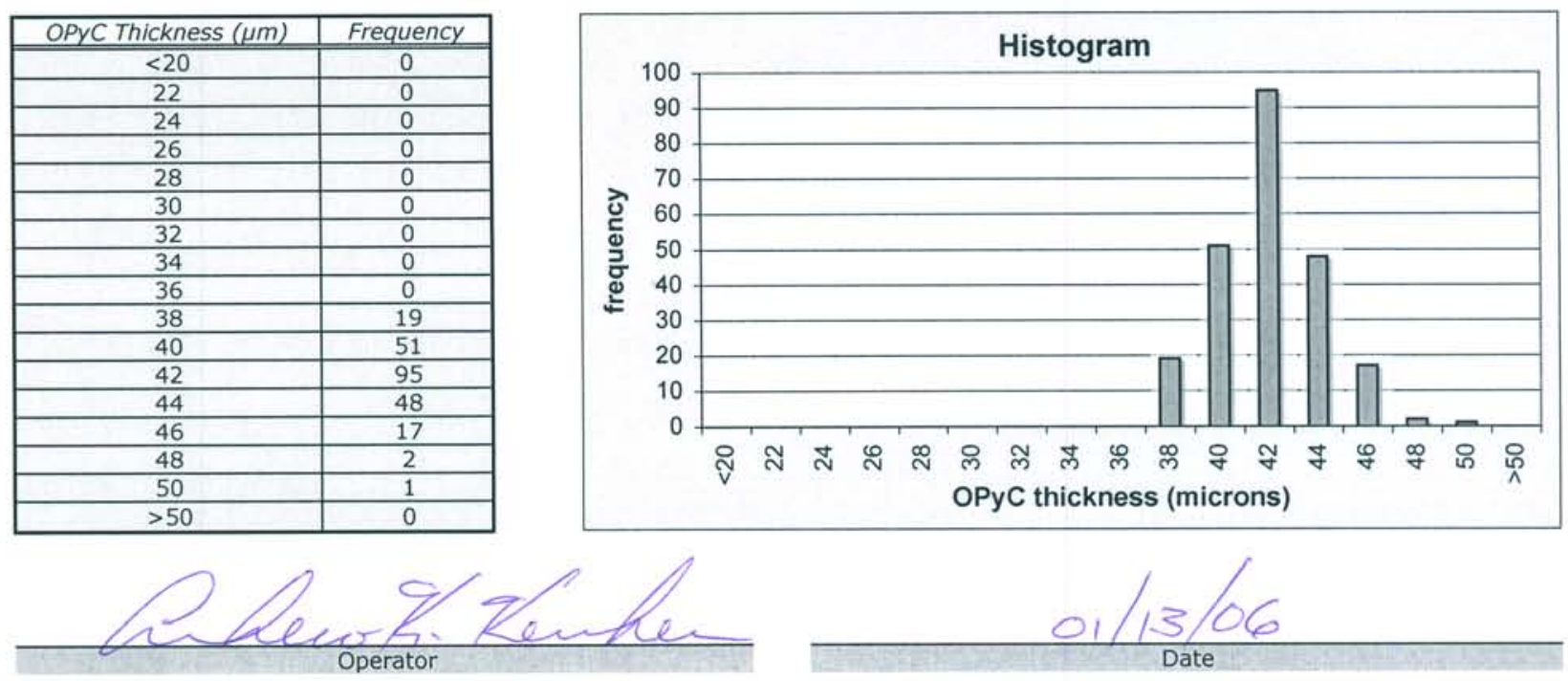
ORNL/TM-2006/019, Rev. 1

Data Report Form DRF-02: Measurement of SiC Density using a Density Gradient Column

\begin{tabular}{|r|l|}
\hline Procedure: & AGR-CHAR-DAM-02 Rev. 3 \\
\hline Operator: & Dixie Barker \\
\hline Filename: & IImc-agr\AGRIDensityColumn\D06010901_DRF02R3.xIs \\
\hline Sample ID: & LEU01-46T-E01 \\
\hline Sample description: & Baseline Composite of TRISO on BWXT kernel composite 69302 \\
\hline Float expiration date: & $07 / 2007$ \\
\hline Gauge expiration date: & $01 / 2007$ \\
\hline Bath temperature: & $23.0^{\circ} \mathrm{C}$ \\
\hline
\end{tabular}

\begin{tabular}{|c|c|c|c|}
\hline \multicolumn{4}{|c|}{ Calibrated Floats } \\
\hline Density & $\begin{array}{c}\text { Top of } \\
\text { Float }\end{array}$ & $\begin{array}{c}\text { Bottom of } \\
\text { Float }\end{array}$ & $\begin{array}{c}\text { Center of } \\
\text { Mass }\end{array}$ \\
\hline 3.150 & 153.01 & 173.52 & 166.68 \\
\hline 3.170 & 280.32 & 304.28 & 296.29 \\
\hline 3.190 & 385.96 & 408.33 & 400.87 \\
\hline 3.210 & 508.72 & 532.91 & 524.85 \\
\hline
\end{tabular}

\begin{tabular}{|c|c|c|c|}
\hline \multicolumn{4}{|c|}{ Linear Fit } \\
\hline slope & StDev & intercept & StDev \\
\hline \hline $1.70 \mathrm{E}-04$ & $3.14 \mathrm{E}-06$ & $3.12 \mathrm{E}+00$ & $1.09 \mathrm{E}-03$ \\
\hline
\end{tabular}

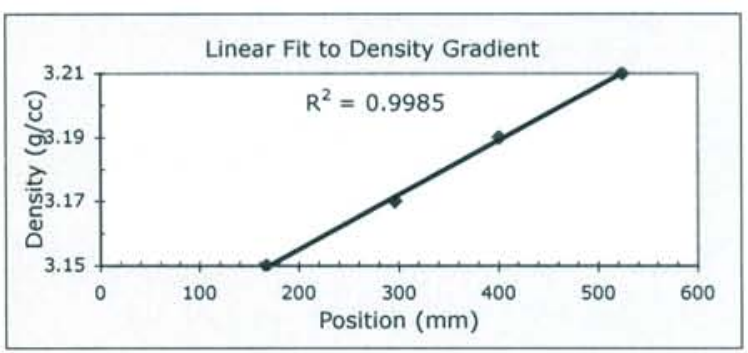

\begin{tabular}{|c|c|c|c|c|c|c|c|c|}
\hline \multicolumn{9}{|c|}{ Sample Density } \\
\hline $\begin{array}{l}\text { Fragment } \\
\text { Number }\end{array}$ & $\begin{array}{l}\text { Fragment } \\
\text { Position }\end{array}$ & $\begin{array}{c}\text { Calculated } \\
\text { Density }\end{array}$ & $\begin{array}{l}\text { Fragment } \\
\text { Number }\end{array}$ & $\begin{array}{c}\text { Fragment } \\
\text { Position }\end{array}$ & $\begin{array}{l}\text { Calculated } \\
\text { Density }\end{array}$ & $\begin{array}{l}\text { Fragment } \\
\text { Number }\end{array}$ & $\begin{array}{c}\text { Fragment } \\
\text { Position }\end{array}$ & $\begin{array}{l}\text { Calculated } \\
\text { Density }\end{array}$ \\
\hline 1 & 475.63 & 3.2018 & 26 & 511.65 & 3.2079 & 51 & & \\
\hline 2 & 481.23 & 3.2027 & 27 & 515.56 & 3.2086 & 52 & & \\
\hline 3 & 482.49 & 3.2030 & 28 & 516.88 & 3.2088 & 53 & & \\
\hline 4 & 487.46 & 3.2038 & 29 & 517.99 & 3.2090 & 54 & & \\
\hline 5 & 488.57 & 3.2040 & 30 & 519.89 & 3.2093 & 55 & & \\
\hline 6 & 489.19 & 3.2041 & 31 & 522.05 & 3.2097 & 56 & & \\
\hline 7 & 490.04 & 3.2042 & 32 & 525.39 & 3.2102 & 57 & & \\
\hline 8 & 491.48 & 3.2045 & 33 & 528.52 & 3.2108 & 58 & & \\
\hline 9 & 494.40 & 3.2050 & 34 & 534.03 & 3.2117 & 59 & & \\
\hline 10 & 495.87 & 3.2052 & 35 & 536.34 & 3.2121 & 60 & & \\
\hline 11 & 496.75 & 3.2054 & 36 & 542.73 & 3.2132 & 61 & & \\
\hline 12 & 496.72 & 3.2054 & 37 & 547.81 & 3.2140 & 62 & & \\
\hline 13 & 497.30 & 3.2055 & 38 & 548.98 & 3.2142 & 63 & & \\
\hline 14 & 497.82 & 3.2056 & 39 & 542.93 & 3.2132 & 64 & & \\
\hline 15 & 499.70 & 3.2059 & 40 & 532.80 & 3.2115 & 65 & & \\
\hline 16 & 501.31 & 3.2061 & 41 & & & 66 & & \\
\hline 17 & 501.31 & 3.2061 & 42 & & & 67 & & \\
\hline 18 & 503.80 & 3.2066 & 43 & & & 68 & & \\
\hline 19 & 504.50 & 3.2067 & 44 & & & 69 & & \\
\hline 20 & 504.50 & 3.2067 & 45 & & & 70 & & \\
\hline 21 & 506.53 & 3.2070 & 46 & & & 71 & & \\
\hline 22 & 507.10 & 3.2071 & 47 & & & 72 & & \\
\hline 23 & 508.81 & 3.2074 & 48 & & & 73 & & \\
\hline 24 & 510.12 & 3.2076 & 49 & & & 74 & & \\
\hline 25 & 511.03 & 3.2078 & 50 & & & 75 & & \\
\hline \multicolumn{4}{|c|}{ Average density of SiC fragments: } & \multicolumn{5}{|c|}{3.2075} \\
\hline \multicolumn{4}{|c|}{ Standard deviation in density of SiC fragments: } & \multicolumn{5}{|c|}{0.0032} \\
\hline \multicolumn{4}{|c|}{ Uncertainty in calculated density of SiC fragments: } & \multicolumn{5}{|c|}{0.0020} \\
\hline
\end{tabular}
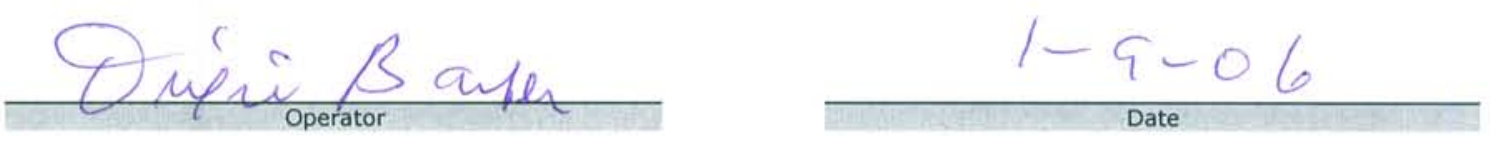


\begin{tabular}{|r|l|}
\hline Procedure: & AGR-CHAR-DAM-03 Rev. 2 \\
\hline Operator: & Dixie Barker \\
\hline Filename: & IImc-agr\AGRIDensityColumn\D05121201_DRF03R2.xIs \\
\hline Sample ID: & LEU01-35T-E01 \\
\hline Sample description: & OPyC fragments from TRISO on BWXT kernel composite 69302 \\
\hline Float expiration date: & $07 / 2007$ \\
\hline Gauge expiration date: & $01 / 2006$ \\
\hline Bath temperature: & $22.9^{\circ} \mathrm{C}$ \\
\hline
\end{tabular}

\begin{tabular}{|c|c|c|c|}
\hline \multicolumn{4}{|c|}{ Calibrated Floats } \\
\hline Density & $\begin{array}{l}\text { Top of } \\
\text { Float }\end{array}$ & $\begin{array}{l}\text { Bottom of } \\
\text { Float }\end{array}$ & $\begin{array}{c}\text { Center of } \\
\text { Mass }\end{array}$ \\
\hline 1.800 & 119.98 & 129.36 & 124.67 \\
\hline 1.850 & 213.68 & 222.19 & 217.94 \\
\hline 1.900 & 314.03 & 319.46 & 316.75 \\
\hline 1.950 & 416.29 & 424.36 & 420.33 \\
\hline 2.000 & 523.90 & 530.89 & 527.40 \\
\hline \multicolumn{4}{|c|}{ Linear Fit } \\
\hline slope & StDev & intercept & StDev \\
\hline $4.96 \mathrm{E}-04$ & $3.19 E-06$ & $1.74 E+00$ & 1.10E-03 \\
\hline
\end{tabular}

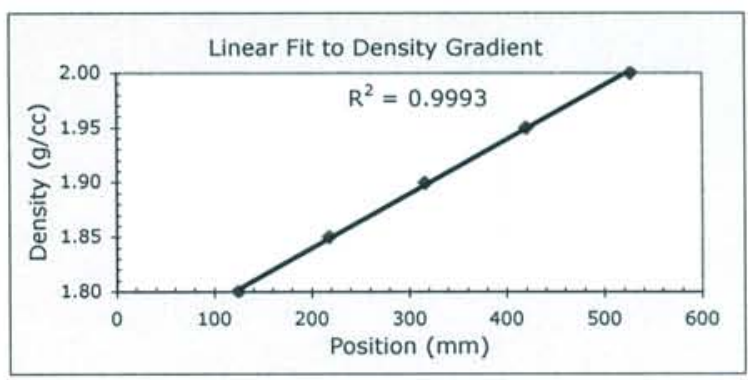

\begin{tabular}{|c|c|c|c|c|c|c|c|c|}
\hline \multicolumn{9}{|c|}{ Sample Density } \\
\hline $\begin{array}{l}\text { Fragment } \\
\text { Number }\end{array}$ & $\begin{array}{l}\text { Fragment } \\
\text { Position } \\
\end{array}$ & $\begin{array}{c}\text { Calculated } \\
\text { Density }\end{array}$ & $\begin{array}{c}\text { Fragment } \\
\text { Number }\end{array}$ & $\begin{array}{l}\text { Fragment } \\
\text { Position }\end{array}$ & $\begin{array}{c}\text { Calculated } \\
\text { Density }\end{array}$ & $\begin{array}{c}\text { Fragment } \\
\text { Number }\end{array}$ & $\begin{array}{l}\text { Fragment } \\
\text { Position }\end{array}$ & $\begin{array}{c}\text { Calculated } \\
\text { Density }\end{array}$ \\
\hline 1 & 300.95 & 1.8899 & 26 & 328.21 & 1.9034 & 51 & 350.91 & 1.9146 \\
\hline 2 & 301.89 & 1.8903 & 27 & 328.21 & 1.9034 & 52 & 351.43 & 1.9149 \\
\hline 3 & 308.92 & 1.8938 & 28 & 329.21 & 1.9039 & 53 & & \\
\hline 4 & 309.95 & 1.8943 & 29 & 329.21 & 1.9039 & 54 & & \\
\hline 5 & 310.76 & 1.8947 & 30 & 329.57 & 1.9040 & 55 & & \\
\hline 6 & 311.56 & 1.8951 & 31 & 330.26 & 1.9044 & 56 & & \\
\hline 7 & 312.08 & 1.8954 & 32 & 330.53 & 1.9045 & 57 & & \\
\hline 8 & 314.28 & 1.8965 & 33 & 330.96 & 1.9047 & 58 & & \\
\hline 9 & 314.67 & 1.8967 & 34 & 331.42 & 1.9050 & 59 & & \\
\hline 10 & 318.32 & 1.8985 & 35 & 332.02 & 1.9053 & 60 & & \\
\hline 11 & 317.98 & 1.8983 & 36 & 331.93 & 1.9052 & 61 & & \\
\hline 12 & 318.34 & 1.8985 & 37 & 332.31 & 1.9054 & 62 & & \\
\hline 13 & 326.12 & 1.9023 & 38 & 332.85 & 1.9057 & 63 & & \\
\hline 14 & 321.12 & 1.8999 & 39 & 334.27 & 1.9064 & 64 & & \\
\hline 15 & 322.49 & 1.9005 & 40 & 334.27 & 1.9064 & 65 & & \\
\hline 16 & 322.62 & 1.9006 & 41 & 335.56 & 1.9070 & 66 & & \\
\hline 17 & 322.82 & 1.9007 & 42 & 336.51 & 1.9075 & 67 & & \\
\hline 18 & 320.93 & 1.8998 & 43 & 337.51 & 1.9080 & 68 & & \\
\hline 19 & 321.46 & 1.9000 & 44 & 337.71 & 1.9081 & 69 & & \\
\hline 20 & 322.26 & 1.9004 & 45 & 339.41 & 1.9089 & 70 & & \\
\hline 21 & 323.96 & 1.9013 & 46 & 339.70 & 1.9091 & 71 & & \\
\hline 22 & 324.71 & 1.9016 & 47 & 340.21 & 1.9093 & 72 & & \\
\hline 23 & 325.52 & 1.9020 & 48 & 342.83 & 1.9106 & 73 & & \\
\hline 24 & 327.00 & 1.9028 & 49 & 344.33 & 1.9114 & 74 & & \\
\hline 25 & 327.67 & 1.9031 & 50 & 345.16 & 1.9118 & 75 & & \\
\hline \multicolumn{4}{|c|}{ Average density of PyC fragments: } & \multicolumn{5}{|c|}{1.9029} \\
\hline \multicolumn{4}{|c|}{ Standard deviation in density of PyC fragments: } & \multicolumn{5}{|c|}{0.0056} \\
\hline \multicolumn{4}{|c|}{ Uncertainty in calculated density of PyC fragments: } & \multicolumn{5}{|c|}{0.0016} \\
\hline
\end{tabular}
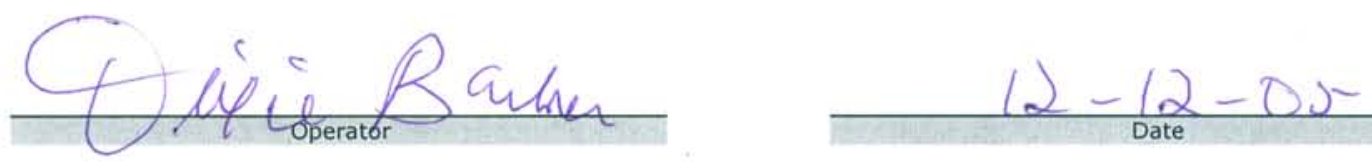
ORNL/TM-2006/019, Rev. 1

Data Report Form DRF-03: Measurement of PyC Density using a Density Gradient Column

\begin{tabular}{|r|l|}
\hline Procedure: & AGR-CHAR-DAM-03 Rev. 2 \\
\hline Operator: & Dixie Barker \\
\hline Filename: & IImC-agr\AGR\DensityColumn\D05122001_DRF03R2.xIs \\
\hline Sample ID: & LEU01-27T-E01 \\
\hline Sample description: & OPyC fragments from TRISO on BWXT kernel composite 69302 \\
\hline Float expiration date: & $07 / 2007$ \\
\hline Gauge expiration date: & $01 / 2006$ \\
\hline Bath temperature: & $23.7^{\circ} \mathrm{C}$ \\
\hline
\end{tabular}

\begin{tabular}{|c|c|c|c|}
\hline \multicolumn{4}{|c|}{ Calibrated Floats } \\
\hline Density & $\begin{array}{c}\text { Top of } \\
\text { Float }\end{array}$ & $\begin{array}{c}\text { Bottom of } \\
\text { Float }\end{array}$ & $\begin{array}{c}\text { Center of } \\
\text { Mass }\end{array}$ \\
\hline \hline 1.800 & 129.31 & 138.58 & 133.95 \\
\hline 1.850 & 224.09 & 232.28 & 228.19 \\
\hline 1.900 & 324.34 & 329.86 & 327.10 \\
\hline 1.950 & 426.34 & 433.52 & 429.93 \\
\hline 2.000 & 530.56 & 537.79 & 534.18 \\
\hline
\end{tabular}

\begin{tabular}{|c|c|c|c|}
\hline \multicolumn{4}{|c|}{ Linear Fit } \\
\hline slope & StDev & intercept & StDev \\
\hline \hline $4.99 \mathrm{E}-04$ & $3.38 \mathrm{E}-06$ & $1.74 \mathrm{E}+00$ & $1.12 \mathrm{E}-03$ \\
\hline
\end{tabular}

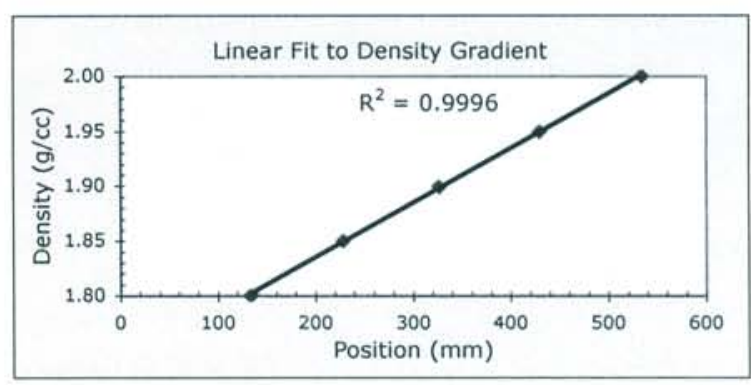

\begin{tabular}{|c|c|c|c|c|c|c|c|c|}
\hline \multicolumn{9}{|c|}{ Sample Density } \\
\hline $\begin{array}{l}\text { Fragment } \\
\text { Number }\end{array}$ & $\begin{array}{l}\text { Fragment } \\
\text { Position }\end{array}$ & $\begin{array}{c}\text { Calculated } \\
\text { Density }\end{array}$ & $\begin{array}{l}\text { Fragment } \\
\text { Number }\end{array}$ & $\begin{array}{l}\text { Fragment } \\
\text { Position }\end{array}$ & $\begin{array}{l}\text { Calculated } \\
\text { Density }\end{array}$ & $\begin{array}{l}\text { Fragment } \\
\text { Number }\end{array}$ & $\begin{array}{l}\text { Fragment } \\
\text { Position }\end{array}$ & $\begin{array}{l}\text { Calculated } \\
\text { Density }\end{array}$ \\
\hline 1 & 314.59 & 1.8920 & 26 & 339.98 & 1.9046 & 51 & & \\
\hline 2 & 316.85 & 1.8931 & 27 & 340.25 & 1.9048 & 52 & & \\
\hline 3 & 318.98 & 1.8942 & 28 & .341 .25 & 1.9053 & 53 & & \\
\hline 4 & 322.19 & 1.8958 & 29 & 341.67 & 1.9055 & 54 & & \\
\hline 5 & 323.42 & 1.8964 & 30 & 342.32 & 1.9058 & 55 & & \\
\hline 6 & 327.18 & 1.8983 & 31 & 342.92 & 1.9061 & 56 & & \\
\hline 7 & 326.91 & 1.8981 & 32 & 344.70 & 1.9070 & 57 & & \\
\hline 8 & 329.64 & 1.8995 & 33 & 345.80 & 1.9075 & 58 & & \\
\hline 9 & 328.07 & 1.8987 & 34 & 346.65 & 1.9080 & 59 & & \\
\hline 10 & 329.80 & 1.8996 & 35 & 346.65 & 1.9080 & 60 & & \\
\hline 11 & 330.67 & 1.9000 & 36 & 347.44 & 1.9084 & 61 & & \\
\hline 12 & 330.67 & 1.9000 & 37 & 349.45 & 1.9094 & 62 & & \\
\hline 13 & 331.97 & 1.9006 & 38 & 349.45 & 1.9094 & 63 & & \\
\hline 14 & 332.43 & 1.9009 & 39 & 350.04 & 1.9097 & 64 & & \\
\hline 15 & 333.11 & 1.9012 & 40 & 351.01 & 1.9101 & 65 & & \\
\hline 16 & 333.18 & 1.9013 & 41 & 351.01 & 1.9101 & 66 & & \\
\hline 17 & 333.19 & 1.9013 & 42 & 353.47 & 1.9114 & 67 & & \\
\hline 18 & 334.75 & 1.9020 & 43 & 355.22 & 1.9122 & 68 & & \\
\hline 19 & 335.35 & 1.9023 & 44 & 363.11 & 1.9162 & 69 & & \\
\hline 20 & 336.51 & 1.9029 & 45 & 322.11 & 1.8957 & 70 & & \\
\hline 21 & 337.27 & 1.9033 & 46 & & & 71 & & \\
\hline 22 & 337.66 & 1.9035 & 47 & & & 72 & & \\
\hline 23 & 338.08 & 1.9037 & 48 & & & 73 & & \\
\hline 24 & 338.56 & 1.9039 & 49 & & & 74 & & \\
\hline 25 & 339.98 & 1.9046 & 50 & & & 75 & & \\
\hline \multicolumn{4}{|c|}{ Average density of PyC fragments: } & \multicolumn{5}{|c|}{1.9034} \\
\hline \multirow{2}{*}{\multicolumn{4}{|c|}{$\begin{array}{r}\text { Standard deviation in density of PyC fragments: } \\
\text { Uncertainty in calculated density of PyC fragments: }\end{array}$}} & \multicolumn{5}{|c|}{0.0054} \\
\hline & & & & \multicolumn{5}{|c|}{0.0017} \\
\hline
\end{tabular}

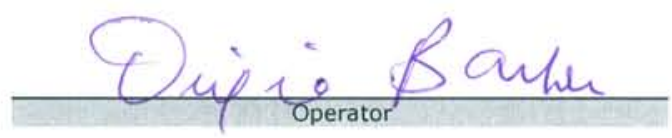

$\frac{12-20-05}{\text { Date }}$ 
ORNL/TM-2006/019, Rev. 1

Data Report Form DRF-03: Measurement of PyC Density using a Density Gradient Column

\begin{tabular}{|r|l|}
\hline Procedure: & AGR-CHAR-DAM-03 Rev. 2 \\
\hline Operator: & Dixie Barker \\
\hline Filename: & IImc-agr\AGRIDensityColumn\D05122101_DRF03R2.xIS \\
\hline Sample ID: & LEU01-45T-E01 \\
\hline Sample description: & OPyC fragments from TRISO on BWXT kernel composite 69302 \\
\hline Float expiration date: & $07 / 2007$ \\
\hline Gauge expiration date: & $01 / 2007$ \\
\hline Bath temperature: & $23.7^{\circ} \mathrm{C}$ \\
\hline
\end{tabular}

\begin{tabular}{|c|c|c|c|}
\hline \multicolumn{4}{|c|}{ Calibrated Floats } \\
\hline Density & $\begin{array}{c}\text { Top of } \\
\text { Float }\end{array}$ & $\begin{array}{c}\text { Bottom of } \\
\text { Float }\end{array}$ & $\begin{array}{c}\text { Center of } \\
\text { Mass }\end{array}$ \\
\hline \hline 1.800 & 142.66 & 151.71 & 147.19 \\
\hline 1.850 & 253.82 & 262.20 & 258.01 \\
\hline 1.900 & 365.64 & 371.28 & 368.46 \\
\hline 1.950 & 470.49 & 477.14 & 473.82 \\
\hline 2.000 & 567.86 & 575.43 & 571.65 \\
\hline
\end{tabular}

\begin{tabular}{|c|c|c|c|}
\hline \multicolumn{4}{|c|}{ Linear Fit } \\
\hline slope & StDev & intercept & StDev \\
\hline \hline $4.69 \mathrm{E}-04$ & $2.85 \mathrm{E}-06$ & $1.73 \mathrm{E}+00$ & $1.12 \mathrm{E}-03$ \\
\hline
\end{tabular}

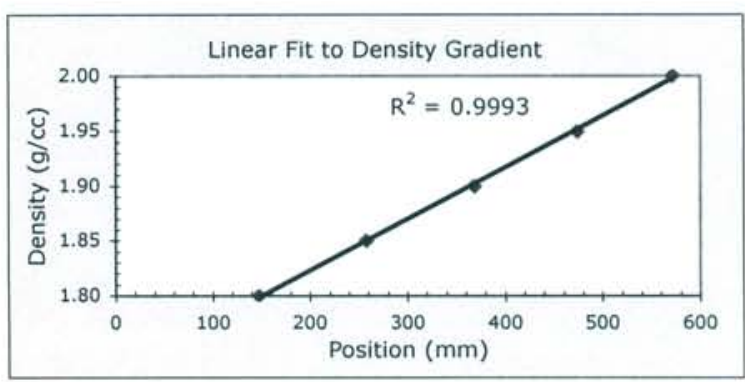

\begin{tabular}{|c|c|c|c|c|c|c|c|c|}
\hline \multicolumn{9}{|c|}{ Sample Density } \\
\hline $\begin{array}{l}\text { Fragment } \\
\text { Number }\end{array}$ & $\begin{array}{c}\text { Fragment } \\
\text { Position }\end{array}$ & $\begin{array}{c}\text { Calculated } \\
\text { Density }\end{array}$ & $\begin{array}{c}\text { Fragment } \\
\text { Number }\end{array}$ & $\begin{array}{c}\text { Fragment } \\
\text { Position }\end{array}$ & $\begin{array}{c}\text { Calculated } \\
\text { Density }\end{array}$ & $\begin{array}{c}\text { Fragment } \\
\text { Number }\end{array}$ & $\begin{array}{c}\text { Fragment } \\
\text { Position }\end{array}$ & $\begin{array}{c}\text { Calculated } \\
\text { Density } \\
\end{array}$ \\
\hline 1 & 343.23 & 1.8903 & 26 & 401.70 & 1.9178 & 51 & & \\
\hline 2 & 353.43 & 1.8951 & 27 & 402.90 & 1.9183 & 52 & & \\
\hline 3 & 355.52 & 1.8961 & 28 & 403.67 & 1.9187 & 53 & & \\
\hline 4 & 359.74 & 1.8981 & 29 & 407.15 & 1.9203 & 54 & & \\
\hline 5 & 367.37 & 1.9017 & 30 & 408.64 & 1.9210 & 55 & & \\
\hline 6 & 372.98 & 1.9043 & 31 & 409.85 & 1.9216 & 56 & & \\
\hline 7 & 377.40 & 1.9064 & 32 & 411.44 & 1.9223 & 57 & & \\
\hline 8 & 378.06 & 1.9067 & 33 & 416.75 & 1.9248 & 58 & & \\
\hline 9 & 378.08 & 1.9067 & 34 & 403.78 & 1.9188 & 59 & & \\
\hline 10 & 379.79 & 1.9075 & 35 & 396.95 & 1.9155 & 60 & & \\
\hline 11 & 380.98 & 1.9081 & 36 & 396.95 & 1.9155 & 61 & & \\
\hline 12 & 382.91 & 1.9090 & 37 & 395.84 & 1.9150 & 62 & & \\
\hline 13 & 383.64 & 1.9093 & 38 & 389.83 & 1.9122 & 63 & & \\
\hline 14 & 384.46 & 1.9097 & 39 & 385.32 & 1.9101 & 64 & & \\
\hline 15 & 385.69 & 1.9103 & 40 & 378.92 & 1.9071 & 65 & & \\
\hline 16 & 386.07 & 1.9104 & 41 & 377.31 & 1.9063 & 66 & & \\
\hline 17 & 388.17 & 1.9114 & 42 & & & 67 & & \\
\hline 18 & 388.88 & 1.9118 & 43 & & & 68 & & \\
\hline 19 & 389.34 & 1.9120 & 44 & & & 69 & & \\
\hline 20 & 392.45 & 1.9134 & 45 & & & 70 & & \\
\hline 21 & 393.74 & 1.9140 & 46 & & & 71 & & \\
\hline 22 & 394.90 & 1.9146 & 47 & & & 72 & & \\
\hline 23 & 396.03 & 1.9151 & 48 & & & 73 & & \\
\hline 24 & 397.30 & 1.9157 & 49 & & & 74 & & \\
\hline 25 & 398.29 & 1.9162 & 50 & & & 75 & & \\
\hline \multicolumn{4}{|c|}{$\begin{array}{r}\text { Average density of PyC fragments: } \\
\end{array}$} & \multicolumn{5}{|c|}{1.9112} \\
\hline \multicolumn{4}{|c|}{ Standard deviation in density of PyC fragments: } & \multicolumn{5}{|c|}{0.0076} \\
\hline \multicolumn{4}{|c|}{ Uncertainty in calculated density of PyC fragments: } & \multicolumn{5}{|c|}{0.0016} \\
\hline
\end{tabular}

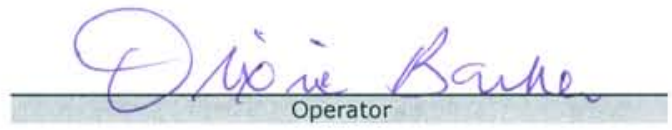

$12,21-05$ 
Data Report Form DRF-18A: Measurement of Pyrocarbon Anisotropy using the 2-MGEM - IPyC

\begin{tabular}{|r|l|}
\hline Procedure: & AGR-CHAR-DAM-18 Rev. 1 \\
\hline Operator: & G. E. Jellison \\
\hline Mount ID: & M06011102L \\
\hline Sample ID: & LEU01-46T-B01 \\
\hline Sample Description: & Baseline Composite of TRISO on BWXT kernel composite 69302 \\
\hline Folder containing data: & IIMc-agr\AGR\2-MGEM\R06030601\} $\\
{\hline}$
\end{tabular}

\begin{tabular}{|c|c|c|c|c|c|c|c|}
\hline \multirow{2}{*}{ Particle \# } & \multirow{2}{*}{$\begin{array}{c}\text { Grid } \\
\text { Position }\end{array}$} & \multicolumn{3}{|c|}{ Diattenuation } & \multicolumn{3}{|c|}{ Equivalent BAFo } \\
\hline & & Average & St. Dev. & Ave. Error & Average & St. Dev. & Ave. Error \\
\hline 1 & 4,4 & 0.0075 & 0.0017 & 0.0012 & 1.0225 & 0.0051 & 0.0036 \\
\hline 2 & 4,6 & 0.0066 & 0.0023 & 0.0012 & 1.0198 & 0.0069 & 0.0036 \\
\hline 3 & 4,7 & 0.0081 & 0.0030 & 0.0012 & 1.0243 & 0.0090 & 0.0036 \\
\hline 4 & 5,4 & 0.0085 & 0.0023 & 0.0011 & 1.0255 & 0.0069 & 0.0033 \\
\hline 5 & 5,5 & 0.0069 & 0.0020 & 0.0011 & 1.0207 & 0.0060 & 0.0033 \\
\hline 6 & 5,6 & 0.0077 & 0.0023 & 0.0014 & 1.0231 & 0.0069 & 0.0042 \\
\hline 7 & 5,7 & 0.0063 & 0.0025 & 0.0014 & 1.0189 & 0.0075 & 0.0042 \\
\hline 8 & 6,4 & 0.0080 & 0.0021 & 0.0015 & 1.0240 & 0.0063 & 0.0045 \\
\hline 9 & 6,5 & 0.0069 & 0.0019 & 0.0014 & 1.0207 & 0.0057 & 0.0042 \\
\hline 10 & 6,6 & 0.0075 & 0.0024 & 0.0012 & 1.0225 & 0.0072 & 0.0036 \\
\hline \multicolumn{2}{|c|}{ Average } & 0.0074 & 0.0023 & 0.0013 & 1.0222 & 0.0068 & 0.0038 \\
\hline
\end{tabular}

Mean of average BAFo per particle: 11.0222

Standard deviation of average BAFo per particle: 0.0021

\section{Comments}
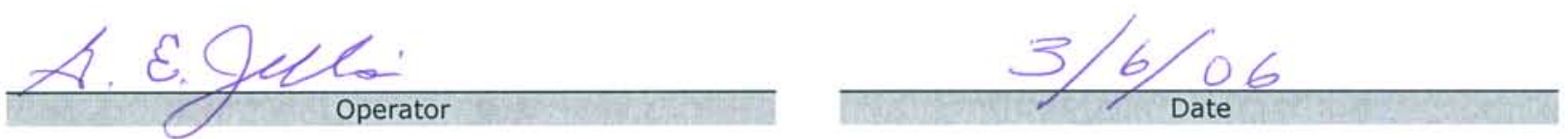
Data Report Form DRF-18B: Measurement of Pyrocarbon Anisotropy using the 2-MGEM - OPyC

\begin{tabular}{|r|l|}
\hline Procedure: & AGR-CHAR-DAM-18 Rev. 1 \\
\hline Operator: & G. E. Jellison \\
\hline Mount ID: & M06011102L \\
\hline Sample ID: & LEU01-46T-B01 \\
\hline Sample Description: & Baseline Composite of TRISO on BWXT kernel composite 69302 \\
\hline Folder containing data: & $\backslash \backslash$ MC-agr\AGR\2-MGEM\R06030601\} $\\
{\hline}$
\end{tabular}

\begin{tabular}{|c|c|c|c|c|c|c|c|}
\hline \multirow{2}{*}{ Particle \# } & \multirow{2}{*}{$\begin{array}{c}\text { Grid } \\
\text { Position }\end{array}$} & \multicolumn{3}{|c|}{ Diattenuation } & \multicolumn{3}{c|}{ Equivalent BAFo } \\
\cline { 3 - 8 } & Average & St. Dev. & Ave. Error & Average & St. Dev. & Ave. Error \\
\hline \hline 1 & 4,4 & 0.0068 & 0.0022 & 0.0013 & 1.0204 & 0.0066 & 0.0039 \\
\hline 2 & 4,6 & 0.0056 & 0.0023 & 0.0012 & 1.0168 & 0.0069 & 0.0036 \\
\hline 3 & 4,7 & 0.0056 & 0.0020 & 0.0012 & 1.0168 & 0.0060 & 0.0036 \\
\hline 4 & 5,4 & 0.0056 & 0.0019 & 0.0012 & 1.0168 & 0.0057 & 0.0036 \\
\hline 5 & 5,5 & 0.0060 & 0.0021 & 0.0015 & 1.0180 & 0.0063 & 0.0045 \\
\hline 6 & 5,6 & 0.0069 & 0.0024 & 0.0014 & 1.0207 & 0.0072 & 0.0042 \\
\hline 7 & 5,7 & 0.0077 & 0.0031 & 0.0016 & 1.0231 & 0.0093 & 0.0048 \\
\hline 8 & 6,4 & 0.0076 & 0.0022 & 0.0016 & 1.0228 & 0.0066 & 0.0048 \\
\hline 9 & 6,5 & 0.0053 & 0.0020 & 0.0015 & 1.0159 & 0.0060 & 0.0045 \\
\hline 10 & 6,6 & 0.0062 & 0.0024 & 0.0012 & 1.0186 & 0.0072 & 0.0036 \\
\hline \multicolumn{2}{|r|}{ Average } & 0.0063 & 0.0023 & 0.0014 & 1.0190 & 0.0068 & 0.0041 \\
\hline
\end{tabular}

Mean of average BAFo per particle: 1.0190

Standard deviation of average BAFo per particle: 0.0026

\section{Comments}
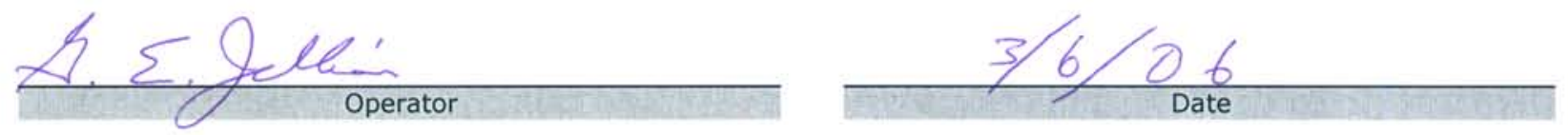
Data Report Form DRF-20: Counting of Particles with SiC Gold Spot Defects by Visual Inspection

\begin{tabular}{|r|l|}
\hline Procedure: & AGR-CHAR-DAM-20 Rev. 1 \\
\hline Operator: & John Hunn \\
\hline Sample ID: & LEU01-46T-D01 \\
\hline Sample Description: & Baseline Composite TRISO on BWXT kernel composite 69302 \\
\hline Filename: & IImc-agr\AGR\GoldSpots\G06011101_DRF20R1.xls \\
\hline
\end{tabular}

\begin{tabular}{|r|l|}
\hline Mean average weight/particle $(g):$ & $7.27 \mathrm{E}-04$ \\
\hline Uncertainty in average weight/particle $(\mathrm{g}):$ & $4.25 \mathrm{E}-07$ \\
\hline Weight of sample of particles $(\mathrm{g}):$ & 22.702 \\
\hline Approximate number of particles in sample: & 31227 \\
\hline Uncertainty in number of particles in sample: & 18 \\
\hline
\end{tabular}

Number of particles with gold spot defects: 27

\section{Comments on unusual visual characteristics of SiC}

Stage 1 and 2 sampling skipped, analyzed all available particles in missing OPyC sample after completion of DAM-19.

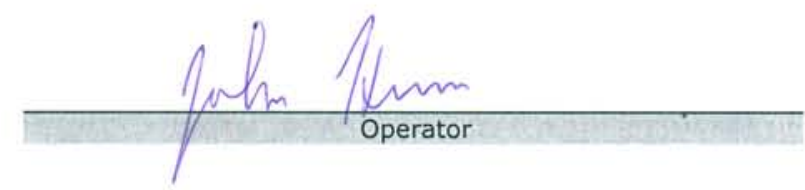

$$
1-11-06
$$


27/31227 gold spots found.

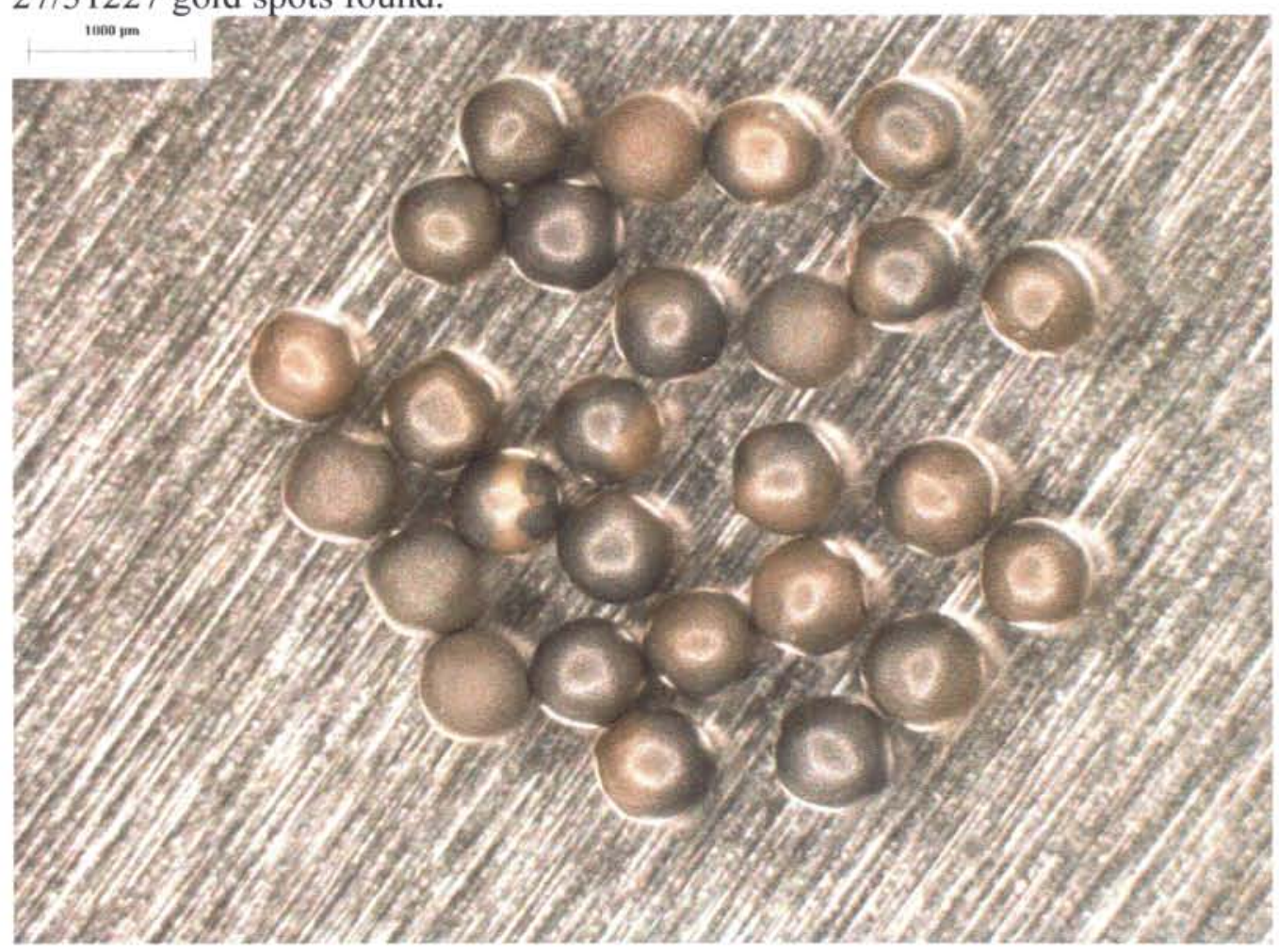

Cross section of gold spot showing carbon soot inclusion at IPyC outer surface.

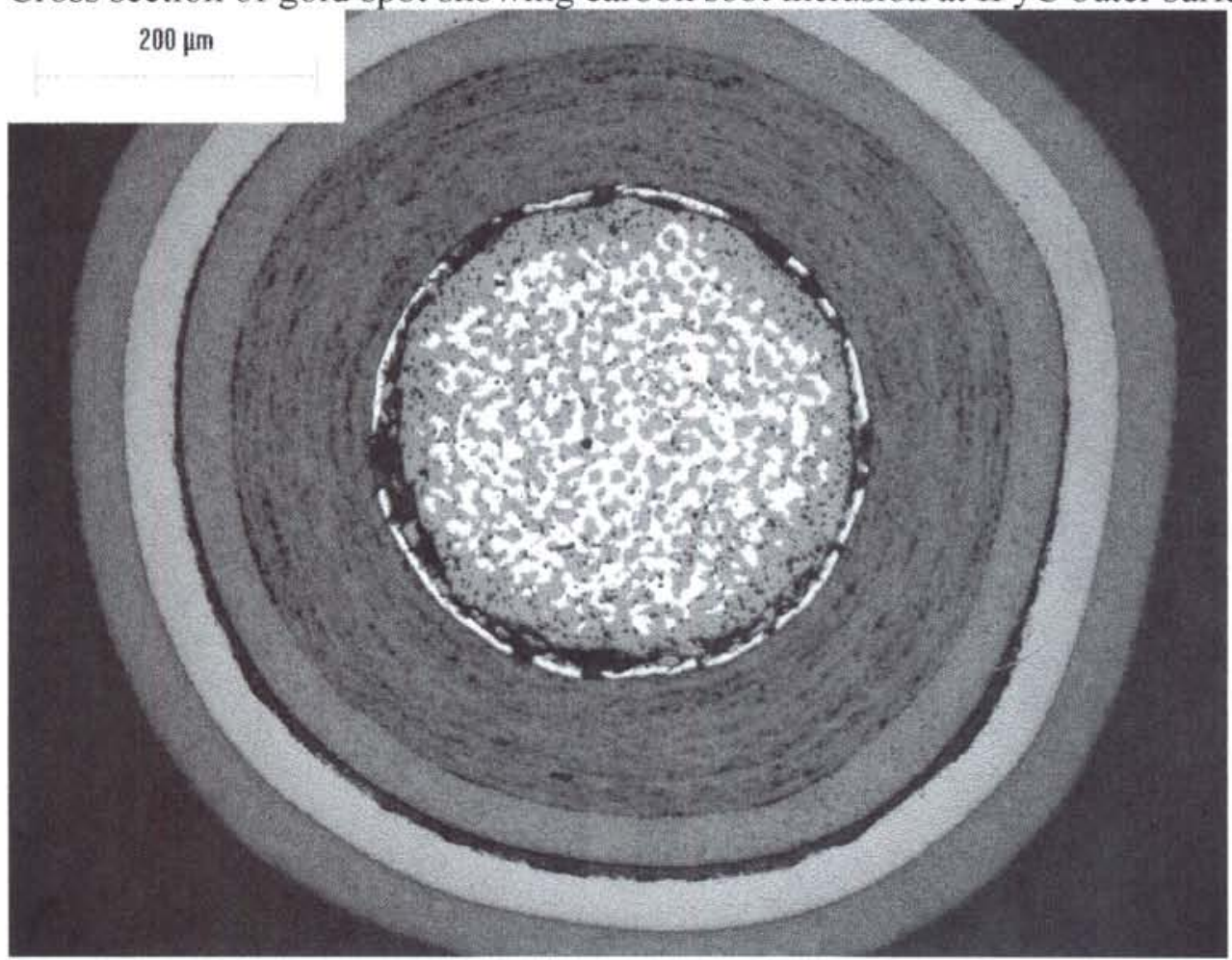


ORNL/TM-2006/019, Rev. 1

Data Report Form DRF-20: Counting of Particles with SiC Gold Spot Defects by Visual Inspection

\begin{tabular}{|r|l|}
\hline Procedure: & AGR-CHAR-DAM-20 Rev. 1 \\
\hline Operator: & John Hunn \\
\hline Sample ID: & LEU01-46T-F01 \\
\hline Sample Description: & Baseline TRISO on BWXT kernel composite 69302 \\
\hline Filename: & IImc-agr\AGRIGoldSpots\G06012002_DRF20R1.xIs \\
\hline
\end{tabular}

Mean average weight/particle (g): $7.27 \mathrm{E}-04$

Uncertainty in average weight/particle (g): $4.25 \mathrm{E}-07$

Weight of sample of particles $(\mathrm{g}): 36.553$

Approximate number of particles in sample: 50280

Uncertainty in number of particles in sample: 29

Number of particles with gold spot defects: 39

\section{Comments on unusual visual characteristics of SiC}

This sample was analyzed for gold spots after performing burn-leach per DAM-21.

Some brown colored particles were observed. Examination in cross section showed that this brown surface coloration was not related to any visible defect in the SiC. They were not counted as having gold spot defects.

One particle was white on half of surface. This appeared to be due to surface roughness.

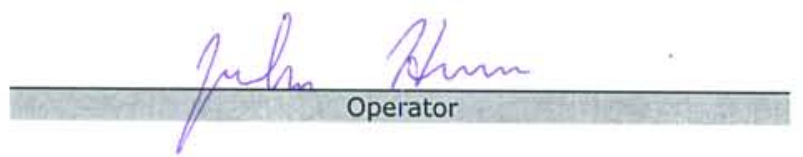

$1-20-06$

$\frac{1-20-06}{\text { Date }}$


$39 / 50280$ gold spots found.

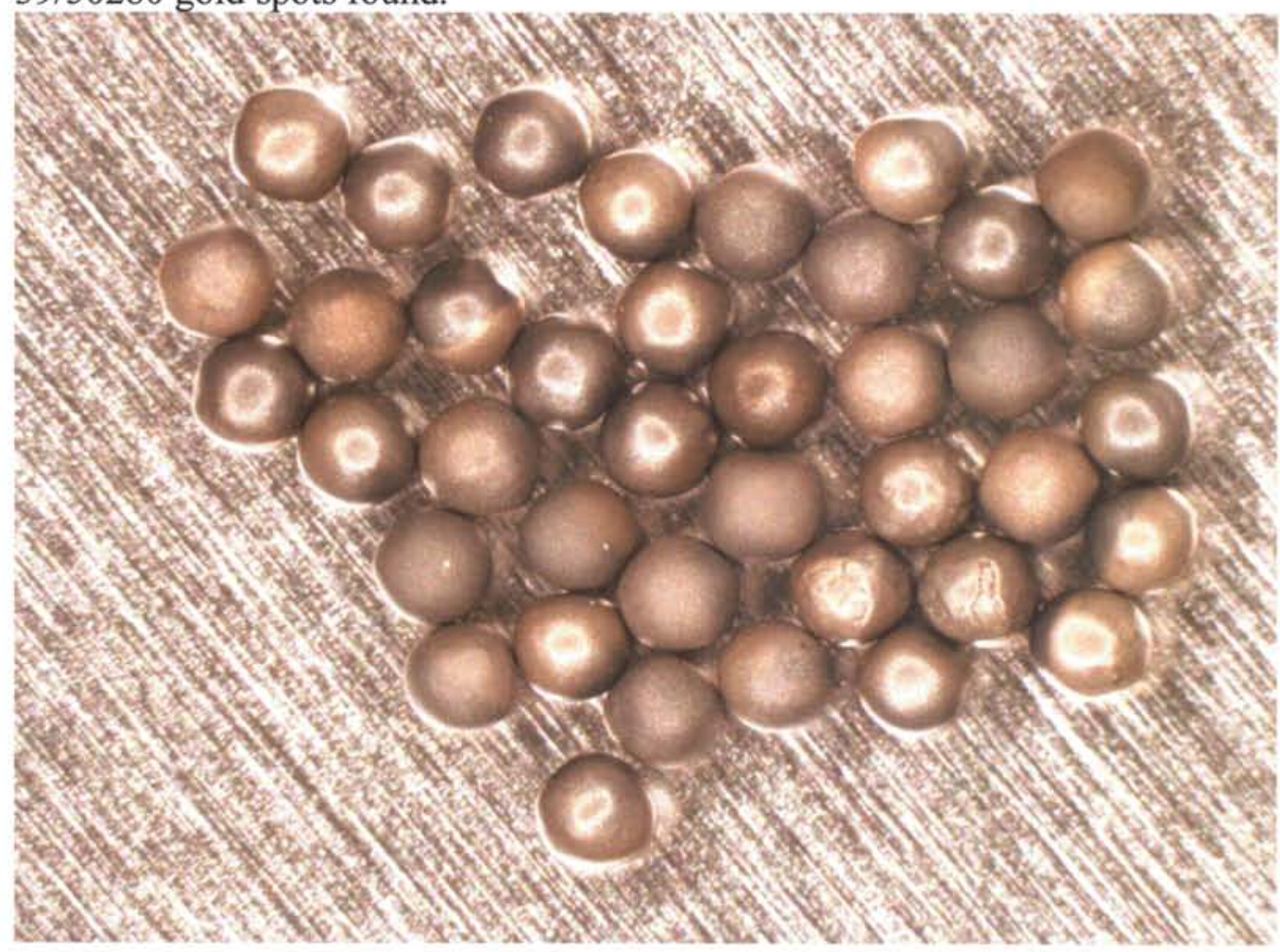

Brown surface color observed on some particle after burn-leach process. This appearance was not associated with gold spot type defects. White coating was also a surface effect.

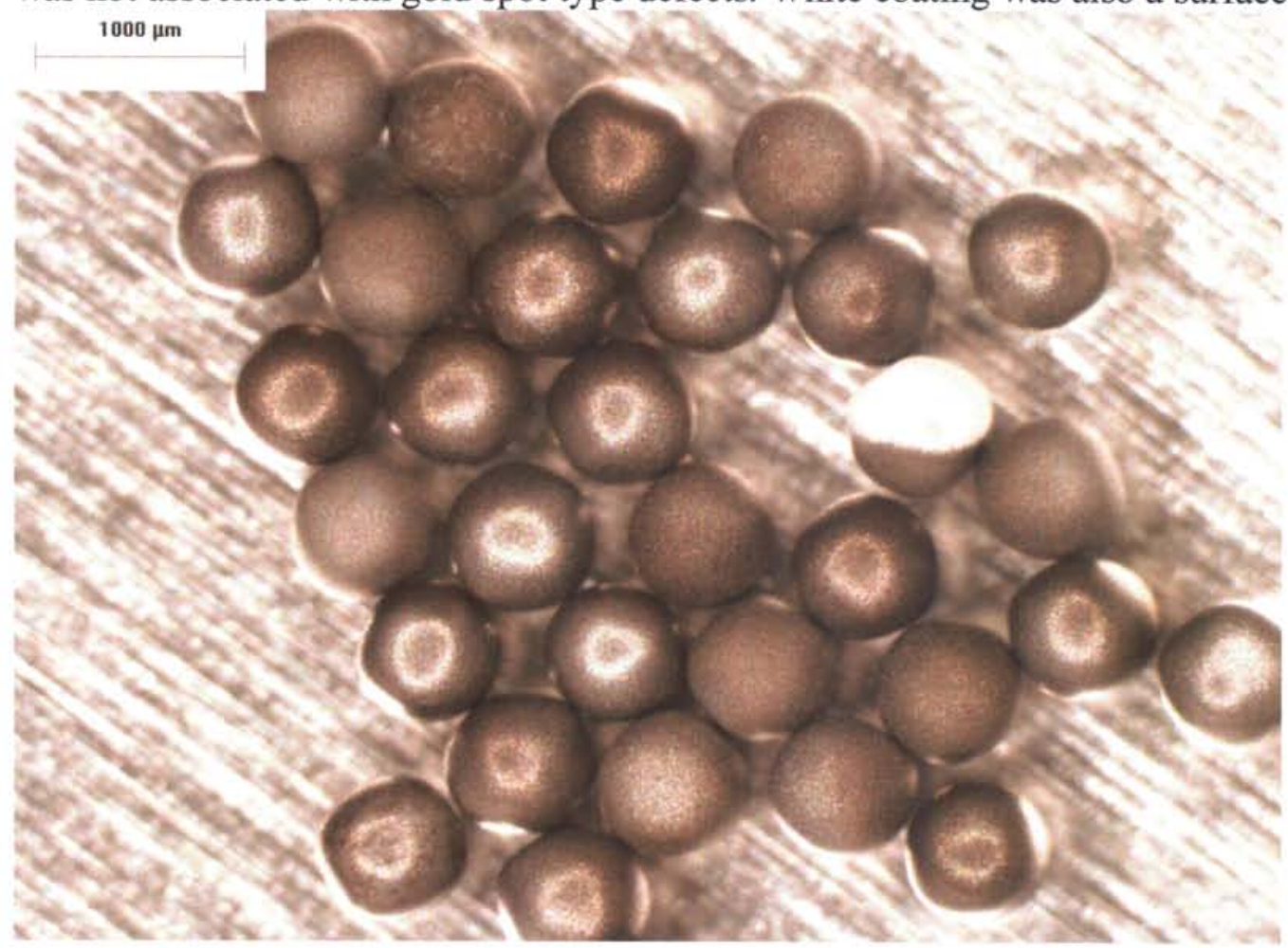


Data Report Form DRF-07: Imaging of Particle Diameter and Aspect Ratio Using an Optical Microscope System

Procedure: AGR-CHAR-DAM-07 Rev. 1

Operator: John Hunn

Sample ID: LEU01-46T-C01

Sample Description: Baseline composite of TRISO on BWXT kernel composite 69302

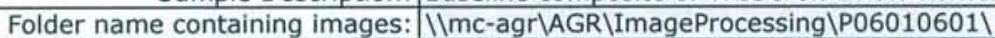

DMR Calibration Expiration Date: $9 / 8 / 06$

Stage Micrometer Calibration Expiration Date: $2 / 17 / 06$

Measured Value for $1200 \mu \mathrm{m}$ in Stage Micrometer Image: $1200 . \mu \mathrm{m}$

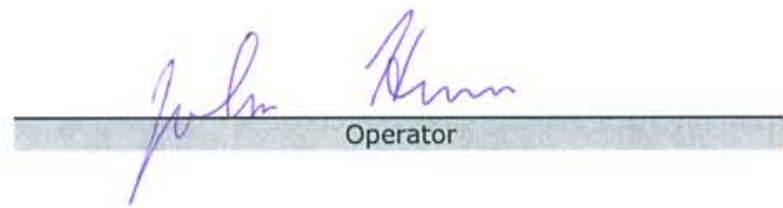

$$
1-6-06
$$

Date 


\begin{tabular}{|c|c|}
\hline Procedure: & AGR-CHAR-DAM-10 Rev. 2 \\
\hline Operator: & Andrew K. Kercher \\
\hline Folder name containing images: & 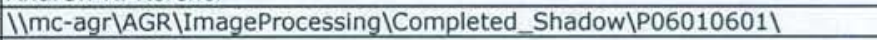 \\
\hline Sample ID: & LEU01-46T-C01 \\
\hline Sample Description: & Baseline Composite of TRISO on BWXT kernel composite 69302 \\
\hline
\end{tabular}

\begin{tabular}{|r|r|}
\hline Number of particles analyzed: & 1626 \\
\hline Number of particles with aspect ratio $\geq 1.14$ & 2 \\
\hline Average particle aspect ratio: & 1.054 \\
\hline
\end{tabular}

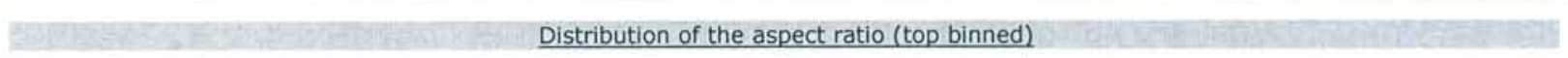

\begin{tabular}{|c|c|}
\hline Aspect Ratio $(D)$ & Frequency \\
\hline 1.005 & 0 \\
\hline 1.010 & 0 \\
\hline 1.015 & 3 \\
\hline 1.020 & 14 \\
\hline 1.025 & 32 \\
\hline 1.030 & 64 \\
\hline 1.035 & 107 \\
\hline 1.040 & 148 \\
\hline 1.045 & 177 \\
\hline 1.050 & 176 \\
\hline 1.055 & 181 \\
\hline 1.060 & 163 \\
\hline 1.065 & 147 \\
\hline 1.070 & 112 \\
\hline 1.075 & 89 \\
\hline 1.080 & 57 \\
\hline 1.085 & 43 \\
\hline 1.090 & 54 \\
\hline 1.095 & 19 \\
\hline 1.100 & 19 \\
\hline 1.105 & 6 \\
\hline 1.110 & 4 \\
\hline 1.115 & 6 \\
\hline 1.120 & 1 \\
\hline 1.125 & 1 \\
\hline 1.130 & 0 \\
\hline 1.135 & 0 \\
\hline 1.140 & 1 \\
\hline$>1.14$ & 2 \\
\hline
\end{tabular}
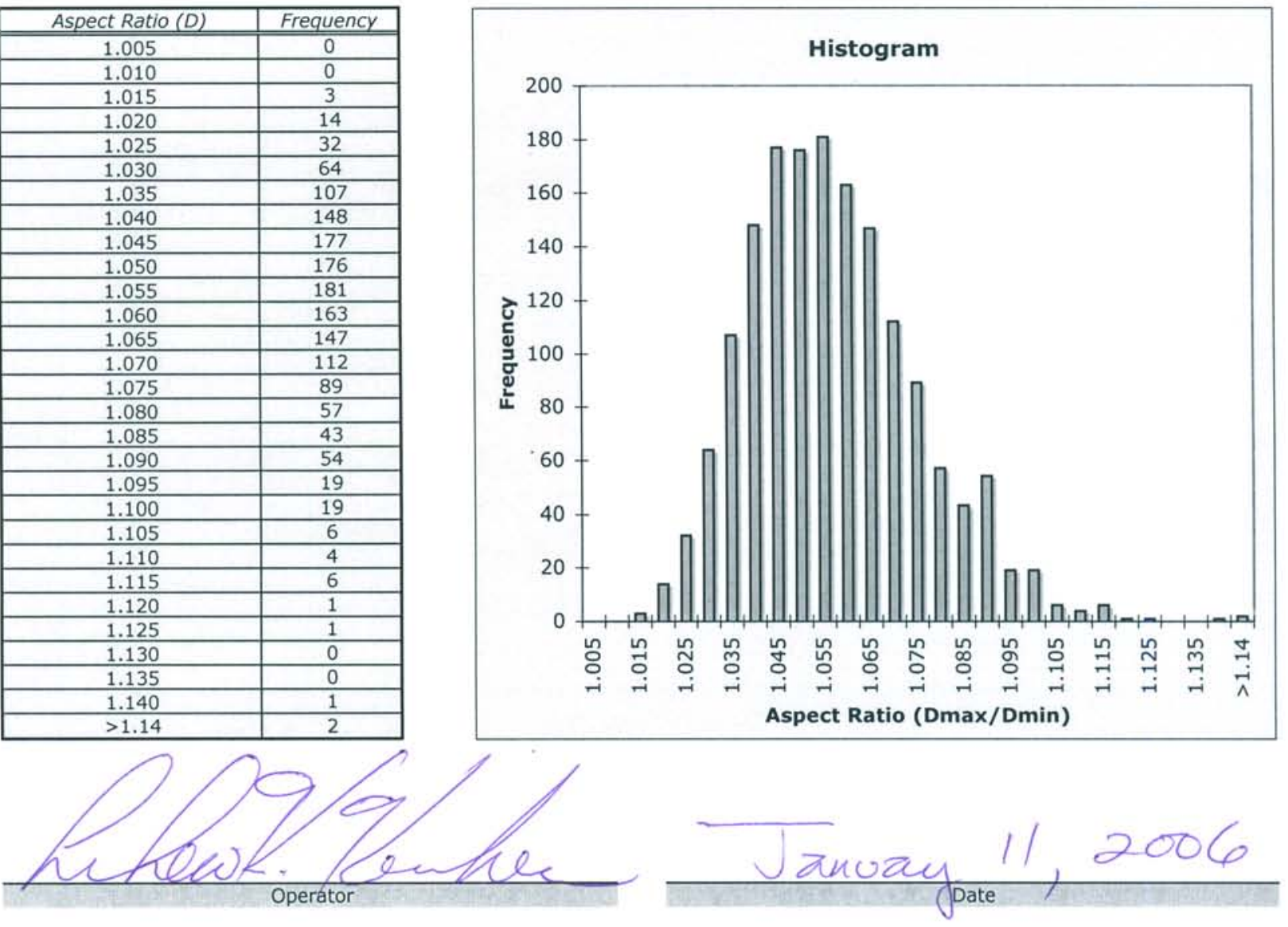
Data Report Form DRF-21: Measurement of Number of Particles with SiC Burn-Leach Defects

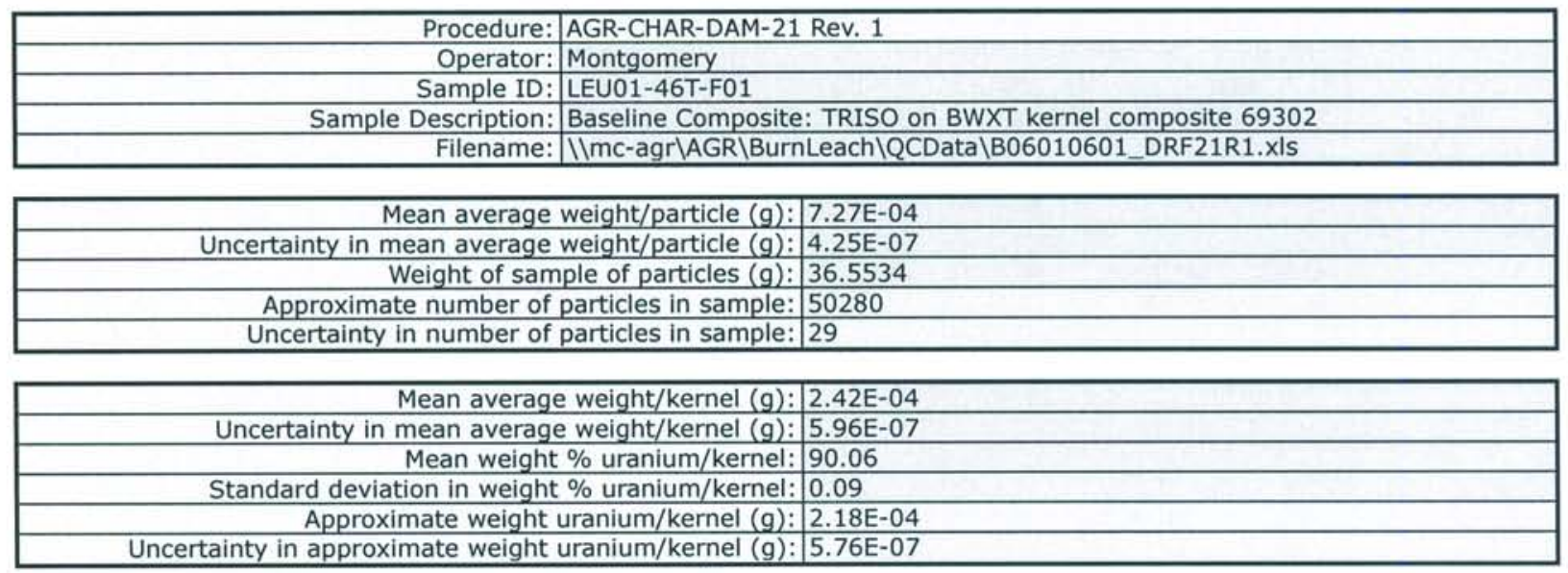

\begin{tabular}{|c|c|c|c|c|}
\hline & Leach 1 & Leach 2 & Leach 3 & Total \\
\hline Sample ID & B0601060101 & B0601180102 & & \\
\hline Volume of solution $(\mathrm{ml})$ : & 44.8 & 49.5 & & \\
\hline Measured $\beta$ activity of $0.1 \mathrm{ml}$ aliquot (dpm): & 0.0 & 0.0 & & \\
\hline Estimated weight of $\mathrm{U}$ in solution $(\mathrm{mg})$ : & 0 & 0 & 0 & \\
\hline Radiochemical laboratory analysis number & $060223-019$ & $060223-021$ & & \\
\hline Weight uranium leached $(g)$ : & $2.79 \mathrm{E}-06$ & 3.27E-07 & & $3.12 \mathrm{E}-06$ \\
\hline Uncertainty in weight uranium leached $(g)$ : & $2.80 \mathrm{E}-07$ & $3.30 \mathrm{E}-08$ & & $2.82 \mathrm{E}-07$ \\
\hline Calculated number of particles with SiC defects: & 0 & 0 & & 0 \\
\hline Uncertainty in number of particles with SiC defects: & $1.29 \mathrm{E}-03$ & $1.52 \mathrm{E}-04$ & & $1.30 \mathrm{E}-03$ \\
\hline
\end{tabular}

\section{Comments}

Examined particles under microscope after completion of burn-leach. 31 gold spot defects were identified. No particles appeared hollow. No SiC fragments were observed.

Fcm Checked data 3/01/06

Zaed c. Montgomery
$3 / 20 / 06$

Date 
Data Report Form DRF-21: Measurement of Number of Particles with SiC Burn-Leach Defects

\begin{tabular}{|c|c|}
\hline Procedure: & AGR-CHAR-DAM-21 Rev. 1 \\
\hline Operator: & Montgomery \\
\hline Sample ID: & LEU01-46T-G01 \\
\hline Sample Description: & Baseline Composite: TRISO on BWXT kernel composite 69302 \\
\hline Filename: & 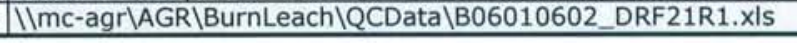 \\
\hline
\end{tabular}

\begin{tabular}{|r|l|}
\hline Mean average weight/particle $(g):$ & $7.27 \mathrm{E}-04$ \\
\hline Uncertainty in mean average weight/particle $(g):$ & $4.25 \mathrm{E}-07$ \\
\hline Weight of sample of particles $(\mathrm{g}):$ & 51.1864 \\
\hline Approximate number of particles in sample: & 70408 \\
\hline Uncertainty in number of particles in sample: & 41 \\
\hline
\end{tabular}

\begin{tabular}{|r|l|}
\hline Mean average weight/kernel $(g):$ & $2.42 \mathrm{E}-04$ \\
\hline Uncertainty in mean average weight/kernel $(\mathrm{g}):$ & $5.96 \mathrm{E}-07$ \\
\hline Mean weight \% uranium/kernel: & 90.06 \\
\hline Standard deviation in weight \% uranium/kernel: & 0.09 \\
\hline Approximate weight uranium/kernel $(\mathrm{g}):$ & $2.18 \mathrm{E}-04$ \\
\hline Uncertainty in approximate weight uranium/kernel $(\mathrm{g}):$ & $5.76 \mathrm{E}-07$ \\
\hline
\end{tabular}

\begin{tabular}{|c|c|c|c|c|}
\hline & Leach 1 & Leach 2 & Leach 3 & Total \\
\hline Sample ID & B0601060201 & B0601180202 & & \\
\hline Volume of solution $(\mathrm{ml})$ : & 44.5 & 45.0 & & \\
\hline Measured $\beta$ activity of $0.1 \mathrm{ml}$ aliquot (dpm): & 0.0 & 0.0 & & \\
\hline Estimated weight of $U$ in solution $(\mathrm{mg})$ : & 0 & 0 & 0 & \\
\hline Radiochemical laboratory analysis number & $060223-020$ & $060223-022$ & & \\
\hline Weight uranium leached $(\mathrm{g})$ : & $4.73 \mathrm{E}-06$ & 4.07E-07 & & $5.14 \mathrm{E}-06$ \\
\hline Uncertainty in weight uranium leached $(\mathrm{g})$ : & $4.70 \mathrm{E}-07$ & $4.10 \mathrm{E}-08$ & & $4.72 E-07$ \\
\hline Calculated number of particles with SiC defects: & 0 & 0 & & 0 \\
\hline Uncertainty in number of particles with SiC defects: & $2.16 \mathrm{E}-03$ & $1.88 \mathrm{E}-04$ & & 2.17E-03 \\
\hline
\end{tabular}

\section{Comments}

fcm checked data $3 / 01 / 06$
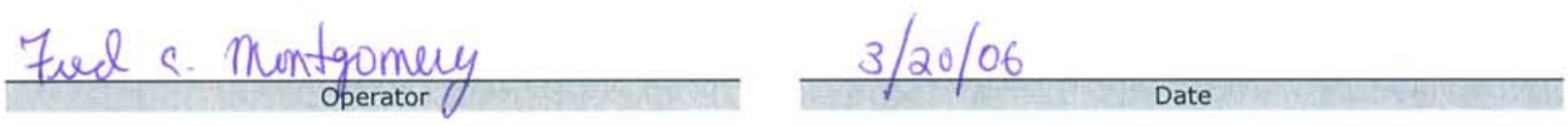
Data Report Form DRF-19: Counting of Particles with Missing OPyC Layer by Visual Inspection

\begin{tabular}{|r|l|}
\hline Procedure: & AGR-CHAR-DAM-19 Rev. 1 \\
\hline Operator: & John Hunn \\
\hline Sample ID: & LEU01-46T-D01 \\
\hline Sample Description: & Baseline Composite TRISO on BWXT kernel composite 69302 \\
\hline Filename: & IImC-agr\AGR\MissingOPyCIX06011001_DRF19R1.xls \\
\hline
\end{tabular}

\begin{tabular}{rl|}
\hline Mean average weight/particle $(g):$ & $7.27 \mathrm{E}-04$ \\
\hline Uncertainty in average weight/particle $(\mathrm{g}):$ & $4.25 \mathrm{E}-07$ \\
\hline Weight of sample of particles $(\mathrm{g}):$ & 22.702 \\
\hline Approximate number of particles in sample: & 31227 \\
\hline Uncertainty in number of particles in sample: & 18 \\
\hline
\end{tabular}

Comments on unusual visual characteristics of OPyC

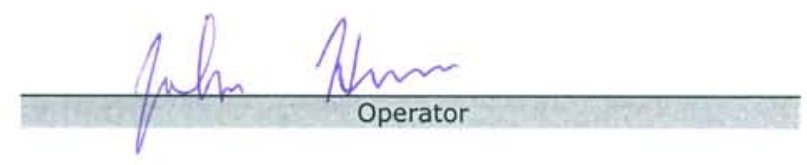

$$
1-10-06
$$


Data Report Form DRF-23: Imaging of SiC Grain Structure

\begin{tabular}{|r|l|}
\hline Procedure: & AGR-CHAR-DAM-23 Rev. 0 \\
\hline Operator: & Paul Menchhofer \\
\hline Filename: & IImc-agr\AGRISEMIE06011101_DRF23R0.xIs \\
\hline Sample ID: & LEU01-46T-B01 \\
\hline Sample Description: & Baseline Composite of TRISO on BWXT kernel composite 69302 \\
\hline Mount Number(s): & M06011001L \\
\hline Folder name containing images: & \Imc-agr\AGRISEMI \\
\hline
\end{tabular}

\begin{tabular}{|c|c|c|c|}
\hline \multicolumn{4}{|c|}{ Images of SiC grain structure } \\
\hline & particle 1 & particle 2 & particle 3 \\
\hline 1000x image filename & 57262 & 57267 & 57269 \\
\hline 2500x image filename & 57264 & 57268 & 57270 \\
\hline \hline grain structure acceptable & Yes & Yes & Yes \\
\hline
\end{tabular}
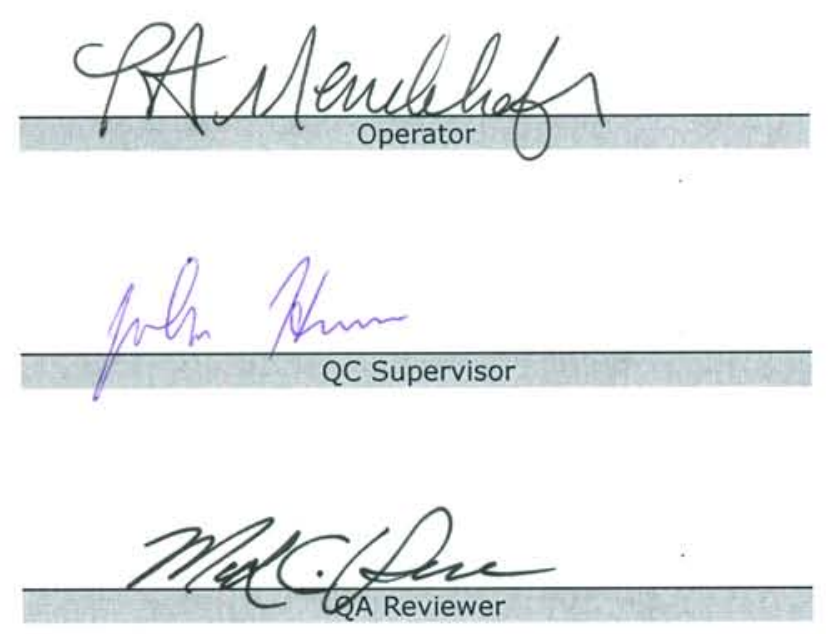

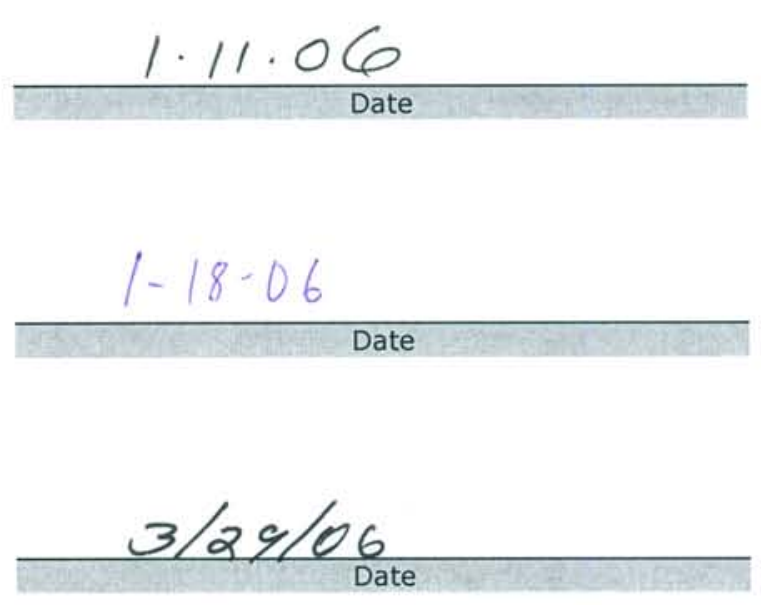




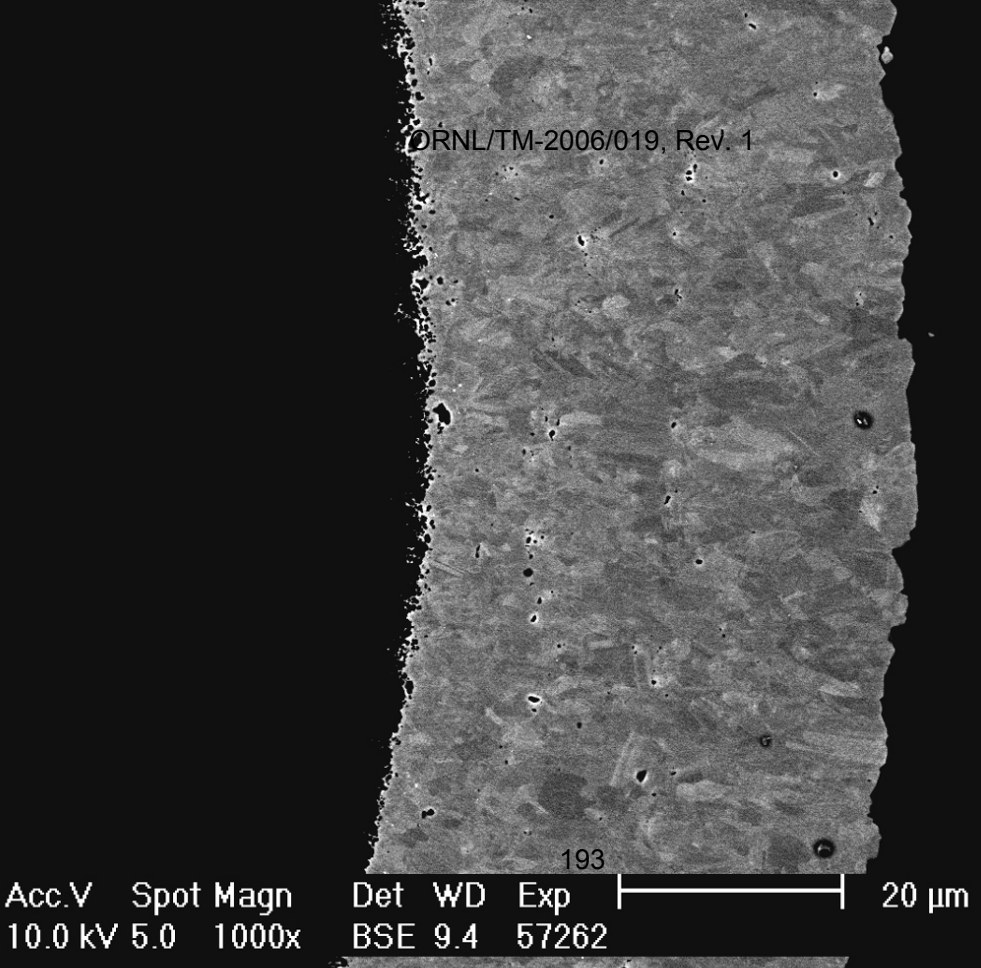




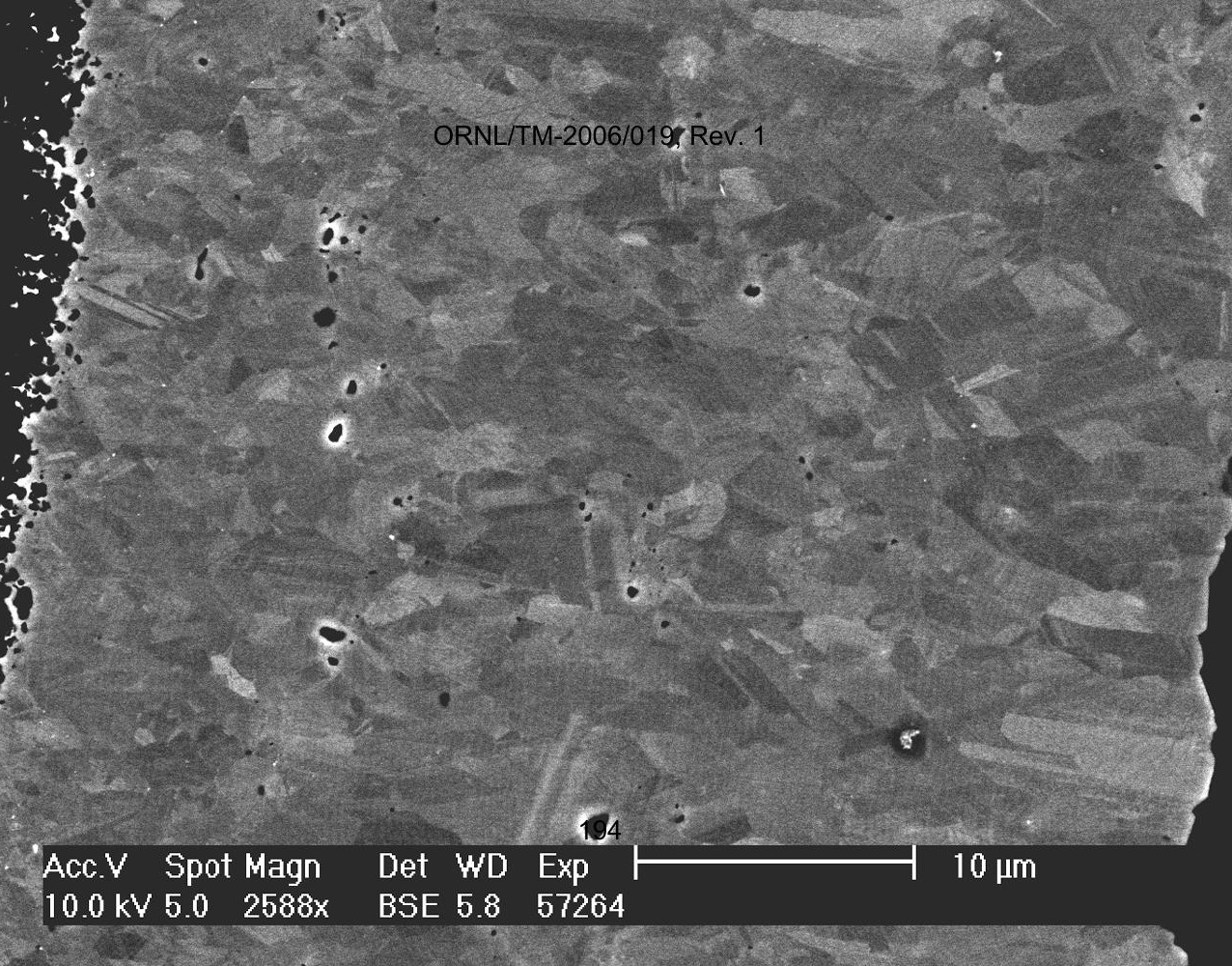




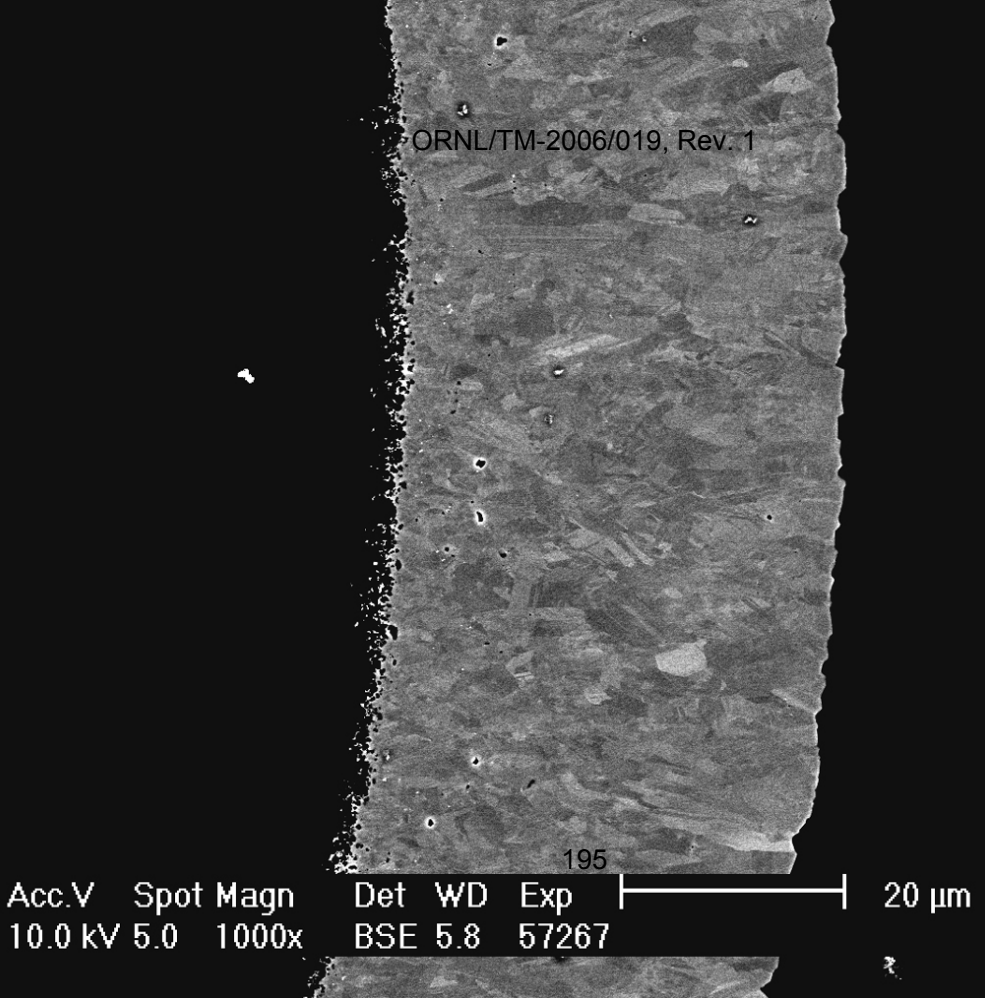




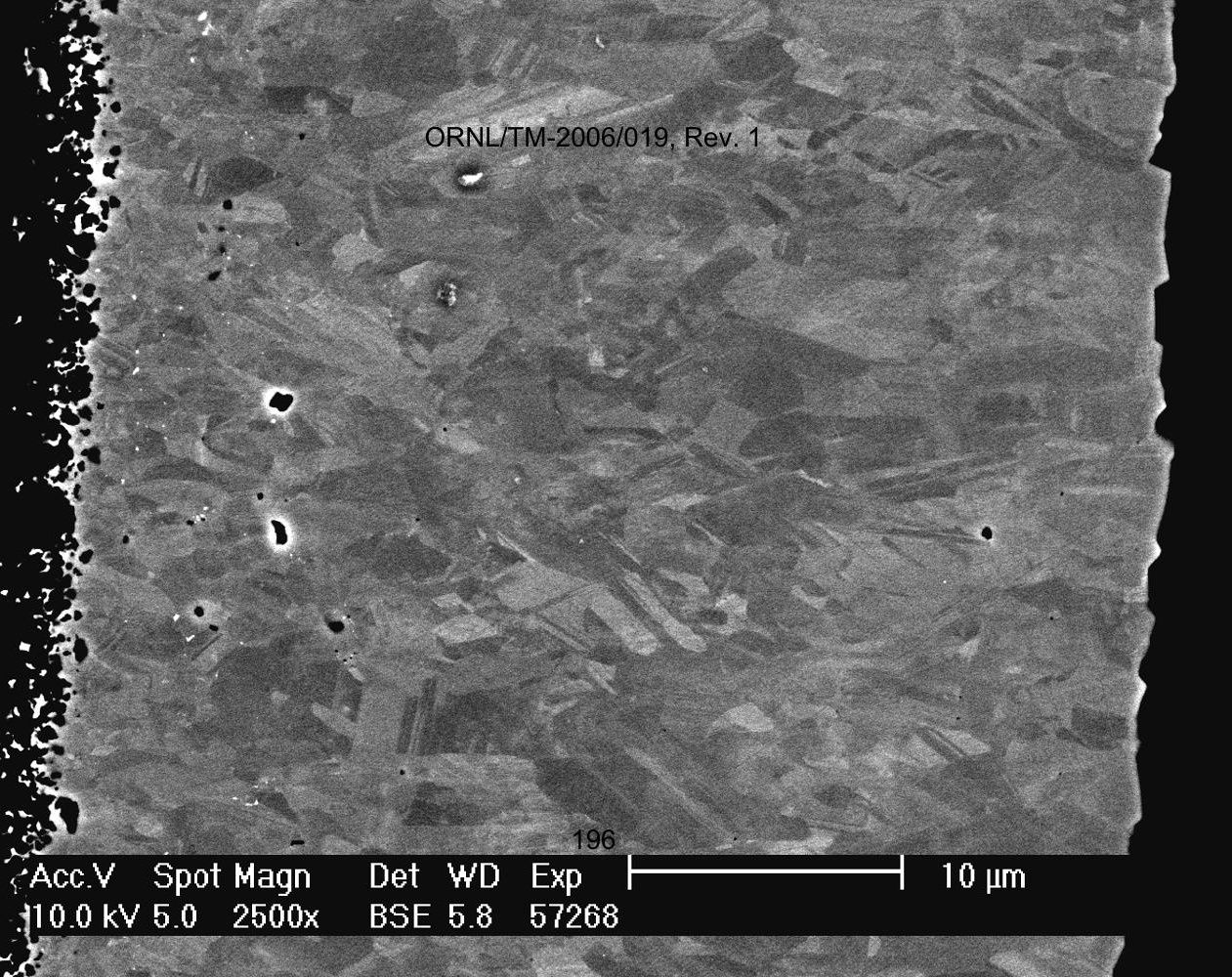




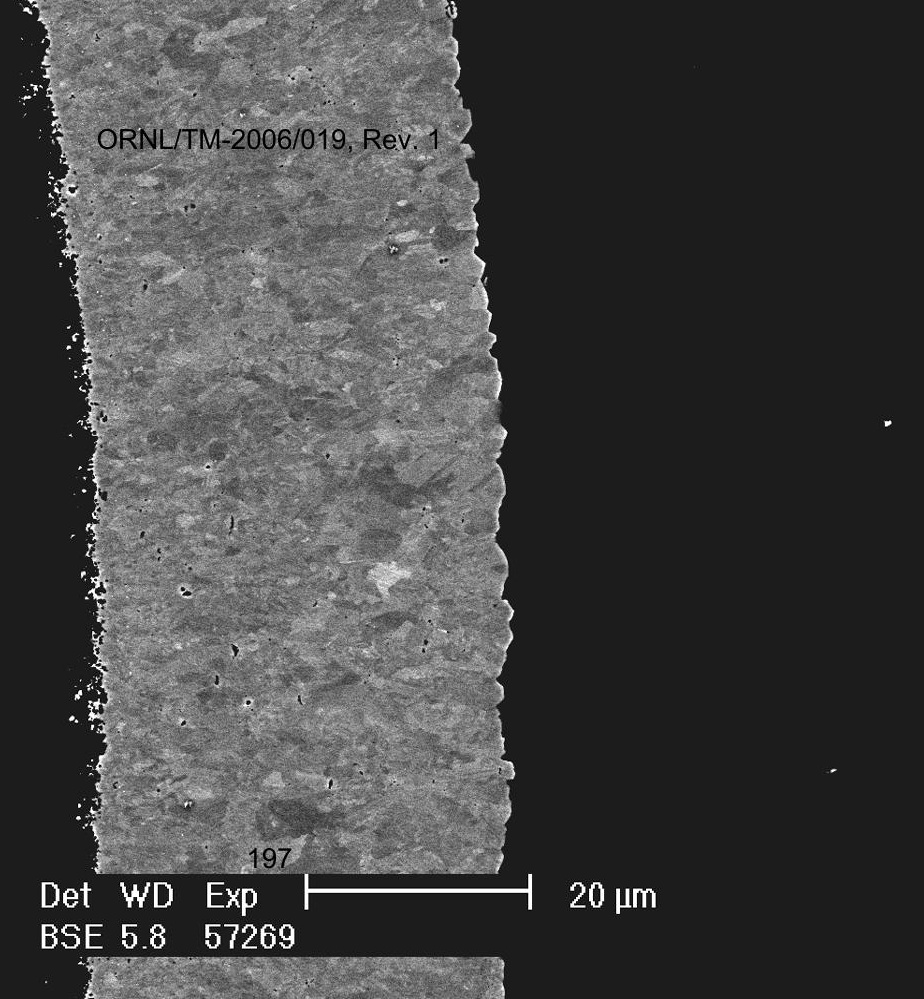




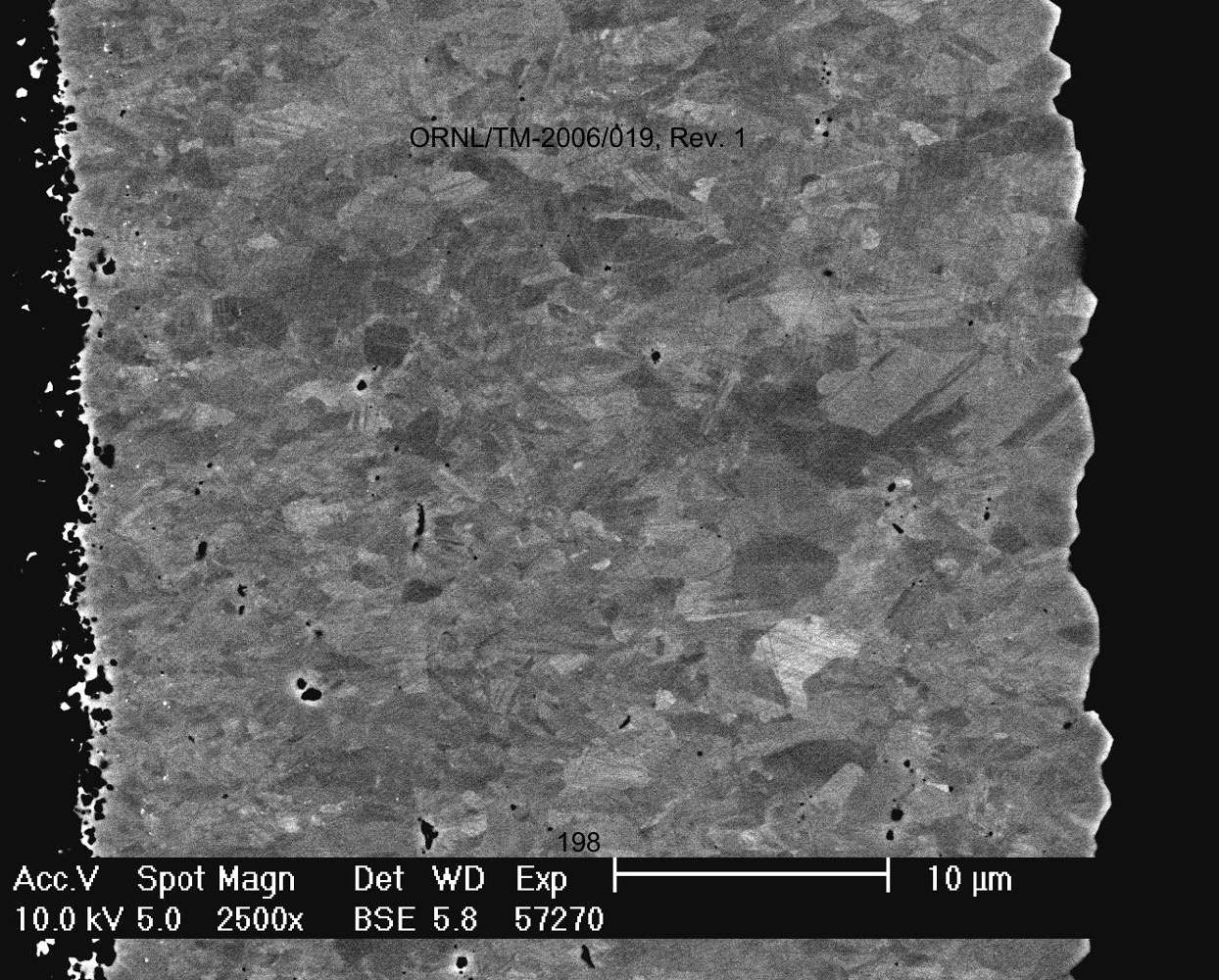




\section{For Information Only}

The information in the remainder of this section reports results of measurements not required by the fuel specification and is provided for information only. 
Data Report Form DRF-22: Estimation of Average Particle Weight

\begin{tabular}{|r|l|}
\hline Procedure: & AGR-CHAR-DAM-22 Rev. 1 \\
\hline Operator: & Dixie Barker \\
\hline Particle Lot ID: & LEU01-46T-H01 \\
\hline Particle Lot Description: & TRISO on BWXT kernel composite 69302 \\
\hline Filename: & IIMC-agr\AGR\ParticleWeight|W06010501_DRF22R1.xIs \\
\hline
\end{tabular}

\begin{tabular}{|r|c|c|c|c|c|}
\hline & Sample 1 & Sample 2 & Sample 3 & Sample 4 & Sample 5 \\
\hline Weight of particles (g): & $9.29 \mathrm{E}-02$ & $8.66 \mathrm{E}-02$ & $8.81 \mathrm{E}-02$ & $1.09 \mathrm{E}-01$ & $9.88 \mathrm{E}-02$ \\
\hline Number of particles: & 128 & 119 & 121 & 150 & 136 \\
\hline Average weight/particle (g): & $7.26 \mathrm{E}-04$ & $7.28 \mathrm{E}-04$ & $7.28 \mathrm{E}-04$ & $7.27 \mathrm{E}-04$ & $7.26 \mathrm{E}-04$ \\
\hline
\end{tabular}

Mean average weight/particle (g): $7.27 \mathrm{E}-04$

Uncertainty in mean average weight/particle $(g): 4.25 \mathrm{E}-07$
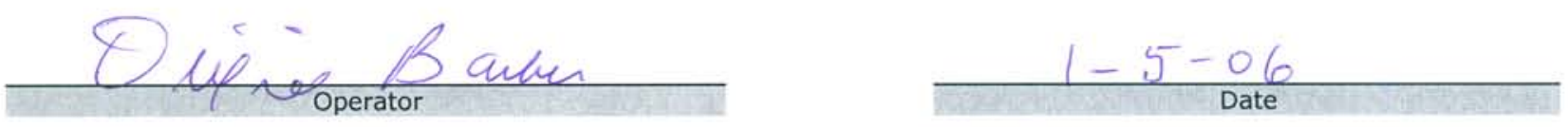
Procedure: AGR-CHAR-DAM-10 Rev. 2

Operator: Andrew K. Kercher

Folder name containing images: |Imc-agr|AGR\ImageProcessing|Completed_Shadow\P06010601 Sample ID: LEU01-46T-C01

Sample Description: Baseline Composite of TRISO on BWXT kernel composite 69302

Folder name containing processed data: \Imc-agr|AGR\ImageProcessing|Completed_Shadow\P06010601_output|

\begin{tabular}{|r|c|}
\hline Number of particles analyzed: & 1626 \\
\hline Mean of the average diameter of each particle $(\mu \mathrm{m}):$ & 799.7 \\
\hline Standard deviation in the average diameter of each particle $(\mu \mathrm{m}):$ & 14 \\
\hline
\end{tabular}

\section{Distribution of the average particle diameter (top binned)}

\begin{tabular}{|c|c|}
\hline Mean Diameter $(\mu \mathrm{m})$ & Frequency \\
\hline \hline$<700$ & 0 \\
\hline 710 & 0 \\
\hline 720 & 0 \\
\hline 730 & 0 \\
\hline 740 & 0 \\
\hline 750 & 0 \\
\hline 760 & 2 \\
\hline 770 & 20 \\
\hline 780 & 110 \\
\hline 790 & 269 \\
\hline 800 & 428 \\
\hline 810 & 419 \\
\hline 820 & 271 \\
\hline 830 & 78 \\
\hline 840 & 24 \\
\hline 850 & 4 \\
\hline$>850$ & 1 \\
\hline
\end{tabular}
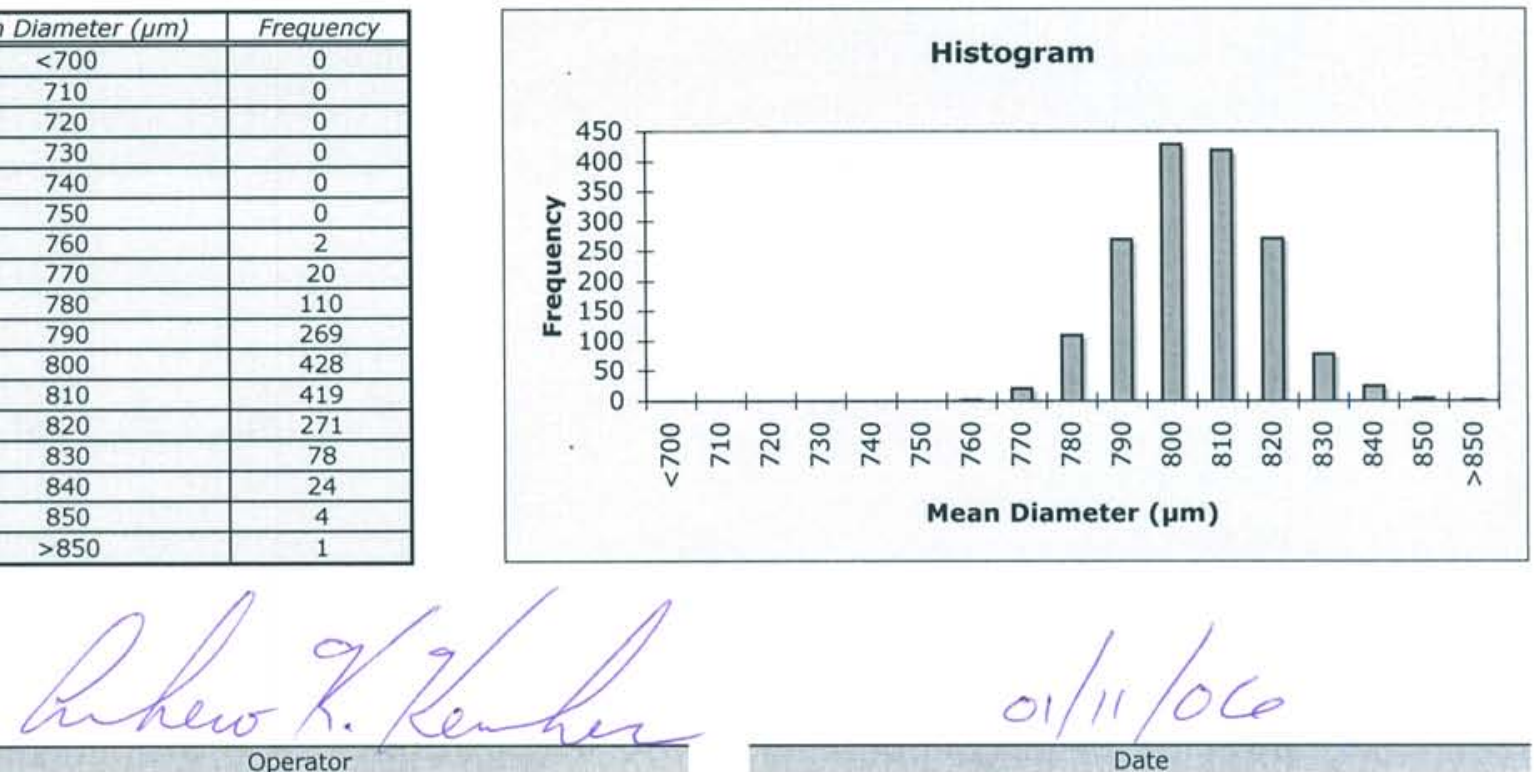

Operator 
ORNL/TM-2006/019, Rev. 1

Data Report Form DRF-31: Measurement of Open Porosity using a Mercury Porosimeter

\begin{tabular}{|c|c|}
\hline Procedure: & AGR-CHAR-DAM-31 Rev. 0 \\
\hline Operator: & S. D. Nunn \\
\hline Coated particle batch ID: & LEU01-46T-J01 + part LEU01-46T-K01 \\
\hline Batch Description: & Baseline composite TRISO on BWXT kernel composite 69302 \\
\hline Thermocouple Expiration Date: & $5 / 23 / 06$ \\
\hline Penetrometer Expiration Date: & $5 / 25 / 06$ \\
\hline Completed DRF Filename: & IImc-agr|AGR\Porosimeter|S06011002\S06011002_DRF31R0.xIs \\
\hline
\end{tabular}

Mean average weight/particle $(g): 7.27 \mathrm{E}-04$

Uncertainty in mean average weight/particle $(g): 4.25 \mathrm{E}-07$

\begin{tabular}{|r|l|}
\hline Weight of particles $(\mathrm{g}):$ & 3.5018 \\
\hline Approximate number of particles: & 4817 \\
\hline Uncertainty in number of particles: & 3 \\
\hline Total envelope volume of sample $(\mathrm{cc}):$ & 1.276 \\
\hline Average envelope volume/particle $(\mathrm{cc}):$ & $2.65 \mathrm{E}-04$ \\
\hline Sample envelope density $(\mathrm{g} / \mathrm{cc}):$ & 2.745 \\
\hline
\end{tabular}

\begin{tabular}{|r|l|}
\hline Average particle diameter (microns): & $7.97 \mathrm{E}+02$ \\
\hline Average surface area/particle $(\mathrm{cm} 2):$ & $1.99 \mathrm{E}-02$ \\
\hline Total sample surface area $(\mathrm{cm} 2):$ & $9.61 \mathrm{E}+01$ \\
\hline Open porosity $(\mathrm{ml} / \mathrm{m} 2):$ & $1.19 \mathrm{E}-02$ \\
\hline Intruded mercury volume from $250-10000$ \\
\hline
\end{tabular}

Open porosity of OPyC.

Note: sample LEU01-46T-I01 was not used due to instrument malfunction during analysis.

\section{Comments}

Note: sample LEU01-46r-101 was not used due to instrument malfunction duning analysis.

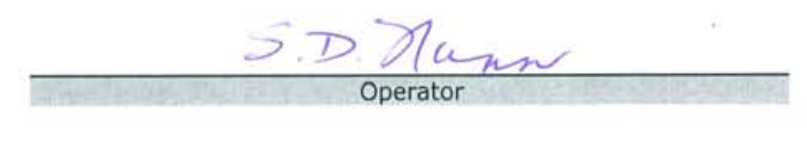

$\frac{1 / 10 / 06}{\text { Date }}$




\section{Supplement to DRF-21: Impurity Analysis Data}

\begin{tabular}{|c|c|}
\hline Procedure: & AGR-CHAR-DAM-21 Rev. 1 \\
\hline Operator: & Montgomery \\
\hline Sample ID: & LEU01-46T-F01 \\
\hline Sample Description: & Baseline Composite: TRISO on BWXT kernel composite 69302 \\
\hline Filename: & 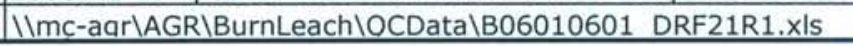 \\
\hline
\end{tabular}

\begin{tabular}{|c|c|c|c|c|}
\hline & Leach 1 & Leach 2 & Leach 3 & \\
\hline $\begin{array}{r}\text { Sample number: } \\
\text { RMAL Analysis Number: }\end{array}$ & $\begin{array}{c}\text { B0601060101 } \\
060223-019\end{array}$ & $\begin{array}{c}0601180102 \\
060223-021\end{array}$ & & Total $\mu g$ \\
\hline Fe content of leach solution $(\mu \mathrm{g})$ & 8.80 & 1.53 & & 10.33 \\
\hline Uncertainty in measured Fe content $(\mu \mathrm{g})$ & 0.88 & 0.31 & & 0.93 \\
\hline $\mathrm{Cr}$ content of leach solution $(\mu \mathrm{g})$ & 1.61 & $<0.45$ & & $<2.06$ \\
\hline Uncertainty in measured $\mathrm{Cr}$ content $(\mu \mathrm{g})$ & 0.32 & & & 0.32 \\
\hline Mn content of leach solution $(\mu \mathrm{g})$ & 0.22 & 0.21 & & 0.43 \\
\hline Uncertainty in measured $\mathrm{Mn}$ content $(\mu \mathrm{g})$ & 0.04 & 0.04 & & 0.06 \\
\hline Co content of leach solution $(\mu \mathrm{g})$ & 0.59 & 0.84 & & 1.43 \\
\hline Uncertainty in measured Co content $(\mu \mathrm{g})$ & 0.12 & 0.17 & & 0.21 \\
\hline Ni content of leach solution $(\mu \mathrm{g})$ & $<0.77$ & $<0.77$ & & $<1.54$ \\
\hline \multicolumn{5}{|l|}{ Uncertainty in measured $\mathrm{Ni}$ content $(\mu \mathrm{g})$} \\
\hline $\mathrm{Cr}+\mathrm{Mn}+\mathrm{Co}+\mathrm{Ni}(\mu \mathrm{g})$ & $<3.19$ & $<2.27$ & & $<5.46$ \\
\hline Uncertainty in $\mathrm{Cr}+\mathrm{Mn}+\mathrm{Co}+\mathrm{Ni}(\mu \mathrm{g})$ & 0.34 & 0.18 & & 0.39 \\
\hline Ca content of leach solution $(\mu \mathrm{g})$ & 19.00 & 3.41 & & 22.41 \\
\hline Uncertainty in measured $\mathrm{Ca}$ content $(\mu \mathrm{g})$ & 1.90 & 0.68 & & 2.02 \\
\hline Al content of leach solution $(\mu \mathrm{g})$ & $<2.11$ & 2.77 & & $<4.88$ \\
\hline Uncertainty in measured $\mathrm{Al}$ content $(\mu \mathrm{g})$ & & 0.55 & & 0.55 \\
\hline Ti content of leach solution $(\mu \mathrm{g})$ & $<0.50$ & $<0.50$ & & $<1.00$ \\
\hline \multicolumn{5}{|l|}{ Uncertainty in measured Ti content $(\mu \mathrm{g})$} \\
\hline$V$ content of leach solution $(\mu \mathrm{g})$ & $<0.65$ & 0.66 & & $<1.31$ \\
\hline Uncertainty in measured V content $(\mu \mathrm{g})$ & & 0.13 & & 0.13 \\
\hline $\mathrm{Ti}+\mathrm{V}$ content of leach solution $(\mu \mathrm{g})$ & $<1.15$ & $<1.16$ & & $<2.31$ \\
\hline Uncertainty in measured $\mathrm{Ti}+\mathrm{V}$ content $(\mu \mathrm{g})$ & & 0.13 & & 0.13 \\
\hline
\end{tabular}

\section{Comments}

\section{Fued c. Muntfomery}

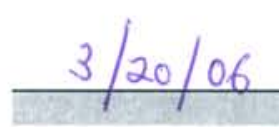

Date 


\section{Supplement to DRF-21: Impurity Analysis Data}

\begin{tabular}{|r|l|}
\hline Procedure: & AGR-CHAR-DAM-21 Rev. 1 \\
\hline Operator: & Montgomery \\
\hline Sample ID: & LEU01-46T-G01 \\
\hline Sample Description: & Baseline Composite: TRISO on BWXT kernel composite 69302 \\
\hline Filename: & IImc-aar\AGR\BurnLeach $\backslash$ OCData\B06010602 DRF21R1.xIs \\
\hline
\end{tabular}

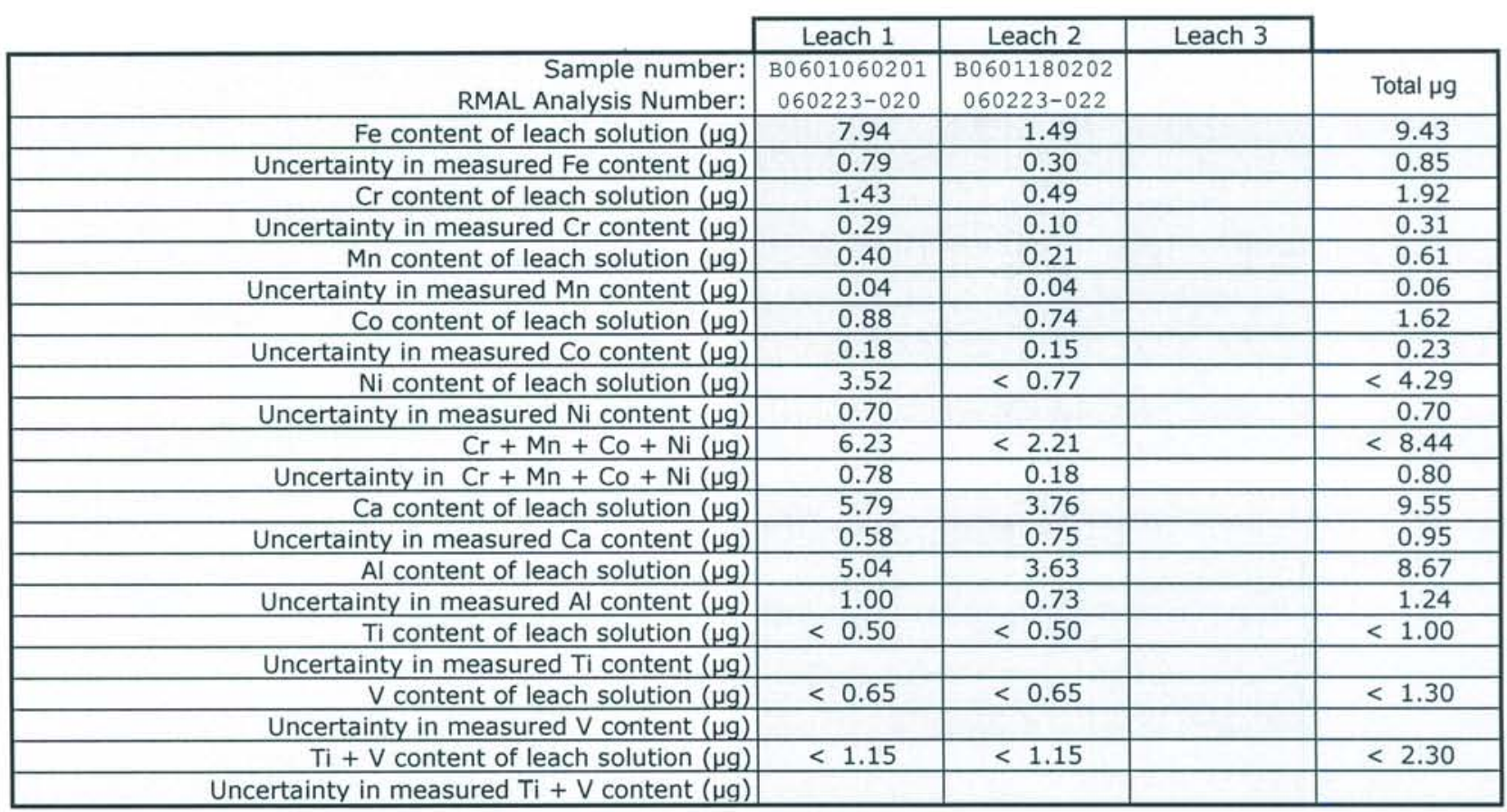

\section{Comments}




\section{Superceded data}

This section contains data related to the LEU01-46T TRISO-coated particle composite that has been superceded by more recent analysis.

It was determined that the average kernel weight as measured using procedure AGR-CHARDAM-22 Rev. 0 was of insufficient accuracy. The average kernel weight was remeasured to improve the accuracy of the reported value according to AGR-CHAR-DAM-22 Rev. 1. The measured value of the LEUCO average kernel weight changed from $(2.47 \pm 0.01) \cdot 10^{-4}$ grams per kernel to $(2.418 \pm 0.006) \cdot 10^{-4}$ grams per kernel. The measured value of the NUCO average kernel weight changed from $(2.39 \pm 0.03) \cdot 10^{-4}$ grams per kernel to $(2.308 \pm 0.009) \cdot 10^{-4}$ grams per kernel. On data report form DRF-15 for the measurement of average kernel envelope density, the average kernel weight is used to approximate the number of kernels in a sample from the weight of the sample. The approximate number of kernels in a sample is divided into the sample envelope volume to determine the average kernel envelope volume. The change in average kernel weight resulted in a significant change in the calculated average kernel envelope volume. On data report form DRF-16 for the buffer envelope density, the average kernel weight and volume are used to calculate the buffer density. The change in average kernel weight and volume resulted in a significant change in the calculated buffer densities previously used to determine acceptability of the buffer deposition conditions. The data in this compilation shows the new average kernel weights and average kernel volumes in sections 6 and 7, and the new buffer densities in section 8. Records showing the original values are included in this section. The new values for buffer density were about $10 \%$ higher than the originally reported values, but both were within the range specified in INL EDF-4380, Rev. 6.

In addition, the coating thicknesses on each TRISO-coated particle batch were remeasured after coated particle composite characterization was underway in order to improve the accuracy of the reported values. The original images of the particle cross sections were reanalyzed using an updated version of the image analysis program. This new version utilized a more robust algorithm for identifying the outer boundary of the outer pyrocarbon layer (OPyC). The result of this reanalysis was an increase of about $2 \mu \mathrm{m}$ in the measured value for the OPyC thickness on each particle. A small change in the measured thicknesses of the other layers also occurred due to the use of the outer OPyC boundary in the correction to the measured thickness to account for imaging off mid-plane (as described in AGR-CHAR-DAM-11R2). The data in this compilation shows the new coating thickness values in sections $11-14$. Records of the original measured values, which were initially used to determine the acceptance of each TRISO-coated particle batch for inclusion in the composite, are included in this section. Both the old and new coating thickness values for each batch were within the range specified in INL EDF-4380, Rev. 6. 


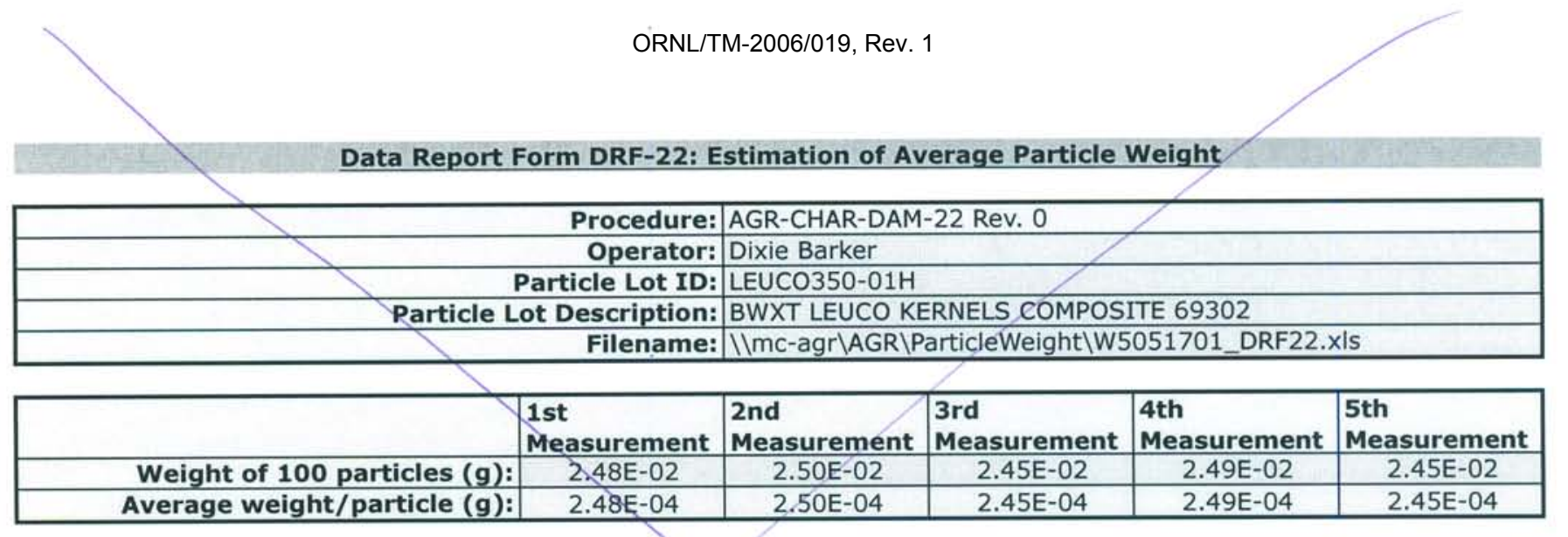

\begin{tabular}{cc|c} 
Mean average weight/particle $(\mathrm{g}):$ & $2.47 \mathrm{E}-04$ \\
mean average weight/particle $(\mathrm{g}):$ & $1.03 \mathrm{E}-06$
\end{tabular}
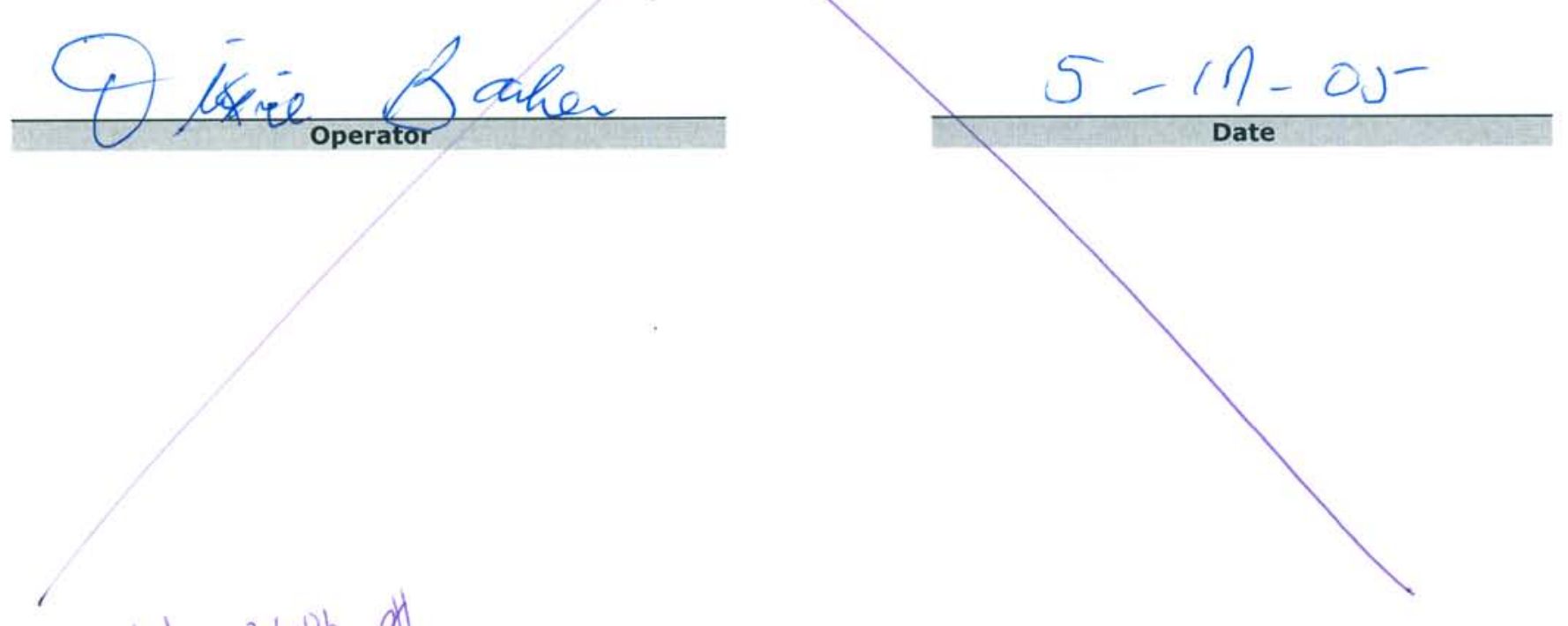

206 
Data Report Form DRF-15: Measurement of Average Kernel Envelope Density using a Mercury Porosimeter

\begin{tabular}{|r|l|}
\hline Procedure: & AGR-CHAR-DAM-15 Rev. 2 \\
\hline Operator: & S. D. NUNN \\
\hline Kernel Lot ID: & LEUCO350-01 \\
\hline Kernel Lot Description: & BWXT LEUCO KERNEL COMPOSITE 69302 \\
\hline Penetrometer Expiration Date: & $5 / 25 / 06$ \\
\hline Completed DRF Filename: & IImc-agr|AGRIPorosimeter|S05052701/S05052701_DRF15R2 \\
\hline
\end{tabular}

\begin{tabular}{|c|c|c|c|c|c|}
\hline \multicolumn{6}{|c|}{\begin{tabular}{r|c|c|} 
Mean average weight/kernel $(g):$ & $2.47 \mathrm{E}-04$ \\
\end{tabular}} \\
\hline \multicolumn{6}{|c|}{\begin{tabular}{|l|l|l} 
Uncertainty in mean average weight/kernel $(\mathrm{g}):$ & $1.03 \mathrm{E}-06$ \\
\end{tabular}} \\
\hline \\
\hline & Sample 1 & Sample 2 & Sample 3 & Sample 4 & Sample 5 \\
\hline Porosimeter data file number: & S05052701L & S05052702L & S05052703L & S05052704L & S05052705L \\
\hline Weight of kernels $(g)$ : & 12.5822 & 12.5075 & 12.8773 & 12.6448 & 12.6895 \\
\hline Approximate number of kernels: & 50940 & 50638 & 52135 & 51194 & 51374 \\
\hline Uncertainty in number of kernels: & 212 & 211 & 217 & 213 & 214 \\
\hline Envelope volume of sample (cc): & 1.150 & 1.145 & 1.180 & 1.157 & 1.164 \\
\hline Average envelope volume/kernel (cc): & $2.26 \mathrm{E}-0.5$ & $2,26 \mathrm{E}-05$ & $2.26 \mathrm{E}-05$ & $2.26 \mathrm{E}-05$ & $2.26 \mathrm{E}-05$ \\
\hline Sample envelope density $(\mathrm{g} / \mathrm{cc})$ : & 10.943 & 10.925 & 10.912 & 10.932 & 10.906 \\
\hline
\end{tabular}

\begin{tabular}{|r|l|}
\hline Mean average envelope volume/kernel $(\mathrm{cc}):$ & $2.261 \mathrm{E}-05$ \\
\hline Uncertainty in mean envelope volume/kernel (cc): & $1.4 \mathrm{E}-08$ \\
\hline Mean sample envelope density $(\mathrm{g} / \mathrm{cc}):$ & 10.924 \\
\hline Standard deviation in sample envelope density $(\mathrm{g} / \mathrm{cc}):$ & 0.015 \\
\hline
\end{tabular}
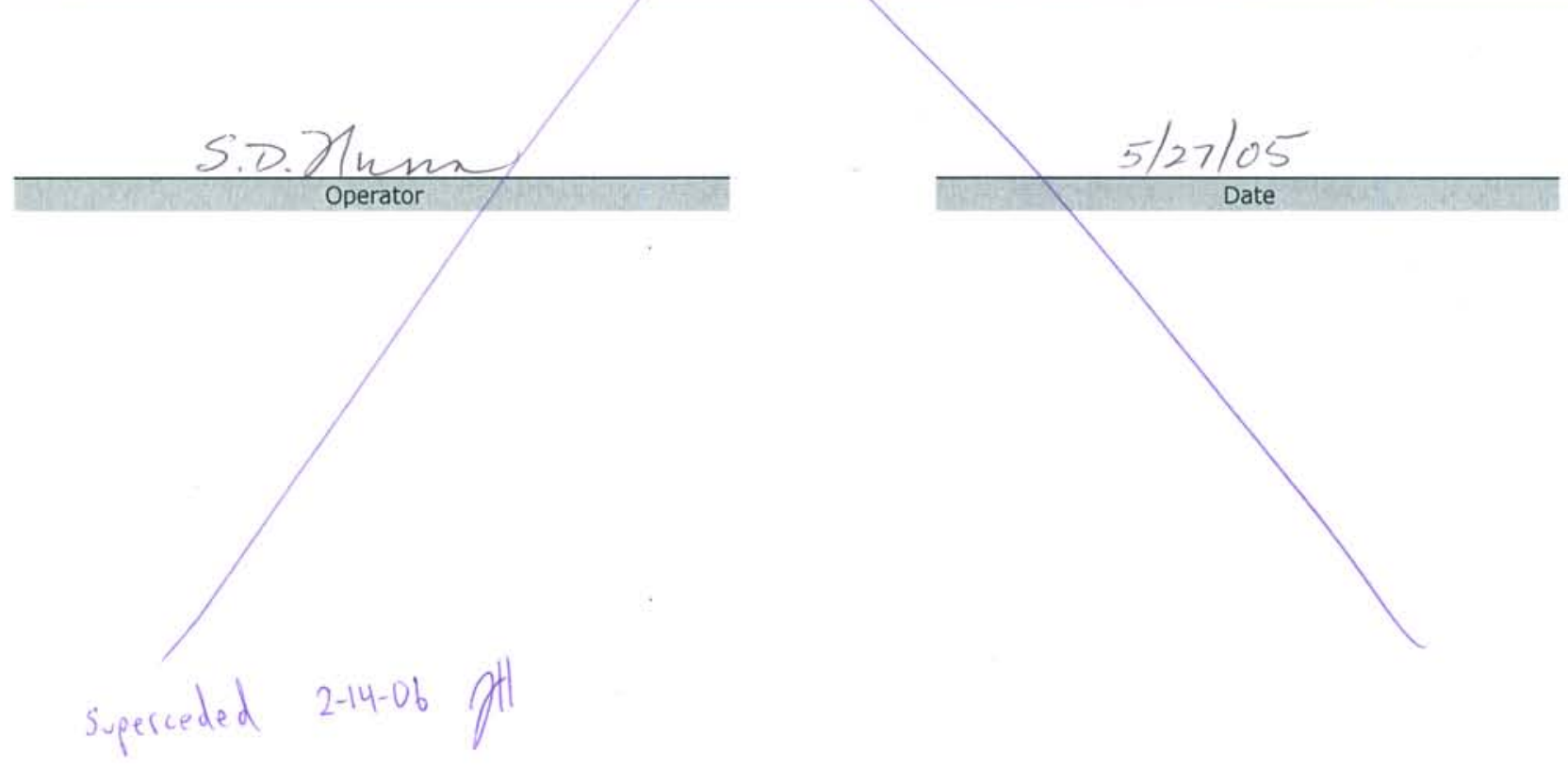
ORNL/TM-2006/019, Rev. 1

Data Report Form DRF-22: Estimation of Average Particle Weight

Procedure: AGR-CHAR-DAM-22 Rev. 0

Operator: Dixie Barker

Particle Lot ID: NUC0350-26-H

Particle Lot Description: BWXT NUCO Batch 69300

Filename: IImc-agr\AGR\ParticleWeight\W05052401_DRF22.xIs

\begin{tabular}{|c|l|l|l|l|c|}
\hline & $\begin{array}{l}\text { lIst } \\
\text { Measurement }\end{array}$ & $\begin{array}{l}\text { Ind } \\
\text { Measurement }\end{array}$ & $\begin{array}{l}\text { ard } \\
\text { Measurement }\end{array}$ & $\begin{array}{l}\text { 4th } \\
\text { Measurement }\end{array}$ & $\begin{array}{l}\text { Fth } \\
\text { Measurement }\end{array}$ \\
\hline Weight of 100 particles (g): & $2.31 \mathrm{E}-02$ & $2.38 \mathrm{E}-02$ & $2.44 \mathrm{E}-02$ & $2.45 \mathrm{E}-02$ & $2.38 \mathrm{E}-02$ \\
\hline Average weight/particle (g): & $2.31 \mathrm{E}-04$ & $2.38 \mathrm{E}-04$ & $2.44 \mathrm{E}-04$ & $2.45 \mathrm{E}-04$ & $2.38 \mathrm{E}-04$ \\
\hline
\end{tabular}

Mean average weight/particle (g): $2.39 \mathrm{E}-04$ Uncertainty in mean average weight/particle (g): $2.52 \mathrm{E}-06$
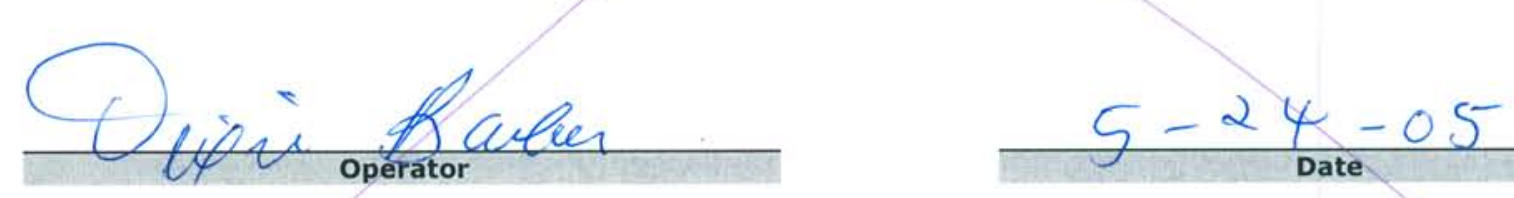

superseded 2-3-06 OH

208 
ORNL/TM-2006/019, Rev. 1

Data Report Form DRF-15: Measurement of Average Kernel Envelope Density using a Mercury Porosimeter

\begin{tabular}{|r|l|}
\hline Procedure: & AGR-CHAR-DAM-15 Rev. 2 \\
\hline Operator: & S. D. NUNN \\
\hline Kernel Lot ID: & NUCO350-26 \\
\hline Kernel Lot Description: & NUCO KERNEL COMPOSITE 69300 \\
\hline Thermocouple Expiration Date: & $5 / 23 / 06$ \\
\hline Penetrometer Expiration Date: & $5 / 25 / 06$ \\
\hline Completed DRF Filename: & IImc-agr\AGR\Porosimeter/S05052601/S05052601_DRF15R2 \\
\hline
\end{tabular}

\begin{tabular}{|c|c|c|c|c|c|}
\hline \multicolumn{6}{|c|}{\begin{tabular}{rl|l|} 
Mean average weight/kernel $(g):$ & $2.39 \mathrm{E}-04$ \\
\end{tabular}} \\
\hline \multicolumn{6}{|c|}{\begin{tabular}{|l|l|} 
Uncertainty in mean average weight/kernel $(\mathrm{g}):$ & $2.52 \mathrm{E}-06$ \\
\end{tabular}} \\
\hline & Sample 1 & Sample 2 & Sample 3 & Sample 4 & Sample 5 \\
\hline Porosimeter data file number: & S05052601L & S0502602L & S05052603L & S05052604L & S05052605L \\
\hline Weight of kernels $(g)$ : & 12.8272 & 12.9542 & 12.9410 & 12.9793 & 12.9624 \\
\hline Approximate number of kernels: & 53670 & 54202 & 54146 & 54307 & 54236 \\
\hline Uncertainty in number of kernels: & 566 & 571 & 571 & 573 & 572 \\
\hline Envelope volume of sample (cc): & 1.187 & 1.200 & 1.199 & 1.202 & 1.199 \\
\hline Average envelope volume/kernel (cc): & $2.21 \mathrm{E}-05$ & $2.21 \mathrm{E}-05$ & $2.21 \mathrm{E}-05$ & $2.21 \mathrm{E}-05$ & $2.21 \mathrm{E}-05$ \\
\hline Sample envelope density $(\mathrm{g} / \mathrm{cc}):$ & 10.805 & 10.792 & 10.797 & 10.799 & 10.807 \\
\hline
\end{tabular}

\begin{tabular}{|r|l|}
\hline Mean average envelope volume/kernel $(\mathrm{cc}):$ & $2.213 \mathrm{E}-05$ \\
\hline Uncertainty in mean envelope volume/kernel $(\mathrm{cc}):$ & $5.7 \mathrm{E}-09$ \\
\hline Mean sample envelope density $(\mathrm{g} / \mathrm{cc}):$ & 10.800 \\
\hline Standard deviation in sample envelope density $(\mathrm{g} / \mathrm{cc}):$ & 0.006 \\
\hline
\end{tabular}

S.DYMnn

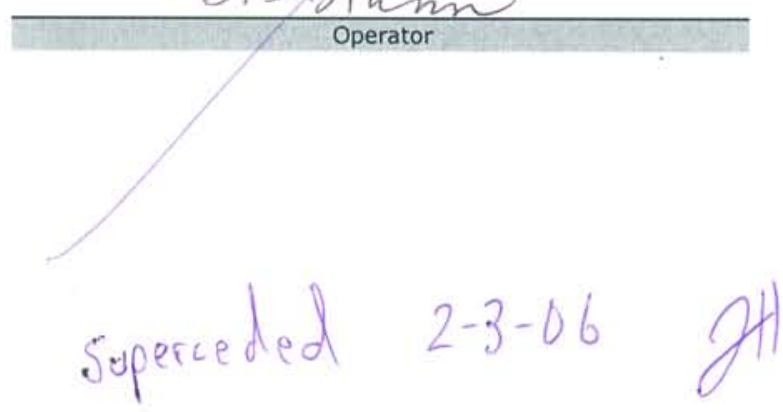

$5 / 26 / 05$

$\frac{2126 / 05}{\text { Date }}$




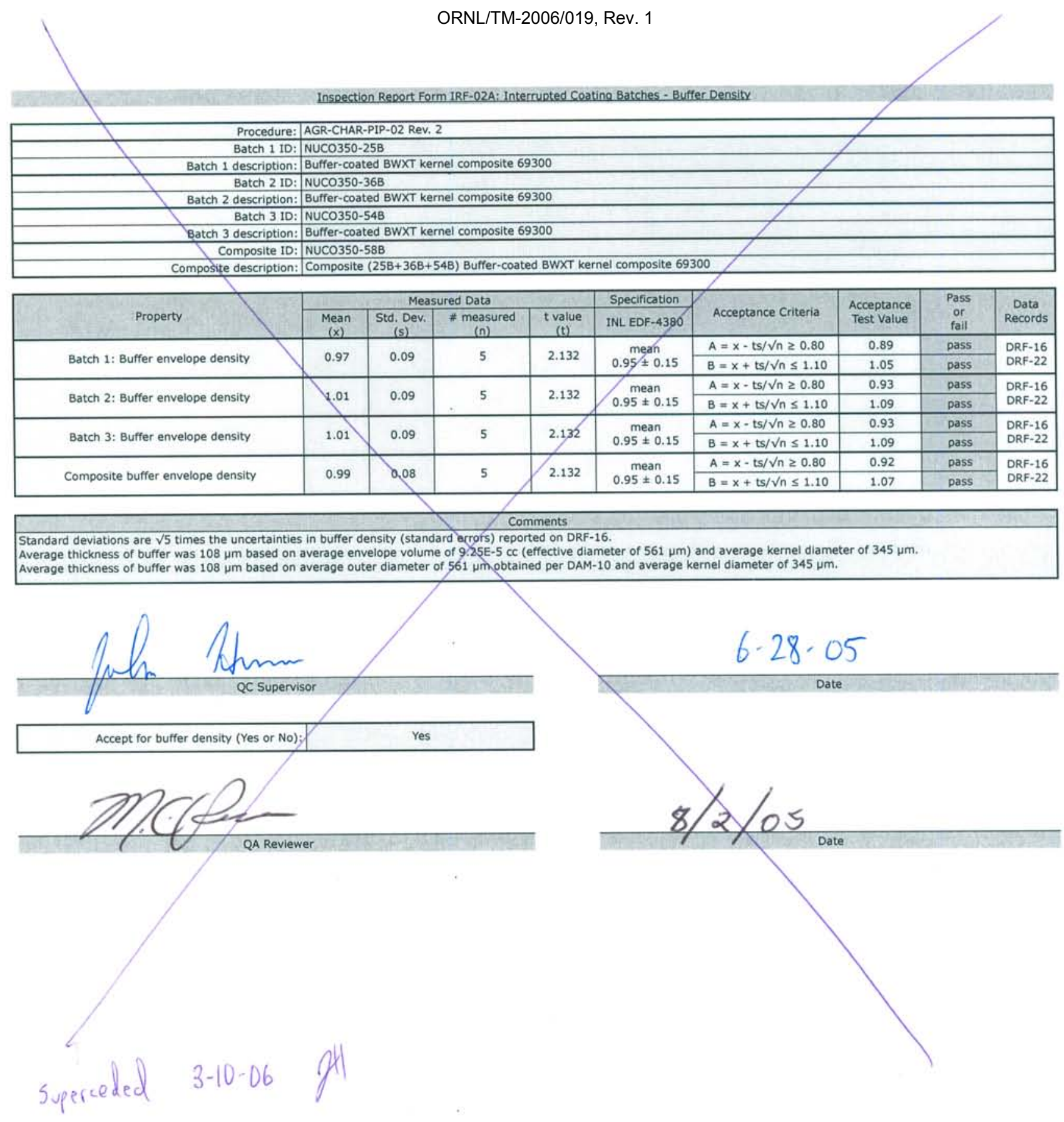




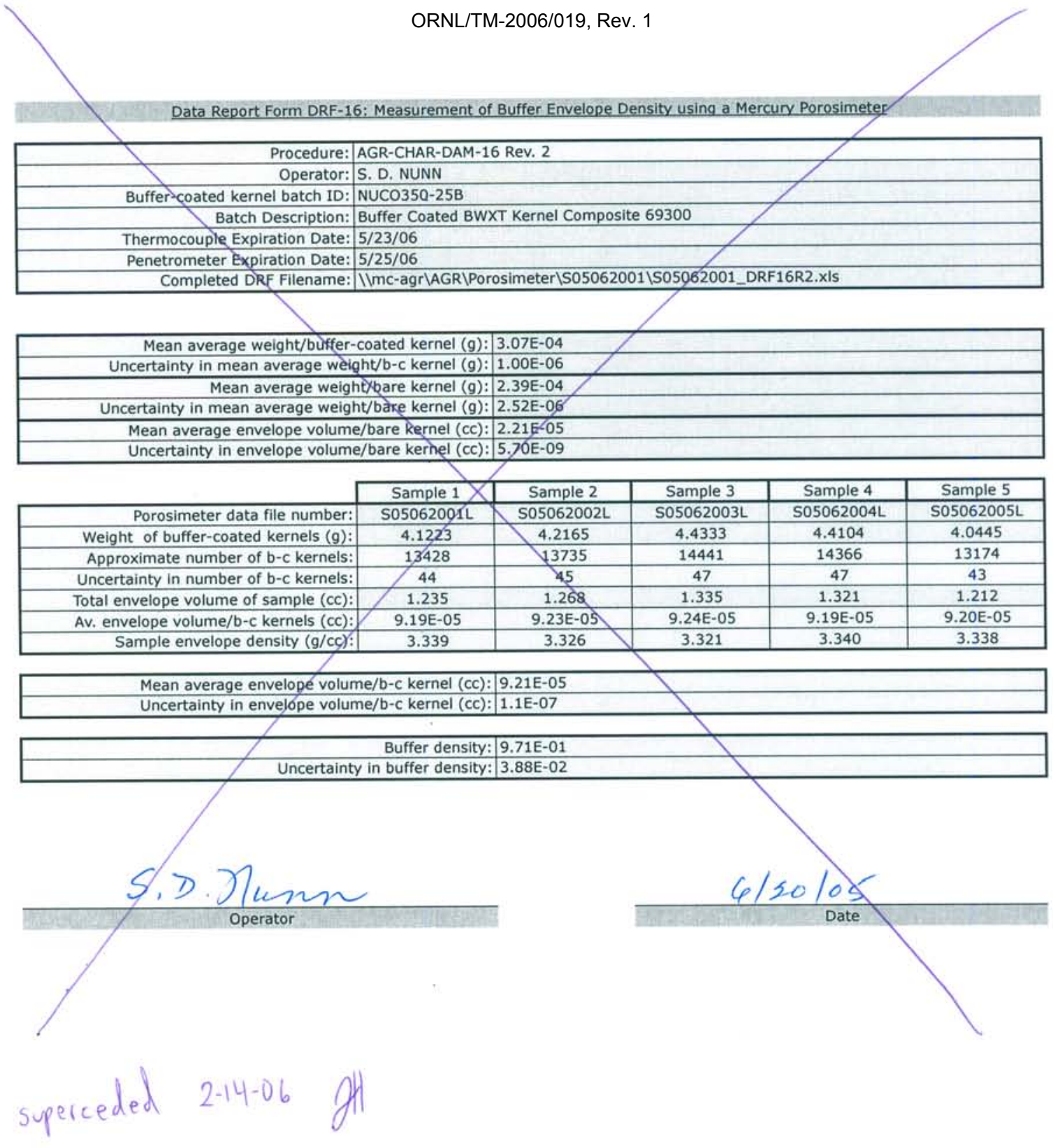




\section{ORNL/TM-2006/019, Rev. 1}

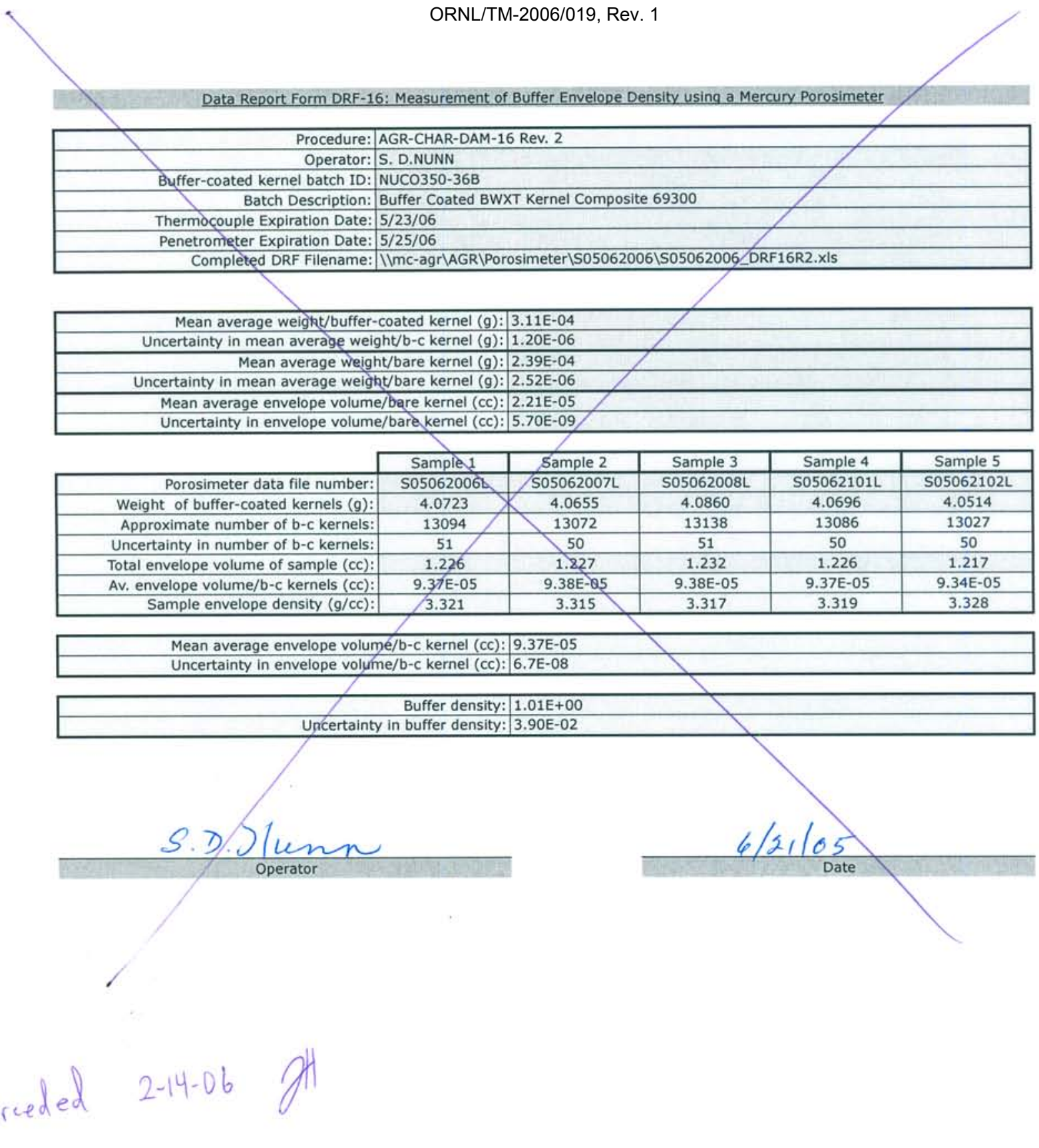


Data Report Form DRF-16: Measurement of Buffer Envelope Density using a Mercury Porosimeter

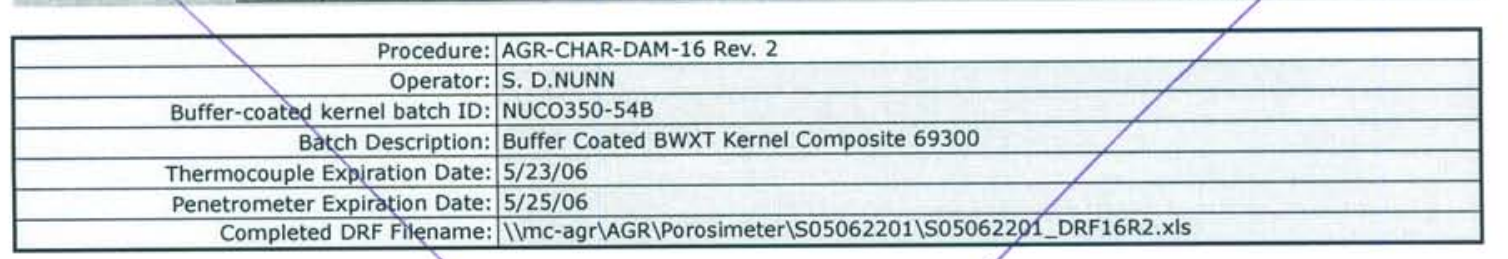

Completed DRF Filename: IImc-agr|AGR\Porosimeter|S05062201/S05062201_DRF16R2.xis

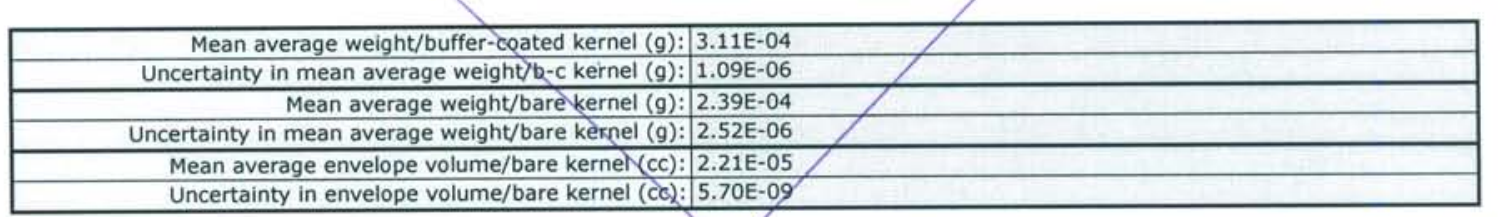

\begin{tabular}{|r|c|c|c|c|c|}
\cline { 2 - 5 } \multicolumn{1}{c|}{} & Sample 1 & S Sample 2 & Sample 3 & Sample 4 & Sample 5 \\
\hline Porosimeter data file number: & S05062201L & S05062202L & S05062203L & S05062204L & S05062205L \\
\hline Weight of buffer-coated kernels (g): & 4.1223 & 4.0694 & 4.0160 & 4.0391 & 4.0471 \\
\hline Approximate number of b-c kernels: & 13255 & 13085 & 12913 & 12987 & 13013 \\
\hline Uncertainty in number of b-c kernels: & 46 & 46 & 45 & 46 & 46 \\
\hline Total envelope volume of sample (cc): & $1 / 233$ & 1.225 & 1.209 & 1.215 & 1.215 \\
\hline Av. envelope volume/b-c kernels (cc): & $9.30 \mathrm{E}-05$ & $9.36 \mathrm{E}-05$ & $9.36 \mathrm{E}-05$ & $9.35 \mathrm{E}-05$ & $9.34 \mathrm{E}-05$ \\
\hline Sample envelope density (g/cc): & 3.344 & 3.323 & 3.323 & 3.326 & 3.331 \\
\hline
\end{tabular}

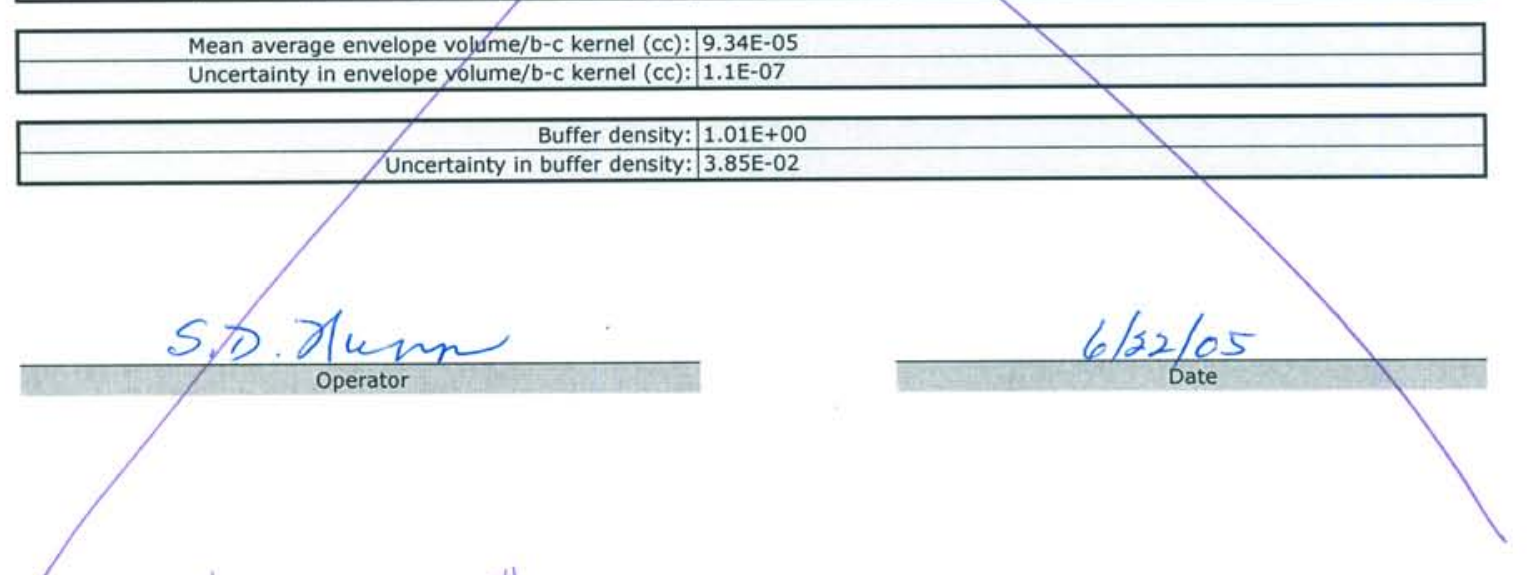

superceded 2-14-06 gtt 
Data Report Form DRF-16: Measurement of Buffer Envelope Density using a Mercury Porosimeter

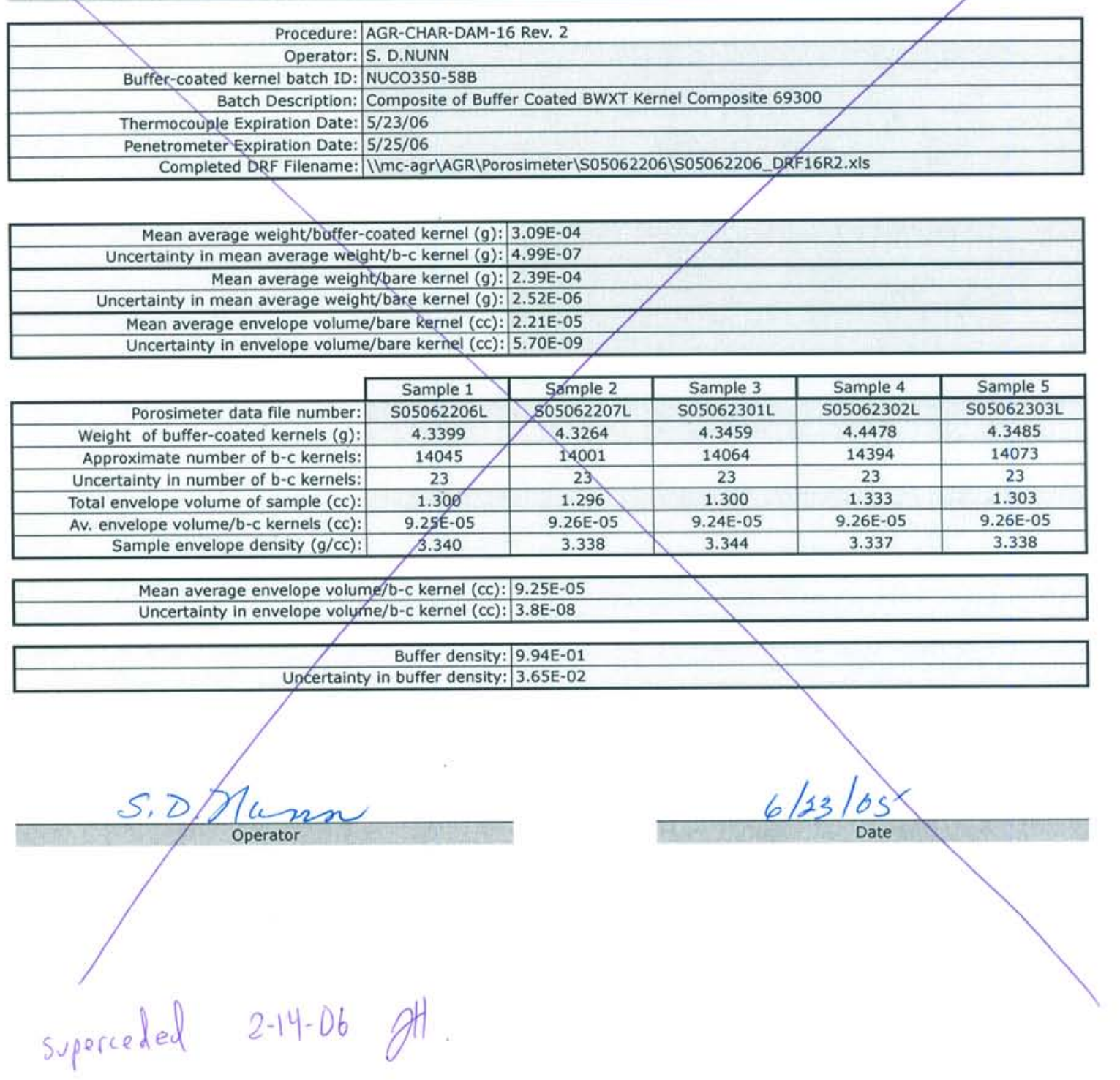


ORNL/TM-2006/019, Rev. 1

Data Report Form DRF-16: Measurement of Buffer Envelope Density using a Mercury Porosimeter

\begin{tabular}{|c|c|}
\hline Procedure: & AGR-CHAR-DAM-16 Rev. 2 \\
\hline Operator: & S. D. NUNN \\
\hline Buffer-coated kernel batch ID: & LEU01-16B \\
\hline Batch Description: & Buffer on BWXT LEUCO kernel composite 69302 \\
\hline Thermocouple Expiration Date: & $5 / 23 / 06$ \\
\hline Penetrometer Expiration Date: & $5 / 25 / 06$ \\
\hline Completed DRF Eilename: & IImc-agr|AGR\Porosimeter|S05110701/S05110701_DRF16R2.xls \\
\hline
\end{tabular}

Mean average weight/buffer-coated kernel $(g):$ : $3.20 \mathrm{E}-04$

\begin{tabular}{|c|c|}
\hline Mean average weight/buffer-coated kernel $(g)$ : & $3.20 \mathrm{E}-04$ \\
\hline Uncertainty in mean average weighteb-c kernel (g): & $8.22 \mathrm{E}-07$ \\
\hline Mean average weight/bare kernel $(\mathrm{g})$ : & $2.47 \mathrm{E}-04$ \\
\hline Uncertainty in mean average weight/bare kergel $(\mathrm{g})$ : & $1.03 \mathrm{E}-06$ \\
\hline Mean average envelope volume/bare kernel ( $(c c)$ : & $2.26 \mathrm{E}-0 \mathrm{~S}$ \\
\hline Uncertainty in envelope volume/bare kernel (cc)s & $1.40 E-08$ \\
\hline
\end{tabular}

\begin{tabular}{|r|c|c|c|c|c|}
\multicolumn{1}{c|}{} & \multicolumn{5}{c|}{} \\
\cline { 2 - 6 } \multicolumn{1}{c|}{} & Sample 1 & Sample 2 & Sample 3 & Sample 4 & Sample 5 \\
\hline Porosimeter data file number: & S05110701L & S05110702L & S05110703L & S05110704L & S05110705L \\
\hline Weight of buffer-coated kernels (g): & 4.0951 & 4.1007 & 4.2203 & 4.2527 & 4.3448 \\
\hline Approximate number of b-c kernels: & 12797 & 12815 & 13188 & 13290 & 13578 \\
\hline Uncertainty in number of b-c kernels: & 33 & 33 & 34 & 34 & 35 \\
\hline Total envelope volume of sample (cc): & 1.192 & 1.193 & 1.234 & 1.229 & 1.267 \\
\hline Av. envelope volume/b-c kernels (cc). & $9.32 \mathrm{E}-05$ & $9.31 \mathrm{E}-05$ & $9.36 \mathrm{E}-05$ & $9.25 \mathrm{E}-05$ & $9.33 \mathrm{E}-05$ \\
\hline Sample envelope density (g/cc): & 3.435 & 3.438 & 3.421 & 3.460 & 3.430 \\
\hline
\end{tabular}
Sample envelope density ( $\mathrm{g} / \mathrm{Cc}$ : 3.435 3.438 3.422

Mean average envelope volume/b-c kernel (cc): $9.31 \mathrm{E}-05$ Uncertainty in eqvelope volume/b-c kernel (cc): $1.8 \mathrm{E}-07$

\section{superceded $2-14-06$}

Buffer density: $1.04 \mathrm{E}+00$

S.D. D kn Operator 
ORNL/TM-2006/019, Rev. 1

Inspection Report Form IRF-03: Coated Particle Batches

Procedure: $\mid$ AGR-CHAR-PIP-03 Rev. 2

Coated particle batch ID: LEU01-21T

Coated particle batch description: TRISO on BWXT kernel composite 69302

\begin{tabular}{|c|c|c|c|c|c|c|c|c|c|}
\hline \multirow[b]{2}{*}{ Property } & \multicolumn{4}{|c|}{ Measured Data } & Specification & \multirow[b]{2}{*}{ Acceptance Criteria } & \multirow{2}{*}{$\begin{array}{l}\text { Acceptance } \\
\text { Test Value }\end{array}$} & \multirow{2}{*}{$\begin{array}{l}\text { Pass } \\
\text { or } \\
\text { fail }\end{array}$} & \multirow{2}{*}{$\begin{array}{c}\text { Data } \\
\text { Records }\end{array}$} \\
\hline & $\begin{array}{c}\text { Mean } \\
(x)\end{array}$ & \begin{tabular}{|c|}
$\begin{array}{c}\text { Std. Dev. } \\
\text { (s) }\end{array}$ \\
\end{tabular} & $\begin{array}{c}\text { \# measured } \\
(\mathrm{n})\end{array}$ & $\begin{array}{l}t \text { value } \\
(t)\end{array}$ & INL EDF- 4380 & & & & \\
\hline \multirow{2}{*}{$\begin{array}{l}\text { Average buffer thickness for } \\
\text { each particle }(\mu \mathrm{m})\end{array}$} & \multirow{2}{*}{101.2} & \multirow{2}{*}{7.8} & \multirow{2}{*}{106} & \multirow{2}{*}{1.659} & \multirow{2}{*}{$\begin{array}{c}\text { mean } \\
100 \pm 15\end{array}$} & A. $f x-t s / \sqrt{ } n \geq 85$ & 99.9 & pass & \multirow{2}{*}{$\begin{array}{l}\text { DRF-08 } \\
\text { DRF-11 }\end{array}$} \\
\hline & & & & & & $B=x+t s / \sqrt{ } n \leq 115$ & 102.5 & pass & \\
\hline \multirow{2}{*}{$\begin{array}{l}\text { Average IPyC thickness for } \\
\text { each particle }(\mu \mathrm{m})\end{array}$} & \multirow{2}{*}{40.1} & \multirow{2}{*}{2.0} & \multirow{2}{*}{157} & \multirow{2}{*}{1.655} & \multirow{2}{*}{$\begin{array}{l}\text { mean } \\
40 \pm 5\end{array}$} & $A=x-t s / \sqrt{n} \geq 35$ & 39.8 & pass & \multirow{2}{*}{$\begin{array}{l}\text { DRF-08 } \\
\text { DRF-11 }\end{array}$} \\
\hline & & & & & & $B=x+t s / \sqrt{ } n \leq 45$ & 40.4 & pass & \\
\hline \multirow{2}{*}{$\begin{array}{l}\text { Average SiC thickness for } \\
\text { each particle }(\mu \mathrm{m})\end{array}$} & \multirow{2}{*}{37.6} & \multirow{2}{*}{0.9} & \multirow{2}{*}{157} & \multirow{2}{*}{1.655} & \multirow{2}{*}{ mean } & $A=x-t s / \sqrt{ } n \geq 31$ & 37.5 & pass & \multirow{2}{*}{$\begin{array}{l}\text { DRF-08 } \\
\text { DRF-11 }\end{array}$} \\
\hline & & & & & & $B=x+t s / \sqrt{n} \leq 39$ & 37.7 & pass & \\
\hline \multirow{2}{*}{$\begin{array}{l}\text { Average OPyC thickness for } \\
\text { each particle }(\mu \mathrm{m})\end{array}$} & \multirow[t]{2}{*}{40.9} & \multirow[t]{2}{*}{2.3} & \multirow[t]{2}{*}{157} & \multirow{2}{*}{1.655} & \multirow{2}{*}{$\begin{array}{l}\text { mean } \\
40 \pm 5\end{array}$} & $A=x-t s / \sqrt{n} \geq 35$ & 40.6 & pass & \multirow{2}{*}{$\begin{array}{l}\text { DRF-08 } \\
\text { DRF-11 }\end{array}$} \\
\hline & & & & & & $B=x+t s / \sqrt{n} \leq 45$ & 41.2 & pass & \\
\hline Particles with missing OPyC & & & 15578 & & $\begin{array}{l}\text { defect fraction } \\
\leq 6.0 \times 10^{-4}\end{array}$ & $\leq 4$ in 15,500 & 0 & pass & DRF-19 \\
\hline
\end{tabular}

Comments

Goldspot analysis performed on missing OPyC sample for information only. 10 gurt of 15578 gold spots observed.
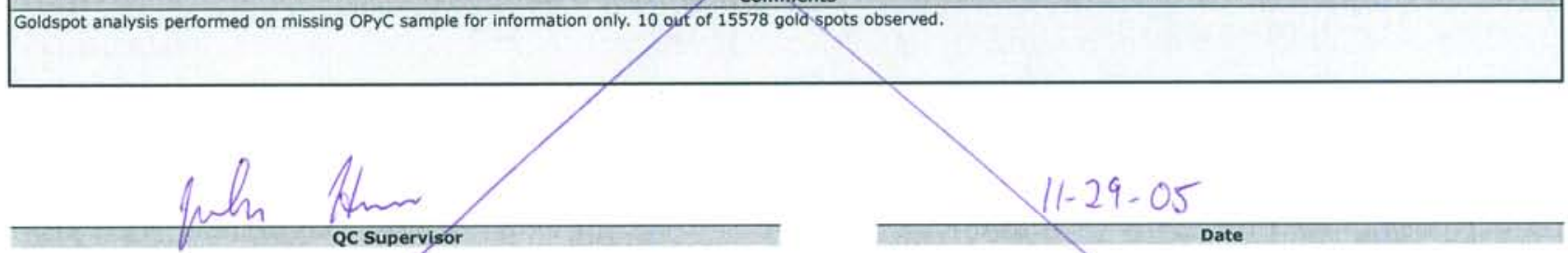

Yes

superceded 3.23-06 gll 


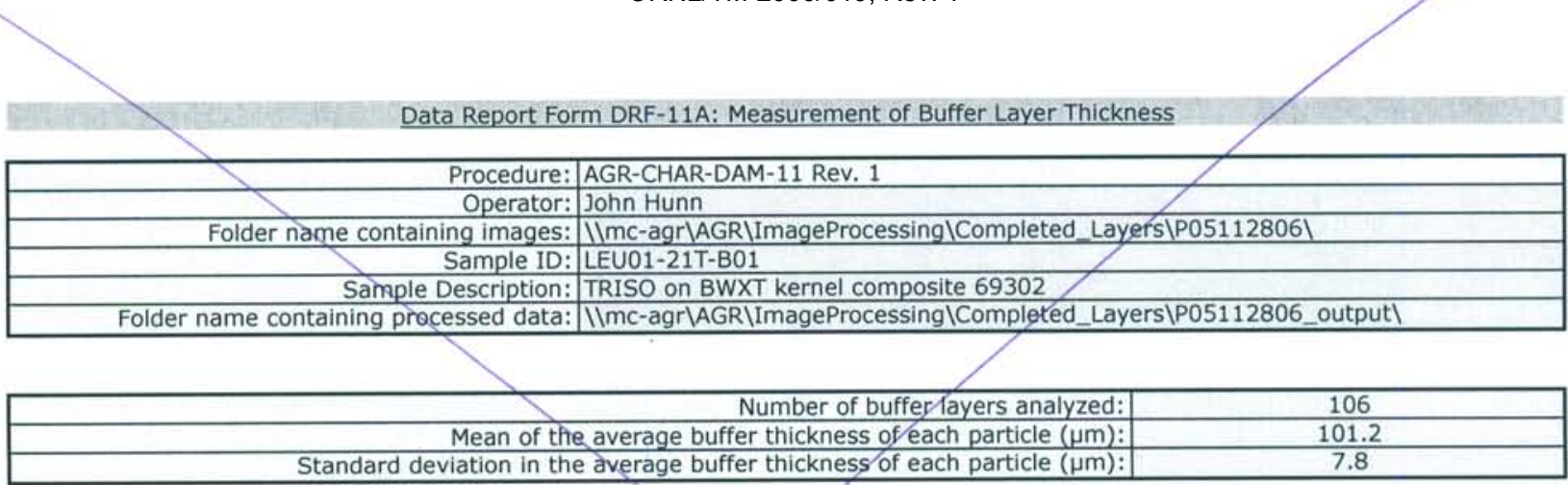

Distribution of the average buffer layer thickness (top binned)

\begin{tabular}{|c|c|}
\hline Buffer Thickness $(\mu \mathrm{m})$ & Frequency \\
\hline \hline$<55$ & 0 \\
\hline 60 & 0 \\
\hline 65 & 0 \\
\hline 70 & 0 \\
\hline 75 & 0 \\
\hline 80 & 0 \\
\hline 85 & 3 \\
\hline 90 & 8 \\
\hline 95 & 10 \\
\hline 100 & 23 \\
\hline 105 & 26 \\
\hline 110 & 22 \\
\hline 115 & 11 \\
\hline 120 & 3 \\
\hline 125 & 0 \\
\hline$>125$ & 0 \\
\hline & \\
\hline &
\end{tabular}

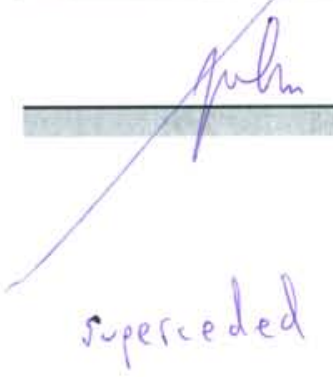

Thin Operator

$$
25
$$

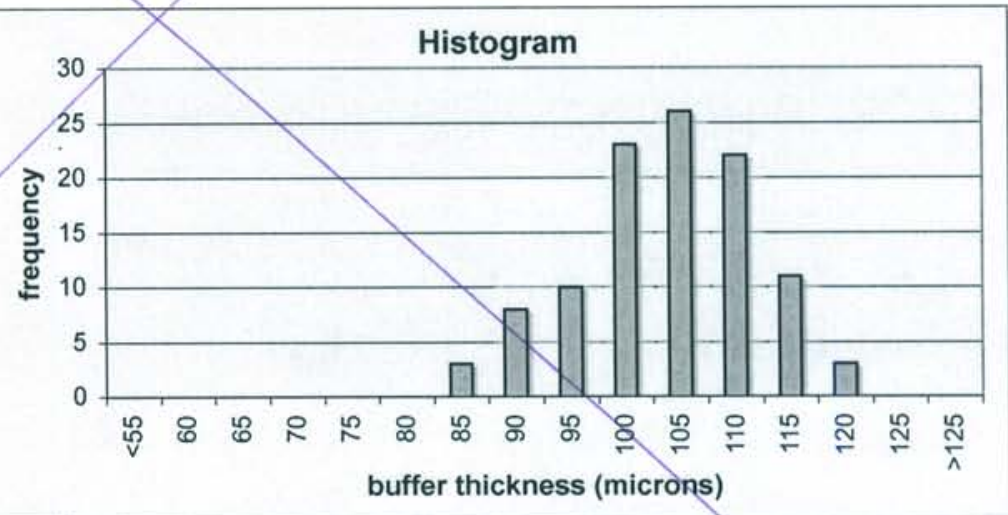

\section{$1-24-06 \quad \mathrm{yt}$}

\section{$11-29-05$}

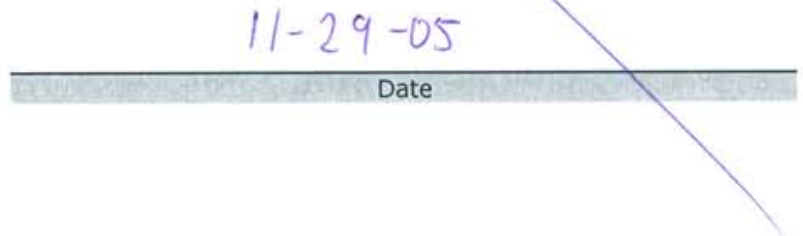


Data Report Form DRF-11B: Measurement of Inner Pyrocarbon Layer Thickness

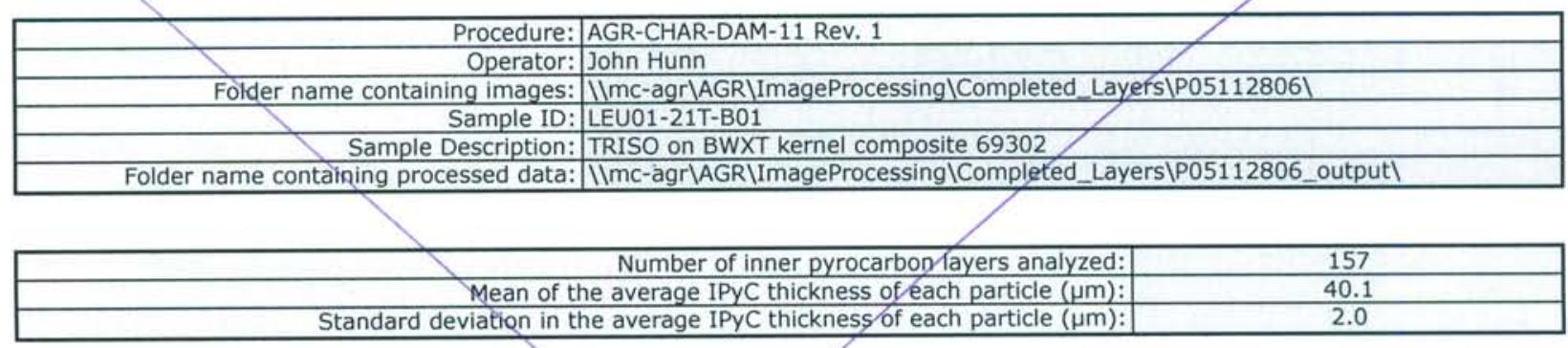
Standard deviation in the average IPyC thickness of each particle $(\mu \mathrm{m})$ :

\section{Distribution of the average IPyClayer thickness (top binned)}

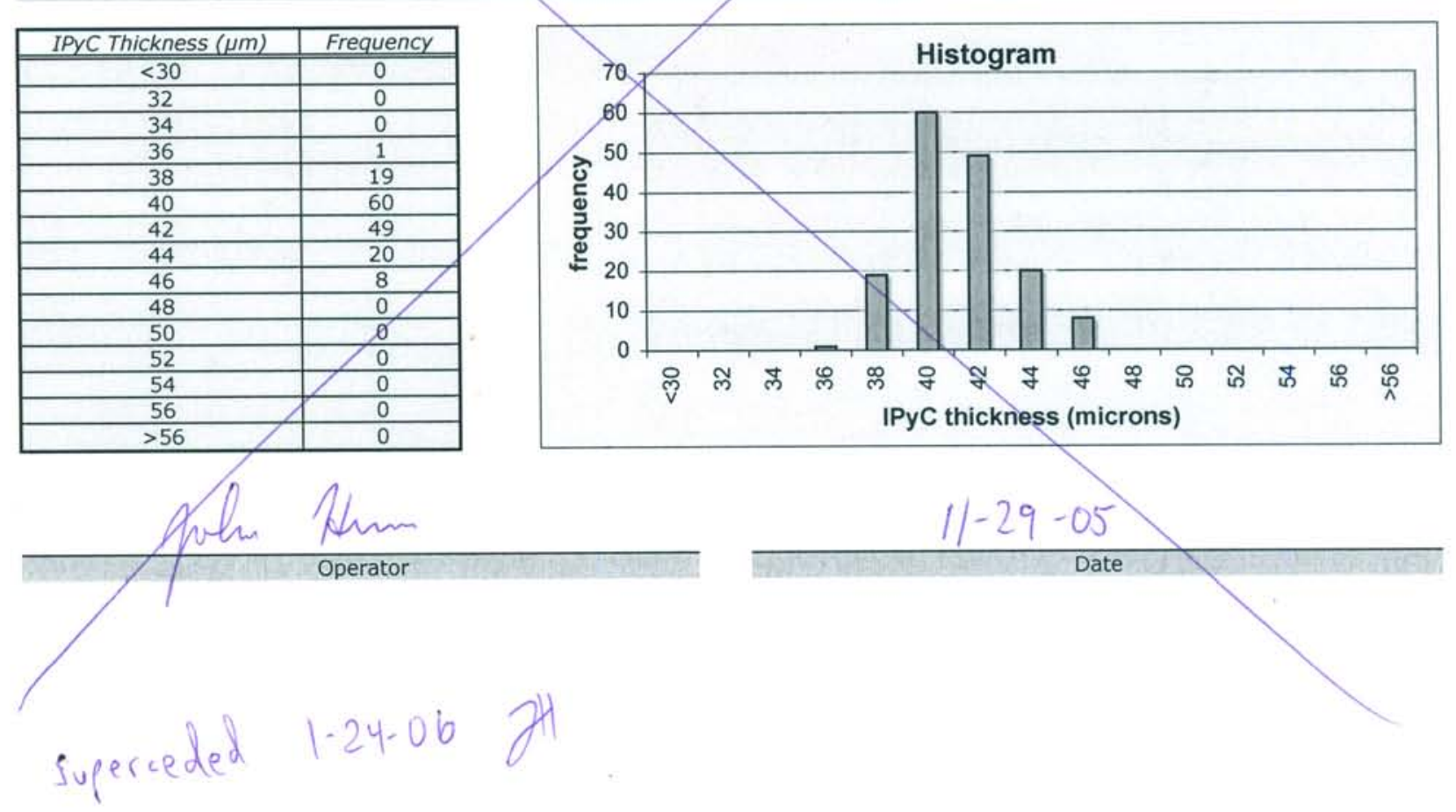




\begin{tabular}{|c|c|c|}
\hline 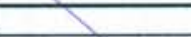 & Procedure: & AGR-CHAR-DAM-11 Rev. 1 \\
\hline 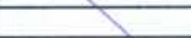 & Operator: & John Hunn \\
\hline ( & Folder name containing images: & 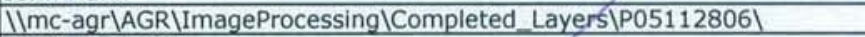 \\
\hline & Sample ID: & LEU01-21T-B01 \\
\hline & Sample Description: & TRISO on BWXT kernel composite 69302 \\
\hline
\end{tabular}

Folder name containing processed data: IImc-agr|AGR\ImageProcessing|Completed_Layers\P05112806_output|

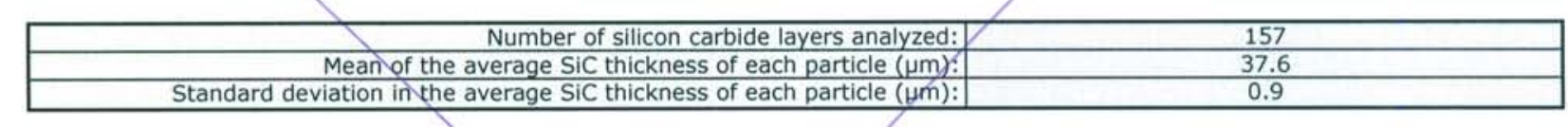

Distribution of the average SiC layer thickness (top binned)

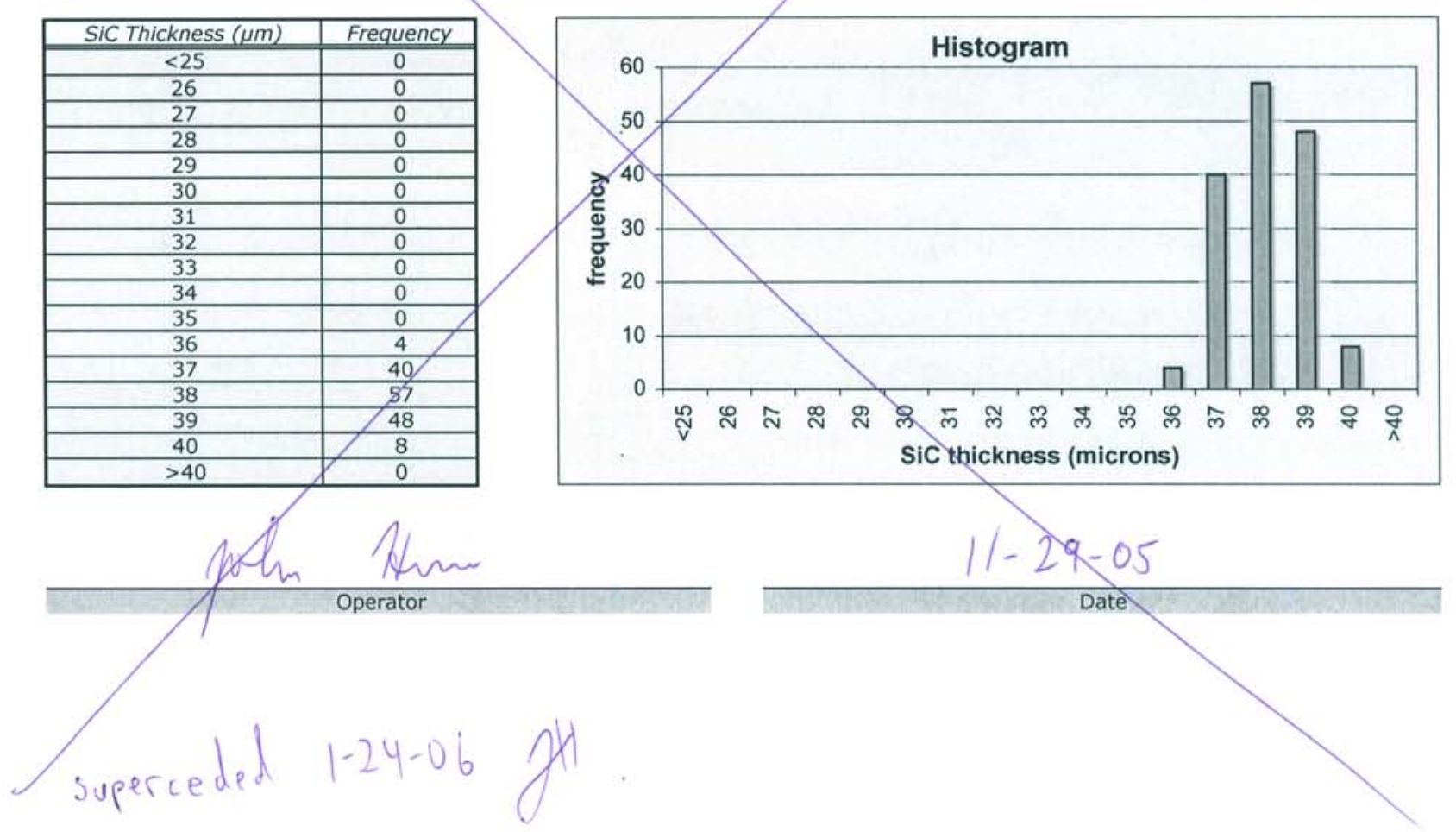


Data Report Form DRF-11D: Measurement of Outer Pyrocarbon Layer Thickness

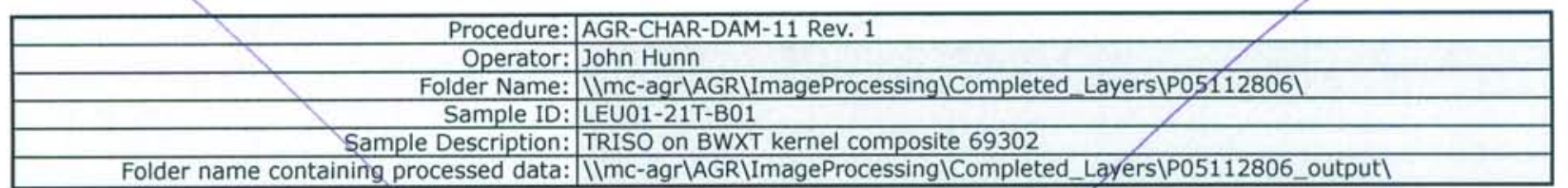

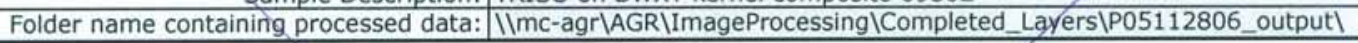

\begin{tabular}{|r|r|r|r|}
\hline Number of outer pyrocarbon layers analyzed: & \\
\hline Mean of the average OPyC thickness of each particle $(\mu \mathrm{m}):$ & 157 \\
\hline Standard deviation in the average OPyC thickness of each particle $(\mu \mathrm{m}):$ & 40.9 \\
\hline
\end{tabular}

Distribution of the average OPyC layer thickness (top binned)

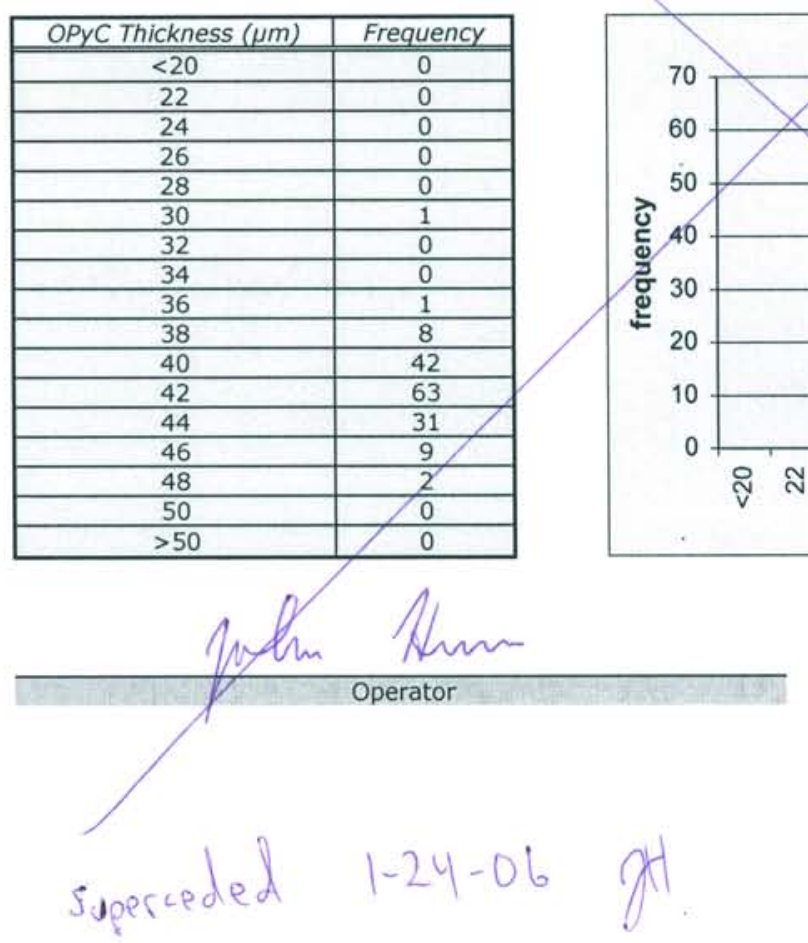

$$
11-29-05
$$

Date 
ORNL/TM-2006/019, Rev. 1

Inspection Rebort Form IRF-03: Coated Particle Batches

Coated particle batch ID: LEUO1-35T

Coated particle batch description: TRISO on BWXT kernel composite 69302

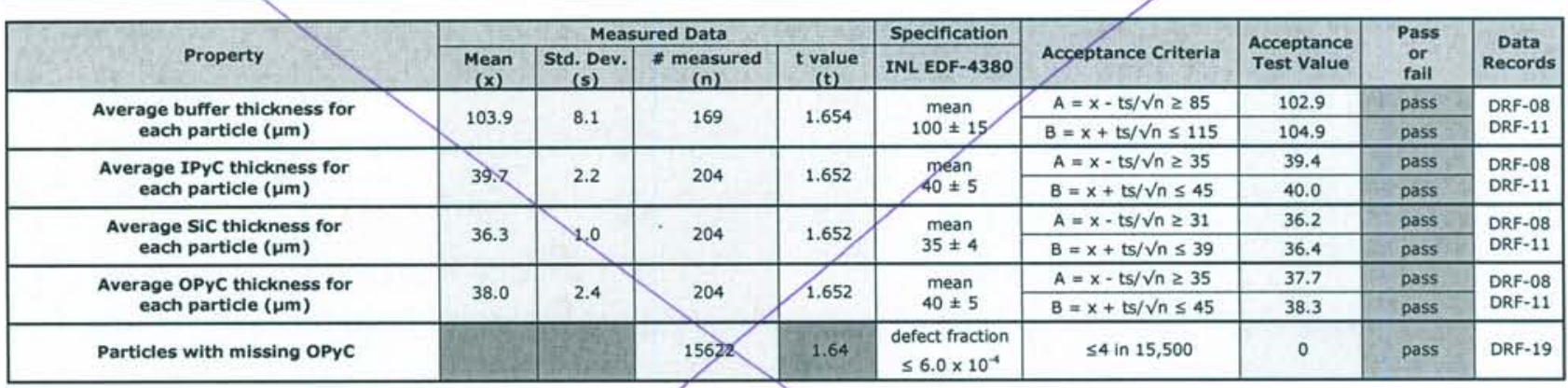

Comments

Goldspot analysis performed on missing OPyC sample for information oply. 17 out of 15622 gold spots observed.
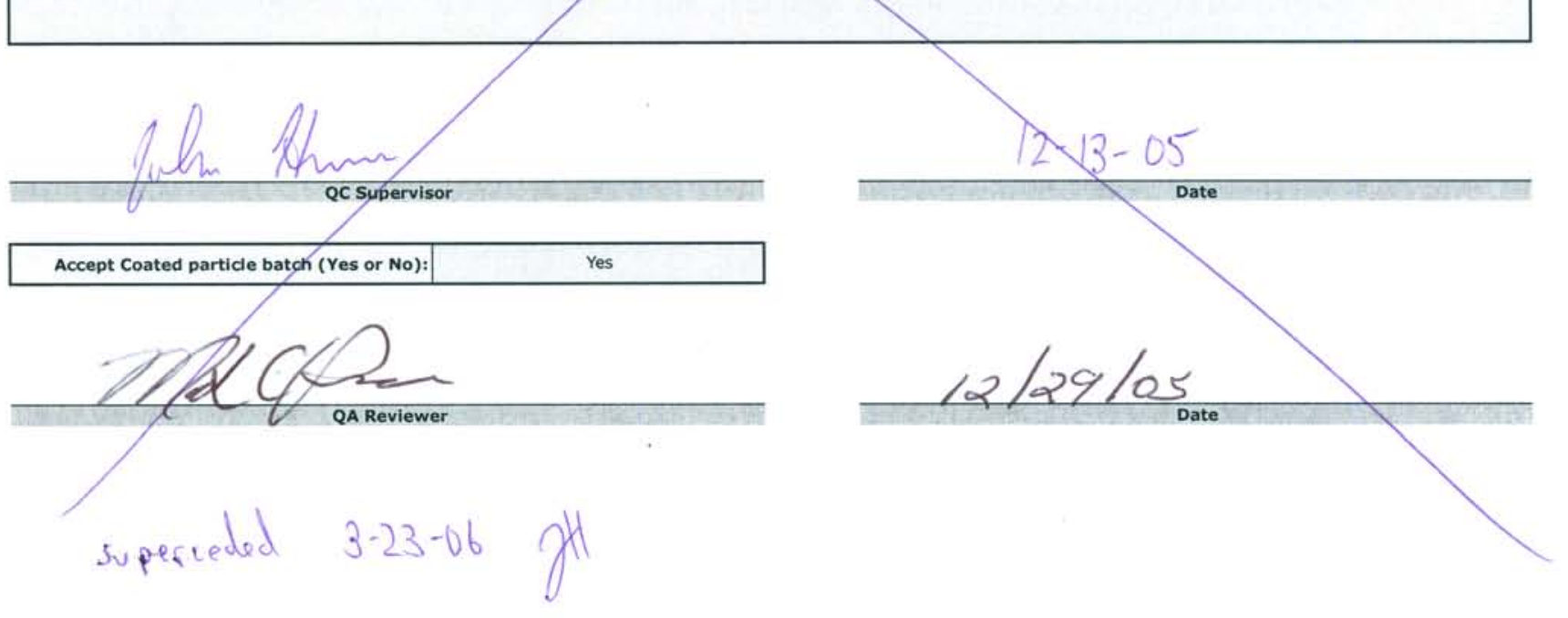


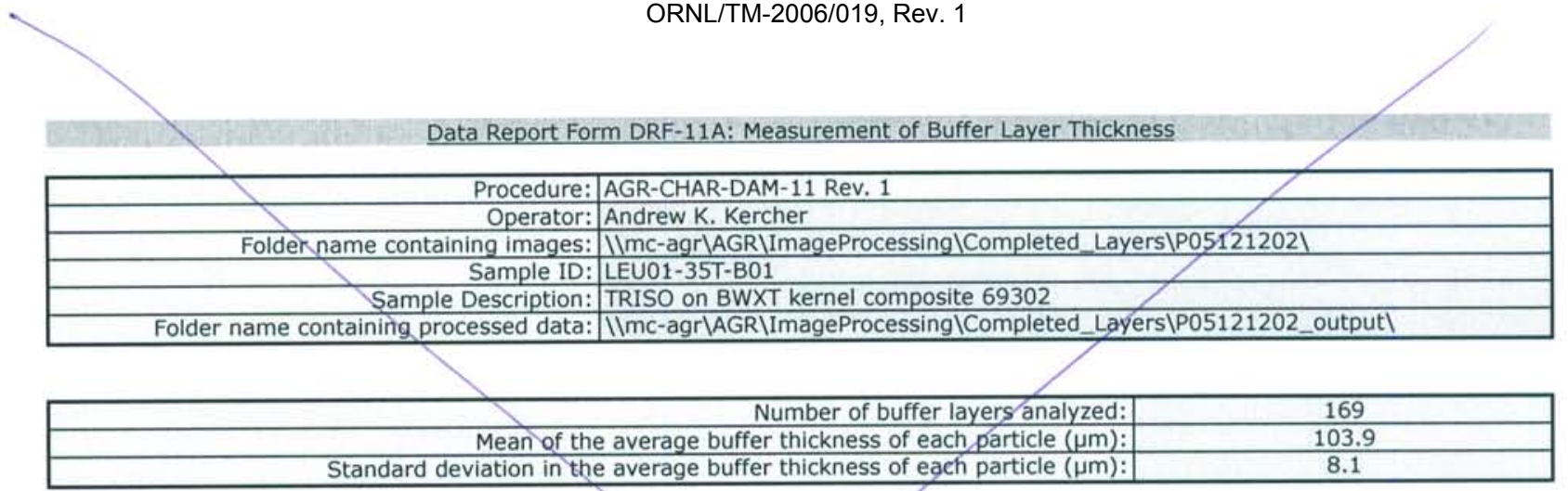
Standard deviation in the average buffer thickness of each particle $(\mu \mathrm{m})$ :

Distribution of the average buffer layer thickness (top binned)

\begin{tabular}{|c|c|}
\hline Buffer Thickness $(\mu \mathrm{m})$ & Frequency \\
\hline \hline$<55$ & 0 \\
\hline 60 & 0 \\
\hline 65 & 0 \\
\hline 70 & 0 \\
\hline 75 & 0 \\
\hline 80 & 0 \\
\hline 85 & 1 \\
\hline 90 & 4 \\
\hline 95 & 18 \\
\hline 100 & 34 \\
\hline 105 & 42 \\
\hline 110 & 31 \\
\hline 115 & 22 \\
\hline 120 & 10 \\
\hline 125 & 6 \\
\hline$>125$ & 1 \\
\hline
\end{tabular}

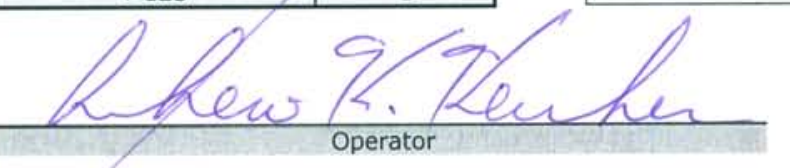

superceded $1-26-06$
Histogram

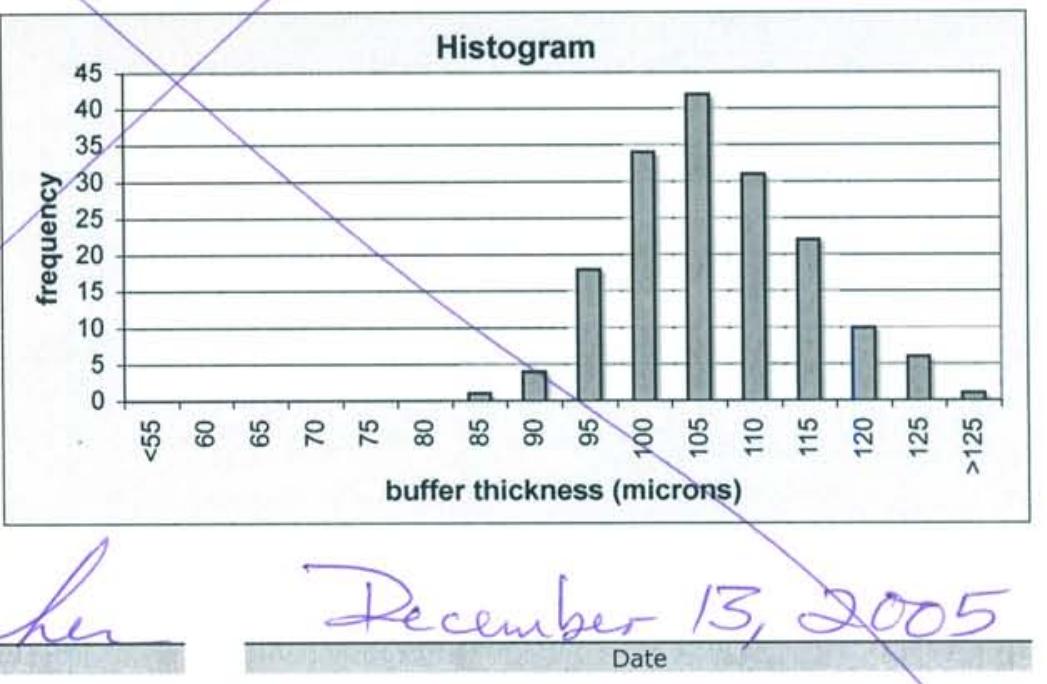


Data Report Form DRF-11B: Measurement of Inner Pyrocarbon Layer Thickness

\begin{tabular}{|c|c|}
\hline Procedure: [ & AGR-CHAR-DAM-11 Rev. 1 \\
\hline Operator: & Andrew K. Kercher \\
\hline Folder name containing images: & ||mc-agr|AGR|ImageProcessing|Completed_Layers|P05121202| \\
\hline Sample ID:| & LEU01-35T-B01 \\
\hline Sample Description: & TRISO on BWXT kernel composite 69302 \\
\hline Folder name containing processed data: & IImc-agr|AGR\ImageProcessing|Completed_Layers\P05121202_output\ \\
\hline & Number of inner pyrocarbon láyers analyzed: \\
\hline Mean of th & e average IPyC thickness of each particle $(\mu \mathrm{m})$ : \\
\hline Standard deviation in th & e average IPyC thickness of each particle $(\mu \mathrm{m}):$ \\
\hline
\end{tabular}

\section{Distribution of the average IPYC Jayer thickness (top binned)}

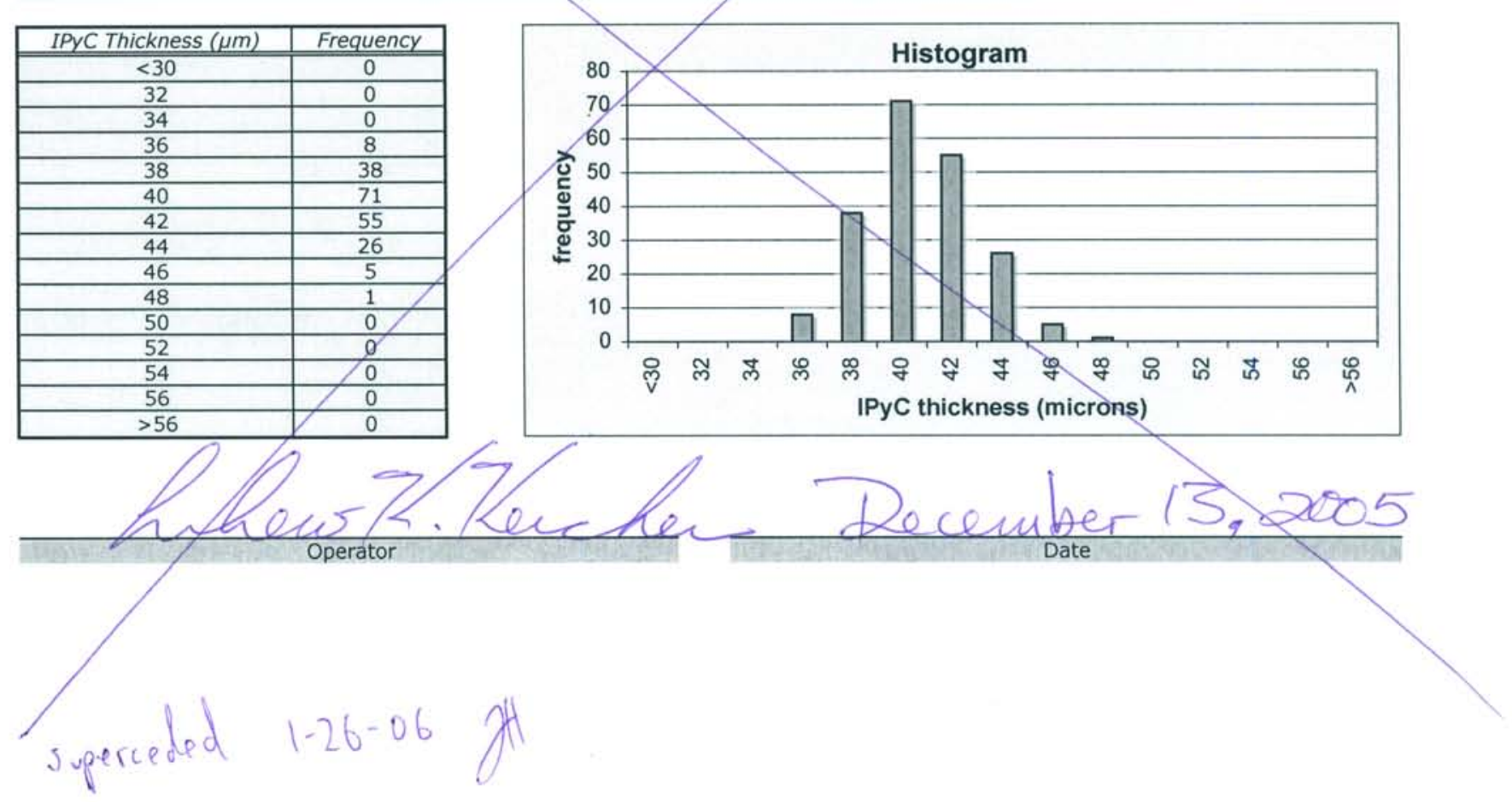




\section{Data Report Form DRF-11C: Measurement of Silicon Carbide Layer Thickness}

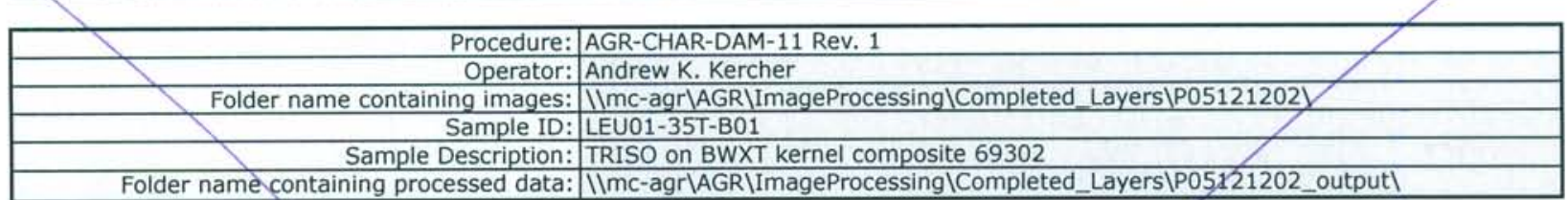

\begin{tabular}{|r|r|}
\hline Number of silicon carbide layers analyzed: & 204 \\
\hline Mean of the average SiC thickness of each particle $(\mu \mathrm{m}):$ & 36.3 \\
\hline Standard deviation in the average SiC thickness of each particle $(\mu \mathrm{m}):$ & 1.0 \\
\hline
\end{tabular}

\section{Distribution of the average SiC layer thickness (top binned)}

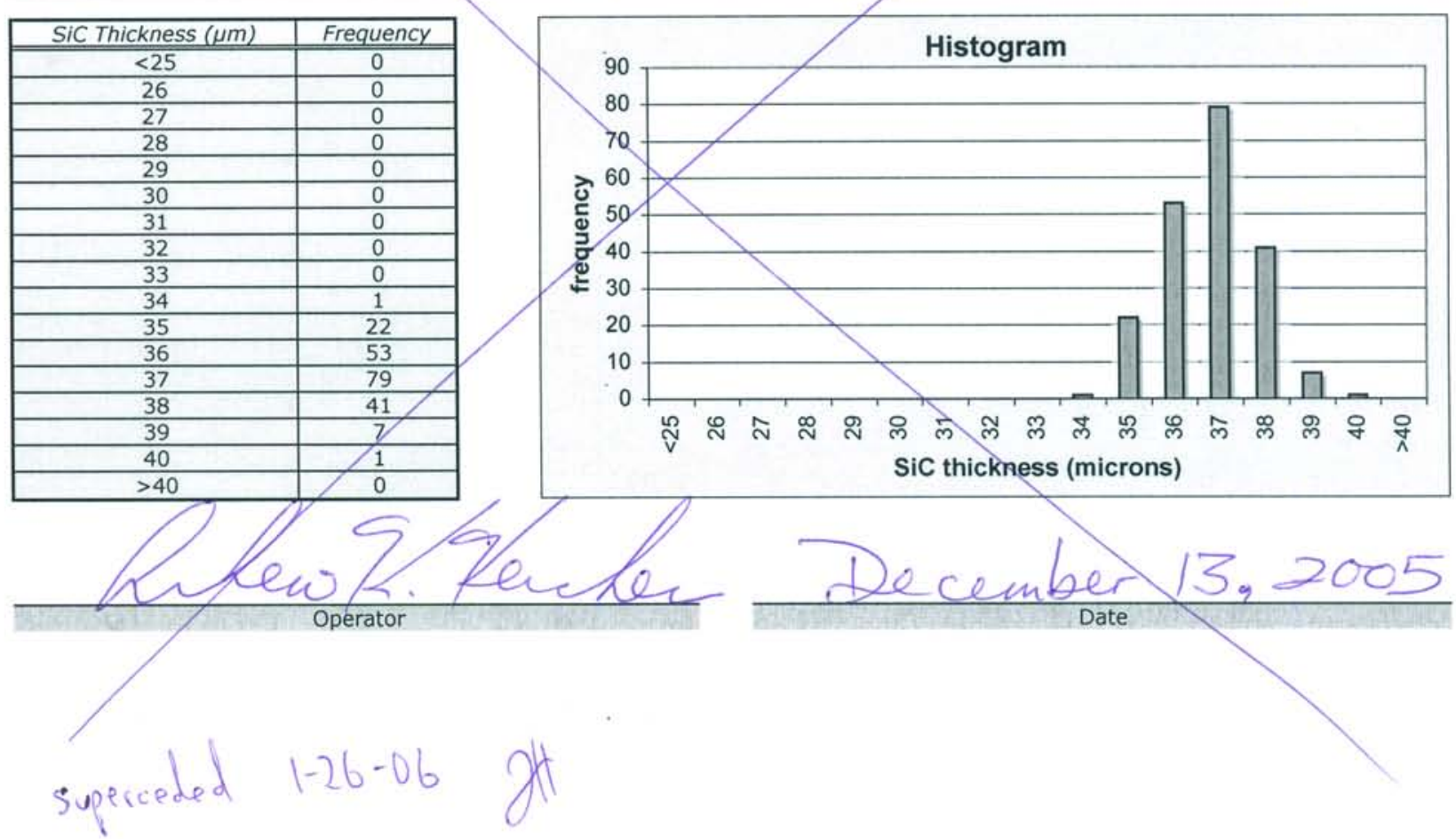




\section{Data Report Form DRF-11D: Measurement of Outer Pyrocarbon Layer Thickness}

\begin{tabular}{|c|c|c|}
\hline- & Procedure: & AGR-CHAR-DAM-11 Rev, 1 \\
\hline & Operator: & Andrew K. Kercher \\
\hline+ & Folder Name: & 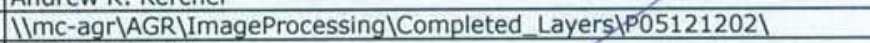 \\
\hline & Sample ID: & LEU01-35T-B01 \\
\hline & Sample Description: & TRISO on BWXT kernel composite 69302 \\
\hline
\end{tabular}

teted_Layers

\begin{tabular}{|r|c|}
\hline Number of outer pyrocarbon layers analyzed: & 204 \\
\hline Mean of the average OPyC thickness of each particle $(\mu \mathrm{m}):$ & 38.0 \\
\hline Standard deviation in the average OPyC thickness of each particle $(\mu \mathrm{m}):$ & 2.4 \\
\hline
\end{tabular}

Distribution of the average OPyC laver thickness (top binned)

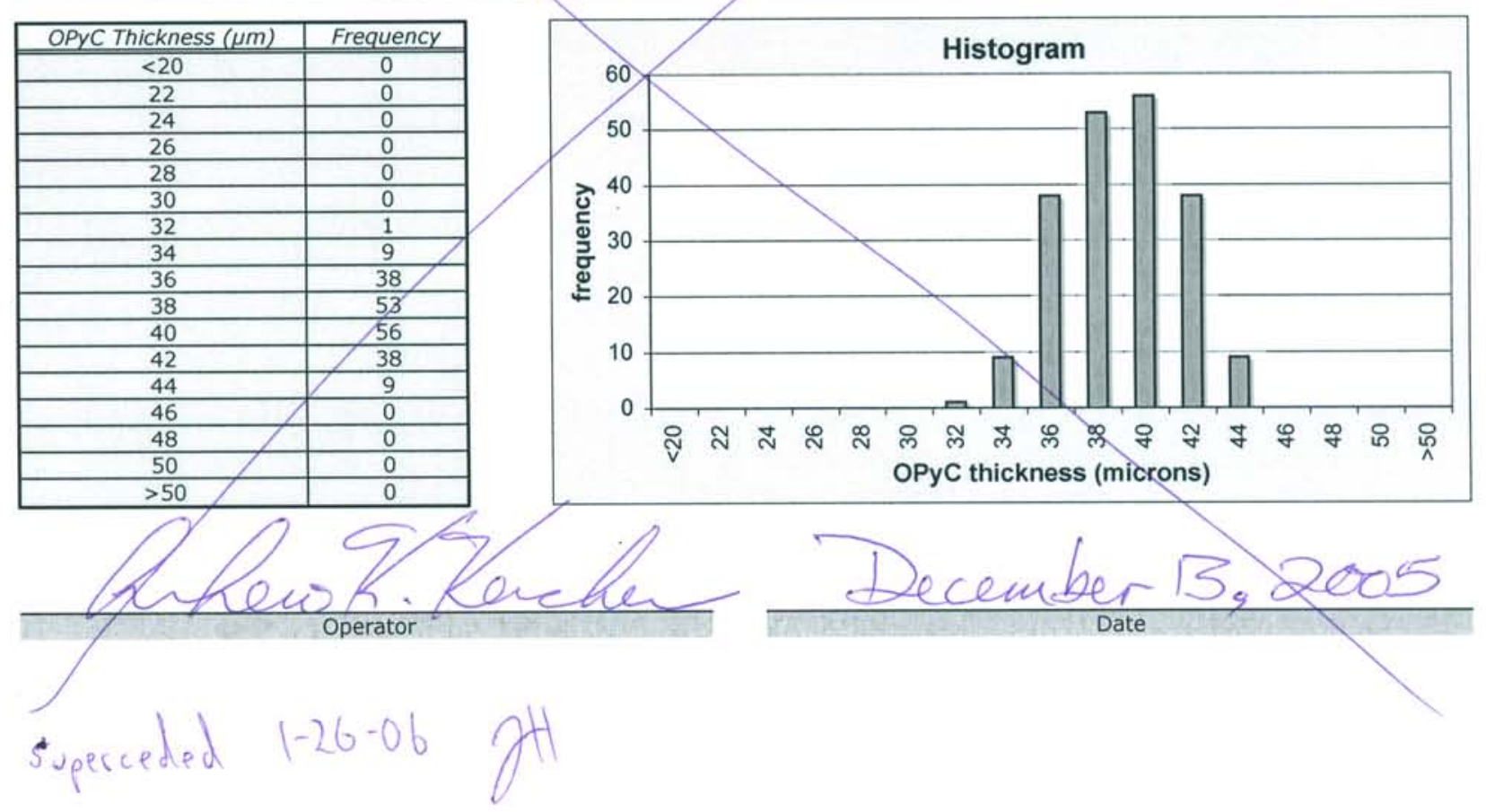


ORNL/TM-2006/019, Rev. 1

Inspection Report Form IRF-03: Coated Particle Batches

Procedure: $\mid$ AGR-CHAR-PIP-03 Rev. 2

Coated particle batch ID: LEU01-27T

Coated particle batch description: TRISO on BWXT kernel composite 69302

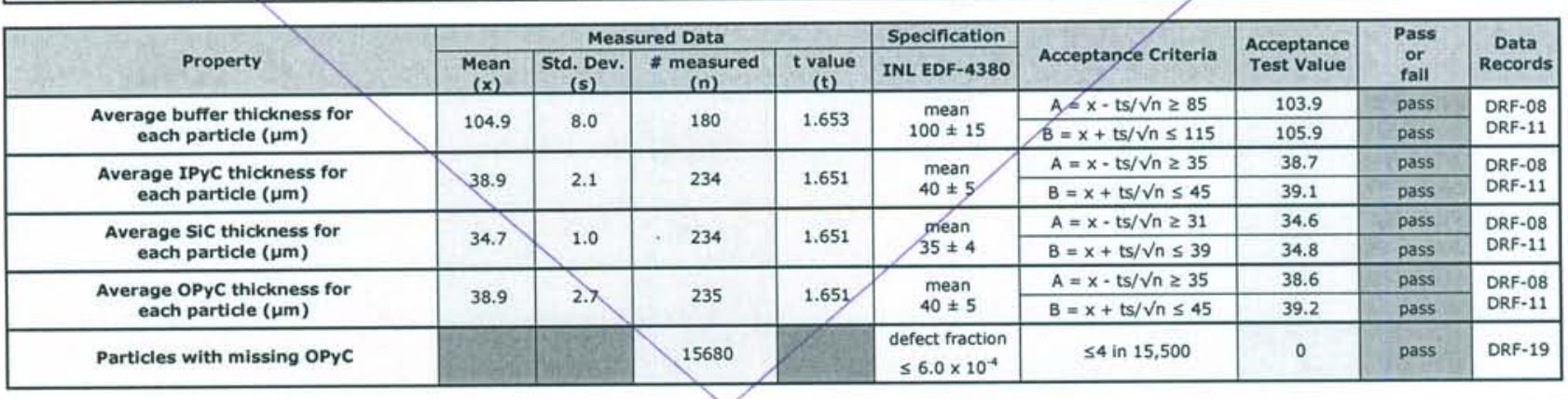

Comments

Goldspot analysis performed on missing OPyC sample for information only. 31 out of 15680 gold spots observed.
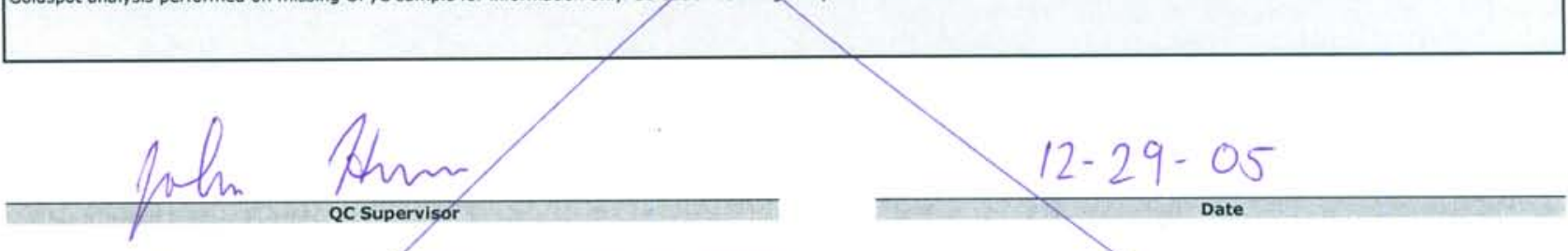

Accept Coated particle batch (Yes or No)

Yes
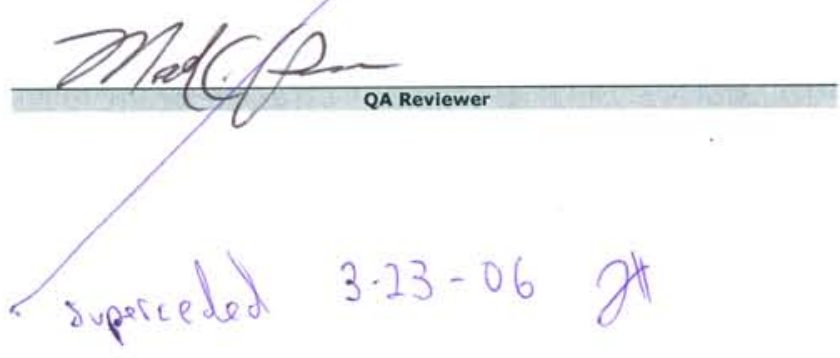
Data Report Form DRF-11A: Measurement of Buffer Layer Thickness

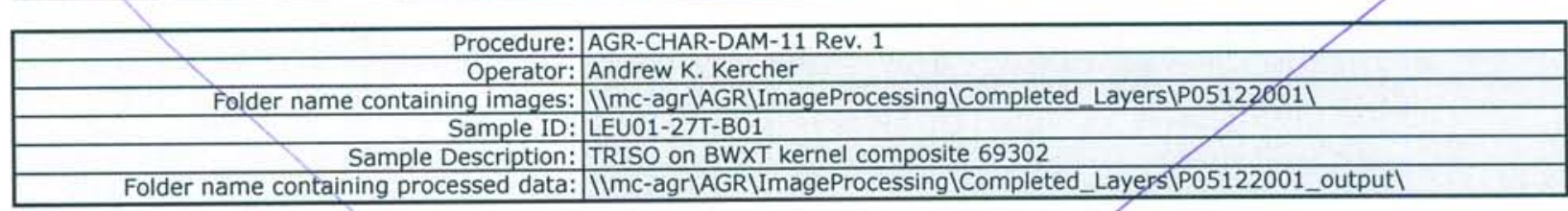

\begin{tabular}{|r|r|}
\hline Number of buffer layers analyzed: & 180 \\
\hline Mean of the average buffer thickness of each particle $(\mu \mathrm{m}):$ & 104.9 \\
\hline Standard deviation in the average buffer thickness of each particle $(\mu \mathrm{m}):$ & 8.0 \\
\hline
\end{tabular}

\section{Distribution of the average buffer layer thickness (top binned)}

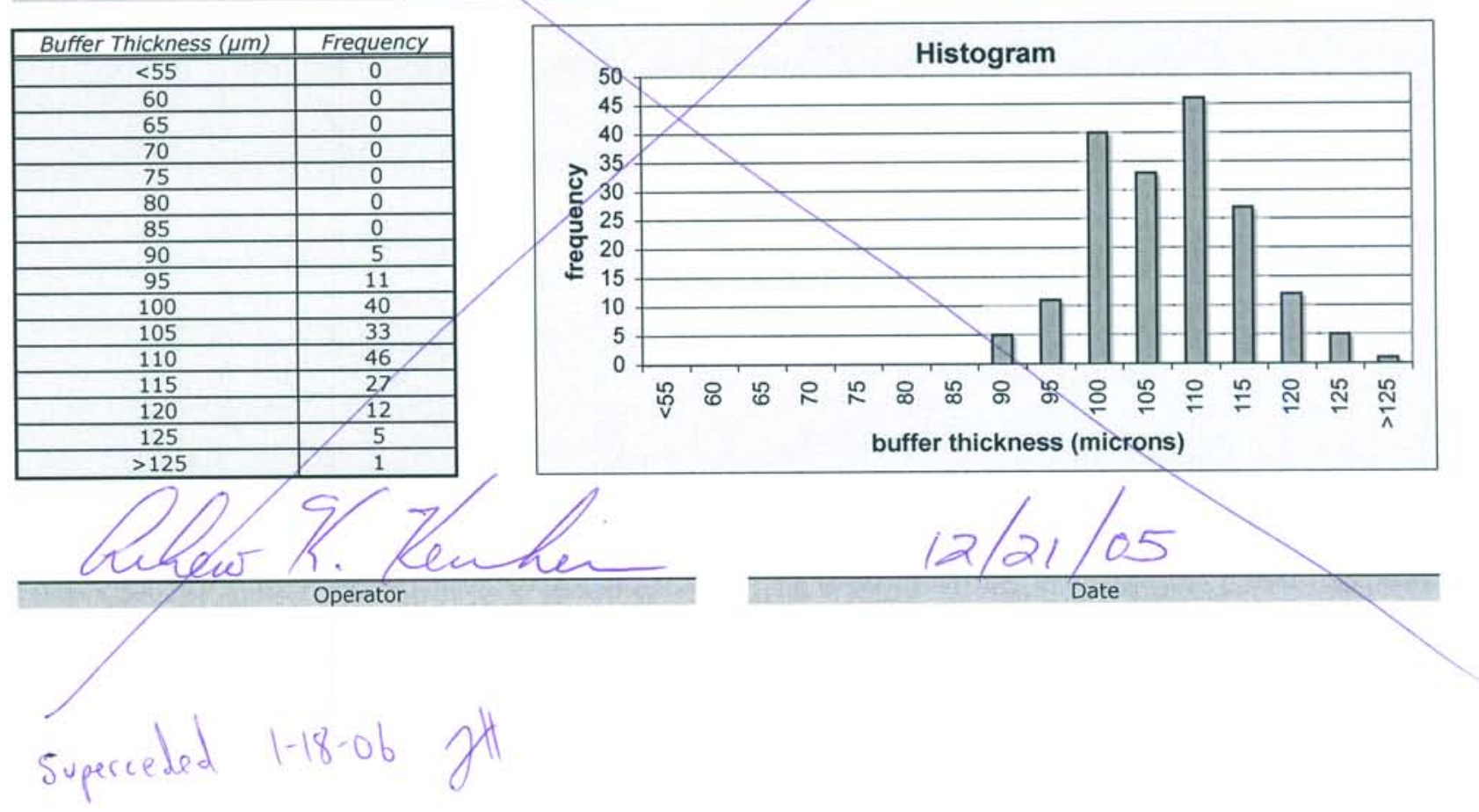


Data Report Form DRF-11B: Measurement of Inner Pyrocarbon Layer Thickness

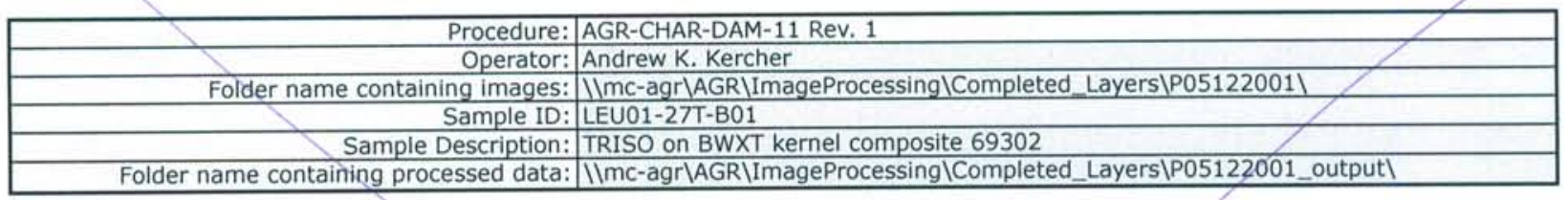

\begin{tabular}{|r|r|r|}
\hline Number of inner pyrocarbon layers analyzed: & Mean of the average IPyC thickness of each particle $(\mu \mathrm{m}):$ & 234 \\
\hline Standard deviation in the average IPyC thickness of each particle $(\mu \mathrm{m}):$ & 38.9 \\
\hline
\end{tabular}

\section{Distribution of the average IPyC layer thickness (top binned)}

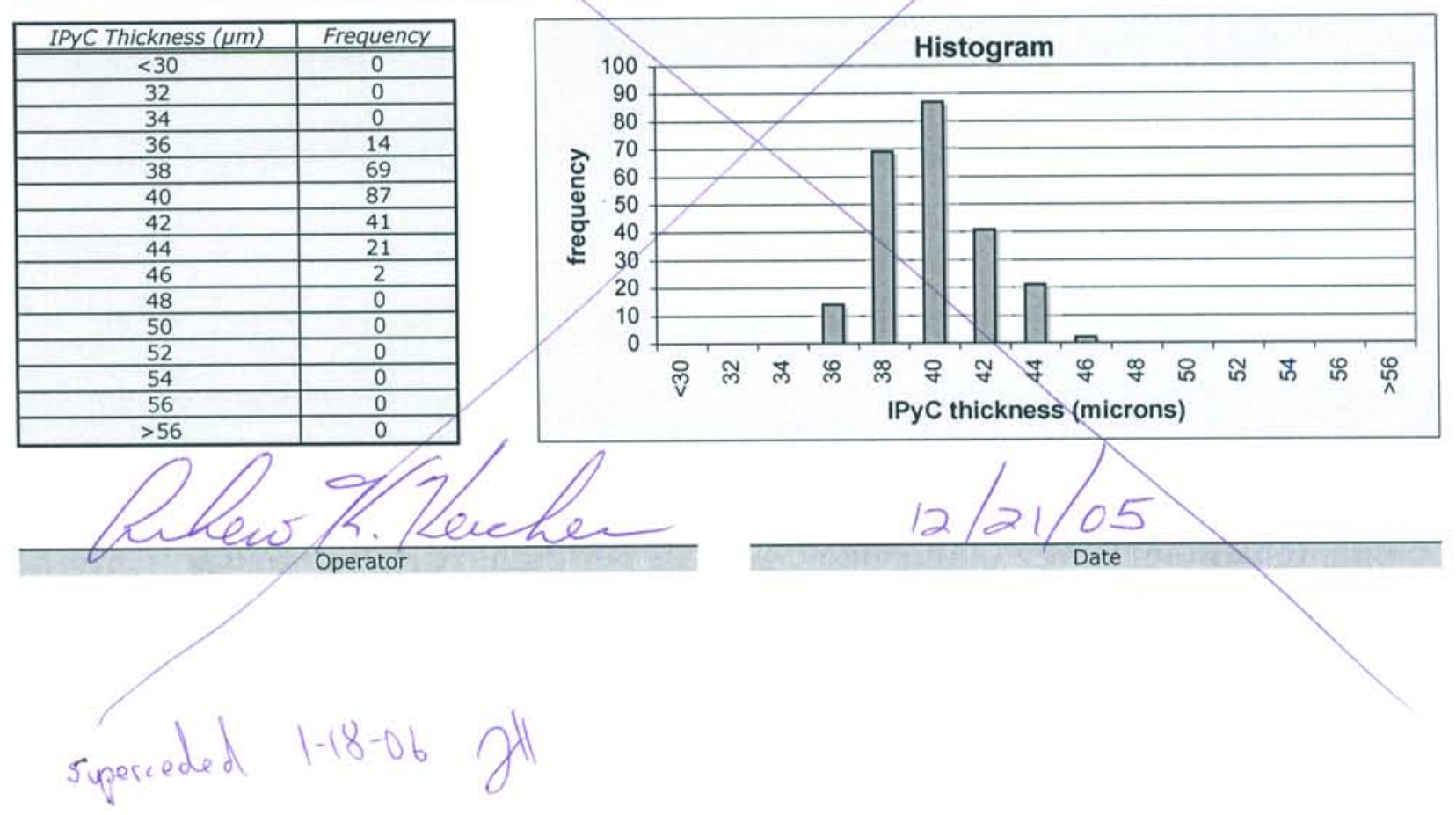


Data Report Form DRF-11C: Measurement of Silicon Carbide Layer Thickness

\begin{tabular}{|c|c|}
\hline Procedure: & AGR-CHAR-DAM-11 Rev, 1 \\
\hline Operator: & Andrew K. Kercher \\
\hline Folder name containing images: & 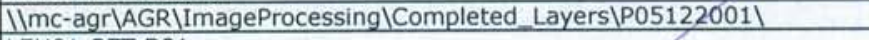 \\
\hline Sample ID: & LEU01-27T-B01 \\
\hline Sample Description: & TRISO on BWXT kernel composite 69302 \\
\hline Folder name containing processed data: & 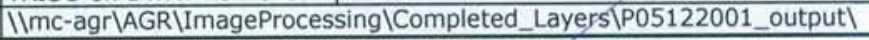 \\
\hline
\end{tabular}

Folder name containing processed data: IImc-agr|AGR|ImageProcessinglCompleted_Layers TP05122001_output

\begin{tabular}{|r|r|}
\hline Namber of silicon carbide layers analyzed: & \\
\hline Mean of the average SiC thickness of each particle $(\mu \mathrm{m}):$ & \\
\hline Standard deviation in the average SiC thickness of each particle $(\mu \mathrm{m}):$ & 334 \\
\hline
\end{tabular}

\section{Distribution of the average SiC layer thickness (top binned)}

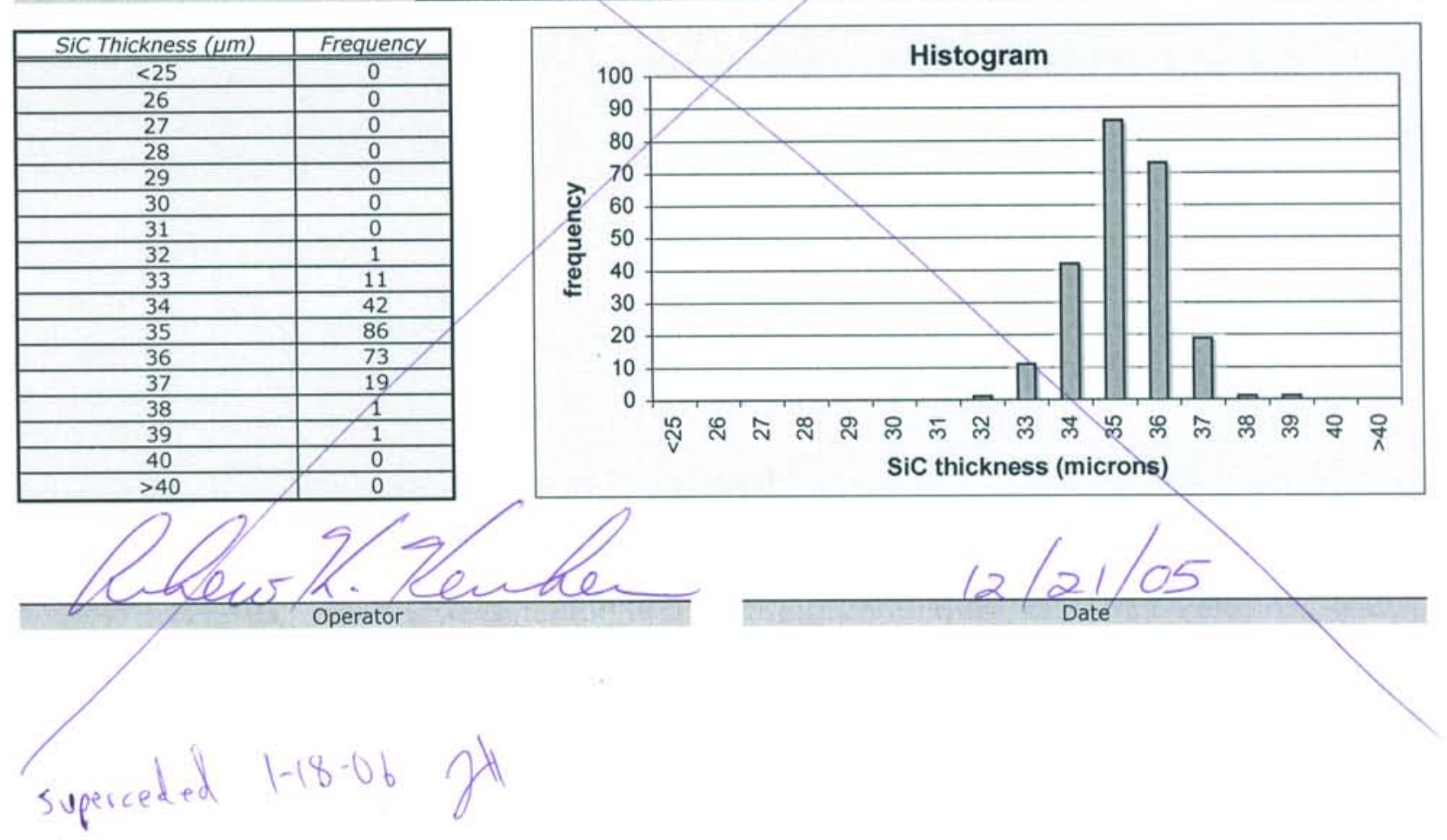




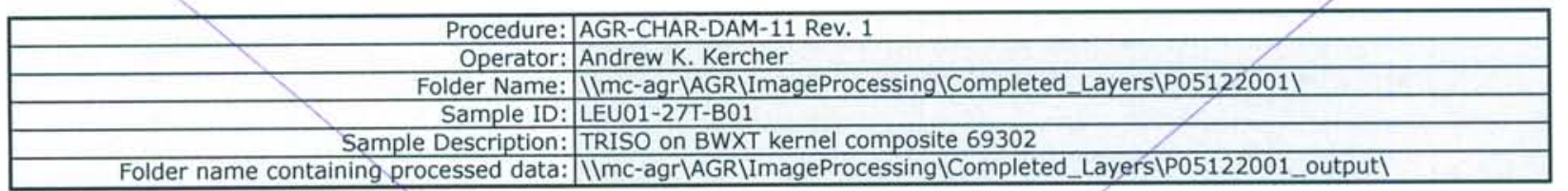

\begin{tabular}{|r|r|r|}
\hline Number of outer pyrocarbon layers analyzed: & 235 \\
\hline Mean of the average OPyC thickness of each particle $(\mu \mathrm{m}):$ & 38.9 \\
\hline Standard deviation in the average OPyC thickness of each particle $(\mu \mathrm{m}):$ & 2.7 \\
\hline
\end{tabular}

\section{Distribution of the average OPyC layer thickness (top binned)}

\begin{tabular}{|c|c|}
\hline OPyC Thickness $(\mu \mathrm{m})$ & Frequency \\
\hline \hline$<20$ & 0 \\
\hline 22 & 0 \\
\hline 24 & 0 \\
\hline 26 & 0 \\
\hline 28 & 0 \\
\hline 30 & 0 \\
\hline 32 & 0 \\
\hline 34 & 7 \\
\hline 36 & 25 \\
\hline 38 & 57 \\
\hline 40 & 60 \\
\hline 42 & 60 \\
\hline 44 & 19 \\
\hline 46 & 5 \\
\hline 48 & 2 \\
\hline 50 & 0 \\
\hline$>50$ & 0 \\
\hline
\end{tabular}
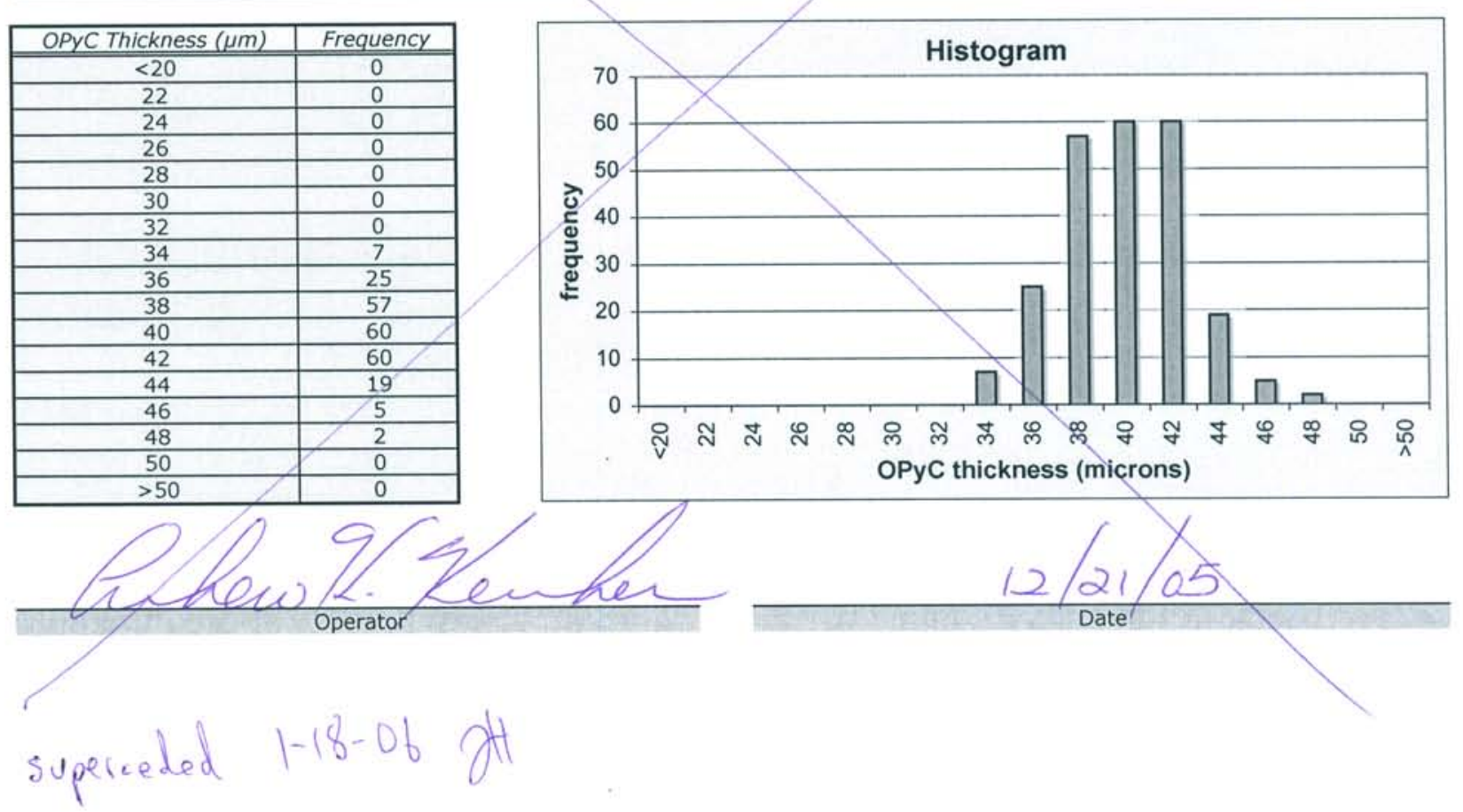
ORNL/TM-2006/019, Rev. 1

Inspection Report Form IRF-03; Coated Particle Batches

Procedure. AGR-CHAR-RIP-03 Rev. 2

Coated particle batch ID: LEU01-45T

Coated particle batch description: TRISO on BWXT kernel composite 69302

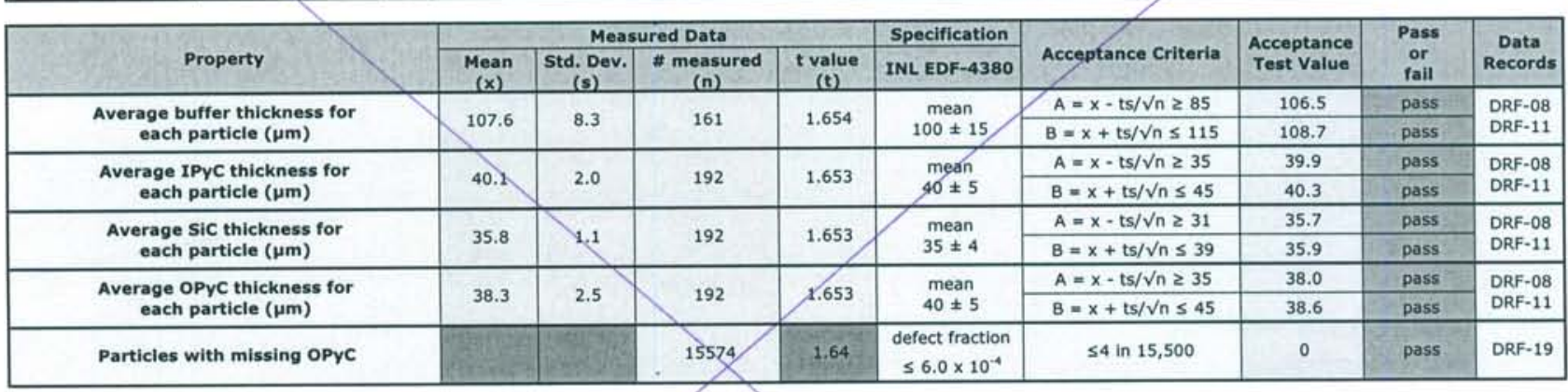

\section{Comments}

Goldspot analysis performed on missing OPyC sample for information only/ 3 out of 15574 gold spots observed.

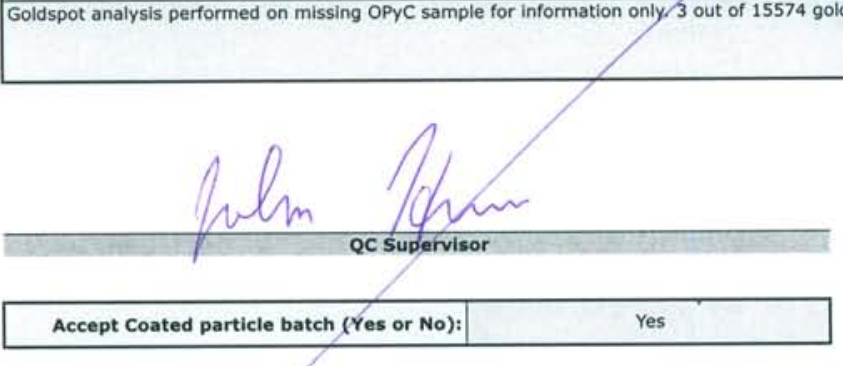

QA Revi

QA Reviewer

cerved

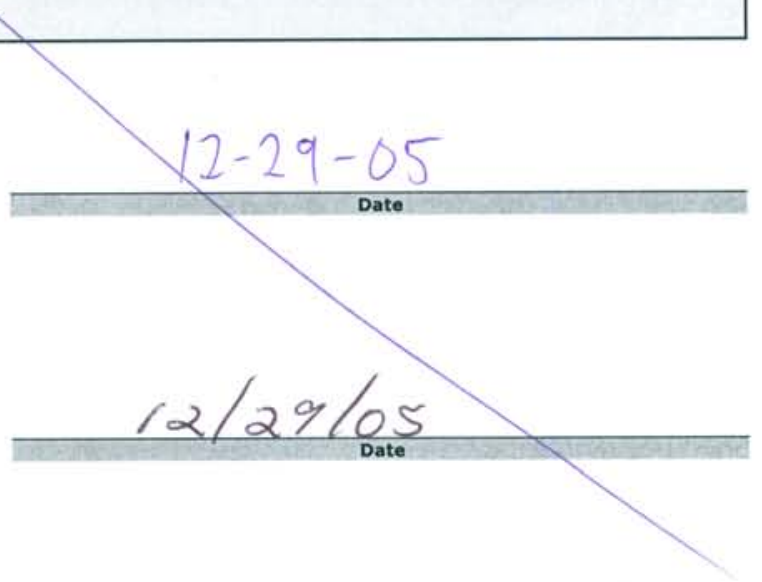

superceded 3.23-06 ott. 


\section{Data Report Form DRF-11A: Measurement of Buffer Layer Thickness}

\begin{tabular}{|c|c|c|}
\hline \multicolumn{3}{|c|}{ Procedure: $\mid$ AGR-CHAR-DAM-11 Rev. 1} \\
\hline Operator: & \\
\hline Folder name containing images: & \multicolumn{2}{|c|}{ IImc-agr|AGR\ImageProcessing|Completed_Layers\P05122201 } \\
\hline Sample ID: & \multicolumn{2}{|c|}{ LEU01-45T-B01 } \\
\hline Sample Description: & \multicolumn{2}{|c|}{ TRISO on BWXT kernel composite 69302} \\
\hline Folder name containing processed data: & \multicolumn{2}{|c|}{ IImc-agr|AGR\ImageProcessing|Completed_Layers\P05122201_output } \\
\hline$>$ & Number of buffer layers analyzed: & 161 \\
\hline Mean of the & average buffer thickness of each particle $(\mu \mathrm{m})$ : & 107.6 \\
\hline Standard deviation in the & average buffer thickness of each particle $(\mu \mathrm{m})$ : & 8.3 \\
\hline
\end{tabular}

\section{Distribution of the average buffer layer thickness (top binned)}

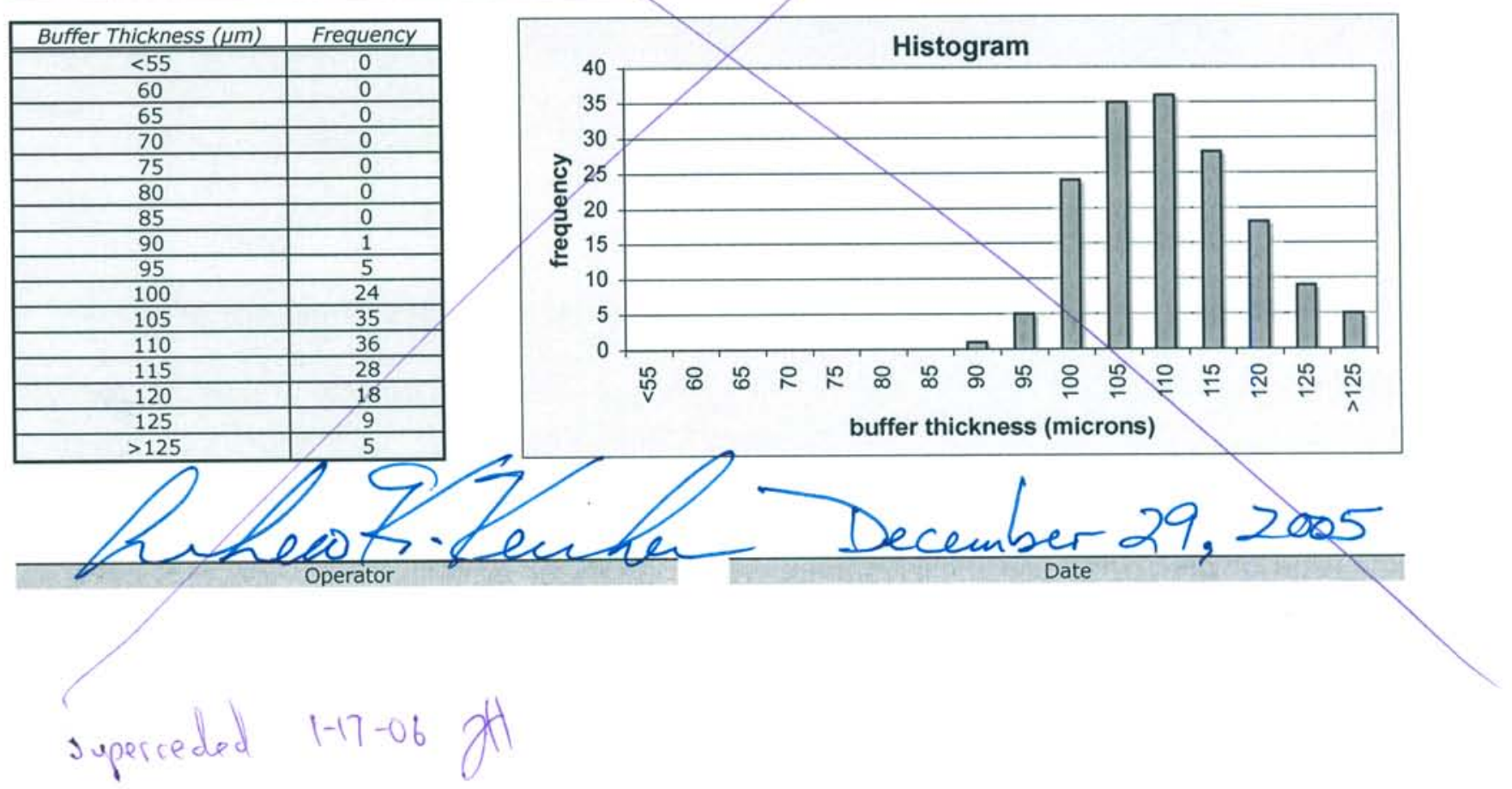




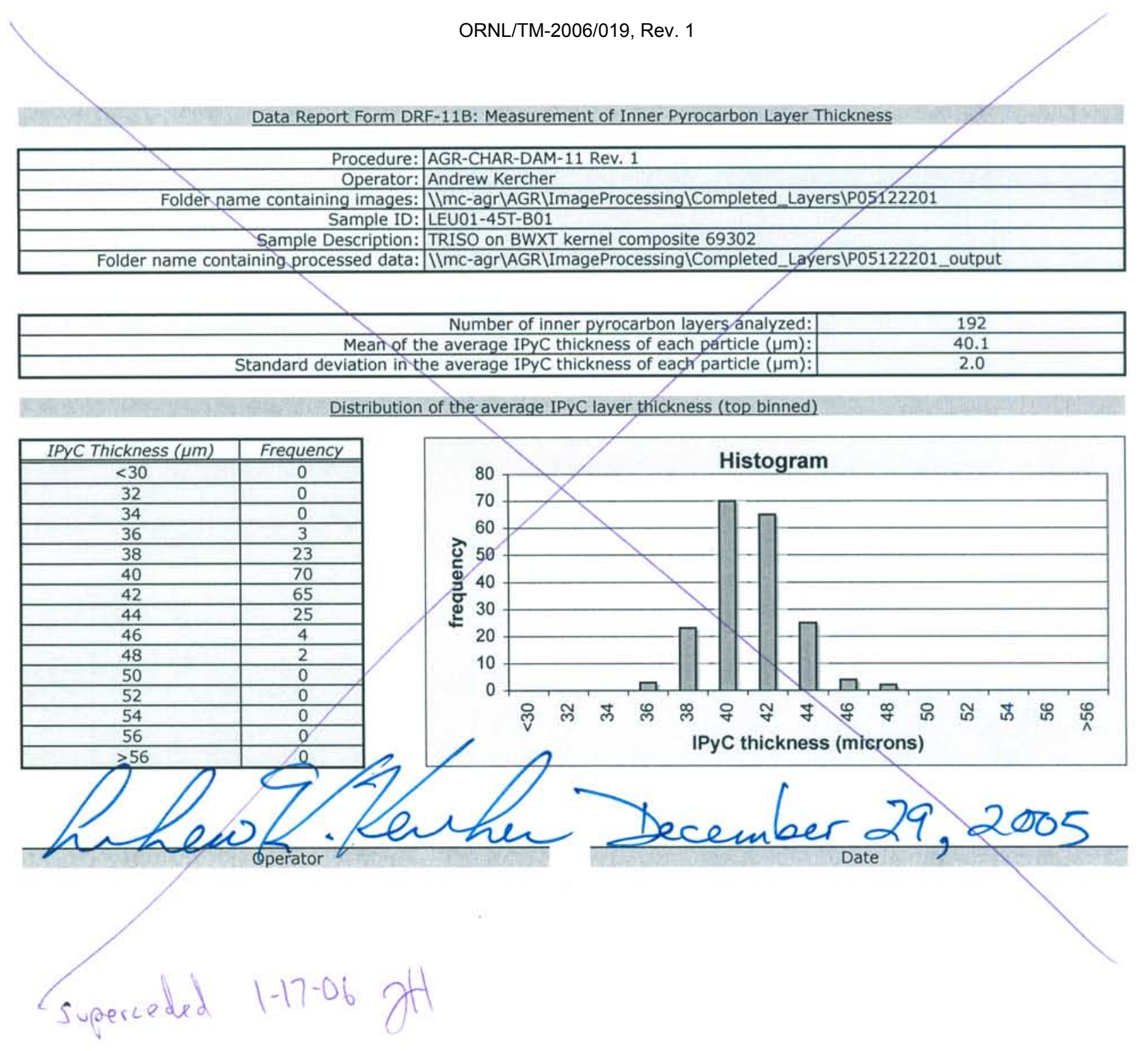

233 


\section{Data Report Form DRF-11C: Measurement of Silicon Carbide Layer Thickness}

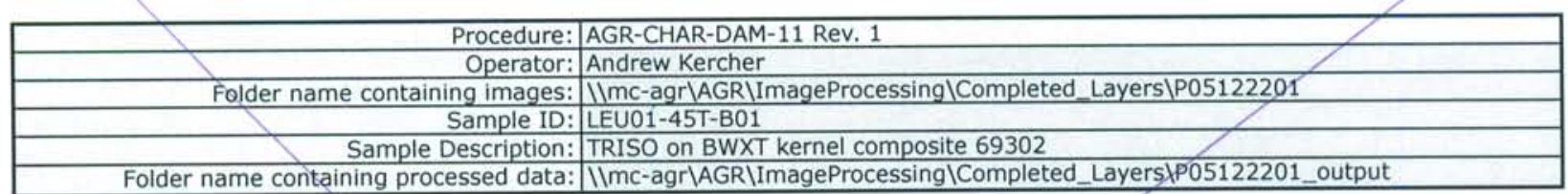

Folder name containing processed data: \Imc-agr\AGR\ImageProcessing\Completed_Layers\P05122201_output

\begin{tabular}{|r|r|}
\hline Number of silicon carbide layers analyzed: & Mean of the average SiC thickness of each particle $(\mu \mathrm{m}):$ \\
\hline Standard deviation in the average SiC thickness of each particle $(\mu \mathrm{m}):$ & 35.8 \\
\hline
\end{tabular}

\section{Distribution of the average SiC layer thickness (top binned)}

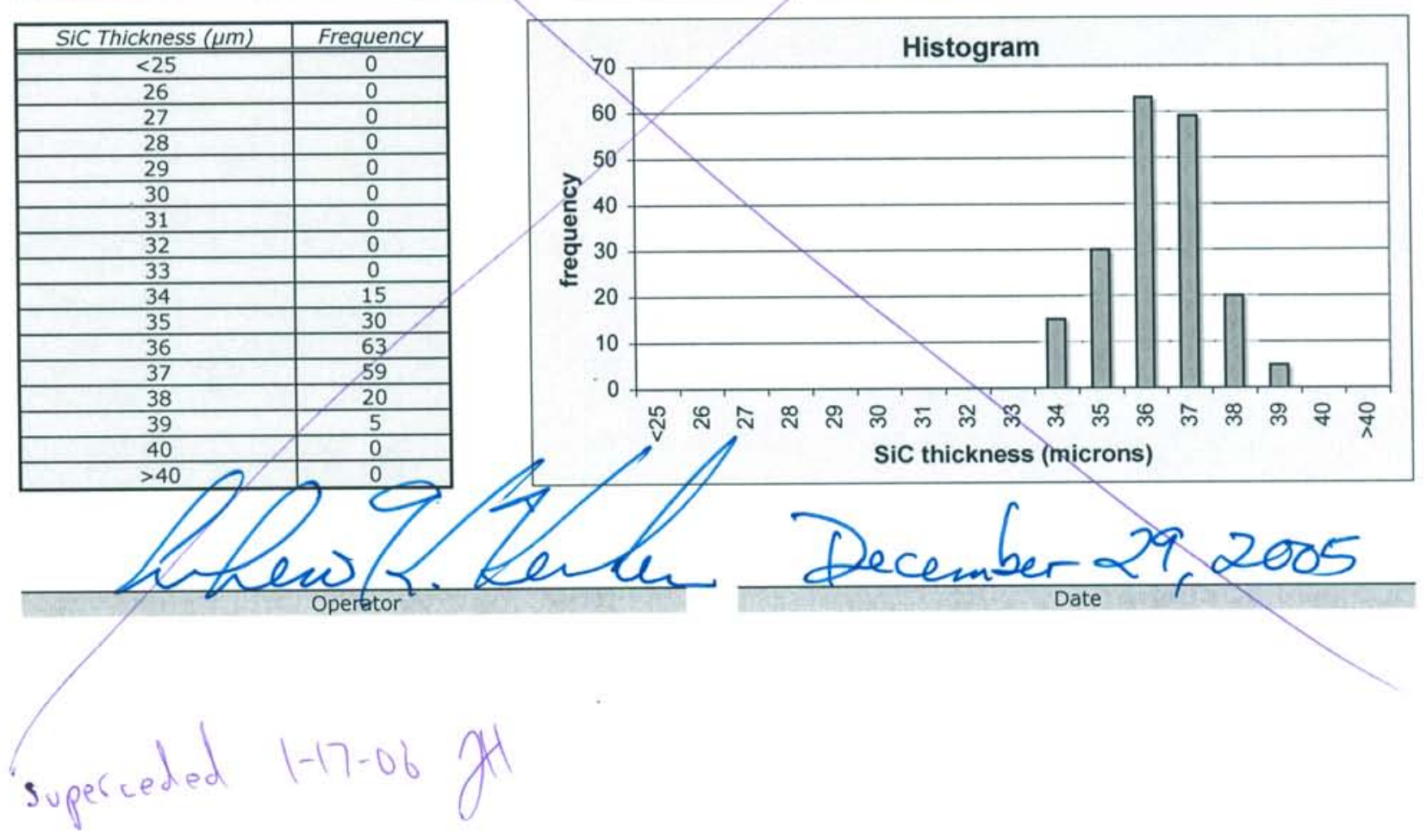


Data Report Form DRF-11D: Measurement of Outer Pyrocarbon Layer Thickness

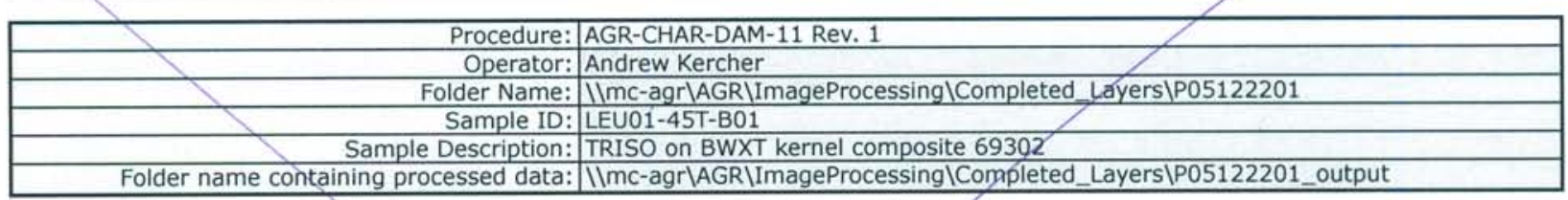

\begin{tabular}{|r|c|}
\hline Number of outer pyrocarbon layers analyzed: & 192 \\
\hline Mean of the average OPyC thickness of each particle $(\mu \mathrm{m}):$ & 38.3 \\
\hline Standard deviation in the average OPyC thickness of each particle $(\mu \mathrm{m}):$ & 2.5 \\
\hline
\end{tabular}

Distribution of the average OPyC layer thickness (top binned)

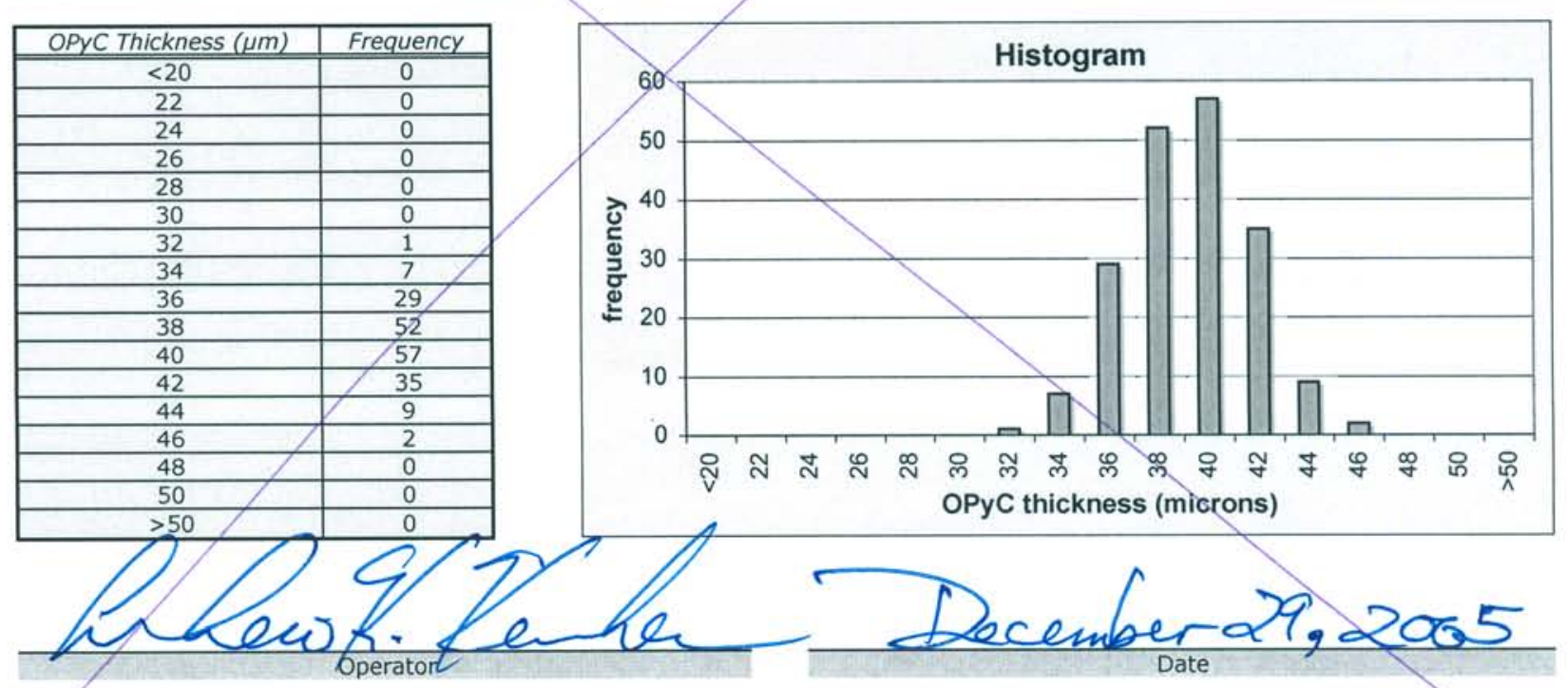

superceded $1-17-06$ 